University of Tennessee Health Science Center UTHSC Digital Commons

$12-2018$

\title{
The Tumor Microenvironment Regulates Retinoblastoma Cell Survival
}

Zachary K. Goldsmith

University of Tennessee Health Science Center

Follow this and additional works at: https://dc.uthsc.edu/dissertations

Part of the Eye Diseases Commons, Medical Cell Biology Commons, Neoplasms Commons, and the Ophthalmology Commons

\section{Recommended Citation}

Goldsmith, Zachary K. (http://orcid.org/0000-0002-7393-733), "The Tumor Microenvironment Regulates Retinoblastoma Cell Survival" (2018). Theses and Dissertations (ETD). Paper 467. http://dx.doi.org/ 10.21007/etd.cghs.2018.0472.

This Dissertation is brought to you for free and open access by the College of Graduate Health Sciences at UTHSC Digital Commons. It has been accepted for inclusion in Theses and Dissertations (ETD) by an authorized administrator of UTHSC Digital Commons. For more information, please contact jwelch30@uthsc.edu. 


\title{
The Tumor Microenvironment Regulates Retinoblastoma Cell Survival
}

\begin{abstract}
Retinoblastoma $(\mathrm{Rb})$ is the most common intraocular malignancy in children comprising $4 \%$ of all pediatric tumors. Early intervention increases survival rates up to $95 \%$ in developed countries; being survival rates associated to socio-economic status. Despite the high survival rates in developed countries, preservation of the eye and vision are continuing challenges in the management of Rb. Vitreous seeds constitute the greatest challenge in treatment of $\mathrm{Rb}$. The unique location of these seeds within the vitreous makes them difficult to treat. Viable seeds showed reduced proliferative capacity and metabolism. making the majority refractory to current chemotherapeutics. This prompted the development of new delivery routes for chemotherapeutics, such as intra-ophthalmic artery, intravitreal, and subconjunctival administration. Still ocular salvage rates have not exceeded $70 \%$ in over two decades. Novel, safer therapies are required but there is a fundamental lack of knowledge about the biology of the tumor for the development of targeted therapies; the vitreous, as it surrounds the seeds; and the interactions between the vitreous and the seeds.
\end{abstract}

Despite the current understanding of how cancer is a multifaceted disease full of complex cellular and protein interactions (commonly referred to as the tumor microenvironment, TME), the immunology of the vitreous microenvironment and the role it plays in the sustainment of seeds in $\mathrm{Rb}$ disease is poorly understood. In this study, we begin investigating Rb tumor cells and how they alter the TME by examination of matrix metalloproteinases, a family of enzymes involved in degradation of the extracellular matrix and tissue remodeling heavily implicated in tumor migration and survival. We identified two gelatinases, MMP-2 and MMP-9, to be expressed in Rb cell lines and demonstrated by pharmacological inhibition and genetic knockdown, a role for these gelatinases in Rb cell migration, invasion, and survival. Additionally, we demonstrated how secretion of VEGF, involved in angiogenesis, and TGF $\beta$, involved in metastasis, were altered by MMP-2 and MMP-9 pharmacological inhibition.

As MMPs are pivotal for the tumor and extracellular matrix interactions within the TME leading to tissue invasion, we then transitioned to the vitreous, investigating the vitreous as a TME and how it sustains $\mathrm{Rb}$. Multiple proteins are found within the soluble phase of the vitreous that are associated with ocular pathological processes, including diabetes retinopathy and proliferative vitreoretinopathy. Therefore, we compared the presence of a small cohort of proteins associated to ocular pathologies, to healthy vitreous and to $\mathrm{Rb}$ patients' vitreous samples, identifying high expression of Platelet-Derived Growth Factor Receptor $\beta$ (PDGFR $\beta$ ) and its ligand PDGFBB in the Rb samples. Additional studies of ex vivo healthy human vitreous, murine $\mathrm{Rb}$ xenografts, and patient-derived $\mathrm{Rb}$ xenograft tissues, measured high activity of the PDGF-PDGFR $\beta$ signaling pathway in diseased, but not healthy tissue. We focused the next part of this work on the role PDGFR $\beta$ plays in vitreous seeds. To investigate this pathway in depth we used established Rb cell lines, namely Y79-the metastatic and aggressive model, and Weri-1 Rb, the nonmetastatic model. Our work demonstrated PDGFR $\beta$ was overexpressed in $Y 79$ cells, the metastatic model of $\mathrm{Rb}$, in vitro. To provide mechanistic insight, we utilized the tyrosine kinase inhibitor imatinib mesylate (IM), and demonstrated PDGF-PDGFR $\beta$ signaling pathway regulates $\mathrm{Rb}$ cell proliferation, invasion, and survival. We found increased PDGF-PDGFR $\beta$ signaling resulted in higher activity of the p53-inactivator, MDM2, as well as the pro-inflammatory NF $\beta B$ pathway, both of which are involved in tumor survival. As there is a lack of models for vitreous seeding, we utilized magnetic nanoparticles to generate the first in vitro vitreous seed model and demonstrated similar features between an ex vivo seed and one of our in vitro magnet-generated 3D tumor spheroids, validating our system. Using this novel technology, we recapitulated our in vitro 2D work and demonstrated that inhibition of the PDGF-PDGFR $\beta$ signaling pathway results in decreased spheroid size. PDGFRB gene knockdown by siRNA confirmed the results with the pharmacological agent showing these results were PDGF-PDGFR $\beta$ signaling-specific. 
Next, we tested the effects of IM, as a potential Rb therapy, in retinal endothelial cells $\mathrm{h}$ (RECs) as retinal endothelial cell-associated toxicities are one of the challenges with conventional chemotherapies in $\mathrm{Rb}$. We measured the capacity of hREC to proliferate and for tube formation in the presence of the therapy. Our results demonstrated neither proliferation nor tube formation of hRECs changed when exposed to IM. Upon further examination, we demonstrated the absence of PDGFRB mRNA expression in hRECs. Taken together these results illustrate the potential use of anti-PDGFR $\beta$ therapy as a targeted therapy in Rb.

The last section of the study investigated the expression and function of members of the ATP-binding cassette (ABC) transporters as well as the multifunctional glycoprotein CD44 in Rb, as one of the properties of vitreous seeds is their resistance to chemotherapy. We discovered heterogeneous expression of multiple $A B C$ transporters in vitro using $Y 79 \mathrm{Rb}$ cell line and ex vivo using $\mathrm{Rb}$ vitreous samples. In addition to expression, the transporters efflux activity was determined. CD44 mRNA, whose protein is considered a promoter of chemoresistance, was highly expressed in naïve vitreous from $\mathrm{Rb}$ patients. These results illustrate that chemoresistance is not induced by treatment and is, instead, an innate feature of vitreous seeds. Flow cytometry analyses established (1) a high correlation between active, or phosphorylated PDGFR $(\mathrm{p}-\mathrm{PDGFR} \beta$ ) and CD44; and (2) inhibition of p-PDGFR $\beta$ resulted in decreased CD44, as well.

Collectively, this study demonstrated the Rb TME plays a crucial role in Rb tumor properties. MMP-2 and MMP-9 are highly expressed in Rb and allow degradation of the ECM in tissue, increasing migration and invasion of $\mathrm{Rb}$. Additional work focused on vitreous seeds and identified increasing signaling activity of the PDGF-PDGFR $\beta$ signaling pathway in multiple Rb models, including in vitro cell lines, ex vivo Rb patient vitreous samples and tissue, as well as in a murine xenograft system. Next, we identified this pathway as a potential target, as pharmacological and genomic inhibition of the PDGF-PDGFR $\beta$ signaling pathway by IM and PDGFRB siRNA resulted in decreased proliferation, invasion, resistance, and survival. These cellular functions appear to be mediated by the downstream targets MDM2 and NFßB. Our initial studies demonstrated a lack of drug-associated toxicity on hRECs with IM. This work is a further step in our quest for targeting vitreous seeding.

\section{Document Type}

Dissertation

\section{Degree Name}

Doctor of Philosophy (PhD)

\section{Program}

Biomedical Sciences

\section{Research Advisor}

Monica M. Jablonski, Ph.D.

\section{Keywords}

Ocular immunology, Ocular oncology, Ophthalmology, Retinoblastoma, Tumor biology, Tumor microenvironment

\section{Subject Categories}

Diseases | Eye Diseases | Medical Cell Biology | Medical Sciences | Medical Specialties | Medicine and Health Sciences | Neoplasms | Ophthalmology 


\title{
The Tumor Microenvironment Regulates Retinoblastoma Cell Survival
}

\author{
A Dissertation \\ Presented for \\ The Graduate Studies Council \\ The University of Tennessee \\ Health Science Center
}

\author{
In Partial Fulfillment \\ Of the Requirements for the Degree \\ Doctor of Philosophy \\ From The University of Tennessee
}

By

Zachary K. Goldsmith

December 2018 
Chapter 2 (C) 2017 by BioMed Central.

Chapter 3 (c) 2018 by The Association for Research in Vision and Ophthalmology. All other material (C) 2018 by Zachary Keith Goldsmith.

All rights reserved. 


\section{DEDICATION}

I dedicate this dissertation to my parents: my father, Mr. Noel Keith Goldsmith, who first instilled curiosity and a love of science in me, and my mother, Mrs. Pamela Jean Goldsmith, who taught me by example the value of dedication and hard work; she always told me I would "move mountains." Their unconditional love, support, and

encouragement made this dissertation possible. This achievement is as much theirs as is it is mine. 


\section{ACKNOWLEDGEMENTS}

I would like to extend my deepest gratitude to my mentor, Dr. Vanessa M. Morales-Tirado for her support and trust. Over the past years, Dr. Morales provided an exciting, nurturing environment that allowed me to develop as a scientist and accomplish the work described herein. I always received unflinching encouragement, guidance, and support, and for that I am eternally grateful. I learned so much from her, including science, but also so much more. Thank you for being the best mentor and leader.

I also want to acknowledge Dr. Matthew Wilson for sharing his expertise with me. This work would have never been initiated were it not for his motivation. Dr. Wilson inspired me to be the best I could be, and this work reflects that influence. Every email, every meeting, and every discussion provided indispensable insight and I would not be here without him.

I am grateful to my committee members, Drs. Monica Jablonski, Lawrence Pfeffer, and Anton Reiner for their valuable input and suggestions in guiding me while conducting my doctoral research. Their commitment to my development was irreplaceable.

I want to thank all members of the ocular oncology team for the vital role they played in my research career. Specifically, I would like to thank Mr. Andrew Irvine, William Coppess, Samuel Barsh, Kelley Yuan, and Nikolas Hopkins as well as Dr. Benjamin King for helping me get more from my research experience. The time spent with my coworkers was productive as well as enjoyable. These people not only became my colleagues, but also my friends.

I am grateful for the Departments of Ophthalmology at Hamilton Eye Institute as well as the UTHSC Neuroscience Institute for providing me a place to call my home the last few years. It has been a pleasure working with all of you. I am also thankful for Fight for Sight and St. Baldrick's Foundation for their support of this work through fellowship awards.

I want to acknowledge my previous undergraduate mentors, specifically Drs. John Boughter and Masaaki Tamura, for their time and effort. Without them, I would have never pursued research as a career.

I need to thank my closest friends: Kevin Hope, Matthew Kirchner, Lee Pribyl, and Samuel "Stu" McAfee. Together, we shared the highs and lows of research. Thank you for all of the laughs. 


\begin{abstract}
Retinoblastoma $(\mathrm{Rb})$ is the most common intraocular malignancy in children comprising $4 \%$ of all pediatric tumors. Early intervention increases survival rates up to $95 \%$ in developed countries; being survival rates associated to socio-economic status. Despite the high survival rates in developed countries, preservation of the eye and vision are continuing challenges in the management of $\mathrm{Rb}$. Vitreous seeds constitute the greatest challenge in treatment of $\mathrm{Rb}$. The unique location of these seeds within the vitreous makes them difficult to treat. Viable seeds showed reduced proliferative capacity and metabolism. making the majority refractory to current chemotherapeutics. This prompted the development of new delivery routes for chemotherapeutics, such as intra-ophthalmic artery, intravitreal, and subconjunctival administration. Still ocular salvage rates have not exceeded $70 \%$ in over two decades. Novel, safer therapies are required but there is a fundamental lack of knowledge about the biology of the tumor for the development of targeted therapies; the vitreous, as it surrounds the seeds; and the interactions between the vitreous and the seeds.

Despite the current understanding of how cancer is a multifaceted disease full of complex cellular and protein interactions (commonly referred to as the tumor microenvironment, TME), the immunology of the vitreous microenvironment and the role it plays in the sustainment of seeds in Rb disease is poorly understood. In this study, we begin investigating $\mathrm{Rb}$ tumor cells and how they alter the TME by examination of matrix metalloproteinases, a family of enzymes involved in degradation of the extracellular matrix and tissue remodeling heavily implicated in tumor migration and survival. We identified two gelatinases, MMP-2 and MMP-9, to be expressed in Rb cell lines and demonstrated by pharmacological inhibition and genetic knockdown, a role for these gelatinases in $\mathrm{Rb}$ cell migration, invasion, and survival. Additionally, we demonstrated how secretion of VEGF, involved in angiogenesis, and TGF $\beta$, involved in metastasis, were altered by MMP-2 and MMP-9 pharmacological inhibition.
\end{abstract}

As MMPs are pivotal for the tumor and extracellular matrix interactions within the TME leading to tissue invasion, we then transitioned to the vitreous, investigating the vitreous as a TME and how it sustains Rb. Multiple proteins are found within the soluble phase of the vitreous that are associated with ocular pathological processes, including diabetes retinopathy and proliferative vitreoretinopathy. Therefore, we compared the presence of a small cohort of proteins associated to ocular pathologies, to healthy vitreous and to Rb patients' vitreous samples, identifying high expression of Platelet-Derived Growth Factor Receptor $\beta$ (PDGFR $\beta$ ) and its ligand PDGFBB in the Rb samples. Additional studies of ex vivo healthy human vitreous, murine Rb xenografts, and patientderived $\mathrm{Rb}$ xenograft tissues, measured high activity of the PDGF-PDGFR $\beta$ signaling pathway in diseased, but not healthy tissue. We focused the next part of this work on the role PDGFR $\beta$ plays in vitreous seeds. To investigate this pathway in depth we used established Rb cell lines, namely Y79-the metastatic and aggressive model, and Weri-1 $\mathrm{Rb}$, the non-metastatic model. Our work demonstrated PDGFR $\beta$ was overexpressed in Y79 cells, the metastatic model of Rb, in vitro. To provide mechanistic insight, we 
utilized the tyrosine kinase inhibitor imatinib mesylate (IM), and demonstrated PDGFPDGFR $\beta$ signaling pathway regulates $\mathrm{Rb}$ cell proliferation, invasion, and survival. We found increased PDGF-PDGFR $\beta$ signaling resulted in higher activity of the p53inactivator, MDM2, as well as the pro-inflammatory NFKB pathway, both of which are involved in tumor survival. As there is a lack of models for vitreous seeding, we utilized magnetic nanoparticles to generate the first in vitro vitreous seed model and demonstrated similar features between an ex vivo seed and one of our in vitro magnet-generated 3D tumor spheroids, validating our system. Using this novel technology, we recapitulated our in vitro $2 \mathrm{D}$ work and demonstrated that inhibition of the PDGF-PDGFR $\beta$ signaling pathway results in decreased spheroid size. PDGFRB gene knockdown by siRNA confirmed the results with the pharmacological agent showing these results were PDGFPDGFR $\beta$ signaling-specific.

Next, we tested the effects of IM, as a potential Rb therapy, in retinal endothelial cells $\mathrm{h}$ (RECs) as retinal endothelial cell-associated toxicities are one of the challenges with conventional chemotherapies in $\mathrm{Rb}$. We measured the capacity of hREC to proliferate and for tube formation in the presence of the therapy. Our results demonstrated neither proliferation nor tube formation of hRECs changed when exposed to IM. Upon further examination, we demonstrated the absence of PDGFRB mRNA expression in hRECs. Taken together these results illustrate the potential use of antiPDGFR $\beta$ therapy as a targeted therapy in $\mathrm{Rb}$.

The last section of the study investigated the expression and function of members of the ATP-binding cassette (ABC) transporters as well as the multifunctional glycoprotein $\mathrm{CD} 44$ in $\mathrm{Rb}$, as one of the properties of vitreous seeds is their resistance to chemotherapy. We discovered heterogeneous expression of multiple $\mathrm{ABC}$ transporters in vitro using $\mathrm{Y} 79 \mathrm{Rb}$ cell line and ex vivo using $\mathrm{Rb}$ vitreous samples. In addition to expression, the transporters efflux activity was determined. CD44 mRNA, whose protein is considered a promoter of chemoresistance, was highly expressed in naïve vitreous from $\mathrm{Rb}$ patients. These results illustrate that chemoresistance is not induced by treatment and is, instead, an innate feature of vitreous seeds. Flow cytometry analyses established (1) a high correlation between active, or phosphorylated PDGFR $\beta$ (p-PDGFR $\beta$ ) and CD44; and (2) inhibition of p-PDGFR $\beta$ resulted in decreased CD44, as well.

Collectively, this study demonstrated the Rb TME plays a crucial role in $\mathrm{Rb}$ tumor properties. MMP-2 and MMP-9 are highly expressed in Rb and allow degradation of the ECM in tissue, increasing migration and invasion of $\mathrm{Rb}$. Additional work focused on vitreous seeds and identified increasing signaling activity of the PDGFPDGFR $\beta$ signaling pathway in multiple $\mathrm{Rb}$ models, including in vitro cell lines, ex vivo $\mathrm{Rb}$ patient vitreous samples and tissue, as well as in a murine xenograft system. Next, we identified this pathway as a potential target, as pharmacological and genomic inhibition of the PDGF-PDGFR $\beta$ signaling pathway by IM and PDGFRB siRNA resulted in decreased proliferation, invasion, resistance, and survival. These cellular functions appear to be mediated by the downstream targets MDM2 and NFאB. Our initial studies demonstrated a lack of drug-associated toxicity on hRECs with IM. This work is a further step in our quest for targeting vitreous seeding. 


\section{TABLE OF CONTENTS}

CHAPTER 1. INTRODUCTION AND BACKGROUND ...........................................1

History of Retinoblastoma Disease ..........................................................................

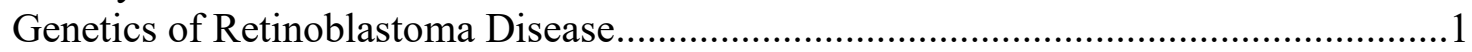

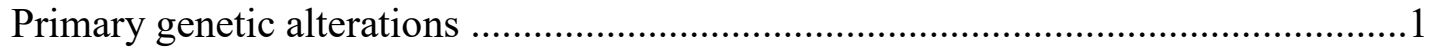

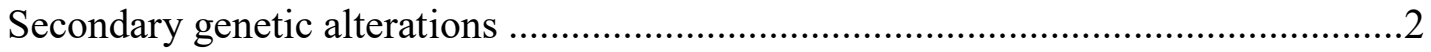

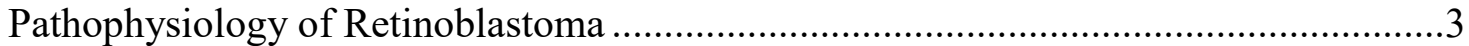

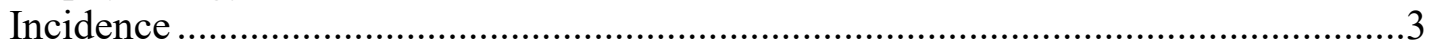

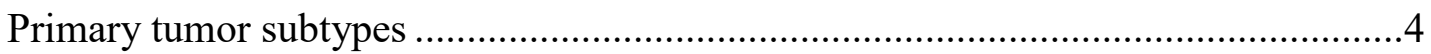

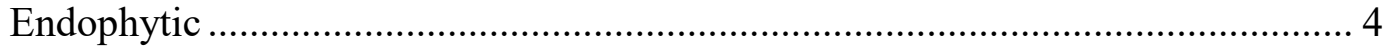

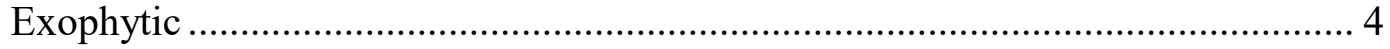

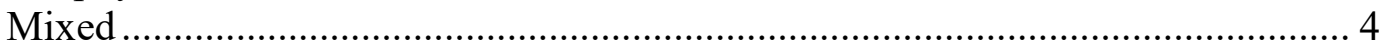

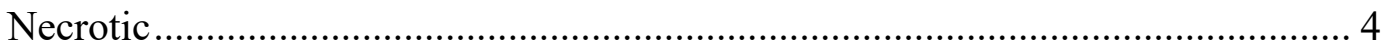

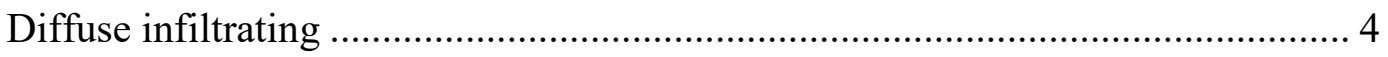

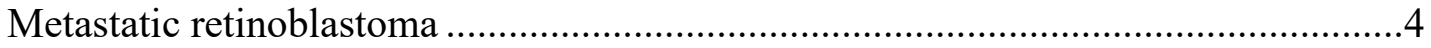

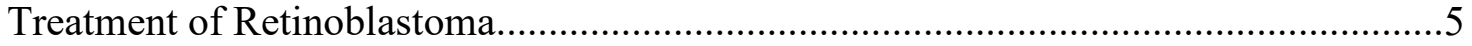

Cryotherapy and thermotherapy.....................................................................5

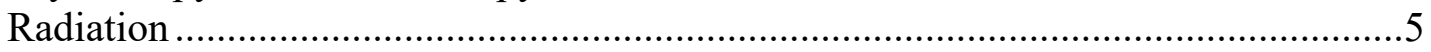

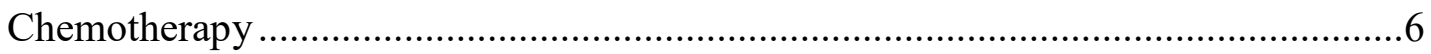

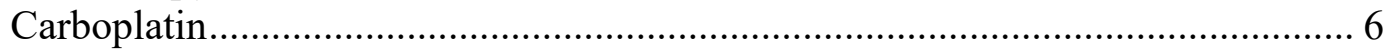

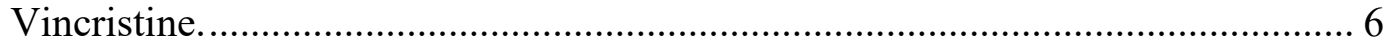

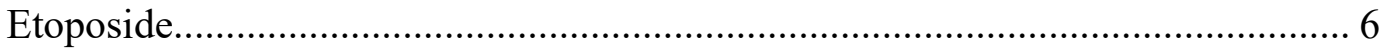

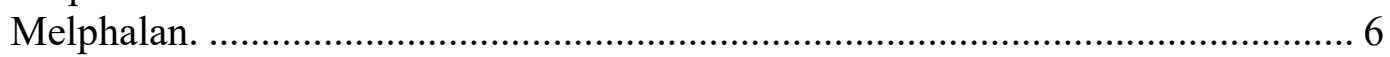

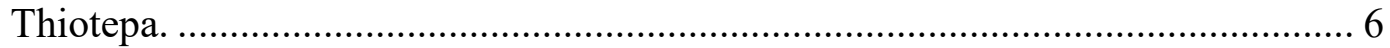

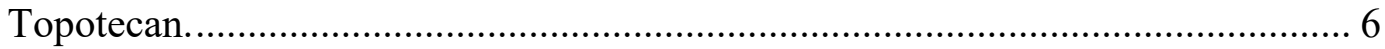

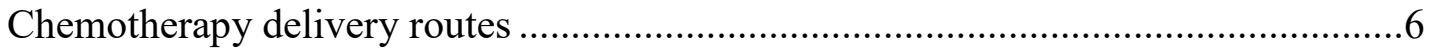

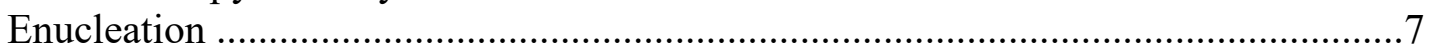

Immune ablation and autologous stem cell therapy (ASCT) ...................................

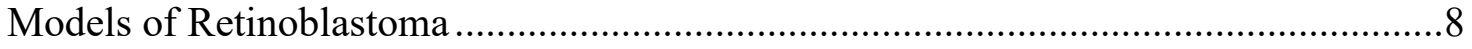

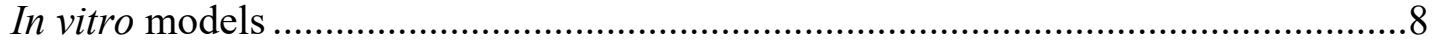

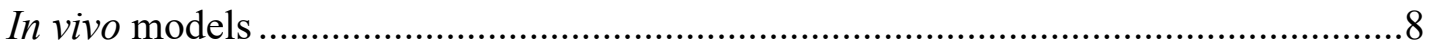

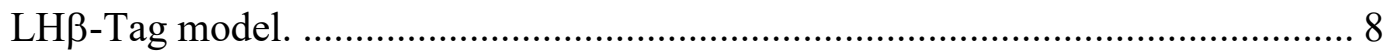

Genetic knock-out and knock-in models. ………................................................ 8

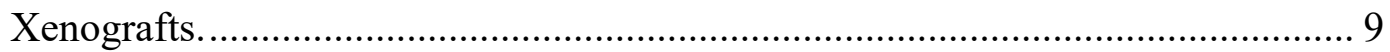

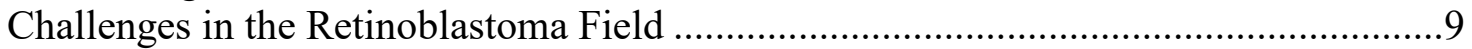

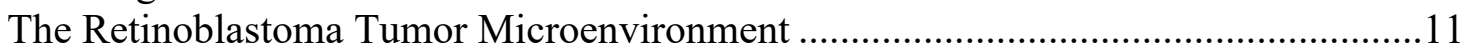

The tumor microenvironment of endophytic and exophoytic $\mathrm{Rb}$.............................11

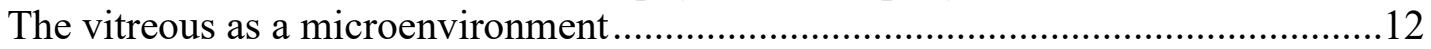

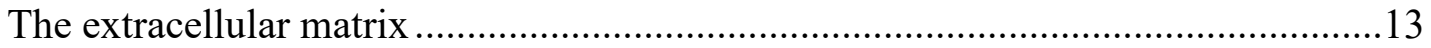

Matrix metalloproteinases in the healthy and diseased eye................................ 13

Matrix metalloproteinases in cancer............................................................. 13

The Platelet-Derived Growth Factor Family ............................................................

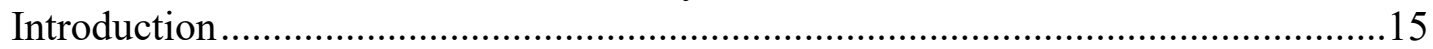

Platelet-derived growth factors in ocular health and disease.....................................16 
Platelet-derived growth factors in cancer.

Vascular endothelial growth factor, a sub-family of platelet-derived growth factor family

Rationale and Specific Aims.

\section{CHAPTER 2. RESULTS I: INHIBITION OF MMP-2 AND MMP-9 DECREASE CELLULAR MIGRATION, AND ANGIOGENESIS IN IN VITRO

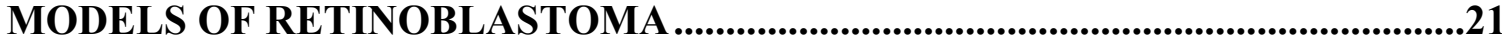

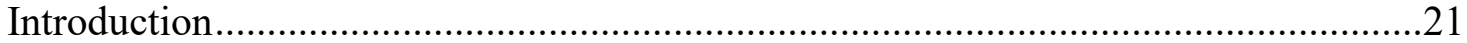

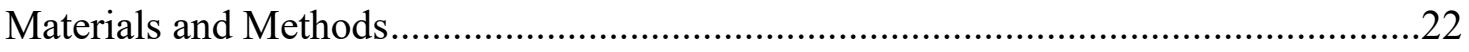

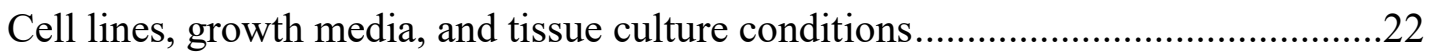

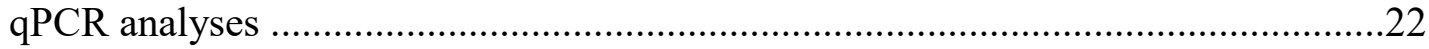

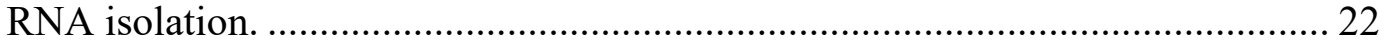

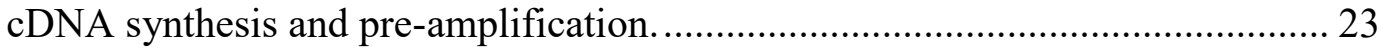

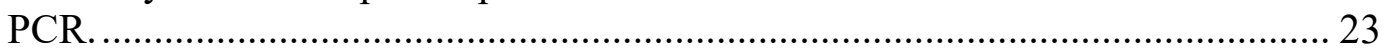

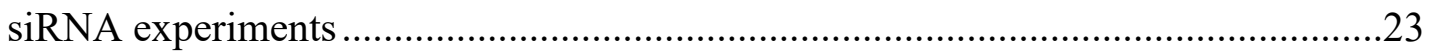

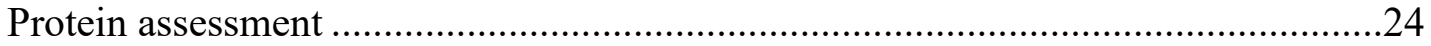

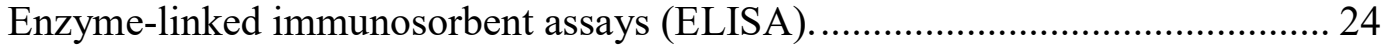

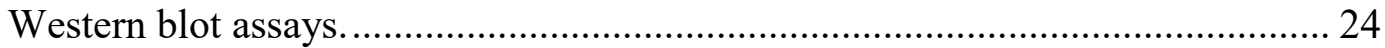

Bicinchoninic acid (BCA) protein concentration assay .................................. 24

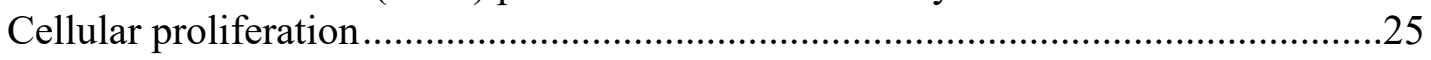

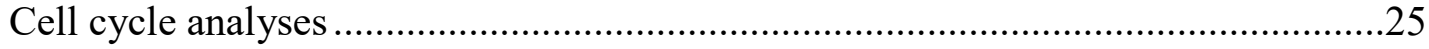

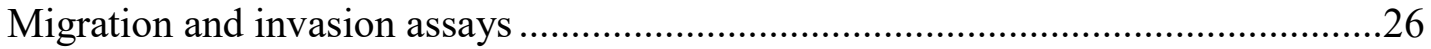

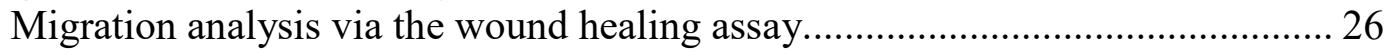

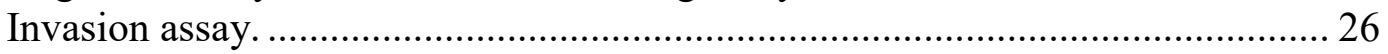

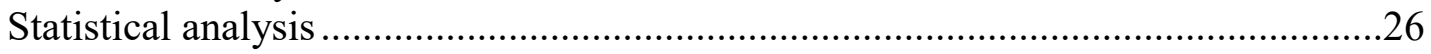

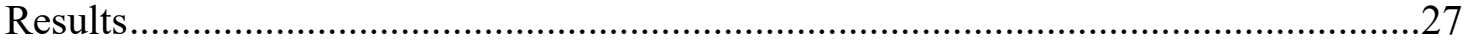

Inhibition of MMP-2 and MMP-9 decreases migration in the metastatic $\mathrm{Y} 79 \mathrm{Rb}$ cell line, and viability in the non-metastatic Weri-1 model.................................27

Downregulation of MMP-2 and MMP-9 by pharmacological inhibitors in Y79

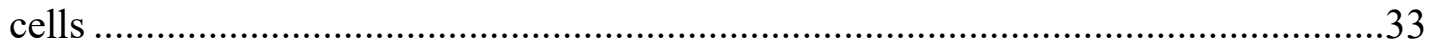

Pharmacological inhibition of MMPs reduces secretion of angiopoietin-2, but

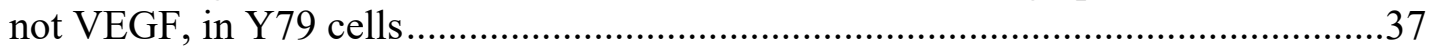

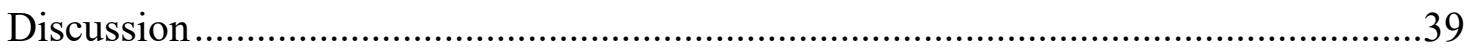

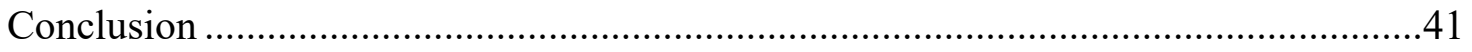

\section{CHAPTER 3. RESULTS II: TARGETING THE PLATELET-DERIVED GROWTH FACTOR-BETA STIMULATORY CIRCUITRY TO CONTROL

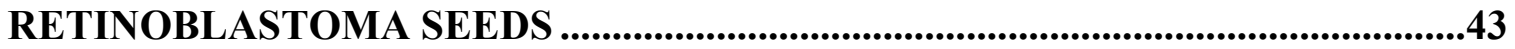

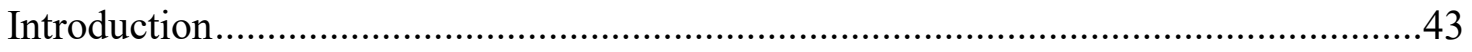

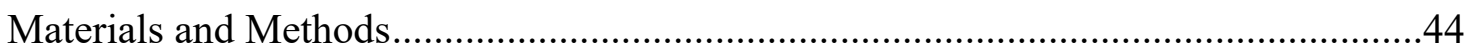

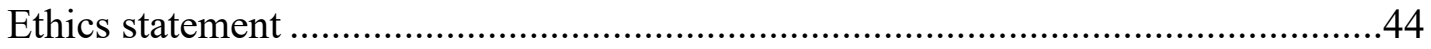

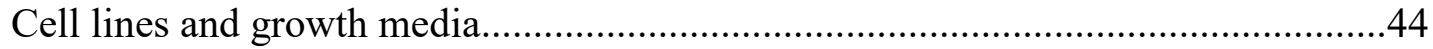

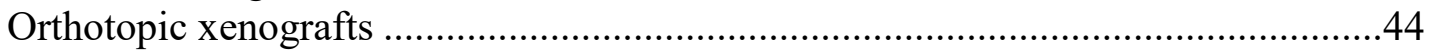

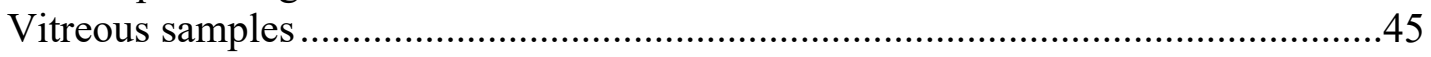

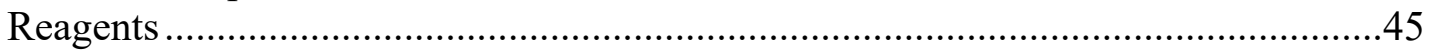


Immunohistochemistry (IHC) .........................................................................4

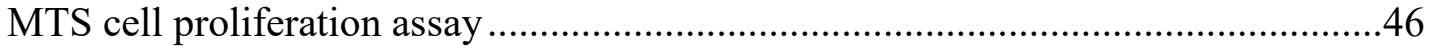

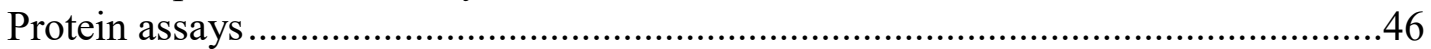

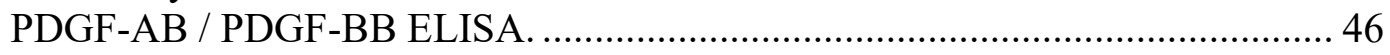

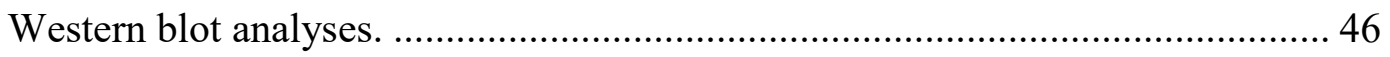

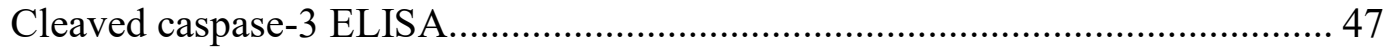

Bicinchoninic acid (BCA) protein concentration assay ........................................ 47

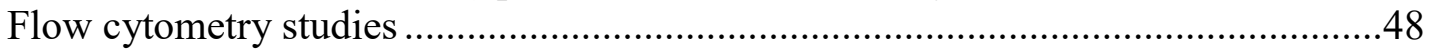

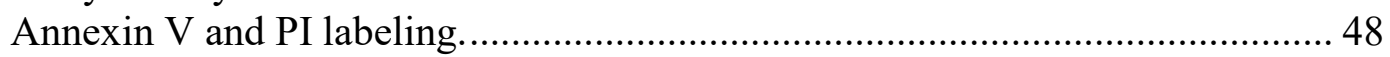

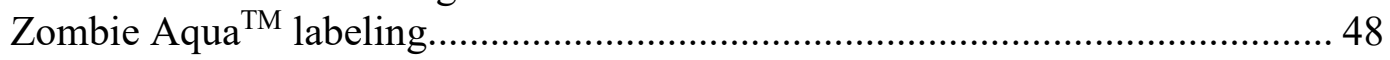

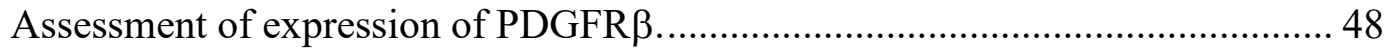

p65 NFkB Nuclear Translocation by imaging flow cytometry. ............................ 48

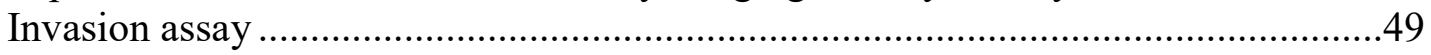

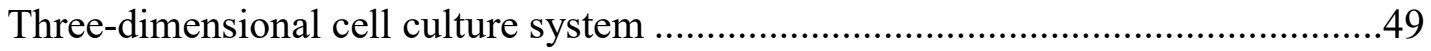

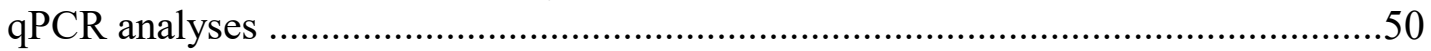

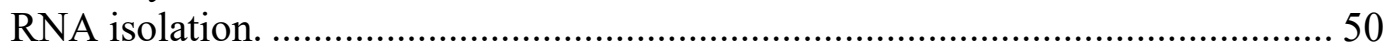

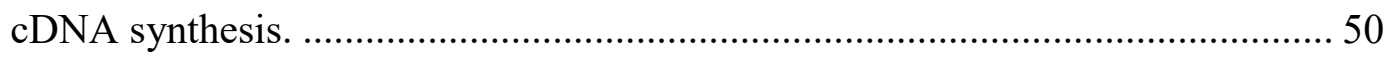

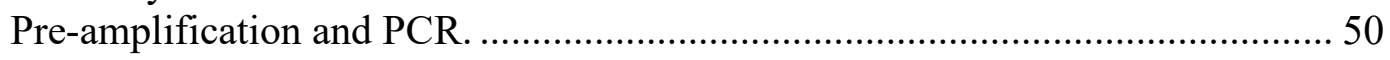

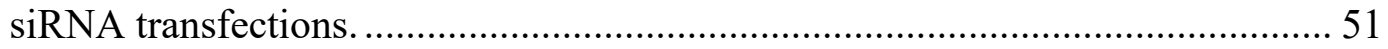

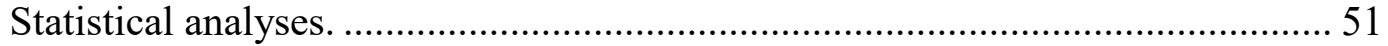

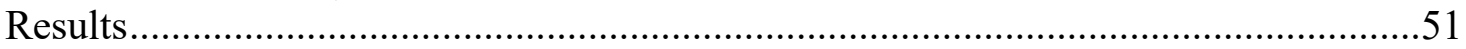

Expression of the Platelet-Derived Growth Factor Receptor signaling network in retinoblastoma tumor and tumor-derived cell lines ...................................................51

Pharmacological disruption of PDGFR $\beta$ signaling reduced Rb proliferation,

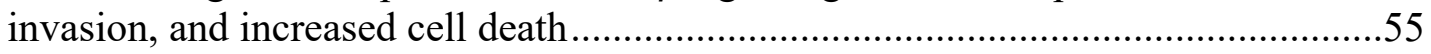

Disruption of PDGFR $\beta$ targets the MDM2 and NFkB signaling cascades ...............57

Reduction of Rb spheroids by IM in a 3D cell culture model of Retinoblastoma vitreous seeds .................................................................................................64

Reduction in cell viability, MDM2 signaling and p65 nuclear translocation are

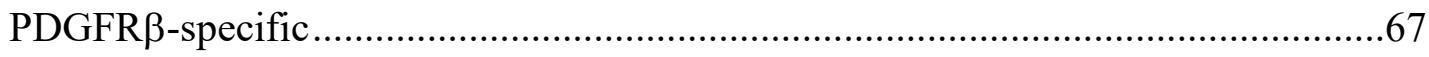

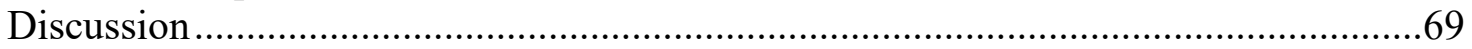

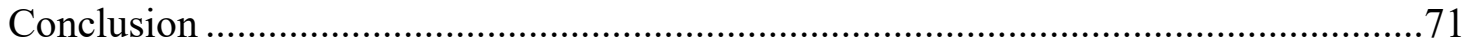

\section{CHAPTER 4. RESULTS III: ANTI-PLATELET-DERIVED GROWTH} THERAPY AS A POTENTIAL TARGET TO DECREASE RESISTANCE ............72

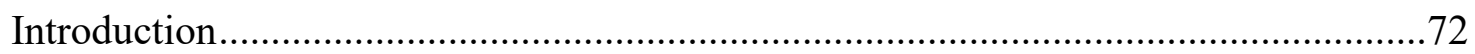

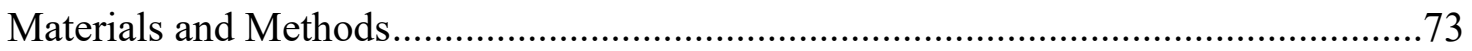

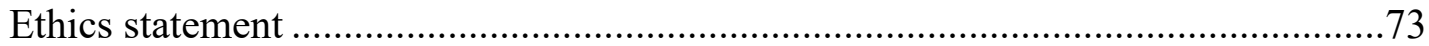

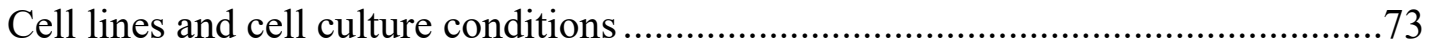

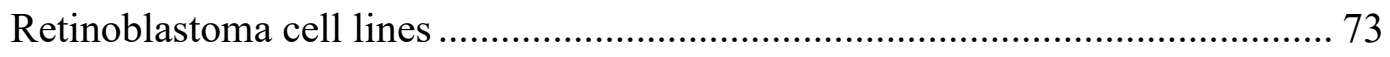

Breast cancer cell line. .................................................................................. 73

Primary human retinal microvascular endothelial cells (hRECs, ACBRI 181).... 73

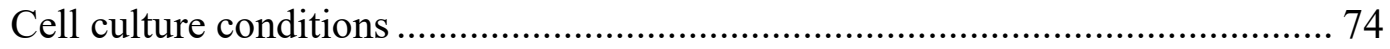

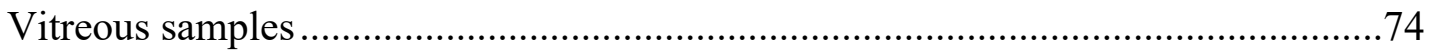

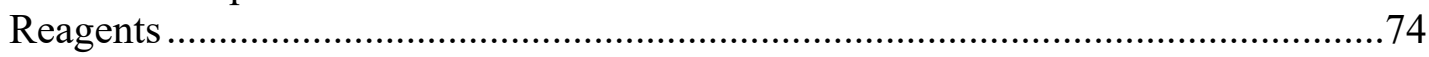

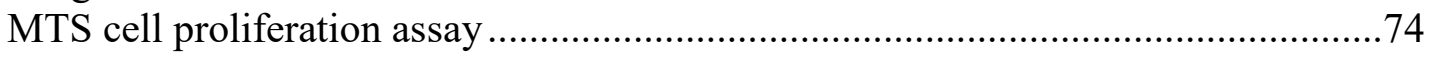




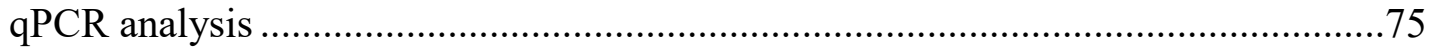

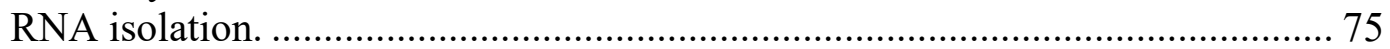

cDNA synthesis and pre-amplification......................................................... 75

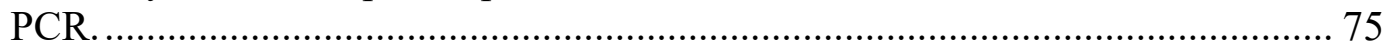

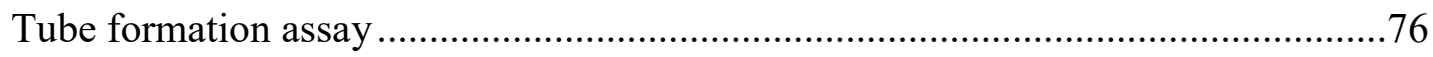

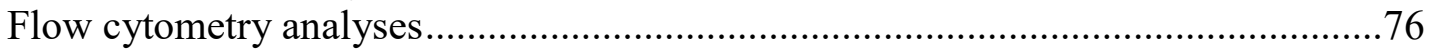

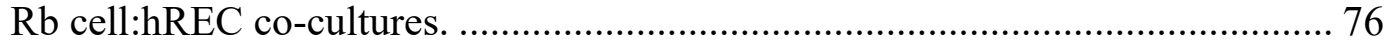

Assessment of efflux activity......................................................................... 76

Assessment of co-expression of the pPDGFR $\beta$ and CD44 proteins...................... 76

Results...............................................................................................................

Proliferation of primary human retinal endothelial cells is independent of PDGF-

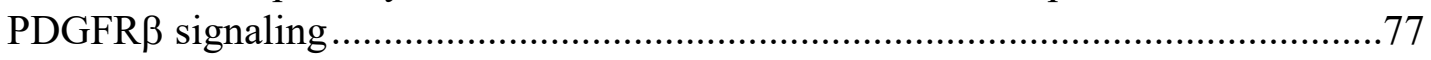

Disruption of the PDGF-PDGFR signaling pathway does not affect angiogenic

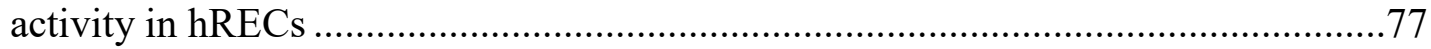

Reduction in hREC VEGF production is PDGFR $\beta$-independent ..............................79 Expression of ATP-binding cassette (ABC) transporter family in the Rb vitreous

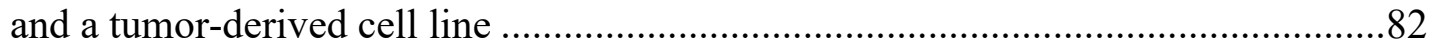

Disruption of PDGF-PDGFR $\beta$ signaling reduces ABC transporter efflux ................82 $\mathrm{CD} 44$ is overexpressed in the naïve $\mathrm{Rb}$ vitreous and is by PDGF-PDGFR $\beta$

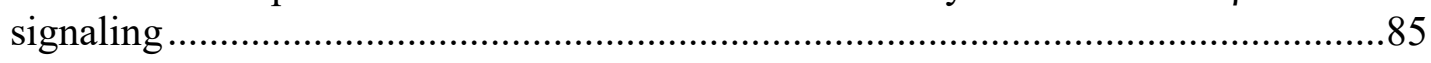

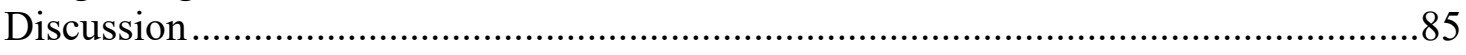

CHAPTER 5. DISCUSSION ..............................................................................................91

LIST OF REFERENCES .................................................................................................97

APPENDIX. TABLES OF PRIMERS AND ANTIBODIES USED............................116

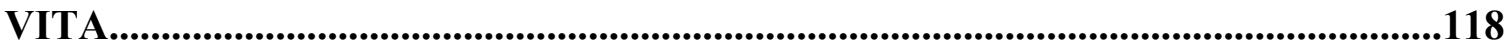




\section{LIST OF FIGURES}

Figure 1-1. Genetic knockout models of retinoblastoma..................................................10

Figure 1-2. Members of the Matrix Metalloproteinase (MMP) Family ...........................14

Figure 1-3. Members of the PDGF family and their receptors........................................17

Figure 2-1. Inhibition of MMP-2 and MMP-9 reduced Rb invasion.............................28

Figure 2-2. Inhibition of MMP-2 and MMP-9 reduced Rb cell migration........................30

Figure 2-3. Inhibition of MMP-2 or MMP-9 reduced Rb cell viability and cell cycle

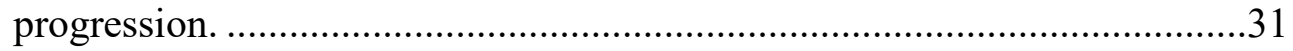

Figure 2-4. Weri-1 Rb cells are sensitive to MMPIs.......................................................32

Figure 2-5. Pharmacological inhibitors of MMP-2 and MMP-9 downregulate MMP2

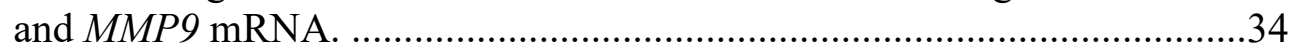

Figure 2-6. MMP inhibition reduces angiogenic protein levels. ......................................38

Figure 2-7. Working model of the roles of MMP-2 and MMP-9 in retinoblastoma cells.

Figure 3-1. Immunohistochemistry of the active and non-active forms of PDGFR $\beta$ in $\mathrm{Rb}$.

Figure 3-2. Expression of the key PDGF family members and the PDGFR $\beta$ in Rb.......54

Figure 3-3. Abundance of PDGF ligands in the vitreous of Rb patients..........................56

Figure 3-4. Imatinib mesylate disrupts PDGF-PDGFR $\beta$ signaling..................................56

Figure 3-5. PDGF-PDGFR $\beta$ signaling induces $\mathrm{Rb}$ cell proliferation and regulates survival.

Figure 3-6. Reduction in invasive capacity of Y79 cells after IM treatment on basement membrane-coated inserts.

Figure 3-7. PDGF-PDGFR $\beta$ signaling regulates cell survival signaling. 60

Figure 3-8. Lack of VEGF-VEGFR2 compensatory mechanism when PDGFPDGFR $\beta$ signaling is disrupted.

Figure 3-9. Disrupting PDGF-PDGFR $\beta$ signaling decreases NFkB nuclear .63 
Figure 3-10. Three-dimensional magnetic tumor spheroids as an in vitro model for vitreous seeds.

Figure 3-11. Quantitative and qualitative assessment of first in vitro model of vitreous seeds.

Figure 3-12. Confirmation of PDGFR $\beta$-specific effects in Rb cells by PDGFRB siRNA. .68

Figure 4-1. hREC proliferation occurs independent of PDGF-PDGFR $\beta$ signaling. .......78

Figure 4-2. PDGF-PDGFR $\beta$ signaling is unable to initiate tube formation. ...................80

Figure 4-3. PDGF-PDGFR $\beta$ signaling is not required for hREC tube formation...........80

Figure 4-4. Treatment with IM reduces hREC VEGF production. ..............................81

Figure 4-5. Expression of the $\mathrm{ABC}$ transporters in naïve human $\mathrm{Rb}$ vitreous. ..............83

Figure 4-6. Expression of the ABC transporters in Y79 cell lines is not altered by PDGF-PDGFR $\beta$ signaling.......................................................... 83

Figure 4-7, ABC transporter efflux activity is low in Y79 Rb cells............................84

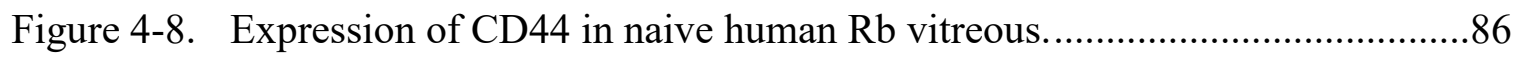

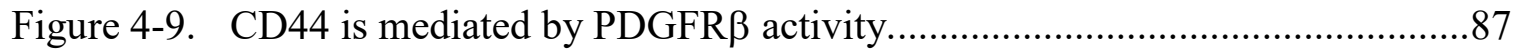

Figure 5-1. Working model of vitreous seed microenvironment. ...............................96 


\section{LIST OF ABBREVIATIONS}

\begin{tabular}{|c|c|}
\hline $\mathrm{ABCB} 1$ & ATP Binding Cassette Subfamily B Member 1 gene \\
\hline $\mathrm{ABCC} 1$ & ATP Binding Cassette Subfamily C Member 1 gene \\
\hline $\mathrm{ABCC} 2$ & ATP Binding Cassette Subfamily C Member 2 gene \\
\hline ABCG2 & ATP Binding Cassette Subfamily G Member 2 gene \\
\hline$a k a$ & also known as \\
\hline $\mathrm{APC}$ & Allophycocyanin \\
\hline $\mathrm{ASCT}$ & Autologous stem cell therapy \\
\hline BCRP & Breast cancer resistance protein \\
\hline $\mathrm{BRB}$ & Blood-Retinal barrier \\
\hline CD44 & Cluster of differentiation $44, a k a$ hyaluronan receptor \\
\hline CD133 & Cluster of differentiation 133, aka Prominin 1 \\
\hline cDNA & Complementary deoxyribonucleic acid \\
\hline CFSE & Carboxyfluorescein succinimidyl ester \\
\hline CNS & Central nervous system \\
\hline EBRT & External beam radiotherapy \\
\hline EMT & Epithelial-to-mesenchymal transition \\
\hline GIST & Gastrointestinal stromal tumors \\
\hline hREC & primary human retinal microvascular endothelial cells \\
\hline hUVEC & primary human umbilical vein endothelial cells \\
\hline IAC & Intraarterial chemotherapy \\
\hline $\mathrm{IHC}$ & Immumohistochemistry \\
\hline IM & Imatinib mesylate (marketed as Gleevec) \\
\hline
\end{tabular}


IVC

IViC

MDM2

MDM4

MDMX

MDR1

MRP1

MRP2

MMP

$M M P 2$

MMP-2

MMP9

MMP-9

MMPI

$\mathrm{NF \kappa B}$

PDGF

PDGFA

PDGFR $\alpha$

PDGFRA

PDGFB

PDGFR $\beta$

PDGFRB

PDGFR $\alpha \beta$
Intravenous chemotherapy

Intravitreal chemotherapy

Mouse double minute homolog 2

Mouse double minute homolog 4; also referenced as MDMX

Interchangeable with MDM4

Multi-drug resistant protein 1, P-glycoprotein (P-gp)

Multi-drug resistance-associated protein 1

Multi-drug resistance-associated protein 2

Matrix metalloproteinase

Matrix metalloproteinase 2 gene

Matrix metalloproteinase 2 protein

Matrix metalloproteinase 9 gene

Matrix metalloproteinase 9 protein

Matrix metalloproteinase inhibitor

Nuclear factor kappa-light-chain-enhancer of activated B cells

Platelet-derived growth factor

Platelet-derived growth factor A (homodimer) protein

Platelet-derived growth factor receptor alpha (homodimer)

Platelet-derived growth factor receptor alpha gene

Platelet-derived growth factor B (homodimer) protein

Platelet-derived growth factor receptor beta (homodimer)

Platelet-derived growth factor receptor beta gene

Platelet-derived growth factor receptor alpha (heterodimer) 


\begin{tabular}{|c|c|}
\hline PDGFAB & Platelet-derived growth factor $\mathrm{AB}$ (heterodimer) protein \\
\hline PDX & Patient-derived xenograft \\
\hline $\mathrm{pRb}$ & Retinoblastoma protein \\
\hline $\mathrm{Rb}$ & Retinoblastoma disease \\
\hline $\mathrm{Rb}: \mathrm{hREC}$ & Ratio of $\mathrm{Rb}$ cells to hRECs \\
\hline$R B 1$ & Retinoblastoma gene \\
\hline rhPDGF & Recombinant human PDGF-BB \\
\hline RNA & Ribonucleic acid \\
\hline RTK & Receptor tyrosine kinase \\
\hline siRNA & Small interfering RNA \\
\hline SSIOAC & Super-selective intra-ophthalmic artery chemotherapy \\
\hline TKI & Tyrosine kinase inhibitor \\
\hline TME & Tumor microenvironment \\
\hline VEGF & Vascular endothelial growth factor \\
\hline VEGFR & Vascular endothelial growth factor receptor \\
\hline
\end{tabular}




\section{CHAPTER 1. INTRODUCTION AND BACKGROUND}

Retinoblastoma $(\mathrm{Rb})$ is the most common intraocular malignancy in children ${ }^{1}$. To date, few studies have attempted to examine how the tumor interacts with its surroundings, called the tumor microenvironment (TME). This dissertation seeks to reveal how these crucial interactions promote tumor survival and, thereby, allow introduction of targeted therapies for clinical use.

\section{History of Retinoblastoma Disease}

Retinoblastoma was first described in a case report by anatomist Pieter Pauw (also referred to as Petrus Pawius) in 1597 as a "an enormous tumor out of the left eye, until the untouched eyeball with all muscles protruded out, and had grown into such a mass that the protuberance had acquired the size of two fists"2. Two centuries later, in 1809, James Waldrop characterized these intraocular masses (known today as retinoblastoma) as fungus haematodes and recommended removal of the afflicted eye ${ }^{3}$. The tumor was first characterized as a glioma of the retina in 1864 by the German physician-scientist, Rudolf Virchow ${ }^{4}$. However, pathologist Simon Flexner and the Austrian ophthalmologist Hugo Wintersteiner noticed the formation of rosettes and described the tumor as neuroepithelioma ${ }^{4}$. Finally, in the 1920 s, ophthalmic pathologist Frederick Herman Verhoeff noted the primitiveness of the tumor and the similarity to that of undifferentiated retinae, thus coining the term 'retinoblastoma' ${ }^{5}$. In the 1960 s, as treatment options began to advance, a classification system was needed to better understand outcomes and therapeutic effectiveness. Drs. Algernon Reese and Robert Ellsworth classified tumors based on rates of both ocular and vision salvage to radiation called the Reese-Ellsworth classification ${ }^{6,7}$. With chemotherapy slowly replacing radiation, a new classification system was developed in 2003 based on responses to chemotherapy, named the International Classification of Retinoblastoma ${ }^{8,9}$. Today, more is known about genetics of retinoblastoma and, by further understanding of this small subset of tumors, great leaps have been made in the fields of both genetics and oncology.

\section{Genetics of Retinoblastoma Disease}

\section{Primary genetic alterations}

In 1971, physician-scientist Alfred Knudson postulated that multiple mutations were necessary for tumorigenesis and the hereditary basis for which cancer could arise ${ }^{10}$. This hypothesis is known as the "two hit hypothesis". Using Rb as a model for his hypothesis, Knudson compared incidences, ages at time of diagnosis, number of eyes afflicted, and familial history of the disease. The results demonstrated first, patients with both eyes affected (bilateral disease) were younger and had a genetic predisposition to $\mathrm{Rb}$ while those patients with one affected (unilateral disease) were older and did not have a 
family history of $\mathrm{Rb}^{10}$. Knudson concluded when comparing the two outcomes, that $\mathrm{Rb}$ required two mutations for tumorigenesis. The bilateral (or hereditary) form of the disease was caused by one germline mutation and a second, later mutation that occurred in somatic cells; meanwhile, patients with unilateral (non-hereditary) form of Rb required two mutations in somatic cells, thus the discrepancy in ages of diagnosis. Ultimately, this work proved crucial in the understanding of tumorigenesis and the origins of cancer.

Within the next decade, researchers had begun attempting to identify and locate the gene involved in $\mathrm{Rb}$ development. In 1984, the $R B 1$ gene was first identified on chromosome 13q14.2 and was then subsequently cloned - the first time a tumor suppressor had been cloned ${ }^{11,12}$. The $R B 1$ gene encodes the sequence for the retinoblastoma protein $(\mathrm{pRb})$, a member of the pocket protein family found to be involved in a number of cellular events most notably in cell cycle progression ${ }^{13} \cdot \mathrm{pRb}$ was initially thought to exist in two forms: (1) hypophosphorylated and (2) hyperphosphorylated, Mechanistically, depending on the level of phosphorylation $\mathrm{pRb}$ could bind the cell cycle and DNA synthesis E2F transcription factors (hypophosphorylated) or it could not (hyperphosphorylated) ${ }^{14,15}$. Yet, subsequent work has invalidated this hypothesis as pRb can exist in (at least) three states: (1) non-phosphorylated, (2) monophosphorylated, and (3) hyperphosphorylated, each with different respective function $\left.(s)^{16}: 1\right)$ non-phosphorylated $\mathrm{pRb}$ has been associated with cellular arrest, or senescence, and exit of the cell cycle ${ }^{17} ; 2$ ) monophosphorylated $\mathrm{pRb}$ has been readily detected in early G1 phase of cell cycle and prevents withdrawal from the cell cycle ${ }^{16}$; finally, 3) hyperphosphorylated $\mathrm{pRb}$ still lacks the ability to bind to E2F and, therefore, promotes cell cycle progression. Then, in the absence of $\mathrm{pRb}$ (such as mutations within the $R B 1$ gene), E2F transcription factors are unbound and free to induce DNA synthesis and cell division. This cell division may occur at a rapid rate, resulting in tumor formation, as tumors are the result of uncontrolled proliferation. $R B 1$ and the protein which it encodes, have become increasingly detected in a number of malignancies, including lung, breast, and brain cancers ${ }^{18-20}$. More than $95 \%$ of $\mathrm{Rb}$ cases harbor mutations or promote methylation of $R B 1$. However, $1-2 \%$ of $\mathrm{Rb}$ cases present with wild type $R B 1\left(R B 1^{+/+}\right)^{21}$ and these tumors possess amplification of the proto-oncogene $M Y C N^{22}$.

\section{Secondary genetic alterations}

While alterations in $R B 1$ are necessary for $\mathrm{Rb}$ formation, work has shown that $R B 1$ loss is insufficient to drive $\mathrm{Rb}$ tumorigenesis. When $R B 1$ is lost $\left(R B 1^{+/-}\right.$or $\left.R B 1^{-/-}\right)$, a benign tumor, called 'retinoma', develops ${ }^{23}$. One prevailing reason for this benign tumor formation is that unlike many other malignancies, $\mathrm{Rb}$ tumors harbor wild type $\mathrm{p} 53$. If $R B 1$ is inactivated, tumor cells are able to withdraw from the cell cycle and undergo programmed cell death, or apoptosis ${ }^{24}$. Previous work by Dyer and colleagues, identified amplification of two inactivators of $\mathrm{p} 53$, mouse double minute homolog 2 (MDM2) and murine double minute $\mathrm{X}$ ( $M D M X$, sometimes referenced as $M D M 4)$ in $\mathrm{Rb}$ and found upon amplification of either gene, p53-mediated cell death is inhibited and $\mathrm{Rb}$ tumors are formed $^{24}$. Genetic analyses of fetal retinae, retinomas, and retinoblastomas have 
illustrated other genes in which secondary mutations synergize with $R B 1$ mutations to cause $\mathrm{Rb}$ tumor development including: $K I F 14, D E K, E 2 F 3$, and $C D H 11$, as well as mutations causing loss of expression of key tumor suppressor proteins $\mathrm{p} 16^{\mathrm{INK} 4 \mathrm{a}}$ and $\mathrm{p} 130$ that result in $\mathrm{Rb}$ when combined with an $R B 1$ mutation $^{23}$. Conflicting studies exist about the direct roles these genes may play in $\mathrm{Rb}$ tumorigenesis ${ }^{25}$. A number of studies have illustrated when comparing $\mathrm{Rb}$ to other malignancies, the genome of $\mathrm{Rb}$ is quite stable $^{26,27}$. For instance, when conducting genetic analyses in a large cohort of samples, 199 genes were identified that were unique to single tumors, demonstrating the vast heterogeneity of $\mathrm{Rb}$ tumors ${ }^{26}$. Further analyses have begun to investigate the epigenome of $\mathrm{Rb}$ and discovered while the $\mathrm{Rb}$ genome may be quite stable, the epigenome is anything but, finding a number of genes deregulated epigenetically ${ }^{27}$.

\section{Pathophysiology of Retinoblastoma}

\section{Incidence}

$\mathrm{Rb}$ is the most common intraocular cancer in children, but still is considered a rare pediatric malignancy with approximately 8,000 new cases presented every year worldwide $^{28}$. The annual tumor incidence is approximately 1 in 16,000-18,000 live births and shows higher incidence in less industrialized nations ${ }^{28}$. Annually, there are between $200-300$ new cases of Rb diagnosed in the United States ${ }^{5}$. China and India, the most populous countries in the world, see approximately 1,000 new cases every year ${ }^{29}$. Clinically, $\mathrm{Rb}$ is seen almost exclusively in children $<5$ years old ${ }^{30}$. When examining incidence of unilateral (one eye) vs. bilateral (both eyes), approximately $60-70 \%$ of $\mathrm{Rb}$ patients will present with unilateral disease while $30-40 \%$ of patients will present with bilateral disease ${ }^{30,31}$. Normally, the two main symptoms of $\mathrm{Rb}$ are leukocoria, a reflection of light by a white mass, and crossing of the eyes, or strabismus ${ }^{28}$. The average age at time of diagnosis are 24 months for patients with unilateral disease and 13 months for those with bilateral disease ${ }^{30}$. Patient statistics have not illustrated predisposition in $\mathrm{Rb}$ development among any specific race or gender ${ }^{4}$.

Socioeconomic factors play an important role in $\mathrm{Rb}$ diagnosis, care, and, ultimately, survival. When comparing worldwide incidence of Rb, the United States, Europe, and Australia harbor relatively low percentages of those afflicted with $\mathrm{Rb}(\sim$ $11 \%$ of cases), most patients that are affected with the disease are in middle-income countries $(\sim 69 \%)$ or low-income countries $(\sim 20 \%)^{28}$. The two main factors associated with survival of $\mathrm{Rb}$ are presence of physicians and human development index, a metric that considers multiple factors including education, life expectancy, and wealth ${ }^{32}$. Patients in countries with less-developed, poorer infrastructure typically have longer lag times that delay onset of diagnosis and provide a worse prognosis for survival ${ }^{33}$. Unfortunately, metastasis and survivability align with income disparities across the globe. Rates of metastasis and survivability in low-income countries are $32 \%$ and $40 \%$, respectively. Meanwhile, middle-income countries experienced rates of approximately $10 \%$ and $78 \%{ }^{32}$. In developed countries, such as the United States, Rb is the most 
survivable pediatric malignancy ${ }^{3}$. Given these discrepancies, there has been a large outreach to support global health initiatives in treating retinoblastoma.

\section{Primary tumor subtypes}

Currently, there are five main growth patterns identified within Rb: endophytic, exophytic, mixed (both endophytic and exophytic), necrotic, and diffuse infiltrating.

Endophytic. The endophytic growth pattern of $\mathrm{Rb}$ is one whereby the tumor begins growing into the vitreous cavity. These tumors are often vascularized but small pockets of tumor cells, called vitreous seeds, that break off from the primary tumor inhabit the vitreous cavity ${ }^{5}$.

Exophytic. Exophytic Rb tumors typically grow away from the vitreous cavity and into the retina. These tumors can invade the choroid, the vasculature of the eye and normally present as retinal detachments. Similar to vitreous seeds, small pockets of free floating tumors can survive in the subretinal space (subretinal seeds) ${ }^{34}$.

Mixed. Tumors with growth patterns termed mixed are, as the name implies, a mixture of both endophytic and exophytic growth patterns.

Necrotic. Necrotic Rb growth pattern consists of high levels of necrotic tissue ( $>$ $90 \%$ necrosis). This necrosis is not limited to tumor cells and may include nearby surrounding tissue (i.e. retina) ${ }^{35}$.

Diffuse infiltrating. The rarest of the $\mathrm{Rb}$ growth patterns (comprising just $2 \%$ of classified tumors), diffuse infiltrating tumors invade both the retina and vitreous cavity without any large, primary mass. These tumors exhibit unique growth patterns and can form small pockets of tumors in the anterior chamber in a rare occurrence called anterior chamber seeding ${ }^{36}$.

\section{Metastatic retinoblastoma}

Without medical attention and treatment, $\mathrm{Rb}$ will invade surrounding tissue and can metastasize via a number of different anatomical pathways. The most prevalent route of metastasis is through the optic nerve fiber bundles into orbital portion of the optic nerve and then into the brain or into circulation of subarachnoid fluid that results in metastatic sites into distant parts of the brain, spinal cord and opposite optic nerve ${ }^{37}$. Secondly, Rb may spread through the choroid in a process called haematogenous dissemination. Haematogenous dissemination of $\mathrm{Rb}$ leads to invasion of the orbital bones, lungs, brain, and surrounding bone as well as access to lymphatics ${ }^{37}$. Similarly, Rb tumors can disseminate through lymphatics into regional lymph nodes ${ }^{37-39}$. As is the case with all cancers, metastasis carries a poorer prognosis for survival ${ }^{40}$. 


\section{Treatment of Retinoblastoma}

Proper management and treatment of $\mathrm{Rb}$ is an ongoing topic. Options for treatment are often dependent on tumor size, location, and patient outcome. The goals when treating $\mathrm{Rb}$ are as follows: 1) save the child's life, 2) salvage the globe, and 3) preserve vision. The first goal of saving lives is well met in developed countries (less so in developing countries). However, both globe salvage and vision preservation are still clinically challenging. The following section will seek to discuss the current methods of management and treatment options in $\mathrm{Rb}$.

\section{Cryotherapy and thermotherapy}

Cryotherapy and thermotherapy have both been used within the clinic and have shown some success. Cryotherapy works by freezing $\left(-80^{\circ} \mathrm{C}\right)$ tumor tissue, leading to ice crystal formation within the cell membrane, and cell lysis. However, cryotherapy is most commonly applied to small, anterior tumors in three, rapid freeze-thaw cycles ${ }^{41}$.

While cryotherapy involves freezing of the tumor, thermotherapy is the opposite in that the tumor is heated. Commonly, thermotherapy utilizes either an argon laser $(532 \mathrm{~nm})$ or diode transpupillary / transscleral laser $(810 \mathrm{~nm})$ with each laser possessing different utility. The argon laser is utilized to target and heat the tumor-supporting blood supply to temperatures above $60^{\circ} \mathrm{C}$ for photocoagulation ${ }^{42}$. The diode laser is used to heat tissue at temperatures between $45^{\circ} \mathrm{C}$ and $60^{\circ} \mathrm{C}$ (below photocoagulation temperatures) and is capable of inducing tumor clearance ${ }^{43,44}$. Both cryotherapy and thermotherapy can be utilized for smaller tumors with minimal complications.

\section{Radiation}

One of the most successful forms of treatment for $\mathrm{Rb}$ is the use of radiation to target the tumor. The advent of radiotherapy in $\mathrm{Rb}$ came at a time when removal of the affected eye (or eyes) was seen as the only therapeutic option ${ }^{45}$. Nearly 50 years ago, external beam radiotherapy (EBRT) became the standard treatment for $\mathrm{Rb}$ and patients with advanced intraocular disease had higher rates of ocular salvage instead of undergoing enucleation ${ }^{46}$. Yet, there has been a dramatic decrease in the use of EBRT in $\mathrm{Rb}$ as it exposes the pediatric population to significantly higher risk of secondary malignancies throughout their lives as has been extensively documented over the past 40 years ${ }^{47-50}$. Despite this knowledge, EBRT remains a therapeutic option for patients with bilateral, advanced intraocular disease in the hopes of sparing at least one eye.

The introduction of newer treatment modalities have allowed for safer, targeted radiotherapies including that of proton beam therapy that shows strong tumor control without the complications associated with EBRT ${ }^{51}$. Another therapy, called episcleral plaque brachytherapy (or simply brachytherapy) involves the placement of radioactive 
plaques on to the sclera ${ }^{52}$. These plaques are typically loaded with radioactive Iodine-125 $\left({ }^{125} \mathrm{I}\right)$ sewn to the sclera directly over the tumor, minimizing damage to the surrounding tissue. Other isotopes may be used, such as Ruthenium-106 $\left({ }^{106} \mathrm{R}\right)$.

\section{Chemotherapy}

The current standard of care in managing and treating $\mathrm{Rb}$ is the use of chemotherapy. Often, patients presenting with bilateral $\mathrm{Rb}$ are given a chemotherapeutic regimen to help cytoreduce (or chemoreduce) tumor burden within the eye. Despite this, a single chemotherapeutic agent cannot be used to salvage all eyes ${ }^{53}$. Combination chemotherapy increases the chance to eliminate tumor cells undergoing different stages of cell cycle due to their differences in mechanisms of action. Rb patients will often be treated with a combination of chemotherapies, most notably the combination of carboplatin, vincristine, and etoposide (see below). Other chemotherapeutic agents, such as cyclophosphamide (induces DNA crosslinking) and doxorubicin (similar mechanism as etoposide), may also be used ${ }^{54,55}$. The three most commonly used agents all possess different mechanisms of action:

Carboplatin. Carboplatin is a platinum-based alkylating agent that, upon uptake, acts on nucleophilic molecules and induces damage in DNA that ultimately result in apoptosis $^{56}$.

Vincristine. In a similar manner, vincristine works by binding tubulin, a key molecule involved in microtubule polymerization in cell cycle ${ }^{57}$.

Etoposide. Etoposide is an effective chemotherapy aimed at inhibiting topoisomerase II, which plays a role in DNA ligation. By addition of etoposide, DNA ligation does not occur and there is an increase in DNA strand breaks that leads to cell death $^{58}$.

Melphalan. Melphalan is a nitrogen mustard alkylating agent, similar to carboplatin with DNA damage being an end result of melphalan exposure ${ }^{59}$.

Thiotepa. Thiotepa, another alkylating agent, works similarly to both carboplatin and melphalan and induces DNA damage.

Topotecan. Topotecan is a topoisomerase I inhibitor that induces large numbers of both single strand and double strand breaks in DNA that lead to apoptosis.

\section{Chemotherapy delivery routes}

There are multiple modes of delivery for chemotherapy. Method(s) of administration are decided based on individual case presentation of unilateral or bilateral, tumor size, location(s) of tumor, patient age, family history, and potential outcomes. The 
most common method of chemotherapy delivery is intravenously (IVC). Normally, IVC administration of carboplatin, vincristine, and etoposide are used as a first-line therapy for treatment of $\mathrm{Rb}$ and are capable of salvaging eyes afflicted with advanced intraocular disease $^{60}$. IVC chemotherapy has proven quite safe for the pediatric population and places patients at a much lower risk for development of secondary malignancies when compared to $\mathrm{EBRT}^{61}$. However, many chemotherapeutics have reduced penetration through the blood-retinal barrier (BRB) and may not reach cytotoxic levels ${ }^{55}$. Therefore, newer, more focal modes of delivery have been investigated.

One of these newer techniques is intra-arterial chemotherapy (IAC). In this method of delivery, chemotherapy is administered through the ophthalmic artery to achieve higher, more tumoricidal concentrations. Most commonly, melphalan is administered through IAC. Reports have claimed IAC is vastly superior to IVC with reduced toxicities and increased globe salvage ${ }^{62}$. Yet, reports have arisen regarding toxicities dampening the enthusiasm for IAC ${ }^{63-65}$.

As both IVC and IAC could potentially be impeded by the BRB focus has shifted toward local delivery to the eye to increase cytotoxic concentrations in the vitreous to treat seeding. Local delivery could be to the anterior part of the eye, being subconjunctival (or subtenon) and intravitreal (IViC) the most common. Subconjunctival administration is done into the subconjunctival space whereby high levels of chemotherapy readily penetrate into the affected area(s ${ }^{66}$. Ongoing clinical trials are studying the effectiveness of subconjunctival carboplatin combined with systemic topotecan $^{67}$. The most common agent injected intravitreally is melphalan and, thus far, results have shown success in treating vitreous seeding ${ }^{68}$. However, as with other methods of delivery, there are known ocular toxicities associated with intravitreal administration of chemotherapeutics ${ }^{69}$. Taken together, it is clear chemotherapy maintains a role in the treatment of $\mathrm{Rb}$.

\section{Enucleation}

The practice of enucleation, or removal of the eye(s) for management of $\mathrm{Rb}$ dates back to the $19^{\text {th }}$ century and is still one of the most frequently used treatments. As the first goal of managing $\mathrm{Rb}$ is saving the patient's life, those with advanced intraocular, unilateral disease often undergo primary enucleation. For those patients with advanced bilateral $\mathrm{Rb}$, the physician will examine both eyes, make a judgement on which eye is likely more salvageable, and the worse eye may be enucleated with other treatment (i.e. IVC, IAC, cryotherapy, thermotherapy) being administered to the more salvageable eye. And, while offering a "cure" for Rb, enucleation is a still a less than desirable treatment as it leaves patients permanently scarred, partially (unilateral) or fully (bilateral) blind, and may have negative psychological side effects on those undergoing the procedure $\mathrm{p}^{70-72}$. 


\section{Immune ablation and autologous stem cell therapy (ASCT)}

Some small case reports have illustrated the success of high dose chemotherapy followed by autologous stem cell transplantation (ASCT) ${ }^{73-77}$. In these cases, patients afflicted with extraocular disease or those with metastatic disease lacking CNS involvement are treated with high dose chemotherapy, radiation, and followed by autologous stem cell therapy. This therapy permits the use of very high doses of chemotherapy, radiation, or both and for the immune system to be reconstituted over time using the patients' own stem cells. ASCT has proven more successful for patients presenting with metastatic disease than chemotherapy alone with approximately $73 \%$ of patients receiving chemotherapy alone developing relapse vs $47 \%$ of patients receiving chemotherapy and $\mathrm{ASCT}^{73,78}$.

\section{Models of Retinoblastoma}

\section{In vitro models}

Within the field of $\mathrm{Rb}$ research, there are three well-characterized human cell lines: Y79, Weri-Rb1, and RB355. The first Rb cell line, Y79, was derived from a 2.5 year old Caucasian female with advanced intraocular and metastatic disease ${ }^{79}$. Weri-Rb1 was isolated from a 1 year old Caucasian female with no family history of the disease and lack of metastasis ${ }^{80}$. Similarly, RB355 was isolated from the eye of a child with no family history of the disease ${ }^{81}$. As neither of these cells were clonally expanded, these cells are heterogeneous and further work soon has discovered RB355 is genetically related to Y79 and is considered a subclone ${ }^{82}$. Y79 is used to study more aggressive, invasive $\mathrm{Rb}$ and represents the metastatic form of the disease and Weri-Rb1 the less invasive, nonmetastatic model for $\mathrm{Rb}$.

\section{In vivo models}

LH $\beta$-Tag model. One of the earliest in vivo $\mathrm{Rb}$ models was created by accident. While attempting to induce expression of both SV40 large $\mathrm{T}$ and small $\mathrm{t}$ antigens in the pituitary (using the luteinizing hormone beta subunit) to create a model for pituitary tumors, it was found that a mouse (and its direct offspring) formed bilateral disease ${ }^{83}$. Later work elucidated that the large $T$ antigen was capable of disrupting normal $\mathrm{pRb}$ tumor suppressor function (as well as p53), thus giving rise to tumors ${ }^{84}$.

Genetic knock-out and knock-in models. The loss of $R B 1$ was thought to underlie the formation of $\mathrm{Rb}$ and, although still true in most cases, does not induce malignant tumor development in mice. Genetic models with defective $R B 1$ do not form $\mathrm{Rb}$, instead form benign tumors called retinomas ${ }^{23}$. Following this revelation, work showed $R B 1^{-/} / p 107^{-/}$mice formed $\mathrm{Rb}$ tumors, demonstrating $\mathrm{Rb}$ tumor formation is not specific to just $R B 1$ mutations and p107, another member of the pocket protein family, 
could suppress $\mathrm{Rb}$ tumor formation. Yet this model failed to replicate Rb tumorigenesis ${ }^{85}$. Similarly, $R B 1^{-/-} / p 130^{-/-}$double knock-out (DKO) mice showed lesions consistent with $\mathrm{Rb}$ (and similar to $R B 1 / p 107 \mathrm{DKO}$ mice) ${ }^{86}$. The tumors in $R B 1 / p 130 \mathrm{DKO}$ mice form earlier than those in the $R B 1 / p 107 \mathrm{DKO}$ mice. As $\mathrm{Rb}$ is known to have intact, normal p53 expression, the question still remained why $\mathrm{p} 53$ could not prevent $\mathrm{Rb}$ tumor formation. Novel work soon identified $M D M X$ in playing a key role in tumor formation by inactivating the p53 tumor suppressor pathway ${ }^{24}$. Still more complex models have been generated encompassing more knockout combinations to further examine the genetics of $\mathrm{Rb}$. A table of these models can be seen in Figure 1-1 ${ }^{87}$. More work has gone on to show these murine models and human $\mathrm{Rb}$ samples are molecularly similar ${ }^{88}$.

Xenografts. The first $\mathrm{Rb}$ xenograft (human $\mathrm{Rb}$ cells or tissue surgically grafted into mice) used was over 40 years ago, in which the two human Rb cell lines, Y79 and Weri-Rb1 were independently injected into the anterior chamber of rabbits to measure their abilities to form tumors ${ }^{80}$. Additional xenografts systems have been made by inoculating cells into the flanks of immunocompromised nude mice ${ }^{89}$. The limitations of the flank xenograft model are apparent as the challenge is treating $\mathrm{Rb}$ in its native environment. Further xenograft models implemented intravitreal inoculation of human cells into the eyes of both mice ${ }^{37}$ and rats ${ }^{90}$. Subretinal cell inoculations have also been utilized to make orthotopic xenografts and more closely mimic the pathophysiology of $\mathrm{Rb}$. Orthotopic xenografts have been made using multiple mammals, including mice ${ }^{91}$ as well as rabbits ${ }^{92}$. Finally, fresh tissue harvested from a patient was transplanted into nude mice, however, this was done subcutaneously ${ }^{93}$. The culmination of these previous models ultimately led to the establishment of an orthotopic patient-derived xenograft model (PDX) ${ }^{27}$, a novel system where tissue or cells from a patient's Rb tumor are implanted into an immunodeficient mouse to create a similar environment to the native tumor.

\section{Challenges in the Retinoblastoma Field}

The greatest challenge in the $\mathrm{Rb}$ field is the presence of small pockets of tumors floating in the vitreous cavity, called vitreous seeds (or seeding). Regardless of classification scheme, ocular salvage is "very unfavorable" in eyes with extensive vitreous seeding ${ }^{6}$. Often times, recurrence of disease is attributed to vitreous seeds. Traditionally, EBRT was used to combat and manage the presence of these seeds. However, EBRT exposes patients to elevated risks for secondary malignancies and, historically, ocular salvage rates rarely exceed $50 \%{ }^{94}$. IVC was then used to target seeds, but given their location within the vitreous, cytotoxic concentrations of chemotherapies rarely achieved ${ }^{95}$. Ocular salvage rates are approximately $50 \%$ with IVC $^{94}$. After the introduction of IAC, preliminary reports claimed ocular salvage rates around $70 \%{ }^{96}$ although, there exist conflicting reports (with longer follow up) of ocular salvage rates around $50 \%{ }^{97}$. Outcomes are similar to that of systemic therapy (IVC) as selected agent(s) did not readily permeate the BRB into the vitreous and thus, were unable to achieve cytotoxic concentrations. IViC was utilized and initial ocular survival rates 


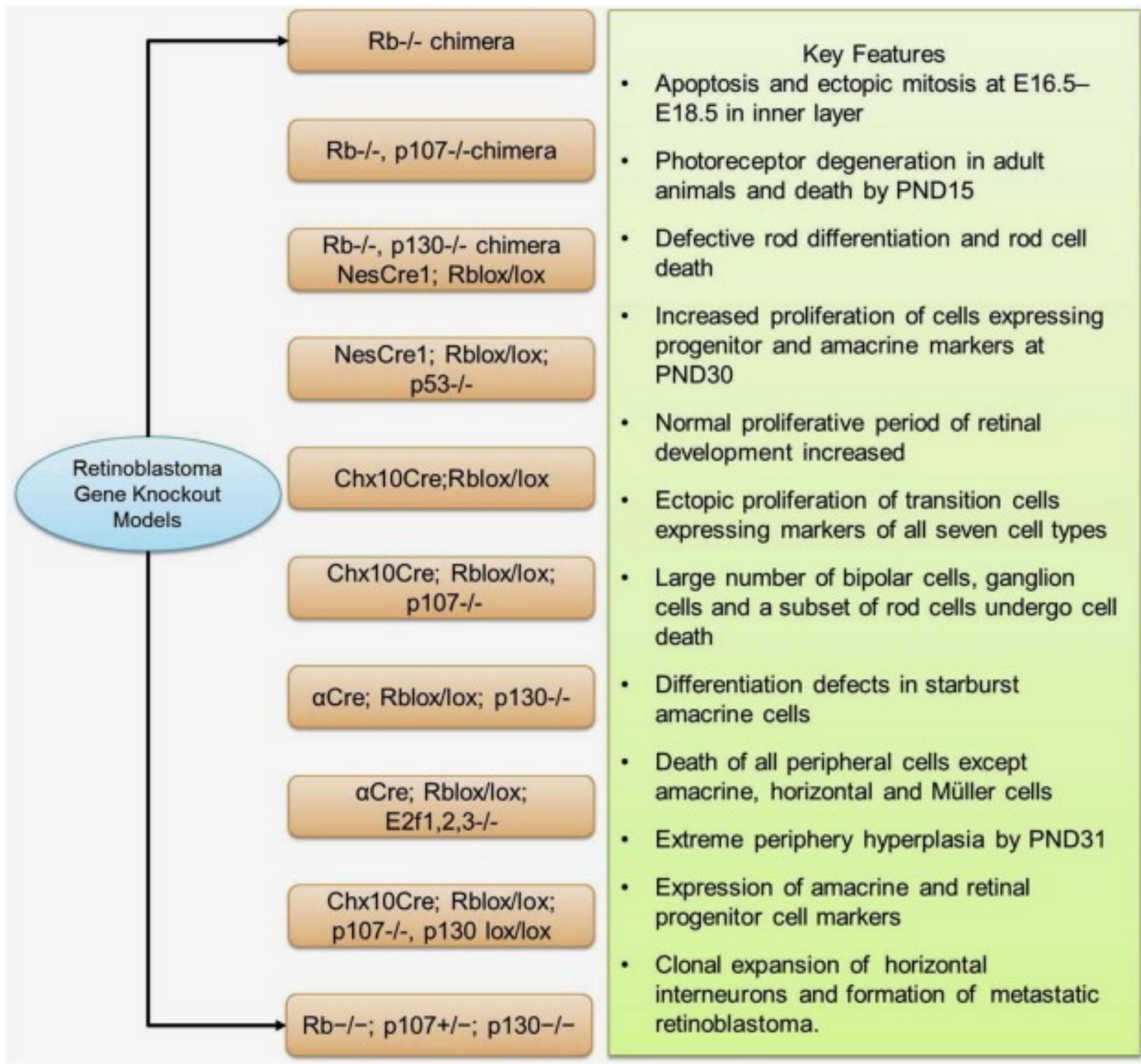

Figure 1-1. Genetic knockout models of retinoblastoma.

There are multiple rodent models of retinoblastoma $(\mathrm{Rb})$. Unlike that seen in the human disease, loss of $R B 1$ heterozygosity in mice does not result in tumor formation and $R B 1$ knockout is embryonically lethal. Therefore, using Cre-Lox technology was used to generate retina-specific knockdowns. These models further illustrate how $\mathrm{Rb}$ is a very heterogenic tumor with few mutations capable of initiating tumorigenesis. Other transgenic models include viral-oncogene induced models (LH $\beta$-Tag) and non-transgenic models, including xenografts, orthotopic xenografts, and patient-derived orthotopic xenografts. Reprinted with permission from Saudi J Ophthalmol, 27 (3), Nair, RM. et al. Animal models in retinoblastoma research, p141-146, Copyright (2013), with permission from Elsevier. 
increased up to $84 \%{ }^{98}$. Despite a number of ocular oncologists' best efforts, long-term ocular salvage rates for those patients treated with IViC have remained at $70 \%{ }^{99}$.

Subconjunctival administration of carboplatin has also been tested and ocular survival rates were $74 \%{ }^{67}$.

An explanation of why vitreous seeding remains so difficult to treat is the lack of preclinical modeling. While there are ample models of $\mathrm{Rb}$ to study tumor development and drug efficacy, there is an inadequacy of models that develop vitreous seeding. Currently, the only in vivo model capable of forming vitreous seeds is the orthotopic xenograft rabbit model ${ }^{92}$. As a result of this, the biology of vitreous seeding remains rather elusive. Only recently have studies begun to elucidate more about vitreous seeding and how it translates clinically ${ }^{100,101}$.

Another reason why ocular salvage rates have not improved is the absence of targeted therapies. Most chemotherapies used to treat $\mathrm{Rb}$ are broadly targeting cells undergoing rapid division; however, these seeds are largely necrotic and show reduced proliferation $^{100}$. To address this, disrupting molecular targets, such as spleen tyrosine kinase (SYK), histone deacetylase (HDAC), and MDM2/X/4, have been tested in vitro and in vivo using various small molecule inhibitors and are yet to make it into a clinical setting ${ }^{24,27,102}$. Most of these challenges, though, are a direct result of a deficiency in understanding the vitreous as a microenvironment. The hope is to develop and utilize new therapeutics to assist in clearing these tumors and improve ocular salvage rates beyond the current $70 \%$ threshold, but this can only be accomplished by growing our understanding of vitreous seeds and their unique biology.

\section{The Retinoblastoma Tumor Microenvironment}

The idea that tumors are not independent entities and that they interact with other systems in the body is not new. Stephen Paget, in 1889, published work examining the metastatic patterns of post mortem breast cancer patients and concluded this could not be by chance; the cancer and the tissue it metastasized to played a role in development of metastatic disease ${ }^{103}$. Over a century later, it is now well known that tumors are not independent entities. Instead, the compartment in which tumors grow and survive is called the "tumor microenvironment" (TME). The TME encompasses tumor cells along with immune cells, endothelial cells, fibroblasts, cytokines, growth factors, enzymes, and other proteins ${ }^{104}$. And, while the TME is an integral part of tumor biology, very little is currently known about the Rb TME.

\section{The tumor microenvironment of endophytic and exophoytic Rb}

The two main growth patterns of primary $\mathrm{Rb}$ tumors are endophytic and exophytic (see: Primary Tumor Subtypes). Tumors exhibiting both endophytic and exophytic growth are often highly vascularized, illustrating the abundance of endothelial cells and pericytes ${ }^{5}$. Using irradiated, transgenic mice and transplanting them with GFP- 
labeled bone marrow cells, it was discovered that there were a high percentage of tumorassociated macrophages (TAMs) in advanced intraocular $\mathrm{Rb}$ and these TAMs were localized largely to the vasculature of the tumor ${ }^{105}$. How these TAMs interact with the tumor is still poorly understood but these TAMs may promote primary tumor vasculature and suppress immune function while simultaneously secreting growth factors and chemokines that drive processes such as survival and resistance.

Despite primary tumors having sufficient blood supply, seeds (be they vitreal or subretinal) lack this same blood supply and are known to be avascular ${ }^{5}$. If these cells lack vasculature, they are unable to receive normoxic (normal oxygen, $\mathrm{O}_{2}$ ) levels and grow in a low $\mathrm{O}_{2}$, or hypoxic (low $\mathrm{O}_{2}$ concentrations), environment. $\mathrm{Rb}$ cells grown in a hypoxic environment undergo alterations in their metabolism to survive in this avascular system and divided at a much lower rate ${ }^{106}$. Furthermore, failure of radiotherapy or chemotherapy is often attributed to hypoxic status ${ }^{107}$.

\section{The vitreous as a microenvironment}

The vitreous (sometimes referred to as vitreous body or vitreous humor) is a translucent medium that encompasses approximately $80 \%$ of the eye. Composed of $99 \%$ water, the remaining $1 \%$ is a solid phase composed of sugars, salts, collagen, and hyaluronan, as well as chemokines, cytokines, and growth factors ${ }^{108-111}$. These chemokines, cytokines, and growth factors are important in homeostasis but are often sequestered in collagen ${ }^{112}$. These molecules, normally present in the vitreous, are also known to be associated with and exacerbate various ocular pathologies, most notably diabetic retinopathy (DR) and proliferative vitreoretinopathy (PVR) in rhegmatogenous retinal detachments ${ }^{113,114}$. However, very little is currently known about interactions between tumor cells and the vitreous. Furthermore, few studies have attempted to characterize the vitreous as a tumor microenvironment and elucidate the role it plays in vitreous seeding. Recent reports began classifying patterns of vitreous seeding as one of three types: 1) "dust," 2) "spheres," and 3) "clouds"100,101. Briefly, histopathology studies revealed "dust" seeds are composed primarily of individual, viable cells while "clouds" were primarily composed of necrotic $(>90 \%)$ cells with few viable cells; finally, those classified as "spheres" are viable cells with necrotic cores and are thought to represent the most aggressive seed phenotype ${ }^{101}$. Interestingly, the presence of macrophages was demonstrated in all subtypes of vitreous seeds ${ }^{101}$. The role(s) of macrophages has yet to be elucidated. Furthermore, how seed classification relates to potential treatment options and long-term outcomes have yet to be investigated. Therefore, the vitreous may be considered a reservoir of untapped potential that may be advantageous for tumor cells to gain access to but also to target as potential therapeutic avenues. Rb cells invading the vitreous may have access to all of these growth factors, cytokines, and chemokines that may aid in tumor survival. 


\section{The extracellular matrix}

One area that has begun to be studied in $\mathrm{Rb}$ is the extracellular matrix (ECM) and how tumor cells interact with the ECM. The ECM provides physical support and is involved in healthy cell division, migration, differentiation, and survival. Composed primarily of proteoglycans and fibrous proteins (e.g. collagen, fibronectin, laminin, etc.), the ECM is important not only in homeostasis, but also wound healing and disease ${ }^{115}$. One of the most well characterized interactions is that of matrix metalloproteinases (MMPs) and the ECM. MMPs are a family of over 20 proteolytic enzymes with roles in tissue remodeling, wound healing, angiogenesis, inflammation, and tumor metastasis (Figure 1-2). Their roles in the eye as well as tumor biology will be discussed below.

Matrix metalloproteinases in the healthy and diseased eye. One of the basic functions of MMPs is tissue maintenance and remodeling ${ }^{116}$. In the normal eye, MMPs are expressed by a number of different cell types including fibroblasts, monocytes, macrophages, leukocytes, and endothelial cells, all of which are important in homeostasis. MMPs are expressed at baseline levels within both anterior and posterior segments of the eye and are transcriptionally regulated by specific tissue inhibitors of matrix metalloproteinases (TIMPs); it is only under stress (such as wound healing) that they may be expressed and activated ${ }^{117-119}$. One of the classic models for MMP activity is corneal wounds where the corneal epithelium is damaged and experiences inflammation and regeneration ${ }^{120}$. Moreover, as MMPs are expressed by endothelial cells (and other inflammatory cells), they play a vital role in both healthy neovascularization as well as those attributed to disease such as $\mathrm{DR}^{121}$ and $\mathrm{AMD}^{122}$. Studies have begun to elucidate the role MMPs secreted by cells within the trabecular meshwork play in the flow of aqueous humor and how these molecules may be exploited to reduce intraocular pressure for management of glaucoma ${ }^{123}$. Furthermore, published reports have discovered the presence of MMPs within healthy vitreous ${ }^{124}$. However most of these enzymes were found to be pro-MMPs (the inactive form of the enzyme) ${ }^{124}$. The mechanism and necessity of MMPs are well understood yet their role(s) in the eye has yet to be unraveled - it is known they play a role in a number of ocular pathologies but their (clinical) therapeutic value is uncertain at this time. One role that is clearer is the role MMPs play in cancer.

Matrix metalloproteinases in cancer. As mentioned above, tumors do not act alone; alternatively, they interact with a variety of different proteins and cell types, all that culminate in a malignant tumor. One such relationship is that of tumor cells and the ECM, more specifically that related to the most abundant protein in the body and the ECM: collagen. The MMP-collagen interface is well understood under normal, physiological conditions to be vital for proper development and maintenance. However, some cancers are known to overexpress MMPs, yet most malignancies do not and, instead depend on normal, heathy cells to synthesize these enzymes for them through interleukins, interferons, and other cytokines and chemokines ${ }^{125}$. With these overactive enzymes, tumors are able to utilize MMP functions and induce a number of the hallmarks of cancer including angiogenesis, immune evasion, and survival ${ }^{104,126}$. With this 


\begin{tabular}{|c|c|c|}
\hline MMP designation" & Structural class & Common name(s) \\
\hline MMP-1 & Simple hemopexin domain & $\begin{array}{l}\text { Collagenase-1, interstitial collagenase, fibroblast collagenase, } \\
\text { tissue collagenase }\end{array}$ \\
\hline MMP-2 & Gelatin-binding & $\begin{array}{l}\text { Gelatinase A, } 72-k D a \text { gelatinase, } 72-k D a \text { type IV collagenase, } \\
\text { neutrophil gelatinase }\end{array}$ \\
\hline MMP-3 & Simple hemopexin domain & $\begin{array}{l}\text { Stromelysin-1, transin-1, proteoglycanase, procollagenase- } \\
\text { activating protein }\end{array}$ \\
\hline MMP-7 & Minimal domain & Matrilysin, matrin, PUMP1, small uterine metalloproteinase \\
\hline MMP-8 & Simple hemopexin domain & $\begin{array}{l}\text { Collagenase-2, neutrophil collagenase, PMN collagenase, } \\
\text { granulocyte collagenase }\end{array}$ \\
\hline MMP-9 & Gelatin-binding & Gelatinase B, 92-kDa gelatinase, 92-kDa type IV collagenase \\
\hline MMP-10 & Simple hemopexin domain & Stromelysin-2, transin-2 \\
\hline MMP-11 & Furin-activated and secreted & Stromelysin-3 \\
\hline MMP-12 & Simple hemopexin domain & $\begin{array}{l}\text { Metalloelastase, macrophage elastase, macrophage } \\
\text { metalloelastase }\end{array}$ \\
\hline MMP-13 & Simple hemopexin domain & Collagenase- 3 \\
\hline MMP-14 & Transmembrane & MT1-MMP, MT-MMP1 \\
\hline MMP-15 & Transmembrane & MT2-MMP, MT-MMP2 \\
\hline MMP-16 & Transmembrane & MT3-MMP, MT-MMP3 \\
\hline MMP-17 & GPI-linked & MT4-MMP, MT-MMP4 \\
\hline MMP-18 & Simple hemopexin domain & Collagenase-4 (Xenopus; no human homologue known) \\
\hline MMP-19 & Simple hemopexin domain & RASI-1, MMP-18 \\
\hline MMP-20 & Simple hemopexin domain & Enamelysin \\
\hline$M M P-21^{5}$ & Vitronectin-like insert & Homologue of Xenopus XMMP \\
\hline MMP-22 & Simple hemopexin domain & CMMP (chicken; no hurnan hormologue known) \\
\hline MMP-23 & Type II transmembranel & $\begin{array}{l}\text { Cysteine array MMP (CA-MMP), femalysin, MIFR, } \\
\text { MMP-21/MMP-22" }\end{array}$ \\
\hline MMP-24 & Transmembrane & MT5-MMP, MT-MMP5 \\
\hline MMP-25 & GPI-linked & MT6-MMP, MT-MMP6, leukolysin \\
\hline MMP-26 & Minimal domain & Endometase, matrilysin-2 \\
\hline $\mathrm{MMP}-27^{ \pm}$ & Simple hemopexin domain & \\
\hline MMP-28 & Furin-activated and secreted & Epilysin \\
\hline No designation & Simple hemopexin domain & Mcol-A (Mouse) \\
\hline No designation & Simple hemopexin domain & Mcol-B (Mouse) \\
\hline No designation & Gelatin-binding & 75-kDa gelatinase (chicken) \\
\hline \multicolumn{3}{|c|}{ 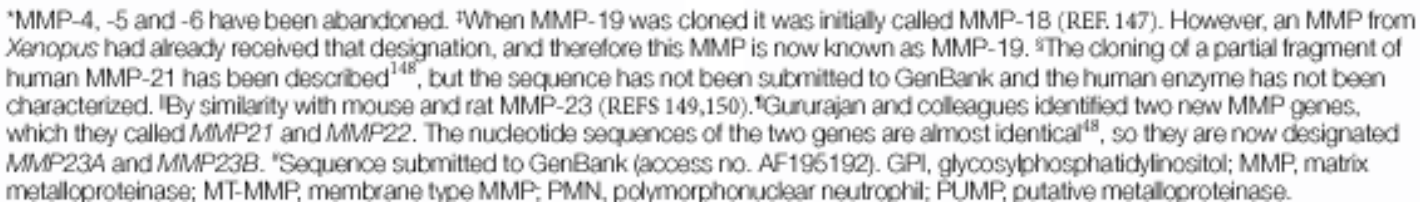 } \\
\hline
\end{tabular}

Figure 1-2. Members of the Matrix Metalloproteinase (MMP) Family.

The matrix metalloproteinase family of protease enzymes includes over 20 members. These enzymes are vital for normal development and tissue organization. These molecules are also involved in a number of pathological processes including angiogenesis, wound healing, and cancer. Most commonly, MMP-1, -2, and -9 are associated with cancer progression and metastasis. Reprinted with permission from Nat. Rev. Cancer Egeblad M., Werb Z. New functions for the matrix metalloproteinases in cancer progression. , 2: 163-176, (2002), with permission from Springer Nature. 
knowledge, it is quite understandable why patient prognosis (in a number of malignancies) is significantly worse when high levels of MMPs are detected ${ }^{127,128}$. Moreover, patient prognosis is ultimately worse due to higher risk for metastasis, the primary cause for cancer-associated mortalities, in which MMPs play a decisive role ${ }^{129}$. The epithelial-to-mesenchymal transition (EMT) is classically understood to be the initiation of metastatic disease. Briefly, cells undergoing EMT lose their cellular adhesion (i.e. tight junctions), show an increase in motility (via cytoskeletal remodeling), and acquire invasive potential through increases in MMPs, most commonly those of MMP-1, MMP-2, and MMP- $9^{130}$. Furthermore, EMT has been demonstrated to confer resistance of cancer cells to chemotherapy ${ }^{131}$.

While there are a number of chemical inhibitors of MMPs, the first "successful" inhibitor was BB-94 (aka Batimastat) and was the first broad-spectrum MMP inhibitor (MMPI) to reach clinical trials ${ }^{132}$. Used as an anti-angiogenic and anti-metastatic therapy, Batimastat was promptly abandoned due to an inability to be administered orally ${ }^{133}$. Perhaps the most notable MMP inhibitor, BB-2516 (sold by British Biotech under the name Marimastat), was a non-specific MMPI marketed as a new antineoplastic and oncostatic drug for stomach cancer (and was capable of being administered orally). Both preclinical and phase I clinical trials were conducted and showed reduced tumor burden (in xenografts) and higher rates of two-year survival rates (in patients) ${ }^{134,135}$. Ultimately, Marimastat failed in phase III clinical trials ${ }^{126}$. As most of the early MMPIs were nonspecific, side effects such as arthritis and musculoskeletal pain were problematic ${ }^{126}$. In response to toxicities, the next generation of therapeutics are being designed to be MMPspecific. New technologies such as antibodies and antisense oligonucleotides are currently being developed and investigated but clinical trials using these materials are lacking $^{126}$.

However, when considering the vitreous as a microenvironment, it is the lesserknown role of MMPs that is perhaps most interesting. With the vitreous containing ECM molecules (such as collagen), many of the proteins within the vitreous are sequestered in the ECM; it is not until this ECM undergoes proteolysis these growth factors are free to bind their receptors and are considered bioactive ${ }^{112}$. Some of these same protein families are strongly linked to the hallmarks of cancer ${ }^{104}$. One such family, the platelet-derived growth factor (PDGF) family is known to play roles in both ocular pathologies and cancer.

\section{The Platelet-Derived Growth Factor Family}

\section{Introduction}

There are currently nine genetically-identified members of the platelet-derived growth factor (PDGF) family that include: two classic PDGF family members: PDGF-A and PDGF-B, two novel family members: PDGF-C and PDGF-D; as well as the vascular endothelial growth factor (VEGF) family: VEGF-A, VEGF-B, VEGF-C, VEGF-D, and 
placental growth factor (PLGF). There are three receptor tyrosine kinases (RTKs) for the PDGF family including two homodimers: PDGFR $\alpha$ and PDGFR $\beta$, as well as a heterodimer: PDGFR $\alpha \beta$. These proteins form homodimers (i.e. PDGF-AA, PDGF-BB, etc.) and, to date, only one heterodimer has been found, PDGF-AB. As a part of their homology, these signaling molecules have different affinities for their receptors (Figure 1-3). From early gastrulation and development to hematopoiesis and wound healing, this family of proteins is vital to normal health and growth ${ }^{136}$. Mice lacking copies of either $P D G F A$ and $P D G F B$ or either receptor (PDGFRA or $P D G F R B)$ are embryonically lethal ${ }^{137-139}$. While little is currently known about the roles of PDGF-C and PDGF-D in physiology, work has shown $P D G F C$ deficiency may be perinatally lethal ${ }^{140}$ but this appears to be background strain- (and sex-) dependent ${ }^{141}$. Interestingly, PDGFD knockouts are quite viable with a slightly abnormal vascularized phenotype ${ }^{142}$. Furthermore, some attempts at identifying tissue-specific expression of PDGFs have elicited that PDGF-A and PDGF-C are most commonly affiliated with epithelial cells and neuronal progenitor cells, PDGF-B is expressed by endothelial cells and mature neurons, and, while less clear, PDGF-D on smooth muscle cells and fibroblasts ${ }^{143}$. Still, these patterns are generalized and fail to capture the complex nature of these signaling molecules. However, given their broad, overarching roles in physiology, it is no surprise these molecules have been implicated in disease pathologies as well. The roles members of the PDGF family play in both ophthalmology and cancer biology are discussed further below.

\section{Platelet-derived growth factors in ocular health and disease}

While some work has demonstrated PDGFs (specifically PDGF-BB) mediated corneal wound healing ${ }^{120}$, initial investigations of the PDGF family in ocular health focused primarily on retina and development due to prior studies identifying the PDGF family as requirements for proper development and organization of the central nervous system (CNS). PDGF-AA ligand and its primary receptor, PDGFR $\alpha$, were shown to be involved in retinal development primarily in the retinal pigment epithelium and astrocytes while PDGF-BB ligand and its primary receptor, PDGFR $\beta$, were detected in the hyaloid canal and in vasculature ${ }^{144,145}$. Other work incidentally discovered that when $P D G F R B$ is disrupted in the retina, a lack of pericytes leads to dysregulated vasculature and proliferative retinopathy ${ }^{146}$. Further, expression of $P D G F B$ in photoreceptors of mice demonstrated rampant neovascularization and retinal detachments ${ }^{147}$. These studies inadvertently shaped how these signaling molecules would be viewed in the context of the eye: PDGF-AA as a molecule involved in the development of the eye and PDGF-BB as vasculature-specific molecule ${ }^{148}$.

Given their role in neovascularization, more translational and clinical studies began examining the PDGF family in patients in diseases that feature neovascularization as a primary etiology. High levels of PDGF-AB were detected in patients with retinopathies in one of the pioneering studies of PDGFs in ophthalmology ${ }^{149}$. These levels were increased in patients with both diabetic and non-diabetic retinopathies, 


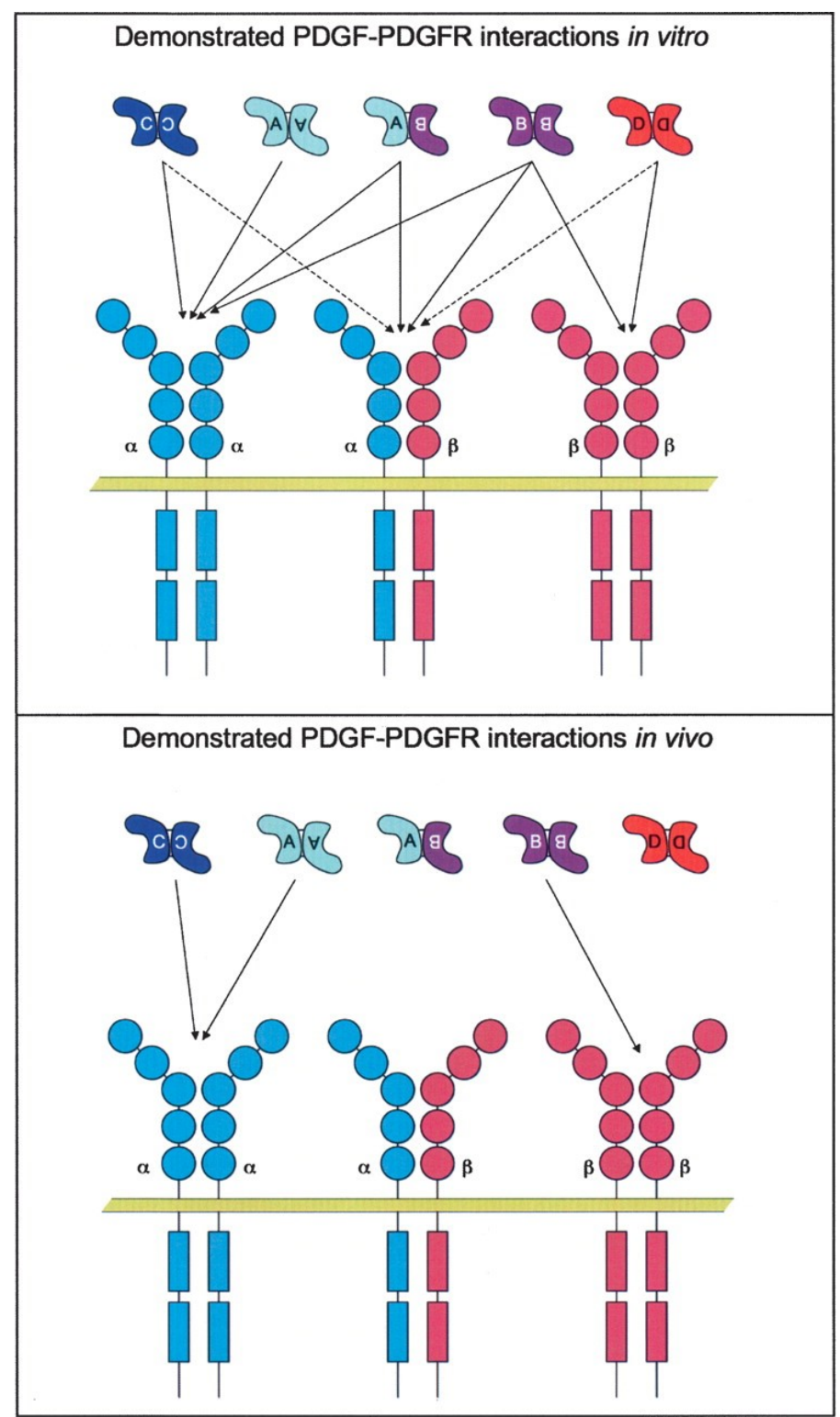

Figure 1-3. Members of the PDGF family and their receptors.

Platelet-derived growth factors (and their receptors) play important roles in both health and disease. PDGF-AA and PDGF-CC have high affinities for PDGFR $\alpha$; PDGF-BB has a high affinity for PDGFR $\beta$ and PDGF-DD is thought to as well. PDGF-AB has the highest affinity for its corresponding heterodimer receptor PDGFR $\alpha \beta$. There exist many discrepancies between ligand-receptor interactions in vitro (top panel) and in vivo (bottom panel). But given their homology, it is thought most ligands are capable of binding both homodimeric and heterodimeric receptors. Reprinted with permission from Genes Dev, 22 (10), Andrae, J., et al. Role of platelet-derived growth factors in physiology and medicine. p1276-1312, Copyright (May 15, 2008), with permission through Creative Common CC-BY. 
illustrating glucose-independent regulation of PDGFs, inverse to what had been previously suggested ${ }^{150}$. More studies added to these data, correlating PDGF levels with disease progression and severity ${ }^{151,152}$.These studies helped identify a new therapeutic target and pre-clinical studies examined the potential value of these targets. Targeting PDGFs, specifically PDGF-BB, was shown to be successful in preventing neovascularization pre-clinically, and clinical trials followed shortly thereafter ${ }^{153,154}$. However, these clinical trials ultimately failed to meet outcomes and the trials were terminated $^{155}$. The future of PDGF's therapeutic value in remedying ocular diseases is uncertain but one area where anti-PDGF/PDGFR therapy has shown tremendous value is in oncology.

\section{Platelet-derived growth factors in cancer}

PDGFs are known to promote tumor angiogenesis; without proper blood supply, tumors will fail to grow ${ }^{156}$. However, the main function of PDGFs is that of a mitogen, or a molecule capable of inducing cell division. This signaling also has the potential to be an autocrine loop and was seen as potentially useful in tumorigenesis ${ }^{157}$ Though mutations in members of the PDGF family are somewhat rare, this autocrine loop may provide selective growth advantages for cells that overexpress a receptor (or receptors) and may produce higher levels of the ligand(s $)^{158}$. One of the most notable examples of tumors with overexpression / amplification of PDGFs is glioblastoma, which features an increase in PDGFRA with higher expression correlating to worse prognosis ${ }^{159}$. There have multiple attempts of using tyrosine kinase inhibitors (TKIs) to treat glioblastoma, but results have been disappointing ${ }^{160}$.

There are tumor types that are responsive to TKIs targeting PDGFRs. One such tumor is that of gastrointestinal stroma (GISTs). GIST tumors previously carried abysmal prognoses with survival rates less than one year; however, work identified these tumors frequently possess mutations in either KIT or PDGFRA and may be targeted using TKIs 161. Patients benefited quite strongly to treatment with imatinib mesylate (IM, a known inhibitor of c-kit as well as PDGFRs) ${ }^{161}$.

Another tumor, dermatofibrosarcoma protuberans (DP), frequently harbor translocations of the $P D G F B$ whereby the gene is fused with that of the gene encoding for collagen type 1 alpha 1 (COL1A1) and results in excessive PDGF-B production ${ }^{162}$. This production establishes an autocrine loop, promoting rapid cell proliferation and resulting in tumorigenesis. The same TKI, imatinib mesylate, was utilized to block PDGF-BB/PDGFR $\beta$ signaling and has been approved for administration in those afflicted with $\mathrm{DP}^{163}$. There are ongoing investigations into the PDGF family and their roles in other malignancies as well, including leukemias as well as prostate, lung, breast, and liver cancers. And though recent work has cast a shadow of doubt on anti-PDGF/PDGFR therapy in the field of ophthalmology for neovascularization, oncologists and cancer patients have vastly benefited from the therapeutic strategies targeting these key molecules. 


\section{Vascular endothelial growth factor, a sub-family of platelet-derived growth factor family}

The other members of the PDGF family, vascular endothelial growth factors (VEGF), are more well-understood than the PDGFs. Similar to the PDGF family though, VEGFs bind receptor tyrosine kinases (RTKs) and are capable of inducing downstream signaling. The VEGF family is most noted for the key role it plays in promoting endothelial cell migration and division and therefore, to angiogenesis ${ }^{164}$. As discussed previously, angiogenesis is necessary for tumor growth ${ }^{156}$. Therefore, by removing access to blood supply from tumor cells, these cells may not divide and will die. This idea led to the development of the first anti-VEGF monoclonal antibody, bevacizumab, which was developed and utilized (alongside chemotherapy) for treatment of metastatic colorectal cancers. Today, bevacizumab has been extensively studied in a number of malignancies and is even approved for treatment of metastatic colon, renal, and breast cancers, as well as use in glioblastomas, lung cancers, and ovarian cancers ${ }^{165}$. Bevacizumab has even been tried preclinically for treatment of $\mathrm{Rb}$ in xenografts ${ }^{166,167}$. Despite modest success, anti-VEGF therapy has failed to penetrate a clinical setting for the treatment of $\mathrm{Rb}$. Moreover, the focus in $\mathrm{Rb}$ that has the largest unmet need is in treating vitreous seeding and, as these seeds are avascular, anti-angiogenic therapy is hardly appropriate.

The area that has heralded the success of anti-VEGF therapy the most is in ophthalmology. While studies were being conducted on the role VEGF may play on tumor angiogenesis, other studies elucidated that VEGF played a similar role in other ocular pathologies, namely AMD and $\mathrm{DR}^{168}$. Just two years after approval of bevacizumab for metastatic colon cancer, a more diffusible version of bevacizumab, ranibizumab, was approved by the Federal Drug Administration (FDA) for treatment of $\mathrm{AMD}^{168}$. While successful, anti-VEGF therapy does not work for all patients and only about one-third of patients receive improved visual acuity over time ${ }^{169}$. To combat this problem, anti-VEGF and anti-PDGF therapies were utilized together. These clinical trials examined anti-VEGF therapy and compared it to a combined anti-VEGF/-PDGF therapy. The combination therapy failed to increase visual acuity when compared to anti-VEGF therapy alone and, thus, anti-PDGF therapies were abandoned ${ }^{155}$.

\section{Rationale and Specific Aims}

It is clear that great strides have been made in treating $\mathrm{Rb}$. Survival rates are greater than $95 \%$ in developed countries. However, vitreous seeding remains a clinical challenge in treating a patient with $\mathrm{Rb}$. Numerous studies have shown the importance of the tumor microenvironment (TME) in promoting tumorigenesis, survival, metastasis, and resistance to therapies, yet little is known about the $\mathrm{Rb} T \mathrm{TME}$ and how the tumor interacts with its TME.

The extracellular matrix (ECM) is of vast importance in the TME, as the tumor cells are capable of utilizing the ECM to generate new blood supply, increase pro-tumoral 
inflammation, and utilize proteolytic processing MMPs to gain access to an increased supply of growth factors. One such family of growth factors, the PDGF family, has been well studied in both vitreoretinal pathologies as well as multiple malignancies. The central hypothesis of this study is that the interactions between the $\mathrm{Rb}$ tumor and its microenvironment are permissive for the growth and sustain of vitreous seeds. To address the central hypothesis I utilized basic Rb cell lines, murine $\mathrm{Rb}$ xenografts samples, patient-derived xenograft samples, and ex vivo human samples from healthy donors and $\mathrm{Rb}$ patients. The central hypothesis of this study was tested by pursuing the following aims or objectives:

Aim 1: To test the prediction that MMPs within the ECM play a role in Rb tumor migration and angiogenesis by characterization of key pro-tumoral members of the MMP family in Rb cell lines. The role of these MMPs in migration and angiogenesis was investigated using pharmacological and genetic knock-down approaches. (Chapter 2)

Aim 2: To test the prediction that the PDGF-PDGFR $\beta$ signaling pathway plays a pivotal role in sustainment of $\mathrm{Rb}$. The abundance in the $\mathrm{Rb}$ vitreous and signaling activity of this pathway in an autocrine and paracrine (from the TME) manner makes it a candidate for targeted therapy for the management of vitreous seeds. (Chapter 3)

Aim 3: To test the pre-clinical safety of targeted anti-PDGFR $\beta$ therapy in other cellular components of the TME. The effects of targeted anti-PDGFR $\beta$ therapy in human retinal endothelial cells and the inherent properties of $\mathrm{Rb}$ in chemotherapy resistance. (Chapter 4) 


\section{CHAPTER 2. RESULTS I: INHIBITION OF MMP-2 AND MMP-9 DECREASE CELLULAR MIGRATION, AND ANGIOGENESIS IN IN VITRO MODELS OF RETINOBLASTOMA ${ }^{1}$}

\section{Introduction}

Retinoblastoma $(\mathrm{Rb})$ is the most common primary intraocular tumor in children with an incidence of approximately 12 cases per million children under 4 years of age in the United States ${ }^{171}$. Mutation of the tumor suppressor gene, $R B 1$, can lead to the disease sporadically or through inheritance. Germline mutations of $R B I$ account for approximately $40 \%$ of cases and exhibit an autosomal dominant pattern of inheritance ${ }^{172}$. Germline $R B 1$ often affects both eyes whereas the more common sporadic form of the disease is often unilateral and accounts for $60 \%$ of all cases ${ }^{172}$. If diagnosed early, intraocular $\mathrm{Rb}$ can be effectively treated; however, the more advanced disease can metastasize to the central nervous system (CNS) in which case, mortality rates are greatly increased ${ }^{173}$. Tumor invasion from the retina to the sclera and post laminar optic nerve are of high risk pathologic features for both hematogenous and CNS metastasis ${ }^{173}$. Clinical risk factors that increase the incidence of metastasis in these patients include older age ${ }^{174-176}$, laterality ${ }^{177}$, vascularity ${ }^{178,179}$, and stage present upon diagnosis ${ }^{180}$.

The dissemination of malignant neoplasms is assumed to require degradation of different components of the matrix and basement membrane. Matrix metalloproteinases (MMPs) are responsible for degradation of a number of extracellular matrix (ECM) components. There are over 20 recognized MMPs, each with specific substrate requirements and structural domains ${ }^{181-183}$. Among these are two highly associated with tumor dissemination and invasiveness ${ }^{184,185}$ : MMP-2 (aka gelatinase A) and MMP-9 (aka gelatinase B), which degrade type IV collagen and gelatin substrates. Cumulative work in different solid tumors has generated great interest in the development of MMP inhibitors (MMPI) as potential therapeutic anti-metastatic agents. Some synthetic MMPI have been tested in clinical trials in solid tumors other than $\mathrm{Rb}$ and show different levels of efficacy ${ }^{186,187}$. Recent clinical trials by Gilead Sciences are evaluating MMP activity in different solid tumors, including non-small cell lung carcinoma (NSCLC), pancreatic adenocarcinoma, colorectal cancer (CRC) and breast cancer, and their effects in the tumor microenvironment by using an anti-MMP-9 therapeutic antibody ${ }^{188}$. The antibody, GS-5745, is a humanized monoclonal antibody against MMP-9, which upon binding MMP-9 results in inhibition of ECM degradation and possibly a reduction in tumor growth and risk of metastasis ${ }^{189}$. Immunohistochemical analysis of primary $\mathrm{Rb}$ tumors show that MMP-2 and MMP-9 protein levels are higher in samples that had invaded the optic nerve ${ }^{190,191}$. To our knowledge, the effects of MMPI on Rb have not been analyzed

\footnotetext{
${ }^{1}$ Adapted with permission. Webb AH, Gao BT, Goldsmith ZK, et al. Inhibition of MMP-2 and MMP-9 decreases cellular migration, and angiogenesis in in vitro models of retinoblastoma. BMC Cancer. 2017;17(1):434. C BioMed Central.
} 
comprehensively in vitro. Here, we provide a detailed analysis of two MMPI on cellular viability, levels of pro-angiogenic factors, migration and immunomodulatory proteins in two well-studied Rb cell lines: Y79 and Weri-1. These two Rb cell lines have somewhat different characteristics, with Y79 exhibiting inherent metastatic properties and Weri-1 exhibiting non-metastatic properties. Our aim was to examine responses of both cell lines since it is likely that $\mathrm{Rb}$ tumors in vivo may contain mixed populations of tumor cells with varying metastatic potential. Our results demonstrate that pharmacological inhibition of MMPs reduces $\mathrm{Rb}$ cell viability, migration, and secretion of the pro-angiogenic factors VEGF and Angiopoietin-2 in either one or both types of $\mathrm{Rb}$ cell lines. These promising findings provide an impetus for future in vivo studies to evaluate MMPI as a potential adjunct therapy for $\mathrm{Rb}$ patients.

\section{Materials and Methods}

\section{Cell lines, growth media, and tissue culture conditions}

Y79 (ATCC-HTB-18) ${ }^{79}$, Weri-1 (ATCC-HTB-169) ${ }^{80}$, Retinoblastoma (Rb) tumor cell lines were purchased from the American Type Culture Collection (ATCC, Manassas, VA). Cells were grown in RPMI-1640 (MediaTech, Herndon, VA) supplemented with 20\% Fetal Bovine Serum (Hyclone, Logan, UT), 1\% of Penicillin G Sodium Salt/Streptomycin Sulfate (100X) (Lonza). Rb cell lines were grown under different conditions, including ARP100 (MMP-2 inhibitor, Santa Cruz Biotechnology) at $5 \mu \mathrm{M}$ and AG-L-66085 (MMP-9 inhibitor, Santa Cruz Biotechnology) at 5 $\mu \mathrm{M}$ concentration, unless otherwise specified. Incubation proceeded overnight at $37^{\circ} \mathrm{C} / 5 \% \mathrm{CO}_{2}$. The $\mathrm{IC}_{50}$ values for ARP100: MMP-2: 12nM; MMP-3: 4.5 $\mathrm{M}$; MMP-7: $50 \mu \mathrm{M}$. The $\mathrm{IC}_{50}$ values for AGL-66085: MMP-9: 5nM; MMP-1: 1.05 $\mu$ M.

\section{qPCR analyses}

RNA isolation. RNA from $2.5 \times 10^{6} \mathrm{Rb}$ cells was extracted following the Qiagen ${ }^{\circledR}$ miRNeasy Mini Kit (Qiagen, Valencia, CA) manufacturer's recommendations. $\mathrm{Rb}$ cells were harvested and centrifuged to remove all traces of cell culture medium. Cell pellets were lysed and homogenized prior to addition of chloroform. Samples were shaken vigorously for $15 \mathrm{~s}$ prior to centrifugation for $2 \mathrm{~min}$ at full speed. The upper colorless phase was transferred to a clean tube after centrifugation followed by a series of alcohol precipitations to provide ideal binding conditions. The lysate was loaded onto an RNeasy spin column with a silica membrane to bind the RNA and washed away contaminants. Residual DNA was removed by on-column DNase digestion. The concentrated RNA was eluted in $15 \mu \mathrm{L}$ of RNAse free water. RNA concentration was assessed by analysis on Nanodrop 2000 Spectophotometer (ThermoFisher Scientific) and purity was assessed by verifying absorbance ratios of 260nm and 280nm (A260/A280). Samples with absorbance ratios between 1.8 and 2.0 were considered free of contamination. 
cDNA synthesis and pre-amplification. Synthesis of cDNA was performed using the SuperScript ${ }^{\circledR}$ VILO ${ }^{\text {TM }}$ cDNA Synthesis Kit (Life Technologies, Grand Island, NY). Following manufacturer's directions, we used 100ng of isolated RNA (see section above) and combined them with $4 \mu \mathrm{L} 5 \mathrm{X}$ VILO Reaction Buffer and $2 \mu \mathrm{L} 10 \mathrm{X}$ SuperScript Enzyme Mix. Final volume per sample was $20 \mu \mathrm{L}$ using DEPC-treated water when necessary. Per manufacturer's directions, cDNA synthesis reaction was done at $25^{\circ} \mathrm{C}$ for $10 \mathrm{~min}$ followed by $42^{\circ} \mathrm{C}$ incubation for $60 \mathrm{~min}$ with reaction termination at $85^{\circ} \mathrm{C}$ for $5 \mathrm{~min}$. Material was pre-amplified using $10 \mu \mathrm{L} 2 \mathrm{X}$ TaqMan ${ }^{\circledR}$ PreAmp Master Mix, $5 \mu \mathrm{L}$ pooled $0.2 \mathrm{X}$ TaqMan ${ }^{\circledR}$ primers, and $5 \mu \mathrm{L}$ cDNA sample for a final volume of $20 \mu \mathrm{L}^{192}$. Preamplification reactions were conducted according to manufacturer's guidelines with a $10 \mathrm{~min}$ enzymatic activation step at $95^{\circ} \mathrm{C}$ followed by 14 cycles of denaturing $\left(95^{\circ} \mathrm{C}\right.$ for $\left.15 \mathrm{~s}\right)$ and annealing $\left(60^{\circ} \mathrm{C}\right.$ for $\left.4 \mathrm{~min}\right)$. Following 14 cycles, enzyme was inactivated at $99^{\circ} \mathrm{C}$ for $10 \mathrm{~min}$ and reactions were held at $4^{\circ} \mathrm{C}$ until use.

Preamplification reactions utilized the same primers analyzed to use minimal amounts of material while increasing sensitivity of detection. The reaction was kept at $-20^{\circ} \mathrm{C}$ until ready to use.

PCR. We used the following Human TaqMan ${ }^{\circledR}$ Gene Expression Assays: HPRT1 (Hs02800695_m1), MMP2 (Hs01548727_m1), MMP7(Hs01042796_m1 ), MMP9 (Hs00234579_m1), $\bar{M} M P 14\left(\mathrm{Hs} 01037003 \_\mathrm{g} 1\right)$ all from Life Technologies (Grand Island, NY). A final volume of $10 \mu \mathrm{L}$ was loaded into each well after combination of $5 \mu \mathrm{LTaqMan}{ }^{\circledR}$ Universal Master Mix, $2.5 \mu \mathrm{L}$ cDNA, $0.5 \mu \mathrm{Lprimers}$ and $2.0 \mu \mathrm{L}$ Nuclease-Free water. White, 96-well PCR plates were run using Roche ${ }^{\circledR}$ LightCycler 480 using TaqMan ${ }^{\circledR}$ protocol: reaction was initiated at $50^{\circ} \mathrm{C}$ for $2 \mathrm{~min}$ followed by $95^{\circ} \mathrm{C}$ for $10 \mathrm{~min}$ before $40 \mathrm{PCR}$ cycles were conducted at $95^{\circ} \mathrm{C}$ for $15 \mathrm{~s}$ and $60^{\circ} \mathrm{C}$ for $1 \mathrm{~min}$. Values greater than 35 were excluded due to low confidence. Data was analyzed using the Comparative $\mathrm{C}_{\mathrm{T}}\left(\Delta \Delta \mathrm{C}_{\mathrm{T}}\right)$ Method where $\Delta \mathrm{C}_{\mathrm{T}}$ is the difference between gene-of-interest (GOI) and housekeeping gene (HKG) and $\Delta \Delta \mathrm{C}_{\mathrm{T}}$ is the difference between experimental (i.e. MMP2/9I) and untreated. Fold change is determined by 2 raised to the negative

$\Delta \Delta \mathrm{CT}$ value $\left[2^{(-\Delta \Delta \mathrm{CT})}\right]^{192,193}$. For more information, please see Appendix Table A-1.

\section{siRNA experiments}

Y79 Rb cells were plated overnight in 6-well plates at a cell density of $2.5 \times 10^{5}$ cells per well in $2 \mathrm{~mL}$ RPMI/10\% FBS (no antibiotics) final volume. Two solutions were made: solution A contained $0.75 \mu \mathrm{g}$ of siRNA into $100 \mu \mathrm{L}$ of siRNA Transfection Medium (sc-36868; Santa Cruz Biotechnology) per well; solution B contained $6 \mu \mathrm{L}$ of siRNA Transfection Reagent (sc-29528; Santa Cruz Biotechnology) into $100 \mu \mathrm{L}$ siRNA Transfection Medium per reaction. Silencers: MMP2: sc29398; MMP9: sc-29400; both from Santa Cruz Biotechnology. Solutions A and B were mixed and incubated at RT for 30min. Cells were harvested and washed in siRNA Transfection Medium. We proceeded to resuspend harvested cells in $800 \mu \mathrm{L}$ of siRNA Transfection Medium per well. We added the mixture of solutions A and B onto the cells, mixed gently and incubated for $24 \mathrm{~h}$ at $37^{\circ} \mathrm{C} / 5 \% \mathrm{CO}_{2}$. Next, we added $1 \mathrm{~mL}$ of $\mathrm{RPMI} / 20 \%$ 
FBS without removing the transfection mixture and incubated cells for an additional $24 \mathrm{~h}$ prior to performing functional assays. As a control, we used a scramble sequence (sc37007; Santa Cruz Biotechnology) that does not lead to degradation of any known cellular mRNA.

\section{Protein assessment}

Enzyme-linked immunosorbent assays (ELISA). Human MMP-2 (\#KHC3081; ThermoFisher Scientific), human MMP-9 (\#BMS2016-2; ThermoFisher Scientific), human VEGF (\#BMS277-2; ThermoFisher Scientific), and universal TGF- $\beta 1$ (\#BMS249-4; ThermoFisher Scientific) ELISA kits were purchased from Life Technologies. Human Angiopoietin-2 was purchased from Sigma-Aldrich (St. Louis, MO, RAB0016-1KT). All assays used according to manufacturer's instructions. Biological replicates of cell lysates (25 $\mu \mathrm{g}$ for MMP-2 and MMP-9; $40 \mu \mathrm{g}$ for VEGF and TGF- $\beta 1$ ) were assayed in triplicates. After the addition of the samples, all plates were incubated on a shaker at RT for $2 \mathrm{~h}$, according to instructions. Plates were washed with $1 \mathrm{X}$ wash buffer and incubated with $100 \mu \mathrm{L}$ of their Biotin Conjugate on a shaker for $1 \mathrm{~h}$ at RT followed by addition of $100 \mu \mathrm{L} 1 \mathrm{X}$ Streptavidin-HRP at RT for 30min. In the TGF- $\beta 1$ Kit, these two steps were combined for a $3 \mathrm{~h}$ incubation as indicated by the protocol. Afterwards, $100 \mu \mathrm{L}$ of stabilized chromogen were added to each well and incubated in the dark for $30 \mathrm{~min}$ at RT followed by addition of $100 \mu \mathrm{L}$ stop solution $(0.16 \mathrm{M}$ sulfuric acid) prior to measuring O.D. at $405 \mathrm{~nm}$.

Western blot assays. Cells were initially washed and pelleted prior to addition of ice cold RIPA Buffer (\#89900; Life Technologies). Mixtures were placed on ice for $15 \mathrm{~min}$ before being vortexed for $10 \mathrm{~s}$ and placed in a centrifuge at full speed for $5 \mathrm{~min}$. Supernatant (lysate) was kept and cell debris was discarded ${ }^{194}$. Protein concentrations were calculated using the Pierce ${ }^{\mathrm{TM}}$ BCA Protein Assay Kit (Thermo Scientific). A total of $50 \mu \mathrm{g}$ of denatured protein (denatured using Bolt ${ }^{\circledR}$ Reducing Agent) was used for each sample loaded in a Bolt ${ }^{\mathrm{TM}}$ 4-12\% Bis-Tris Plus Gel (\#NW04125BOX; Invitrogen). Membrane was blocked in $20 \mathrm{~mL}$ of Pierce ${ }^{\mathrm{TM}}$ Fast Blocking Buffer (\#37575; ThermoFisher Scientific) followed by incubation with antibodies. Primary antibodies used: MMP-2 (clone D8N9Y) rabbit monoclonal antibody at 1:1000, MMP-9 rabbit polyclonal antibody at 1:1000, E2F rabbit polyclonal antibody at 1:1000, and $\beta$-Actin (clone D6A8) rabbit monoclonal antibody HRP conjugated at 1:1000. Secondary antibody was Anti-rabbit IgG, HRP-linked at 1:2000. All antibodies were from Cell Signaling Technologies ${ }^{\circledR}$ (Danvers, Massachusetts, USA). We used the Biotinylated Protein Ladder Detection Pack (Cell Signaling Technologies ${ }^{\circledR}$ ), which includes the biotinylated protein ladder and the anti-biotin, HRP-linked antibody. SuperSignal West Pico Chemiluminiscent Substrate (Thermo Scientific) was used to develop the signal. Densitometry analysis was done using Kodak Molecular Imager comparing mean band density ${ }^{195-197}$. For more information, please see Table A-2.

Bicinchoninic acid (BCA) protein concentration assay. Albumin standards were generated ranging from $2000 \mu \mathrm{g} / \mathrm{mL}$ to $25 \mu \mathrm{g} / \mathrm{mL}, 0 \mu \mathrm{g} / \mathrm{mL}$ and $20 \mu \mathrm{L}$ of prepared 
standards were plated on a 96-well microplate. A volume of $2 \mu \mathrm{L}$ of fresh lysate was added (in triplicate) followed by addition of $200 \mu \mathrm{L}$ BCA working solution (50:1, Reagent $\mathrm{A}: \mathrm{B})$. Plates was mixed gently on the benchtop prior to incubation at $37^{\circ} \mathrm{C}$ for $30 \mathrm{~min}$. Plate was then read on a spectrophotometric microplate reader (BioTek $\mu$ Quant, BioTek Instruments, USA) at $562 \mathrm{~nm}$. Using linear fit, protein concentrations were generated in $\mu \mathrm{g} / \mu \mathrm{L}$.

\section{Cellular proliferation}

Quantitation of cell proliferation and viability was performed through use of CellTiter $96^{\circledR}$ AQueous Non-Radioactive assay (MTS) (G5421; Promega, Madison, Wisconsin, USA) following manufacturer's suggested guidelines. Briefly, $5.0 \times 10^{4}$ Y79 and Weri-1 Rb cell lines were cultured per well under different culture conditions: untreated and both MMP2I and MMP9I from concentrations ranging from 500nM to $25 \mu \mathrm{M}$. CellTiter $96^{\circledR} \mathrm{AQ}$ ueous was added at a concentration of $10 \mu \mathrm{L}$ of reagent per $100 \mu \mathrm{L}$ volume per well at specific time points of 0-, 48-, 72-, 96- and $120 \mathrm{~h}$ after culture. After addition of CellTiter reagent, cells were incubated at $37^{\circ} \mathrm{C} / 5 \% \mathrm{CO}_{2}$ for an additional $2 \mathrm{~h}$ before absorbance was read at $485 \mathrm{~nm}$ using $630 \mathrm{~nm}$ as a reference on a spectrophotometric microplate reader (BioTek $\mu$ Quant, BioTek Instruments, USA).

\section{Cell cycle analyses}

Y79 cells were plated under different cell culture conditions overnight at $37^{\circ} \mathrm{C} / 5 \% \mathrm{CO}_{2}$. Next day cells were then harvested and fixed in $200 \mu \mathrm{L} \mathrm{PBS} / 2 \%$ paraformaldehyde (PFA) for $15 \mathrm{~min}$ on ice, then washed and permeabilized using $0.1 \%$ Triton $^{\mathrm{TM}}$ X-100 (Sigma-Aldrich) for 20min. We used far-red fluorescent DNA dye, DRAQ5 $^{\text {TM }}$ (BioLegend, San Diego, CA, USA), at a 1:100 concentration in PBS/1\% FBS for $15 \mathrm{~min}$ on ice to assess cell cycle progression. This is a cell-permeant DNA binding anthraquinone dye, which intercalates between adenine and thymine (A-T) bases of double stranded DNA. DRAQ5 ${ }^{\mathrm{TM}}$ was excited at $642 \mathrm{~nm}$ and acquired using a 642 to $740 \mathrm{~nm}$ filter on the Amnis FlowSight ${ }^{\circledR}$ imaging cytometer (Amnis Corporation, EMD Millipore, Seattle, WA, USA). Sample data acquisition was done using INSPIRE software (Amnis Corporation). Initial acquisition gates were done using dot plots of Brightfield Aspect Ratio vs. Area to separate and identify single cell populations from doublets and debris. Next, a DRAQ5 Aspect Ratio vs. Intensity dot plot was generated to positively identify healthy Y79 cells. Once cell population was identified and acquired, a Bright Field Gradient root mean square (RMS) vs. Normalized Frequency histogram was generated to identify cell population in focus. From there, a histogram of DRAQ5 Intensity vs. Normalized Frequency was created to examine phases of cell cycle $\left(\mathrm{G}_{0} / \mathrm{G}_{1}\right.$ vs. $S$ vs. $\left.\mathrm{G}_{2} / \mathrm{M}\right)$. Percentages and statistical report were generated in IDEAS v6.2 software (Amnis Corporation). 


\section{Migration and invasion assays}

Migration analysis via the wound healing assay. CytoSelect ${ }^{\mathrm{TM}} 24-\mathrm{we}$ ll Would Healing Assay kit was purchased from Cell Biolabs Inc. (CBA-120; San Diego, CA). The 24-well plate was pretreated with $500 \mu \mathrm{L}$ of $0.1 \mathrm{mg} / \mathrm{mL}$ Poly-L-Lysine hydrobromide (Sigma-Aldrich) per manufacturer's instructions and incubated at $37^{\circ} \mathrm{C}$ for $1 \mathrm{~h}$. Wells were washed with distilled, sterile water twice and dried in the biosafety cabinet for $2 \mathrm{~h}$. We added $500 \mu \mathrm{L}$ of $1 \mathrm{X}$ attachment factors (S006100, Life Technologies) containing gelatin (substrate of both MMP-2 and MMP-9) per well and incubated at $37^{\circ} \mathrm{C}$ for $30 \mathrm{~min}$. Solution was aspirated and replaced by $\mathrm{Rb}$ cells at a concentration of $1.0 \times 10^{6}$ cells $/ \mathrm{mL}$. Cell culture conditions included untreated, MMP2I, and MMP9I, both inhibitors at a final concentration of $5 \mu \mathrm{M}$. We ensured cells were evenly distributed and incubated the plate at $37^{\circ} \mathrm{C}$ to create a $95 \%$ confluent monolayer of cells. The inserts were removed; wells were washed twice with distilled, sterile water to remove unattached cells and debris. The cells were then resuspended in $500 \mu \mathrm{L}$ of respective culture conditions. Pictures were taken and 0-, 2-, 6-, 24-, and 48h time points and analyzed for cell migration using an Axiovert 40 CFL (Zeiss, Germany) at a $12.5 \mathrm{X}$ total magnification (lens $2.5 \mathrm{X}$, objective $10 \mathrm{X}$, and camera $0.5 \mathrm{X})$.

Invasion assay. CytoSelect ${ }^{\mathrm{TM}}$ Cell Invasion Assay kit was purchased from Cell Biolabs Inc (CBA-110, San Diego, CA). We use the provided $8 \mu \mathrm{m}$ pore polycarbonate membrane coated with basement membrane matrix solution. Rb cell suspension (in serum free media) was placed in the upper chamber to determine the invasion capacity of the cells after degradation of the matrix membrane proteins $6 \mathrm{~h}$ post culture. Invasive cells were stained using $400 \mu \mathrm{L}$ of the included Cell Stain Solution and incubated at RT for $10 \mathrm{~min}$ before being washed with distilled water. Invasive cells were then quantified with a light microscope under $100 \mathrm{X}$ total magnification (lens $2.5 \mathrm{X}$, objective $40 \mathrm{X}$ ), with 4 individual fields per insert. Inserts were placed to wells containing $200 \mu \mathrm{L}$ of Extraction Solution followed by $10 \mathrm{~min}$ incubation at RT on an orbital shaker. Quantitation of cells measured at OD 560nm using spectrophotometer (BioTek $\mu$ Quant, BioTek Instruments, USA).

\section{Statistical analysis}

Data on bar graphs are expressed as means $\pm \mathrm{SD}$ or $\pm \mathrm{SEM}$ (as indicated), with $p<0.05$ considered statistically significant. The data were compared where appropriate by paired Student $t$ test or by the Holm-Sidak Method, with alpha $=5.0 \%$, using Graph Pad Prism v5. 


\section{Results}

\section{Inhibition of MMP-2 and MMP-9 decreases migration in the metastatic Y79 Rb cell line, and viability in the non-metastatic Weri-1 model}

Tumor migration and invasion of the optic nerve and the uvea has a significant impact in the prognosis of $\mathrm{Rb}$ as it denotes metastatic disease often including distant sites such as the brain, lungs and/or bones that may be fatal to diseased patients. Ultimately, metastatic disease is much more difficult to treat due to lack of effective local therapies and often includes much more aggressive and life-threatening treatment options. Previous work demonstrated high expression of both MMP-2 and MMP-9 in extra-ocular Rb as well as those with optic nerve involvement and worse staging ${ }^{191}$. To investigate the effects of inhibition of MMP-2 and MMP-9 on the migration of Rb cells we used an established in vitro system consisting of well characterized Rb cell lines. This system represents both a metastatic model, represented by the Y79 cell line, and a non-metastatic model, represented by the Weri-1 cell line. The pathogenicity and aggressiveness of these cells have been established in an in vivo animal model ${ }^{37}$. This in vivo study consisted of intravitreal injection of $2 \times 10^{4}$ Y 79 or Weri-1 cells into mice and found Y79 readily invaded the retina, subretinal space, anterior chamber, optic nerve head, choroid, contralateral eye, subarachnoid space, and the brain. Meanwhile, Weri-1-injected mice displayed little to known invasion and only showed choroidal invasion during late stage disease $^{37}$. The cell lines followed the migration and aggressive patterns from which they were derived, Y79 from a patient that ultimately developed brain metastasis and Weri-1 from a patient without metastatic disease. To test the hypothesis that MMPs would control $\mathrm{Rb}$ cell migration and invasion, $\mathrm{Rb}$ cell lines were added to the upper chamber of an $8 \mu \mathrm{m}$ polycarbonate membrane coated with basement membrane proteins in serum free RPMI media at a density of $1 \times 10^{6}$. The lower chamber had media in the presence or absence of matrix metalloproteinases inhibitors (MMPI). We used ARP100 as an inhibitor of MMP-2 at a $5 \mu \mathrm{M}$ concentration; and AG-L-66085 as a MMP-9 inhibitor at a $5 \mu \mathrm{M}$ concentration, as described in other studies ${ }^{198}$. If MMP signaling is required for migration we would observe a reduction in $\mathrm{Rb}$ cell migration through the basement membrane after treatment with MMPI. Our results showed a significant reduction of $\mathrm{Rb}$ cell migration through the basement membrane, or extracellular matrix (ECM), after MMPI treatment. These results suggest MMP-2 and MMP-9 activity are necessary to degrade ECM and promote cellular invasion in Rb. In Figure 2-1 we show a representative field for each insert. Next, we performed quantitation analyses via colorimetric change as indicated with Cell Staining Solution and measured on a spectrophotometric microplate reader. As shown in Figure 2-1 there is a significant difference between untreated Y79 and those treated with the MMPI (Y79 Rb cells, Untreated versus MMP2I: $0.397 \pm 0.06$ versus $0.260 \pm 0.010, p=0.01$; versus MMP9I: $0.225 \pm 0.005, p=0.0009$; Weri-1 Rb cells, Untreated versus MMP2I: $0.164 \pm 0.028$ versus $0.061 \pm 0.014, p=0.043$; versus MMP9I: $0.056 \pm 0.018, p=0.0294$ ). To confirm our results, we used a different method, the classic wound healing assay. We adhered $\mathrm{Rb}$ cells to poly-L-lysine hydrobromide coated surfaces and created artificial wounds of 

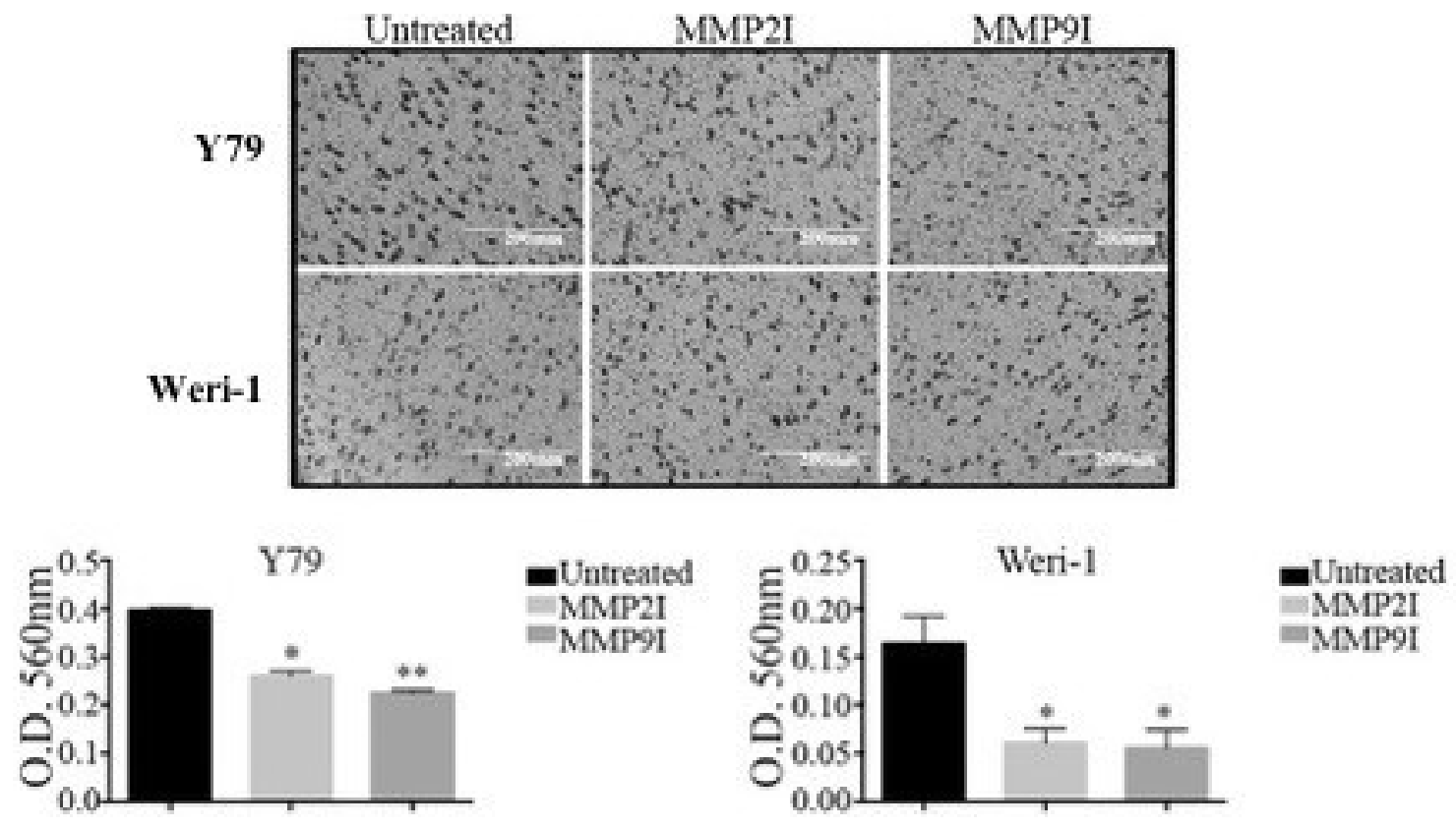

Figure 2-1. Inhibition of MMP-2 and MMP-9 reduced Rb invasion.

Y79 and Weri-1 cells were added to the upper chamber of an $8 \mu \mathrm{m}$ polycarbonate membrane coated with basement membrane in serum-free media. The lower chamber contained cell culture media with or without MMPI. Six-hours post culture, invasive cells degraded the ECM and were collected, stained and counted. Representative figures are shown with a 100X total magnification. Cells were extracted, and OD measured for Y79 and for Weri-1. For each condition $n=3$; gap was measured in 5 different points. Modified with permission from BMC Cancer. Webb AH, Gao BT, Goldsmith ZK, et al. Inhibition of MMP-2 and MMP-9 decreases cellular migration, and angiogenesis in in vitro models of retinoblastoma. BMC Cancer. 2017;17(1):434. Copyright (June 20, 2017), with permission through Creative Common CC-BY. 
approximately $900 \mu \mathrm{m}$. The closure of the gap area was measured at different time intervals for up to $48 \mathrm{~h}$, as this represents the potential of cells to readily migrate. We observed Y79 untreated cells closed the gap area (Figure 2-2) while MMP2I and MMP9I-treated Y79 cells showed a significant reduction in migration (Untreated versus MMP2I at 24h: $315 \pm 45$ versus $742.5 \pm 22.5, p=0.0001$; versus MMP9I: $810 \pm 36.7, p=0.0001)$. Migration potential as measured by the wound-healing assay revealed that inhibition of either MMP-2 or MMP-9 caused a significant reduction of Y79 cells migration.

Previous reports have demonstrated MMP inhibitors may have cytostatic and cytotoxic effects on cancer cells. The exact mechanism(s) behind these observations is not well understood. Nonetheless, we hypothesized inhibition of MMP-2 or MMP-9 by the pharmacological inhibitors may, in turn, decrease cellular viability. To investigate this hypothesis, we treated cells under the same conditions as mentioned above and utilized the CellTiter $96^{\circledR} A Q_{\text {ueous }}$ Non-Radioactive assay (MTS). The cellular viability assays showed both MMP2I and MMP9I significantly reduced the viability of Y79 cells (Figure 2-3) (Untreated versus MMP2I: $116.67 \% \pm 1.40$ versus $42.66 \% \pm 1.4, p<0.005$; versus MMP9I: $32 \% \pm 0, p<0.005)$. We next hypothesized that this reduction in viability would be the result of exit from the cell cycle (cytostatic) or cell death (apoptosis). We examined cell cycle using a nuclear dye and imaging flow cytometry to determine in which phase of the cell cycle these Rb cells were in and expected to see higher percentages of cells in $\mathrm{G} 0 / \mathrm{G} 1$ phase. In addition to the cytotoxic effect we observed a significant increase in the percentage of cells within the G0/G1 cell cycle phase in Y79 cells treated with MMP9I compared to those untreated (Figure 2-3)[Untreated versus MMP9I: G0/G1 phase: $32.44 \% \pm 0.907$ versus $49.51 \pm 1.059$; $\mathrm{S}$ phase: $5.23 \% \pm 0.165$ versus $5.28 \% \pm 0.062 ; \mathrm{G} 2 / \mathrm{M}$ phase: $5.16 \% \pm 0.117$ versus $4.252 \% \pm 0.335]$.

Prior studies have demonstrated a lack of invasion and migration of Weri-1 cells in vivo, especially when compared to Y79, and found similar patterns of migration / invasion to the tumors from which these cells were derived. Mice inoculated with Weri-1 cells readily form primary tumors, but these tumor cells rarely invade and only migrate small distances anteriorly. Furthermore, previous in vitro work has also demonstrated a Weri-1-specific lack of binding to choroidal and glioma cells, further demonstrating a lack of binding for Wer-1. It is possible Weri-1 lack the necessary cytoskeletal molecular machinery to initiate and undergo metastasis. It is for this reason, unfortunately, we were not able to carry out the migration assay using Weri-1 cells because these cells detached from the surface of the wells after treatment with either of the inhibitors (Figure 2-2), which precluded any meaningful measurement. To better understand why they detached we did a titration assay using a $500 \mathrm{nM}$ to $25 \mu \mathrm{M}$ range of the MMPIs to investigate the sensitivity of Weri-1 Rb cells to MMP2I (left) and MMP9I (right) (Figure 2-4) and found Weri-1 Rb cells are sensitive to inhibitors even at low concentrations.

Collectively, these findings support the conclusion that MMP-2 and MMP-9 activity stimulates $\mathrm{Rb}$ cell migration in vitro and that similar pathways could be involved in $\mathrm{Rb}$ metastasis in vivo. 


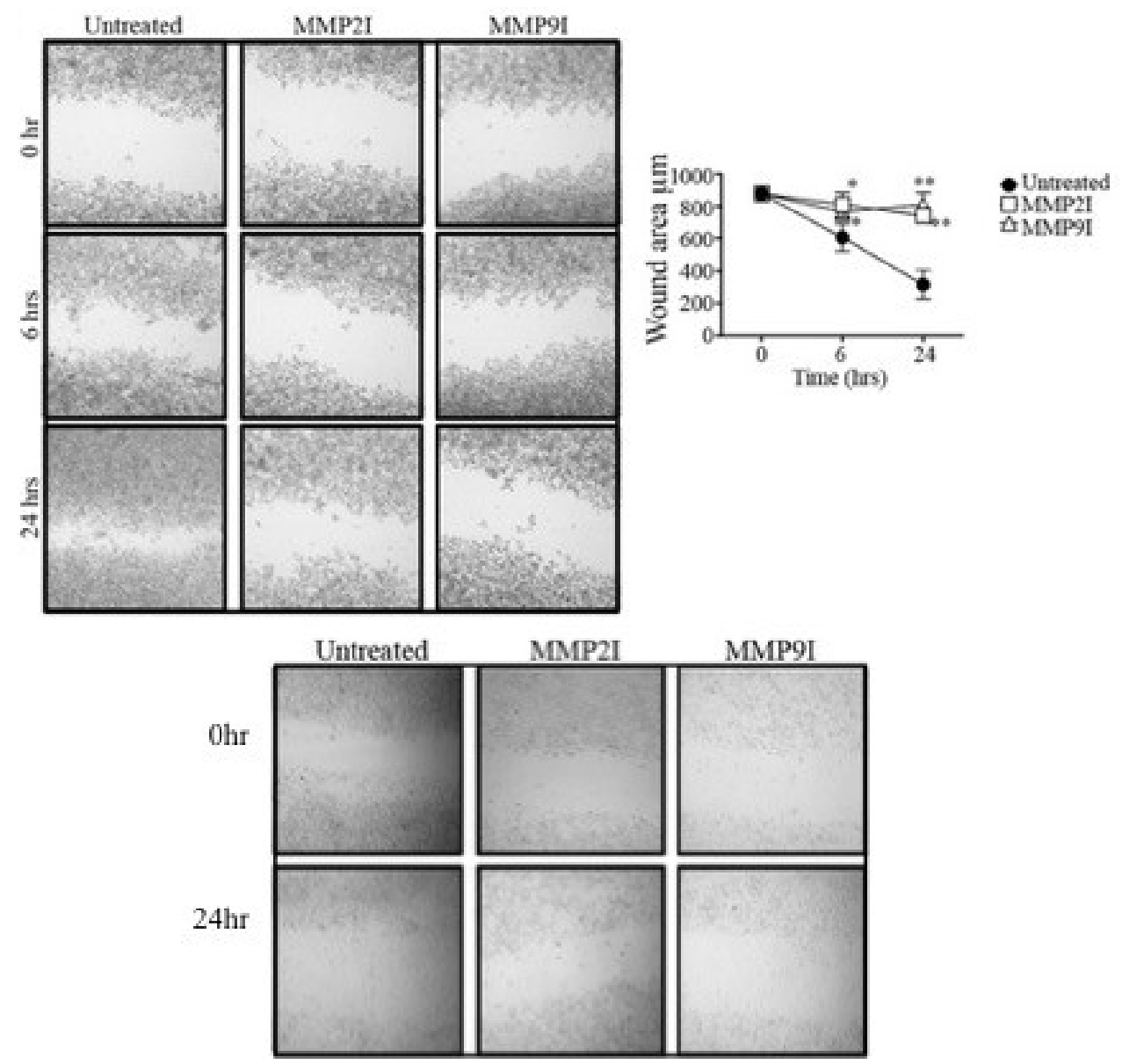

Figure 2-2. Inhibition of MMP-2 and MMP-9 reduced Rb cell migration.

Y79 Rb cells were cultured in the presence or absence of MMP-2 or MMP-9 inhibitors for $48 \mathrm{~h}$ on poly-L-lysine coated wells with gelatin as substrate. Sterile in-well inserts created a gap of $900 \mu \mathrm{m}$. Gap closure was recorded at different time intervals using an Axiovert 40 CFL (top left). Total magnification is $12.5 \mathrm{X}$. Plotted results are in shown (top right). Weri-1 cells showed increased cell death and detachment from coated surface (bottom). For each condition $n=3$; gap was measured in 5 different points. Modified with permission from BMC Cancer. Webb AH, Gao BT, Goldsmith ZK, et al. Inhibition of MMP-2 and MMP-9 decreases cellular migration, and angiogenesis in in vitro models of retinoblastoma. BMC Cancer. 2017;17(1):434. Copyright (June 20, 2017), with permission through Creative Common CC-BY. 

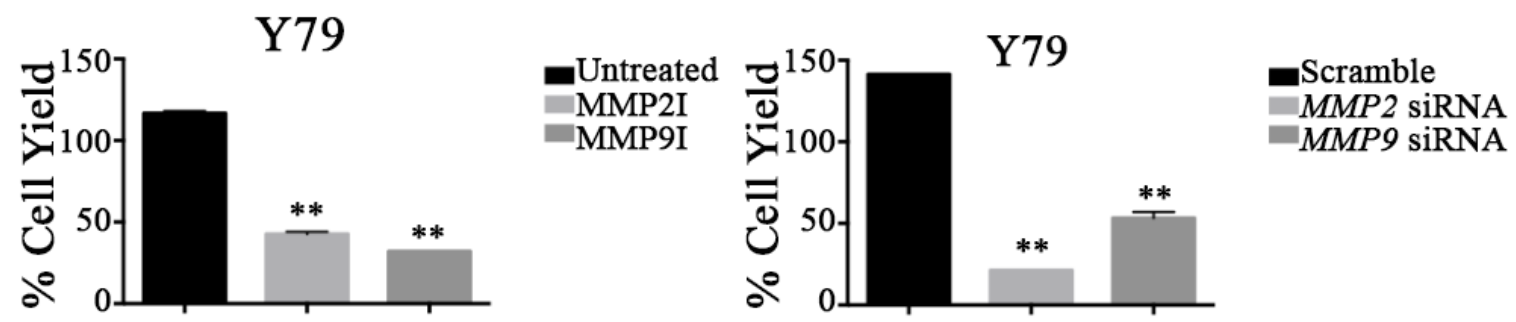

$\mathrm{C}$

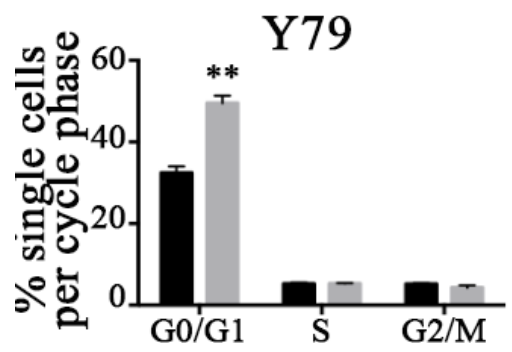

Untreated

MMP9I

Figure 2-3. Inhibition of MMP-2 or MMP-9 reduced Rb cell viability and cell cycle progression.

Y79 cells were cultured in the presence or absence of the MMPI overnight. Next day, we collected cells and assessed viability by Trypan Blue exclusion. (A) Chemical inhibition of Y79 with MMPI significantly reduced cell yield when compared to untreated cells. (B) RNA interference was used to confirm on-target effects of MMPIs. Y79 were cultured in the presence of either $M M P 2$ or $M M P 9$ siRNA. $M M P 2$ and $M M P 9$ knockdown groups showed significant reduction in cell yield, illustrating an on-target effect of MMPI. (C) Imaging flow cytometry analysis showed inhibition of MMP9 prevents progression of $\mathrm{Rb}$ cell division using nuclear DRAQ5 ${ }^{\mathrm{TM}}$ labeling (bottom). Bar graphs indicate results \pm SEM to control. ${ }^{* *} p<0.005$. Modified with permission from BMC Cancer. Webb AH, Gao BT, Goldsmith ZK, et al. Inhibition of MMP-2 and MMP-9 decreases cellular migration, and angiogenesis in in vitro models of retinoblastoma. BMC Cancer. 2017;17(1):434. Copyright (June 20, 2017), with permission through Creative Common CC-BY. 

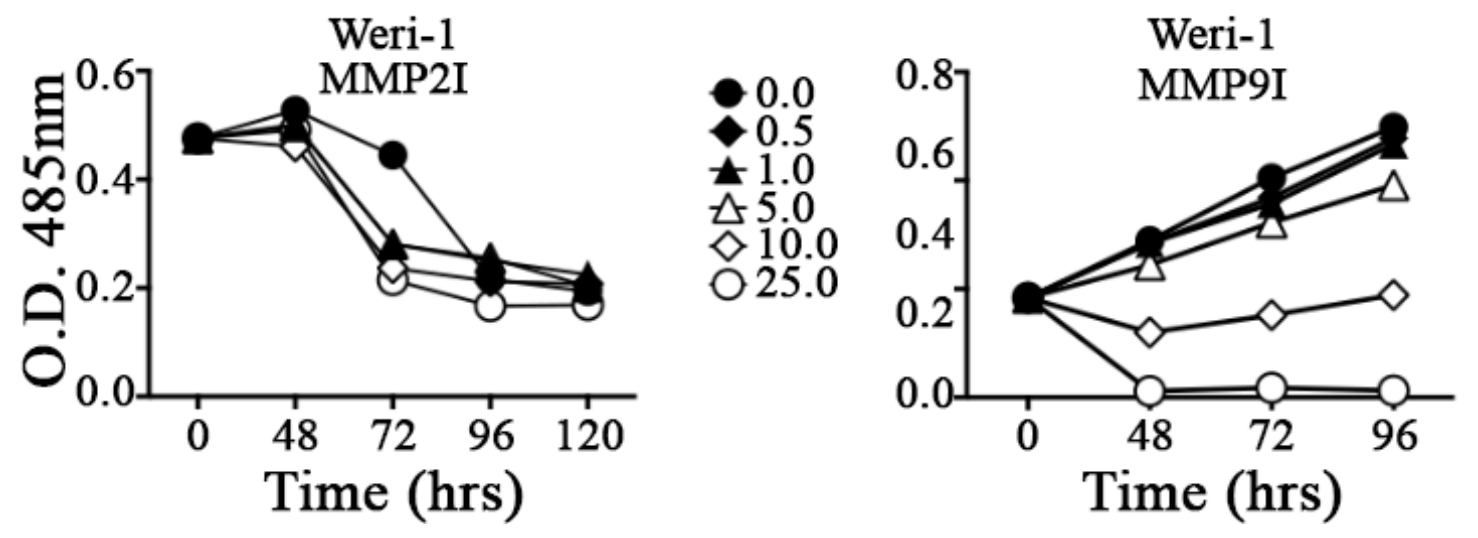

Figure 2-4. Weri-1 Rb cells are sensitive to MMPIs.

Weri-1 cells were cultured in the presence or absence of MMPI. The MMPI were used at a concentration range of $500 \mathrm{nM}$ to $25 \mu \mathrm{M}$ for up to $120 \mathrm{~h}$. MTS proliferation solution was added to each well at a concentration of $10 \mu \mathrm{L}$ solution per $100 \mu \mathrm{L}$ at specific time points $(0-, 48-, 72-, 96-$, and $120 \mathrm{~h})$ and incubated at $37^{\circ} \mathrm{C} / 5 \% \mathrm{CO}_{2}$ for $2 \mathrm{~h}$ prior to reading on an absorbance reader. Values represent are optical density (O.D.) \pm SEM at 482nm with a reference wavelength of $630 \mathrm{~nm}$. Modified with permission from BMC Cancer. Webb AH, Gao BT, Goldsmith ZK, et al. Inhibition of MMP-2 and MMP-9 decreases cellular migration, and angiogenesis in in vitro models of retinoblastoma. BMC Cancer.

2017;17(1):434. Copyright (June 20, 2017), with permission through Creative Common CC-BY. 


\section{Downregulation of MMP-2 and MMP-9 by pharmacological inhibitors in Y79 cells}

Next, we hypothesized that Y79, considered the more aggressive, metastatic model for $\mathrm{Rb}^{37}$, has higher levels of $M M P 2$ and $M M P 9$ at mRNA and protein levels compared to the non-metastatic Weri-1. This hypothesis stemmed from a previous ex vivo study using tissue of patients with metastatic Rb and both MMP-2 and MMP-9 were readily detected in tumors that ultimately metastasized ${ }^{191}$. To address this hypothesis, we investigated the expression of MMPs mRNA by qPCR analysis. Qualitative PCR analysis shown in Figure 2-5A revealed Y79 had higher expression of both $M M P 2$ and $M M P 9$ mRNA transcripts compared to Weri-1, as we hypothesized (Y79, MMP2: $4.116 \pm$ 0.3, MMP9: $7.186 \pm 0.4$; Weri-1, MMP2: $2.1 \pm 0.4, M M P 9: 3.78 \pm 0.4$ ). Additional analyses were performed to investigate if other MMPs associated with tumor invasion ${ }^{199}$ were expressed in these $\mathrm{Rb}$ cell lines. We found no detection (ND) of MMP7 mRNA but found expression of MMP14 (7.96 \pm 0.8$)$ in Y79 cells. Given the recent emphasis in the role of MMP-2 and MMP-9 in ECM degradation and cancer invasion we are focusing our studies on investigating MMP-2 and MMP-9 activity in Rb.

MMP regulation occurs primarily at the transcriptional level ${ }^{200}$. We hypothesized that these selective inhibitors would inhibit $M M P 2$ and $M M P 9$ mRNA transcription that would ultimately lead to decreased MMP-2 and MMP-9 protein levels. To investigate if these inhibitors did block MMP mRNA transcription, we investigated MMP gene expression in both $\mathrm{Rb}$ cell lines in the cells treated with respective MMPIs relative to untreated (baseline) cells. As shown in Figure 2-5B there was a significant reduction in the mRNA expression of both $M M P 2$ and $M M P 9$ by their respective inhibitors in Y79 cells. Similar results were found in Weri-1 cells (Figure 2-5C). These results confirmed that MMPs regulate Rb cell migration. Due to our laboratory's interests in (1) invasion and tumor aggressiveness, and (2) vitreous seeds, we concentrated the rest of our investigations on Y79, the more aggressive and metastatic $\mathrm{Rb}$ tumor model. While we demonstrated decreases in MMP mRNA levels, we further hypothesized this downregulation would result in decreases of MMP-2 and MMP-9 protein levels, as well. To address this we measured both MMP-2 and -9 levels using two conventional techniques: MMP secretions were measured using ELISAs and intracellular MMP levels were detected using Western blotting. Despite inhibition of MMP2 mRNA, we still observed intracellular protein by Western blot (Wb) analysis (Figure 2-5E) but a significant reduction by ELISA (Figure 2-5G) [Untreated versus MMP2I: $237 \pm 9$ versus $179 \pm 10, p<0.005$; versus MMP9I: $260 \pm 17, p=0.266]$. The difference could stem from the specificity of the assays, as the ELISA measures active enzyme and the $\mathrm{Wb}$ measured total protein. However, treatment with MMP9I showed a significant reduction in MMP-9 intracellular protein by both Wb and ELISA (Figure 2-5E and 5G) and Untreated versus MMP2I: $124 \pm 3$ versus $115 \pm 3, p=0.106$; versus MMP9I: $84 \pm 6, p<0.0005)$.

As demonstrated above, regulation of MMPs occurs at the transcriptional level. One of the currently-understood interactions involving MMP transcriptional activation is through E2F, which is directly involved in MMP gene transcription by binding MMP 
Figure 2-5. Pharmacological inhibitors of MMP-2 and MMP-9 downregulate $M M P 2$ and $M M P 9$ mRNA.

A. The following MMPs were examined at the transcriptional level: $M M P 2, M M P 7$, $M M P 9$, and MMP14. Results show mRNA expression relative to HPRT1. Bar graphs indicate results $\pm \mathrm{SD} ; n=3$. Y79 Rb cells express $M M P 2, M M P 9$, and $M M P 14$; Weri-1 expressed MMP2and MMP9. B,C Y79 (B) and Weri-1 (C) cells were treated with MMP2 and MMP-9 inhibitors overnight. Results show that the inhibitors act at the transcriptional level. Bar graphs indicate fold change $\pm \mathrm{SD} ; n=3$. D. Knockdown of $M M P 2$ and $M M P 9$ by RNA interference shows on-target effects. E,F Reduction of MMP-2 and MMP-9 protein in Rb cells treated with MMPI (E) and siRNA (F); $* p<0.05, * * p<0.005$. Wb bar graphs indicate results \pm SEM ratio of target to $\beta$ actin; $n=3$. G,H ELISAs of MMP-2 and MMP-9 protein of whole cell lysates after treatment with MMPI $(\mathbf{G})$ or siRNA $(\mathbf{H}) ;{ }^{*} p<0.05,{ }^{*} p<0.005$. I,J, E2F regulates MMP expression in Y79 cells. Y79 cells treated with MMPI (I) or with siRNA (J) were assessed by $\mathrm{Wb}$ analysis for E2F. Western blot bar graphs indicate results \pm SEM ratio of target protein to $\beta$-actin; $n=3 ; * * p<0.005$. Modified with permission from BMC Cancer. Webb AH, Gao BT, Goldsmith ZK, et al. Inhibition of MMP-2 and MMP-9 decreases cellular migration, and angiogenesis in in vitro models of retinoblastoma. $B M C$ Cancer. 2017;17(1):434. Copyright (June 20, 2017), with permission through Creative Common CC-BY. 
A

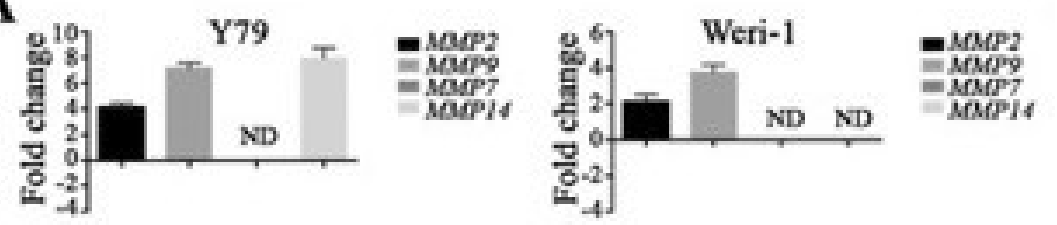

B

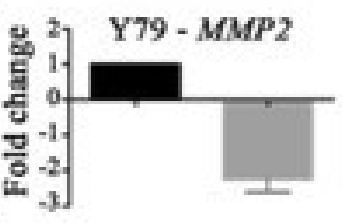

- Vatreaned

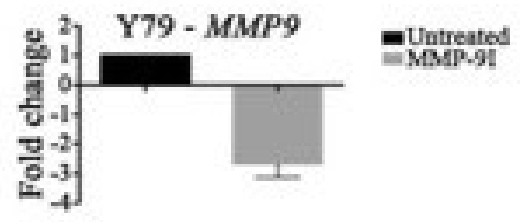

C

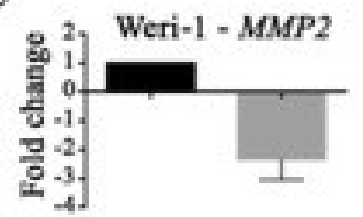

- Yumeaned

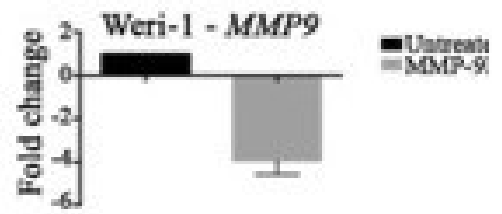

D
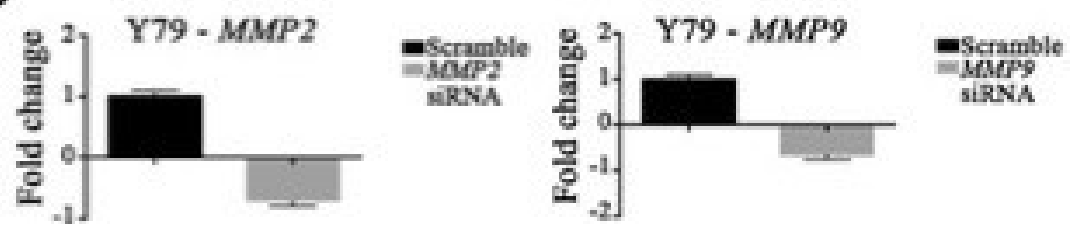

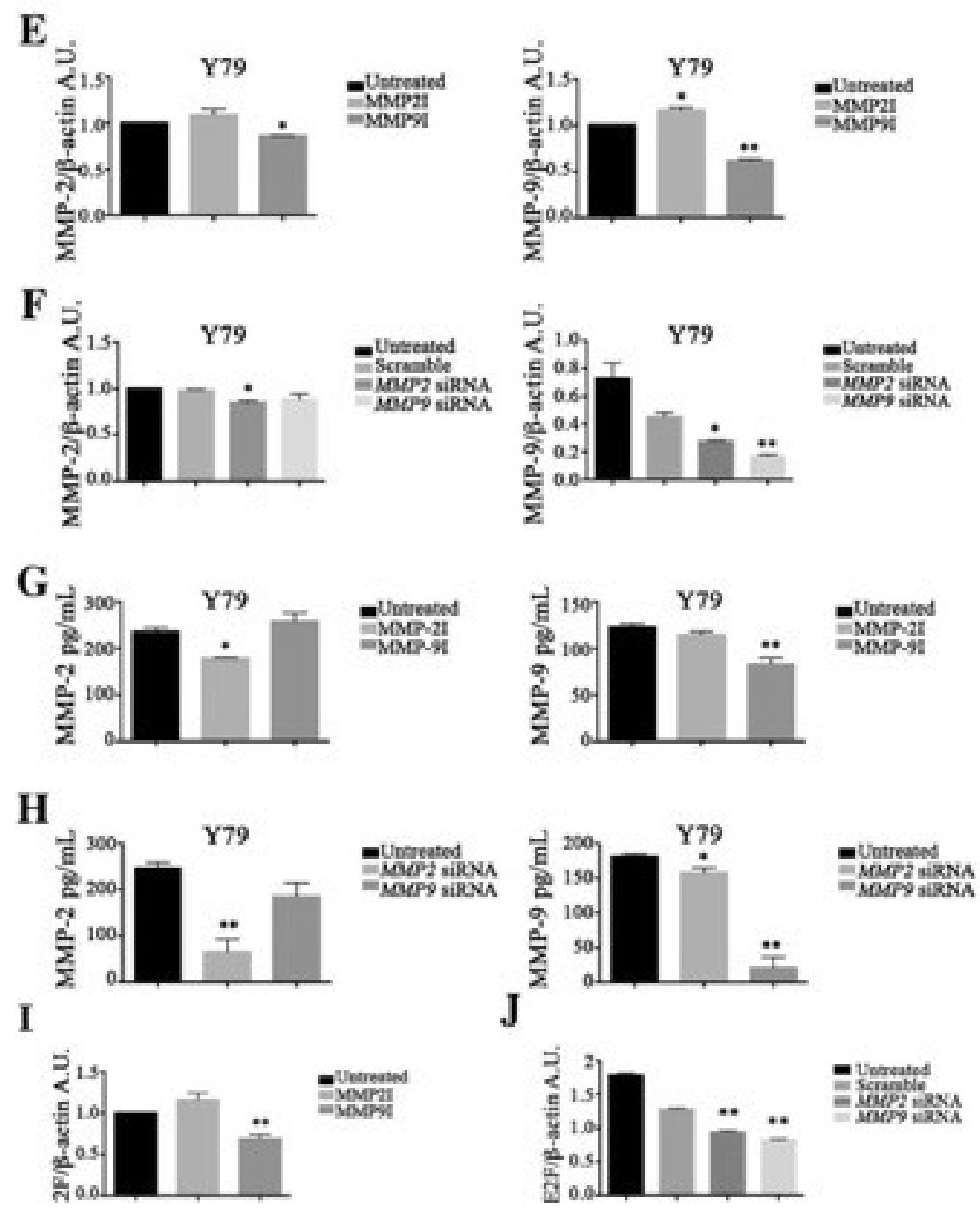

Figure 2-5. (continued). 
promoters and may be activated by MMPs. Classically, E2F belongs to a family of transcription factors that regulate cell cycle and DNA replication in mammalian cells ${ }^{201}$. Therefore, we investigated the expression of E2F in Y79 Rb cells and if treatment with MMPI could modulate their levels. We hypothesized inhibition of MMP2/9 would result in decreased E2F protein. As shown in Figure 2-5I, there is a significant reduction of E2F levels in Y79 cells treated with MMP9I, but not MMP2I, suggesting E2F regulates MMP-9 expression. Next, we investigated if this was an on-target effect of the MMP9I by using siRNA. We targeted $M M P 2$ and $M M P 9$ and confirmed downregulation of their gene expression and proteins levels (Figure 2-5D-H) . The results in Figure

2-5J showed a significant reduction in E2F levels by both $M M P 2$ and $M M P 9$ siRNA compared to the non-specific RNA target or scramble group, suggesting this may not be an off-target effect of downregulation of the MMP-2 and MMP-9 and that E2F disruption is a consequence of MMP inhibition.

\section{Pharmacological inhibition of MMPs reduces secretion of angiopoietin-2, but not VEGF, in Y79 cells}

Retinoblastoma tumors are highly angiogenic. Aqueous humor from enucleated $\mathrm{Rb}$ eyes has been shown to trigger significant angiogenic activity ${ }^{202}$. This angiogenic activity has also been targeted preclinically. Vascular endothelial growth factor (VEGF), known to underlie angiogenic activity, stimulated MMP-9 production in human retinal pigment epithelial cells ${ }^{203}$. Furthermore, other studies have demonstrated some success in decreasing VEGF levels to treat primary tumors in $v i v o^{166}$. To further examine possible mechanisms by which MMPs might stimulate migration and other pro-metastatic processes in Rb disease, we analyzed the effects of MMP inhibition on production of angiogenic factors, including the pro-angiogenic molecules VEGF and Angiopoietin-2. These two molecules are known to function synergistically to promote vessel growth and maturation. Furthermore, the combination of these two proteins has demonstrated to increase MMP-9 levels ${ }^{204}$. As shown in Figure 2-6A, there was no significant reduction in VEGF secretion in Y79 cells after treatment with MMP2I, but there was a significant increase after MMP9I treatment (Untreated versus MMP2I: $366 \pm 44 \mathrm{pg} / \mathrm{mL}$ versus $418 \pm 37 \mathrm{pg} / \mathrm{mL} ; p=0.83$; versus MMP9I: $440 \pm 10 \mathrm{pg} / \mathrm{mL} ; p=0.01$ ). Both VEGF and Angiopoietin-2, or perhaps the equilibrium between the two, influence tumor growth and vascular regression, prompting us to measure the effects of MMPI on Angiopoietin- $2^{205}$. The protein levels of Angiopoietin-2 in Y79 were reduced, although marginally significant, by MMP9I (Figure 2-6B) [Y79 Untreated versus MMP2I: $1120.3 \pm 65 \mathrm{pg} / \mathrm{mL}$ versus $1067.6 \pm 153 \mathrm{pg} / \mathrm{mL}, p=0.552$; versus MMP9I: $990 \pm 90 \mathrm{pg} / \mathrm{mL}, p=0.05]$. In contrast, as shown in Figure 2-6A, the non-metastatic Rb cell line Weri-1 showed a significant reduction in VEGF after MMP9I treatment (Untreated versus MMP2I: $371 \pm 75 \mathrm{pg} / \mathrm{mL}$ versus $270 \pm 95 \mathrm{pg} / \mathrm{mL}, p=0.221$; versus MMP9I: $228 \pm 60 \mathrm{pg} / \mathrm{mL} ; p=0.005$ ) but a significant increase in Angiopoietin-2 (Untreated versus MMP2I: $883 \pm 10$ versus $1190 \pm 13, p<0.005$; versus MMP9I: $1495 \pm 147, p<0.005$ ) after treatment (Figure 2-6B). Collectively, these results showed that in the metastatic Y79 cell line, we observed a significant increase in VEGF by MMP9I, and a reduction, 

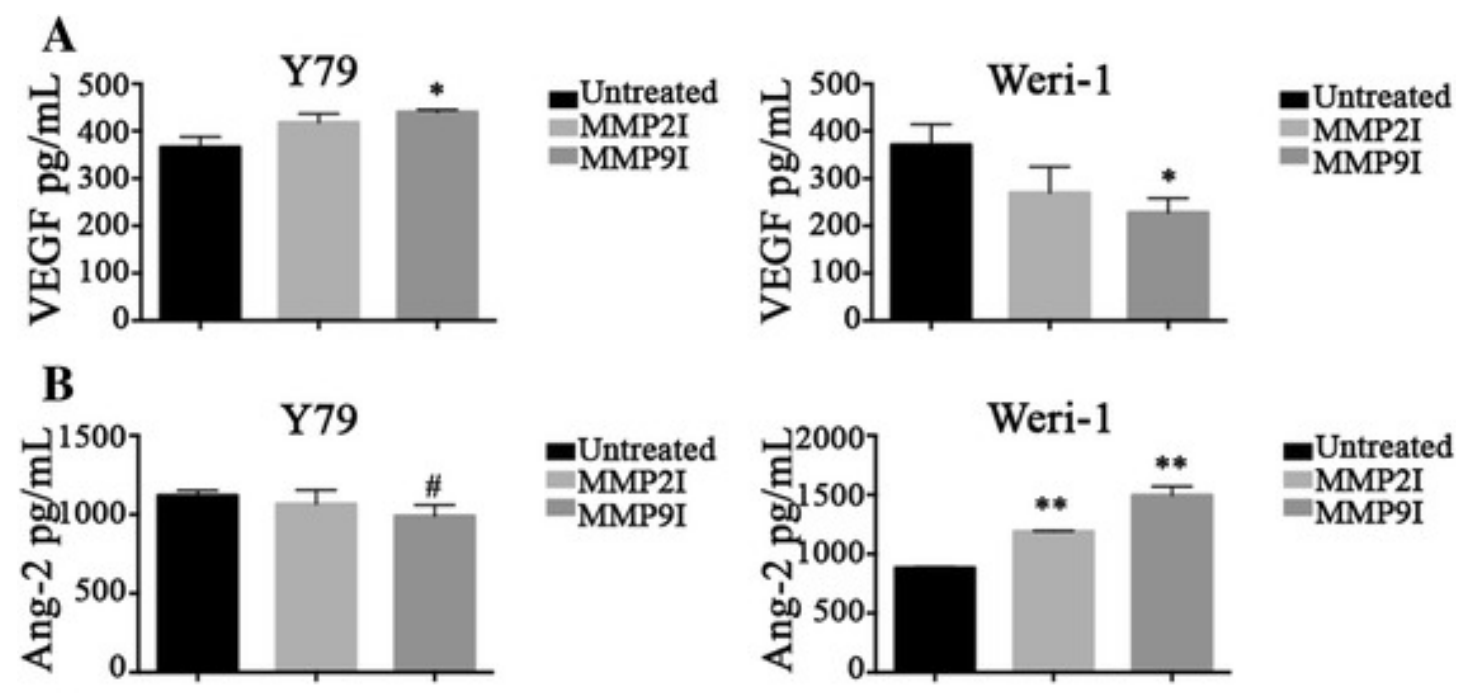

Untreated

MMP2I

MMP9I
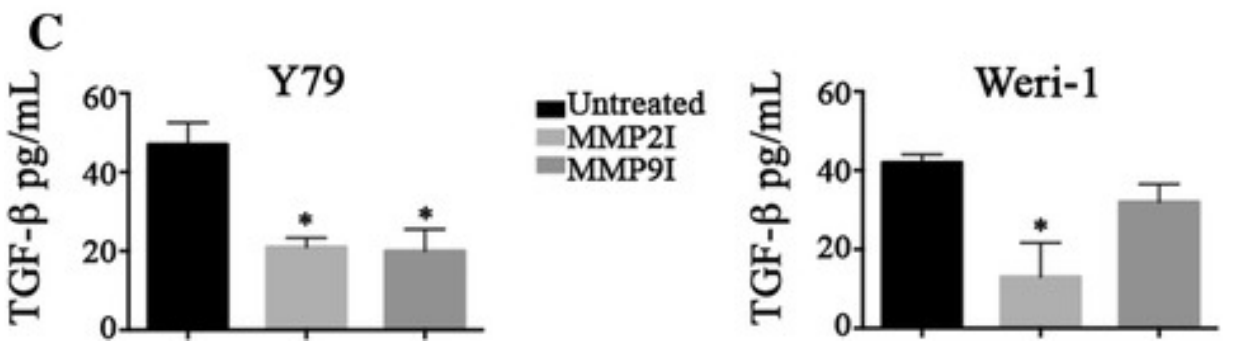

Untreated
MMP2I

MMP9I

Figure 2-6. MMP inhibition reduces angiogenic protein levels.

Y79 and Weri-1 cells were cultured in the presence or absence of the MMPI overnight. Next day, we collected cell lysates (A-B) and supernatants to investigate protein levels by ELISA. A shows VEGF protein levels; B shows Ang-2 protein levels and $\mathbf{C}$, shows levels of TGF- $\beta 1$, an immunomodulator. In all secretion analyses bar graphs indicate results $\pm \mathrm{SD} ; n=3 ;{ }^{*} p<0.05, * * p<0.005, \# p=0.05$. Modified with permission from BMC Cancer. Webb AH, Gao BT, Goldsmith ZK, et al. Inhibition of MMP-2 and MMP-9 decreases cellular migration, and angiogenesis in in vitro models of retinoblastoma. $B M C$ Cancer. 2017;17(1):434. Copyright (June 20, 2017), with permission through Creative Common CC-BY. 
albeit minimal in Angiopoietin-2 ( $p=0.05)$. The opposite was observed in Weri-1, as there was a significant reduction in VEGF by MMP9I and a significant increase in Angiopoietin-2 by MMP2I and MMP9I. These results highlight the complexity associated with $\mathrm{Rb}$ disease and demonstrate how identical molecules play differing roles in the physiology and sustainment of Rb.

While Angiopoietin-2 and VEGF act synergistically to promote angiogenesis, so too, do Transforming Growth Factor-beta 1 (TGF- $\beta 1$ ) and VEGF. Specifically, these molecules are known to promote long-term protection of endothelial cells while promoting angiogenesis ${ }^{206}$. TGF- $\beta 1$ is a potent immunosuppressor of cytotoxic cells by depressing cytolytic ability and thus promoting metastases. Recent work suggests MMPs may stimulate TGF- $\beta 1$ activity ${ }^{201,207}$. To determine if inhibition of MMP-2 or MMP-9 could affect the TGF- $\beta 1$ pathway in Rb, we measured secretion of TGF- $\beta 1$ by Y79 cells after treatment with the inhibitors. As shown in Figure 2-6C TGF- $\beta 1$ secretion was significantly reduced in Y79 cells by either of the inhibitors (Untreated versus MMP2I: $47.0 \pm 11 \mathrm{pg} / \mathrm{mL}$ versus $20.0 \pm 4 \mathrm{pg} / \mathrm{mL}, p=0.010$; versus MMP9I: $20.7 \pm 11 \mathrm{pg} / \mathrm{mL}, p=0.013)$. Similarly, we tested TGF- $\beta 1$ secretion in Weri-1 cells (Figure 2-6C) and found it was significantly reduced after MMP-2 inhibition (Untreated versus MMP2I: $42.0 \pm 4 \mathrm{pg} / \mathrm{mL}$ versus $13.2 \pm 15 \mathrm{pg} / \mathrm{mL}, p=0.012$ ), but not MMP-9 inhibition (Untreated versus MMP9I: $32 \pm 9 \mathrm{pg} / \mathrm{mL}, p=0.088$ ). Here, we demonstrated the convolution associated with metastatic and non-metastatic $\mathrm{Rb}$ cell lines. We found MMP-2 and MMP-9 exert direct activity on the angiogenesis through Angiopoietin-2 and VEGF and immunomodulation through production of TGF- $\beta 1$ as well as migration using $\mathrm{Rb}$ cell lines.

\section{Discussion}

Our work focuses on MMP-2 and MMP-9 activity in Rb, the most common intraocular malignancy in children. Consistent with previous reports, we show MMP-2 and MMP-9 are present in $\mathrm{Rb}$. For the first time in retinoblastoma, we provide in vitro analysis of two cell lines, Y79 and Weri-1, which represent the metastatic and nonmetastatic model for $\mathrm{Rb}$. Prior studies have failed to examine potential functions of the MMPs in Rb. As part of our in-depth analysis we compared both cell lines in their response to several properties: invasion, cellular migration, mRNA expression and protein levels of MMP-2 and MMP-9, the production of the angiogenic factors VEGF and Angiopoietin-2, and the immunomodulatory protein TGF- $\beta 1$.

The outcomes of our experiments revealed differences in several intrinsic properties associated with tumor progression in Y79 and Weri-1. Tumor cells in patients are likely to have diverse cell populations that have varying metastatic potential, thus studying both cell lines provides important insight into actual properties of tumor in vivo. While these two cell types both respond to MMPI, they do so in different ways using different pathways. The MMPI used in this study mediate their effect on Rb cells through inhibition of $M M P 2$ and $M M P 9$ mRNA in both Y79 and Weri-1. However, the effects on 
angiogenic factors differ between cell types and illustrate the complex nature of these molecules.

Our results suggest the mechanisms underlying the production of angiogenic factors are different among these cells. The production of VEGF in Weri-1 may be more dependent on MMP-2 or MMP-9 activity as there was a significant reduction in protein production after treatment with MMP2I and MMP9I. Conversely, production of Angiopoietin-2 is increased in Weri-1 after MMPI treatment suggesting Angiopoietin-2 production is independent of MMP-2 or MMP-9 activity. These results suggest these two angiogenic pathways are not involved in primary actions on metastasis, as Weri-1 is the non-metastatic model. In contrast, Y79 cells showed a significant increase in VEGF production after MMPI treatment, although MMP9I reduced Angiopoietin-2. This is of interest as the dynamic balance in vessel regression and tumor growth has already been examined in a rat glioma model ${ }^{205}$. Two key players in this model are angiopoietins (Ang) and VEGF. Co-expression and increase in both VEGF and Angiopoietin-2 are associated with blood vessel proliferation. According to the authors, if there is overexpression of one of these players, there is vessel destabilization and regression. Other work has already demonstrated that concomitant expression of VEGF and Angiopoietin-2 resulted in increased microvessel density in solid tumors and cerebral angiogenesis ${ }^{204,208}$. The co-expression of these angiogenic factors contributes to the induction of microvessel sprouting in vascular networks ${ }^{209}$. Prior studies have examined the role of VEGF in Rb xenografts injected with Y79 cells and shown modest reductions in tumor burden ${ }^{166}$. However, this has failed to translate clinically. Collectively, our results show destabilization of angiogenic components, VEGF for Weri-1 and Angiopoietin-2 for Y79 Rb cells.

TGF- $\beta 1$ is a pleiotropic cytokine suggested to be the main inducer of tumor epithelial-to-mesenchymal (EMT) transition and to facilitate invasion by suppressing the host immune system ${ }^{210-212}$. In this study we found TGF- $\beta 1$ to be significantly reduced after MMP2I treatment in both Y79 and Weri-1 cells. Work done in the MCF10A breast cancer line highlighted the role of this cytokine in the upregulation of MMP-2 and MMP9; it is also known that these MMPs participate in growth factor cleavage for further cytokine release $^{213}$. TGF- $\beta$ is the subject of other ongoing studies as it was demonstrated to be localized in proximity to tumor vasculature and to promote drug resistance ${ }^{214}$ and has been implicated in $\mathrm{Rb}$ vitreous seeding ${ }^{215}$.

While this is the first study in $\mathrm{Rb}$ to examine MMP function and potential therapeutic value, there are limitations to the work presented above. For one, the in vitro approach fails to illustrate the complexity of in vivo tumors. The specific assays used in this work are classical assays that do not highlight the complex physiological processes they are meant to represent. Metastasis is a multi-faceted operation involving key molecular and genetic changes that cannot be captured in vitro. We address this complexity in future chapters by the development of in vitro assays which mimic the physiology of tumors in vivo in a three-dimensional (3D) manner. Another major limitation is the inhibitors used in these studies. We used two readily available MMPIs: ARP100 (MMP-2 inhibitor) and AG-L-66085 (MMP-9 inhibitor) that have failed to 
translate clinically, despite their heavy use in the understanding of MMP biology in vitro. While MMP inhibitors have yet to achieve widespread use in the clinic, a new generation of more targeted, safer inhibitors are emerging that may prove useful in targeting $\mathrm{Rb}$.

Future experiments should utilize an in vivo approach to understand more completely the roles MMPs play in $\mathrm{Rb}$ tumor metastasis. The work published here supports the hypothesis of MMP-2 and MMP-9 promoting two primary hallmarks of the metastatic process: invasion and migration. Future experiments to further support this hypothesis should be conducted in vivo using orthotopic xenografts of Y79 cells that will allow more mechanistic and physiologically-relevant insight in how Rb metastasizes. Stable RNA interference of either $M M P 2$ or $M M P 9$ in Y79 cells via shRNA should be utilized to determine if disruption of $M M P 2$ or $M M P 9$ mRNA could decrease or prevent metastatic disease in an orthotopic xenograft model. After qPCR assessment to determine efficacy of the RNA interference histopathological analyses should examine the extent of metastatic disease and progression ${ }^{37}$. We predict a reduction in the development of metastases in shRNA-treated animals. A potential caveat is that we do not understand how MMP knockdown may affect the implantation of cells after inoculation to develop the xenograft. Therefore, evaluation of the shRNA has to be done after inoculation of $\mathrm{Rb}$ cells. A more recent alternative to in vivo knockdown is the use of oligonucleotides and aptamers. Still, in vivo results would provide valuable insight into the future of MMP inhibitors for $\mathrm{Rb}$. These studies also open new avenues of research to develop more selective, safer MMP inhibitors based on RNA interference that can address the toxicities and lack of specificity associated with the previous generation of MMP-targeted small molecule inhibitors.

\section{Conclusion}

Our work reveals differences in several intrinsic properties associated with tumor progression in two cell lines representing the metastatic and non-metastatic forms of $\mathrm{Rb}$, Y79 and Weri-1. Based on our findings we developed a working model shown in Figure 2-7. In addition to the intrinsic differences in Y79 and Weri-1, MMP-2 and MMP-9 play different roles in these cells. MMP-2 and MMP-9 activity stimulate Rb cell migration in Y79 and contribute to cell viability in Weri-1 cells. Furthermore, MMP-9 activity plays a role in Angiopoietin-2 production in Y79. In contrast, MMP-2 and MMP-9 play additional roles in Weri-1 cells. More work is needed to follow up on these promising results. Taken together, we provide a comprehensive in vitro analysis of MMP-2 and MMP-9 activity in Rb in several checkpoints that are deregulated in cancer, including cell cycle, secretion of key immuno- and angiogenic modulators, viability, invasion, and migration. Our findings provide initial mechanistic insights into the benefits of potential MMP adjunct therapy in $\mathrm{Rb}$ patients. 


\section{A Y79, metastatic model}

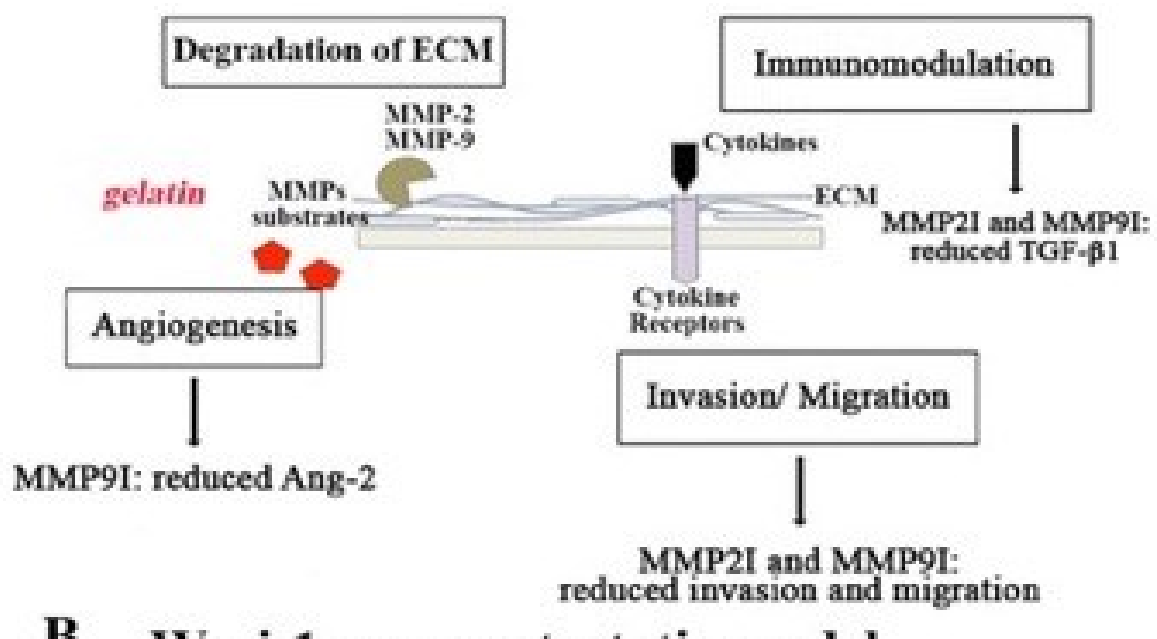

B Weri-1, non-metastatic model

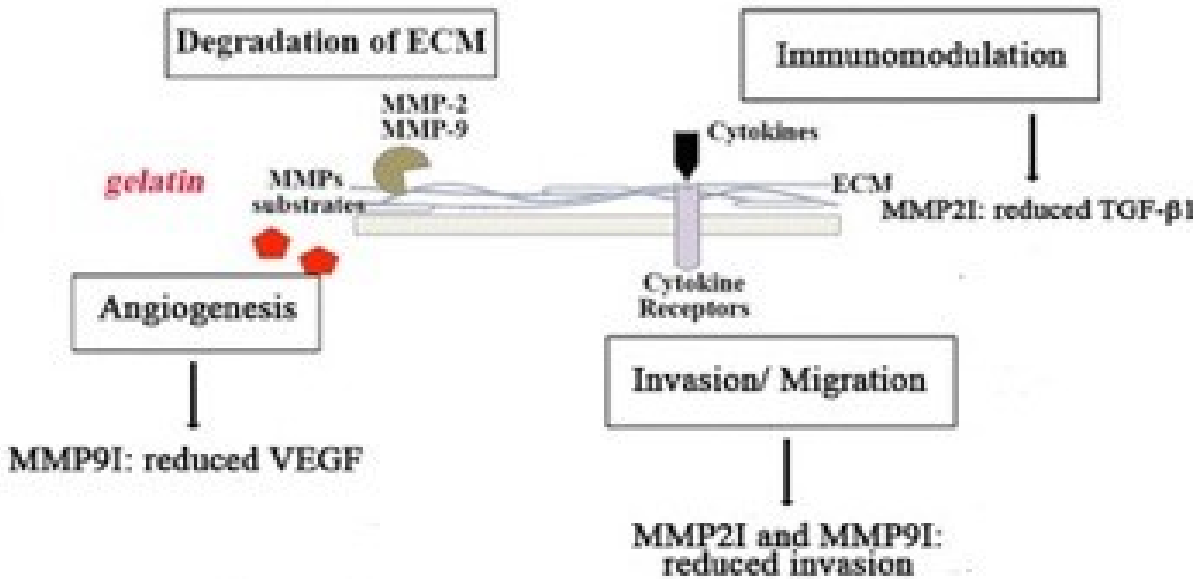

Figure 2-7. Working model of the roles of MMP-2 and MMP-9 in retinoblastoma cells.

Y79 and Weri-1 cells represent the metastatic and the non-metastatic model for Rb, respectively. Our work shows differences in viability, migration and angiogenicassociated responses in Rb cells after inhibition of MMP-2 and MMP-9. (A) Y79 cells showed a profound defect in migration and invasion along with and a significant reduction in Angiopoietin-2 and TGF- $\beta 1$ proteins. These results highlight Y79's migratory and invasive potential, which may be dependent upon MMPs. (B) Analyses of Weri-1 cells show MMP-2 and MMP-9 are involved in multiple processes, including viability of cells and VEGF, as well as TGF- $\beta 1$ production. Modified with permission from BMC Cancer. Webb AH, Gao BT, Goldsmith ZK, et al. Inhibition of MMP-2 and MMP-9 decreases cellular migration, and angiogenesis in in vitro models of retinoblastoma. BMC Cancer. 2017;17(1):434. Copyright (June 20, 2017), with permission through Creative Common CC-BY. 


\section{CHAPTER 3. RESULTS II: TARGETING THE PLATELET-DERIVED GROWTH FACTOR-BETA STIMULATORY CIRCUITRY TO CONTROL RETINOBLASTOMA SEEDS ${ }^{2}$}

\section{Introduction}

While metastatic $\mathrm{Rb}$ represents a clinical challenge and carries a dismal prognosis, $\mathrm{Rb}$ is commonly detected before it can disseminate to the brain or other distant organs. However, ocular salvage or preservation of the globe, remains very difficult; quite often enucleation is determined to be the best course of treatment. Even if enucleation is not initially performed, patients receiving chemotherapy are left partially or completely blind and will undergo enucleation in the event of recurrent $\mathrm{Rb}$. The greatest impediment to raising ocular salvage rates is the presence of tiny pockets of tumors within the vitreous, called vitreous seeds (or seeding). These seeds are also thought to be the primary cause of $\mathrm{Rb}$ recurrence. Although some vitreous seeds are responsive to treatment, the majority of cases are refractory and the mechanisms controlling their refractory status are unclear ${ }^{217}$. Both external beam radiotherapy (EBRT) and chemotherapy, regardless of route of delivery (systemic, subconjunctival, intraarterial or intravitreal), have failed to provide ocular salvage rates of greater than $70 \%$ for eyes with vitreous seeds at diagnosis ${ }^{218-221}$. Overall, patients that present with vitreous seeding have a poorer prognosis for ocular salvage ${ }^{180,218,221-223}$. While the mechanism controlling refractory or recurrent vitreous disease is unclear, it is likely related to a unique property of $\mathrm{Rb}$ cells that allows them to adapt and propagate in semi-solid or liquid microenvironments, such as the vitreous and sub-retinal compartments ${ }^{100}$. Recent literature suggests these $\mathrm{Rb}$ cells remain in a semi-dormant state as vitreous seeds for long periods of time and show reduced proliferative capacity and metabolism ${ }^{224}$.

Understanding the tumor microenvironment is an important step in achieving overall treatment goals for $\mathrm{Rb}$, including patient survival, globe salvage and vision preservation ${ }^{217,224}$. The microenvironment of the vitreous has not been well characterized, in particular immunological studies on the interaction of the vitreous microenvironment and $\mathrm{Rb}$ are lacking. The vitreous body is a translucent medium in the eye composed of a liquid (99\%) and solid (1\%) phase. Recent research has identified the presence of some inflammatory, mitogenic, and immunosuppressive mediators in the vitreous ${ }^{109-111}$. These soluble proteins in the vitreous include growth factors, which promote homeostasis as well as pathologic processes, most notably diabetic retinopathy and proliferative vitreous retinopathy ${ }^{110,149,225,226}$. These growth factors have been investigated for potential therapeutics in a number of diseases including age-related macular degeneration $(\mathrm{AMD})^{227}$, diabetic retinopathy (DR), and proliferative vitreoretinopathy (PVR) ${ }^{154}$.

\footnotetext{
${ }^{2}$ Adapted with permission. Goldsmith ZK, Coppess W, Irvine AS, et al. Targeting the Platelet-Derived Growth Factor-beta Stimulatory Circuitry to Control Retinoblastoma Seeds. Investigative Ophthalmology \& Visual Science. 2018;59(11):4486-4495. (C) Association for Research in Vision and Ophthalmology.
} 
Platelet derived growth factor (PDGF) has been identified as one such key regulator ${ }^{149,228}$ within the vitreous and has recently been targeted in treatment of DR and PVR ${ }^{155}$.

PDGF signaling through its receptors has been shown to contribute to different pathological processes including tumorigenesis ${ }^{229-231}$ and angiogenesis ${ }^{229,230,232-234}$. Cellular infiltration, including tumor cells, macrophages, and platelets, may produce and secrete PDGF in the vitreous microenvironment that could provide paracrine and autocrine stimulation and mitogenic activity to $\mathrm{Rb}$ cells via the platelet-derived growth factor receptors (PDGFRs). We investigated the PDGFR $\beta$ signaling pathway to better understand and potentially target $\mathrm{Rb}$ vitreous seed growth. We found that reducing PDGFR $\beta$ signaling in $\mathrm{Rb}$ tumor cells in vitro reduces tumor cell growth and survival via the MDM2 and NFאB signaling pathways.

\section{Materials and Methods}

\section{Ethics statement}

The $\mathrm{Rb}$ xenografts used in this chapter were developed using human tissue derived from $\mathrm{Rb}$ patients. Those experiments involving human subjects were approved by the Institutional Review Board (IRB) at St. Jude Children's Research Hospital (SJCRH) and The University of Tennessee Health Science Center (UTHSC); informed consent obtained when needed. In those experiments using tissue from murine studies, the Institutional Animal Care and Use Committee (IACUC) at SJCRH approved the animal experiments. All protocols followed the Association for Research in Vision and Ophthalmology (ARVO) Statements for the use of animals in vision research, in addition to the guidelines for laboratory animal experiments.

\section{Cell lines and growth media}

The human Retinoblastoma $(\mathrm{Rb})$ cell lines Y79 $\left(\mathrm{ATCC}^{\circledR} \mathrm{HTB}-18^{\mathrm{TM}}\right)^{79}$ and WeriRb- ${ }^{80}$ (ATCC ${ }^{\circledR}$ HTB-169 ${ }^{\mathrm{TM}}$ ) were purchased from the American Type Culture Collection (Manassas, VA, USA) and cultured in Rb media. This media contains RPMI1640 supplemented with L-glutamine (Gibco by Invitrogen, Carlsbad, CA, USA), 20\% FBS (Gibco by Invitrogen), and 1\% penicillin/ streptomycin (Gibco by Invitrogen). Cells were kept in a humidified incubator at $37^{\circ} \mathrm{C} / 5 \% \mathrm{CO}_{2}$. Cells were passaged every 3 days to keep the cells from reaching over $80 \%$ confluency.

\section{Orthotopic xenografts}

After receiving the appropriate consent tumor samples from enucleated eyes were isolated from a diverse population of patients treated at St. Jude Children's Research Hospital (Memphis, TN). These specimens were placed in RPMI media before being 
processed for engraftment. To prepare for inoculation, tumor samples were first dissociated in a solution of trypsin and $\mathrm{PBS}$ at $37^{\circ} \mathrm{C}$. Resulting cell suspension was washed and counted. Tumor cells were resuspended in RPMI with $10 \%$ fetal calf serum at approximately 10,000 cells $/ \mu \mathrm{L}$. A volume of $5 \mu \mathrm{L}$ (approximately 50,000 cells) were injected into each eye of anesthetized SCID (B6.CB17-Prkd ${ }^{\text {scid }}$ /SzJ; Jackson Labs) mice. Tumor progression was then monitored with a digital retinal camera and intraocular pressure was regularly measured ${ }^{88}$.

\section{Vitreous samples}

The University of Tennessee Health Science Center and St. Jude Children's Research Hospital Institutional Review Board (IRB) approved the present study. Informed consent was obtained in all cases where vitreous was harvested at the time of patient enucleation, an optional research objective that is part of an on-going prospective clinical trial (NCT01783535) This is in full compliance with and adheres to the tenets of the Declaration of Helsinki and the ARVO statement on human subjects. Vitreous from eyes with $\mathrm{Rb}$ was collected at the time of enucleation. Eyes were trephined immediately upon removal without disruption of underlying structures important for diagnostic and staging purposes. Tumor was harvested for genetics and biology and vitreous was aspirated using an 18-gauge needle and $3 \mathrm{~mL}$ syringe. Vitreous from healthy controls were purchased from BioreclamationIVT (Baltimore, MD, USA). Samples were centrifuged to collect supernatants followed by sonication. Samples were aliquoted and stored at $-20^{\circ} \mathrm{C}$.

\section{Reagents}

Phosphate-buffered saline (PBS) was purchased from Gibco by Invitrogen (Thermo Scientific, Bridgewater, NJ, USA). Gleevec ${ }^{\mathbb{B}}$ was purchased in its generic chemical form, imatinib mesylate (IM, PubChem ID: 123596), from Sigma-Aldrich (Saint Louis, MO, USA). Lyophilized reagent was dissolved in deionized, distilled water $\left(\mathrm{ddH}_{2} \mathrm{O}\right)$ to $10 \mathrm{mM}$, aliquoted, and stored at $-20^{\circ} \mathrm{C}$. Lyophilized recombinant human PDGF-BB $(10 \mu \mathrm{g})$ was purchased from Invitrogen (Thermo Scientific), dissolved in acetic acid, aliquoted, and stored at $-20^{\circ} \mathrm{C}$.

\section{Immunohistochemistry (IHC)}

Enucleated eyes from $\mathrm{Rb}$ patients treated at SJCRH were fixed, embedded in paraffin and serially sectioned. Samples were probed with anti-human PDGFR $\beta$ (\#16868, Abcam, Cambridge, MA, USA), for non-phosphorylated PDGFR $\beta$, and anti-human pPDGFR $\beta$ (\#sc-339, Santa Cruz Biotechnology, Inc., Dallas, TX, USA) to label active protein for IHC. Enucleated eyes from murine xenografts were fixed, embedded in paraffin and serially cut at $4 \mu \mathrm{m}$ thickness using a Leica rotary microtome before sections 
were mounted on gelatin-coated histologic slides ${ }^{88}$. For more information, please see Table A-2.

\section{MTS cell proliferation assay}

Cell proliferation, as a measure of cell viability, was investigated using the CellTiter $96^{\circledR}$ AQ ueous One Solution Cell Proliferation Assay reagent (Promega, Madison, WI, USA). Briefly, Y79 cells were plated in a 96-well plate at a density of $5.0 \times 10^{4}$ cells per well. Cells were cultured under different conditions, including Rb media (untreated), $10 \mathrm{ng} / \mathrm{mL}$ recombinant human PDGF-BB (rhPDGF-BB), $10 \mu \mathrm{M}$ imatinib mesylate (IM), or the combination of rhPDGF-BB and IM. Plates were incubated for 0-, 48-, 72-, 96-, and $120 \mathrm{~h}$. Proliferation was determined through colorimetric change of absorbance. The absorbance values were calculated from a spectrophotometric microplate reader (BioTek $\mu$ Quant, BioTek Instruments, USA) using 490nm as reading wavelength and reference wavelength at $630 \mathrm{~nm}$. Final values were calculated by subtracting media-only blanks from experimental samples. Assays were conducted in quadruplicate.

\section{Protein assays}

PDGF-AB / PDGF-BB ELISA. Vitreous samples were diluted 1:10 with Assay Buffer from the Invitrogen Human PDGF-BB Platinum ELISA Kit (Invitrogen, Thermo Scientific). Diluted samples were placed in either a pre- human PDGF-AB antibodycoated microwell or a human PDGF-BB antibody-coated microwell plate in triplicate, along with reconstituted standards in a serial fold dilution following the manufacturer's instructions. After incubation for $2 \mathrm{~h}$ at $\mathrm{RT}$, the provided biotin-conjugated anti-human PDGF-AB or PDGF-BB antibody was added. After several washes, streptavidin-HRP was added followed by incubation with the TMB substrate solution (tetremethylbenzidine). Absorbance values were calculated from a spectrophotometric microplate reader (BioTek $\mu$ Quant) using both $450 \mathrm{~nm}$ and $550 \mathrm{~nm}$ as reading wavelengths. Averages and standard error measurements of the cytokines in both the samples and controls were calculated and plotted in Prism Graph Pad.

Western blot analyses. Y79 cells were treated using the conditions described above: $\mathrm{Rb}$ media with $10 \mathrm{ng} / \mathrm{mL}$ recombinant human PDGF-BB (rhPDGF-BB), $10 \mu \mathrm{M}$ imatinib mesylate (IM), or the combination of rhPDGF-BB and IM. Cells in complete $\mathrm{Rb}$ media (untreated) were used as baseline controls. Cells were then lysed using cold RIPA buffer containing both Halt ${ }^{\mathrm{TM}}$ protease and phosphatase inhibitors (Thermo Scientific). Cell lysates were centrifuged at 2500 RPM x 5min to shear DNA. Protein from whole cell lysate of $50 \mu \mathrm{g}$ was separated on Bolt ${ }^{\circledR} 4-12 \%$ Bis-Tris Plus Gels (Thermo Scientific) and transferred to an Amersham Hybond polyvinylidene difluoride (PVDF) membrane (GE Health Care Life Sciences, Pittsburgh, PA, USA). Membranes were blocked with $5 \%$ BSA in Pierce ${ }^{\mathrm{TM}}$ Tris Buffered Saline Tween-20 (TBST, Thermo Scientific) for $1 \mathrm{~h}$ followed by incubation with primary antibodies overnight at $4^{\circ} \mathrm{C}$. Thereafter, HRP-linked secondary antibodies were added and incubated at RT for $1 \mathrm{hr}$. All experiments were 
conducted in triplicate. Primary antibodies used were: polyclonal rabbit anti-PDGFR $\beta$ (Santa Cruz Biotechnology, sc-432; 1:100), monoclonal mouse anti-phosphorylated PDGFR $\beta$ (Santa Cruz Biotechnology, sc-373805; 1:100), monoclonal mouse anti-VEGF (Santa Cruz Biotechnology, sc-53462; 1:200), monoclonal mouse anti-Flk-1 (aka VEGFR2, Santa Cruz Biotechnology, sc-6251; 1:200), polyclonal rabbit anti-p-Flk-1 (aka p-VEGFR2, Santa Cruz Biotechnology, sc-101821; 1:100), monoclonal mouse antiMDM2 (Santa Cruz Biotechnology, sc-965; 1:200), monoclonal mouse antiphosphorylated MDM2 (Santa Cruz Biotechnology, sc-53368; 1:200), polyclonal rabbit anti-AKT (Cell Signaling Technology, \#9272; 1:1000), monoclonal rabbit antiphosphorylated AKT (Cell Signaling Technology, \#4058; 1:1000), monoclonal rabbit anti-BCL-2 (Cell Signaling Technology, \#2870; 1:1000), monoclonal rabbit anti-GAPDH (Cell Signaling Technology, \#8884; 1:1000), Secondary antibodies used were: antimouse IgG HRP-linked Antibody (Cell Signaling Technology, \#7076; 1:1000) and antirabbit IgG HRP-linked Antibody (Cell Signaling Technology, \#7074; 1:1000). Endogenous control used was either $\beta$-actin or GAPDH (both from Cell Signaling Technology). Signal was detected using SuperSignal ${ }^{\mathrm{TM}}$ West Pico Chemiluminescent Substrate (Thermo Scientific). Densitometry analysis was done using Kodak Molecular Imager, as described in the previous chapter ${ }^{195-197}$ For more information, please see Table A-2.

Cleaved caspase-3 ELISA. A commercially available Caspase-3 (active) ELISA kit (Invitrogen, Thermo Scientific) was used to assess apoptotic activity. Briefly, $1.0 \mathrm{x}$ $10^{6}$ Y79 cells were cultured in complete Rb media or Rb media supplemented with either $10 \mathrm{ng} / \mathrm{mL}$ recombinant human PDGF-BB (rhPDGF-BB), $10 \mu \mathrm{M}$ imatinib mesylate (IM), or the combination of rhPDGF-BB and IM for 48h. Cells were collected, and protein was extracted using Cell Extraction Buffer (Invitrogen) with protease and phosphatase inhibitors (Thermo Scientific) before being quantified by Pierce BCA Protein Assay Kit. The ELISA was completed according to manufacturer's instructions: $30 \mu \mathrm{g}$ of total extracted protein was incubated in a microplate well in triplicate at room temperature for $2 \mathrm{~h}$. Samples were washed followed by addition of $100 \mu \mathrm{L}$ detection antibody (caspase-3) for $1 \mathrm{~h}$ at RT. This antibody was removed and replaced with anti-rabbit IgG HRP antibody for 30min at RT. Antibody was removed and Stabilized Chromogen was added to develop blue color for approximately $30 \mathrm{~min}$ before being stopped by addition of $100 \mu \mathrm{L}$ stop solution and solution turned yellow. Absorbance values were calculated from a spectrophotometric microplate reader (BioTek $\mu$ Quant) using 450nm as the reading wavelength. Averages and standard deviations of the levels of active Caspase- 3 in both the samples and controls were calculated and plotted in Prism Graph Pad.

Bicinchoninic acid (BCA) protein concentration assay. Albumin standards were generated ranging from $2000 \mu \mathrm{g} / \mathrm{mL}$ to $25 \mu \mathrm{g} / \mathrm{mL}, 0 \mu \mathrm{g} / \mathrm{mL}$ and $20 \mu \mathrm{L}$ of prepared standards were plated on a 96-well microplate. A volume of $2 \mu \mathrm{L}$ of fresh lysate was added (in triplicate) followed by addition of $200 \mu \mathrm{L}$ BCA working solution $(50: 1$, Reagent $\mathrm{A}: \mathrm{B})$. Plates was mixed gently on the benchtop prior to incubation at $37^{\circ} \mathrm{C}$ for $30 \mathrm{~min}$. Plate was then read on a spectrophotometric microplate reader (BioTek $\mu$ Quant, BioTek Instruments, USA) at $562 \mathrm{~nm}$. Using linear fit, protein concentrations were generated in $\mu \mathrm{g} / \mu \mathrm{L}$. 


\section{Flow cytometry studies}

Annexin V and PI labeling. To measure apoptotic activity of Y79 cells, Annexin-V (A5) and propidium iodide (PI) were used. After treatment as described above, cells were harvested at 0-, 24-, 48-, 72-, and 96h time points and labeled with $10 \mu \mathrm{L}$ A5-PerCP-Cy5.5 and 5 $\mu \mathrm{L}$ PI (BioLegend) and incubated away from light at RT for $15 \mathrm{~min}$. An additional $200 \mu \mathrm{L}$ of Annexin V Binding Buffer were added prior to analysis to have a final volume of $400 \mu \mathrm{L}$. Data was acquired using a $\mathrm{BD}^{\mathrm{TM}}$ LSR II Cytometer (BD Biosciences) using BD FACSDiva ${ }^{\mathrm{TM}}$ software and analysis performed using FlowJo v.X.0.0.8 (FlowJo, LLC, Tree Star, Ashland, OR, USA). Samples were analyzed in triplicate.

Zombie Aqua ${ }^{\text {TM }}$ labeling. To assess live versus dead status, we used Zombie Aqua $^{\mathrm{TM}}$ (BioLegend, \#423102), an amine-reactive fluorescent dye permeant to cells with compromised membranes. Lyophilized dye was reconstituted in $100 \mu \mathrm{L}$ of DMSO and used at a dilution of 1:100 for up to $5 \times 10^{6}$ cells in PBS. Cells were incubated for $20 \mathrm{~min}$ at RT, covered from light, prior to washing twice with PBS/1\% FBS. Data acquired in a $\mathrm{BD}^{\mathrm{TM}}$ LSR II Cytometer (BD Biosciences) using BD FACSDiva ${ }^{\mathrm{TM}}$ software. Analysis performed using FlowJo v.X.0.0.8 (Tree Star).

Assessment of expression of PDGFR $\beta$. Y79 Rb cells cultured in $\mathrm{Rb}$ media or $\mathrm{Rb}$ media including 10ng/mL recombinant human PDGF-BB (rhPDGF-BB), 10 $\mu \mathrm{M}$ imatinib mesylate (IM), or the combination of rhPDGF-BB and IM were labeled with polyclonal rabbit anti-PDGFR $\beta$ (Santa Cruz Biotechnology, sc-432; 1:50) on ice for 30min followed by incubation with an anti-rabbit Alexa Fluor ${ }^{\mathbb{B}} 647$ (Thermo Scientific, 1:50) secondary antibody for $30 \mathrm{~min}$. Cells were fixed with $2 \%$ paraformaldehyde for $20 \mathrm{~min}$ prior to analysis. Data acquisition was done in a ZE5 Cell Analyzer (aka YETI), from Propel Labs (Fort Collins, CO, USA). Analysis was done using FlowJo software v.X.0.0.8 (Tree Star). For more information, please see Table A-2.

p65 NFkB Nuclear Translocation by imaging flow cytometry. Y79 Rb cells cultured $\mathrm{Rb}$ media or $\mathrm{Rb}$ media including $10 \mathrm{ng} / \mathrm{mL}$ recombinant human $\mathrm{PDGF}-\mathrm{BB}$ (rhPDGF-BB), $10 \mu \mathrm{M}$ imatinib mesylate (IM), or the combination of rhPDGF-BB and IM. Collected cells were transferred into low binding micro-centrifuge tubes, washed in PBS and fixed in 2\% paraformaldehyde prior to permeabilization in $0.01 \%$ Triton $\mathrm{X}-100$. Samples were blocked with PBS/1\% FBS before addition of a monoclonal rabbit anti-p65 (Cell Signaling Technology, \#8242, 1:100) antibody for 1h in ice. Following primary antibody labeling, cells were washed with PBS and an anti-rabbit Alexa Fluor ${ }^{\circledR} 488$ (Thermo Scientific, 1:100) secondary antibody was added for $1 \mathrm{~h}$. Nuclei were labeled

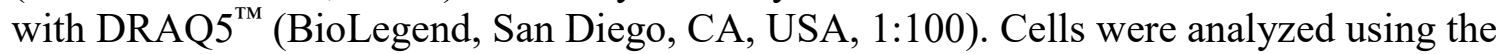
Amnis FlowSight ${ }^{\circledR}$ Imaging Cytometer (EMD Millipore, Amnis Corporation, Seattle, WA, USA). Data was analyzed using the "Nuclear Translocation" feature in IDEAS ${ }^{\circledR}$ software (EMD Millipore, Amnis Corporation). Mean fluorescent intensity (MFI) was measured to investigate levels of p65 as well as percentage of cells with p65 translocation 
to the nucleus. We used the $488 \mathrm{~nm}, 642 \mathrm{~nm}$, and $785 \mathrm{~nm}$ excitation lasers. The fluorescence controls were single labeled cells acquired with both $488 \mathrm{~nm}$ and $642 \mathrm{~nm}$ lasers while ensuring the Side Scatter (SSC, 785nm) and Bright Field (BF) channels were OFF. We collected a minimum of 10,000 events (cells) from each sample; experiments done in triplicate. For more information, please see Table A-2.

\section{Invasion assay}

CytoSelect ${ }^{\mathrm{TM}}$ Cell Invasion Assay kit was purchased from Cell BioLabs, Inc (San Diego, CA). We used the $8 \mu \mathrm{m}$ pore polycarbonate membrane coated with basement membrane matrix solution. Rb cell suspension (serum free media) was placed in the upper chamber to determine the invasion capacity of the cells after degradation of the matrix membrane proteins $6 \mathrm{~h}$ post culture. Invasive cells were stained using $400 \mu \mathrm{L}$ of the included Cell Stain Solution and incubated at RT for 10min before being washed with distilled water and quantified with a light microscope under $100 \mathrm{X}$ total magnification (lens 2.5X, objective 40X), with 4 individual fields per insert. Inserts were placed into wells containing $200 \mu \mathrm{L}$ of Extraction Solution followed by $10 \mathrm{~min}$ incubation at RT on an orbital shaker. Quantitation of cells measured at OD 560nm using spectrophotometer.

\section{Three-dimensional cell culture system}

Y79 cells were harvested and labeled with the carboxyfluorescein succinimidyl ester (CFSE) Cell Division Tracker Kit (BioLegend). Briefly, cells were washed in PBS prior to addition of $1 \mu \mathrm{M}$ CFSE per $\mathrm{mL}$ of cell suspension. Cells were incubated for $5 \mathrm{~min}$ at $37^{\circ} \mathrm{C}$ prior to quenching on ice for $5 \mathrm{~min}$ with $20 \mathrm{~mL}$ cold complete media. After quenching, cells were washed twice in cold media. CFSE-labeled cells were mixed with nanoshuttles using $1 \mu \mathrm{L}$ of nanoshuttles (n3D Biosciences, Houston, TX, USA) per 10,000 cells. These cells were incubated overnight at $37^{\circ} \mathrm{C} / 5 \% \mathrm{CO}_{2}$. The next day, cells with nanoshuttles were magnetized for $30 \mathrm{~min}$ using the 6 -well concentrating drive (n3D Biosciences, Houston, TX, USA) and plated at $2.5 \times 10^{5}$ cells per well of a 6 -well plate with a $2 \mathrm{~mL}$ final volume. Cells were re-magnetized for $30 \mathrm{~min}$ using the 6 -well levitating drive (n3D Biosciences, Houston, TX, USA) before the magnet was removed. The plate was placed in a NanoDock Station for $48 \mathrm{~h}$ in the incubator. Data images were recorded every hour using an iPod (Apple) and a magnifying glass on top of the plate. Data analysis was performed using the NIH ImageJ Software (imagej.nih.gov/ij/). The images were converted to black and white using the Image J Software Color Thresholding tool. Measurements were taken of the growth in each well for $48 \mathrm{~h}$. Color threshold and brightness were kept constant across all images. Following 48h, spheroids were fixed with 4\% paraformaldehyde for $4 \mathrm{~h}$ at RT and then washed with PBS (magnet was placed under plate during washes to maintain spheroid). Labeling was done by adding $2 \mathrm{~mL}$ of Antigen Retrieval Solution and incubated at $8^{\circ} \mathrm{C}$ for $20 \mathrm{~min}$ before being washed accordingly. We added $500 \mu \mathrm{L}$ of $0.01 \%$ Triton X-100 permeabilization buffer for $15 \mathrm{~min}$ at RT. To prevent non-specific binding, $1 \mathrm{~mL}$ of $\mathrm{PBS} / 10 \% \mathrm{FBS}$ was added and incubated for $1 \mathrm{~h}$ at RT. Blocking solution was aspirated off and primary antibody was added for 
overnight incubation at $4^{\circ} \mathrm{C}$. Primary antibodies included: monoclonal rabbit antiphosphorylated VEGFR2 (Cell Signaling Technology, \#3770; 1:100) and polyclonal rabbit anti-PDGFR $\beta$ (Santa Cruz Biotechnology, sc-432; 1:100). Alexa Fluor ${ }^{\circledR} 647$ donkey anti-rabbit IgG (Invitrogen, A31573; 1:100) secondary antibody was added and incubated for $2 \mathrm{~h}$ at RT followed by adequate washing. Samples were allowed to air dry for $1 \mathrm{~h}$ before being imaged on a Nikon $\mathrm{C} 1$ confocal microscope. All microscope settings, including laser levels, were held constant and images were collected in identical methodology to allow for relative comparisons of signal intensity within and between experiments.

\section{qPCR analyses}

RNA isolation. RNA from $2.5 \times 10^{6} \mathrm{Rb}$ cells was extracted following the Qiagen ${ }^{\circledR}$ miRNeasy Mini Kit (Qiagen, Valencia, CA) manufacturer's recommendations. $\mathrm{Rb}$ cells were harvested and centrifuged to remove all traces of cell culture medium. Cell pellets were lysed and homogenized prior to addition of chloroform. Samples were shaken vigorously for $15 \mathrm{~s}$ prior to centrifugation for $2 \mathrm{~min}$ at full speed. The upper colorless phase was transferred to a clean tube after centrifugation followed by a series of alcohol precipitations to provide ideal binding conditions. The lysate was loaded onto an RNeasy spin column with a silica membrane to bind the RNA and washed away contaminants. Residual DNA was removed by on-column DNase digestion. The concentrated RNA was eluted in $15 \mu \mathrm{L}$ of RNAse free water. RNA concentration was assessed by analysis on Nanodrop 2000 Spectophotometer (ThermoFisher Scientific) and purity was assessed by verifying absorbance ratios of 260nm and 280nm (A260/A280). Samples with absorbance ratios between 1.8 and 2.0 were considered free of contamination.

cDNA synthesis. Synthesis of cDNA was performed using the SuperScript ${ }^{\circledR}$ VILO $^{\text {TM }}$ cDNA Synthesis Kit (Life Technologies, Grand Island, NY). Following manufacturer's directions, we used 100ng of isolated RNA (see section above) and combined them with $4 \mu \mathrm{L} 5 \mathrm{X}$ VILO Reaction Buffer and $2 \mu \mathrm{L} 10 \mathrm{X}$ SuperScript Enzyme Mix. Final volume per sample was $20 \mu \mathrm{L}$ using DEPC-treated water when necessary. Per manufacturer's directions, cDNA synthesis reaction was done at $25^{\circ} \mathrm{C}$ for $10 \mathrm{~min}$ followed by $42^{\circ} \mathrm{C}$ incubation for $60 \mathrm{~min}$ with reaction termination at $85^{\circ} \mathrm{C}$ for $5 \mathrm{~min}$.

Pre-amplification and PCR. The synthesized cDNA was preamplified with TaqMan ${ }^{\circledR}$ PreAmp Master Mix to improve detection sensitivity and optimize the use of limited material. The following gene expression assays were used (Thermo Scientific): PDGFRA (Hs009998018_m1), PDGFRB (Hs01019589_m1), PDGFA (Hs00234994_m1), PDGFB (Hs_00966522_m1), MDM2 (Hs00540450_m1), MDM4 (Hs00910358_m1), VEGFA (Hs00900055_m1), FLT1 (aka VEGFR2, Hs01052961_m1) HPRT1 (Hs02800695_m1) all from Life Technologies (Grand Island, NY). Material was preamplified using $10 \mu \mathrm{L} 2 \mathrm{X}$ TaqMan ${ }^{\circledR}$ PreAmp Master Mix, $5 \mu \mathrm{L}$ pooled $0.2 \mathrm{X}$ TaqMan ${ }^{\circledR}$

primers, and $5 \mu \mathrm{L}$ cDNA sample for a final volume of $20 \mu \mathrm{L}^{192}$. Preamplification reactions were conducted according to manufacturer's guidelines with a $10 \mathrm{~min}$ enzymatic 
activation step at $95^{\circ} \mathrm{C}$ followed by 14 cycles of denaturing $\left(95^{\circ} \mathrm{C}\right.$ for $\left.15 \mathrm{~s}\right)$ and annealing $\left(60^{\circ} \mathrm{C}\right.$ for $\left.4 \mathrm{~min}\right)$. Following 14 cycles, enzyme was inactivated at $99^{\circ} \mathrm{C}$ for $10 \mathrm{~min}$ and reactions were held at $4^{\circ} \mathrm{C}$ until use. Preamplification utilized the same primers analyzed to use minimal amounts of material while increasing sensitivity of detection. The reaction was kept at $-20^{\circ} \mathrm{C}$ until ready to use. A final volume of $10 \mu \mathrm{L}$ was loaded into each well after combination of $5 \mu \mathrm{LTaqMan}^{\circledR}$ Universal Master Mix, $2.5 \mu \mathrm{L}$ cDNA, $0.5 \mu \mathrm{Lprimers}$ and $2.0 \mu \mathrm{L}$ Nuclease-Free water. White, 96-well PCR plates were run using Roche ${ }^{\circledR}$ LightCycler 480 using TaqMan ${ }^{\circledR}$ protocol: reaction was initiated at $50^{\circ} \mathrm{C}$ for $2 \mathrm{~min}$ followed by $95^{\circ} \mathrm{C}$ for $10 \mathrm{~min}$ before $40 \mathrm{PCR}$ cycles were conducted at $95^{\circ} \mathrm{C}$ for $15 \mathrm{~s}$ and $60^{\circ} \mathrm{C}$ for $1 \mathrm{~min}$. Values greater than 35 were excluded due to low confidence. Data was analyzed using the Comparative $\mathrm{C}_{\mathrm{T}}\left(\Delta \Delta \mathrm{C}_{\mathrm{T}}\right)$ Method where $\Delta \mathrm{C}_{\mathrm{T}}$ is the difference between gene-of-interest (GOI) and housekeeping gene (HKG) and $\Delta \Delta \mathrm{C}_{\mathrm{T}}$ is the difference between experimental and untreated. Fold change is determined by 2 raised to the negative $\Delta \Delta \mathrm{CT}$ value $\left[2^{(-\Delta \Delta \mathrm{CT})}\right]^{192,193}$. For more information, please see Table A-1.

siRNA transfections. Y79 cells were plated overnight in 6-well plates at a final density of $3.0 \times 10^{5}$ cells per well in $2 \mathrm{~mL} \mathrm{Rb}$ media (without antibiotics). Lyophilized PDGFRB siRNA duplex (sc-29942, Santa Cruz Biotechnology) was diluted in nucleasefree water to a final concentration of $10 \mu \mathrm{M}$ following manufacturer's instructions. A total of $0.6 \mu \mathrm{g}$ of $P D G F R B$ siRNA was diluted in $100 \mu \mathrm{L}$ of siRNA Transfection Medium (Santa Cruz Biotechnology) per well (Solution A). In parallel, $6 \mu \mathrm{L}$ of siRNA Transfection Reagent were added into $100 \mu \mathrm{L}$ siRNA Transfection Medium (Solution B) per well. Solution A and solution B were combined and incubated at RT for 30min. Meanwhile, Y79 cells were harvested, washed in Transfection Medium and were resuspended in $800 \mu \mathrm{L}$ of siRNA Transfection Medium per well following addition of Solution $\mathrm{A}+\mathrm{B}$. Cells were incubated for $6 \mathrm{~h}$ at $37^{\circ} \mathrm{C} / 5 \% \mathrm{CO}_{2}$. At this point, $1 \mathrm{~mL}$ of RPMI / 20\% FBS was added and cells were incubated for $18 \mathrm{~h}$ prior to performing functional assays. As a control, we used a scramble sequence that is known to not target any specific oligonucleotides.

Statistical analyses. Data were analyzed using Prism 6 for Mac OS X (GraphPad Software, Inc., La Jolla, CA). Statistical significance determined using the Holm-Sidak method, with alpha $=5.000 \%$. Values where $p<0.05$ were considered significant.

\section{Results}

\section{Expression of the Platelet-Derived Growth Factor Receptor signaling network in retinoblastoma tumor and tumor-derived cell lines}

Proteomic analyses have identified over 600 proteins within the vitreous ${ }^{235}$. Some of these proteins are known to play significant roles in pathological conditions that including vitreal components, most notably: VEGF and PDGF in diabetic retinopathy (DR) and age-related macular degeneration (AMD). A common denominator of these ocular pathologies is the presence of PDGF. Additionally, a previous study reported 
limited success in targeting VEGF in $\mathrm{Rb}$, another abundant growth factor within the vitreous. We hypothesized PDGF within the vitreous, not VEGF, could sustain vitreous seeds via activation of the Platelet-Derived Growth Factor Receptor-beta (PDGFR $\beta$ ). To first test this hypothesis, we investigated the expression of PDGFR $\beta$ in primary human Rb samples from enucleated eyes by immunohistochemistry (IHC). Our results in Figure 3-1A-D measured low expression of the non-phosphorylated form of the PDGFR $\beta$ protein and high expression of the active or phosphorylated form of PDGFR $\beta$ ( $p$ PDGFR $\beta$ ) in ex vivo primary tumor tissue. Next, we analyzed samples by IHC from eyes of $\mathrm{Rb}$ patients with advanced intraocular diffuse disease vitreous seeds that were primarily enucleated. We measured high expression of the p-PDGFR $\beta$ (Figure 3-1E,F) compared to the non-phosphorylated PDGFR $\beta$. The human orthotopic xenograft for $\mathrm{Rb}$ has been already established as a comparable model to $\mathrm{Rb}$ disease with the molecular signatures of the primary tumors. This xenograft model is generated by engraftment of $\mathrm{Rb}$ cells derived from tissue of patients afflicted with $\mathrm{Rb}$ that are treated at St. Jude Children's Research Hospital. Harvested Rb cells are injected intravitreally into SCID mice to generate tumors that may be monitored both by retinal camera as well as tonometry to measure intraocular pressure for several weeks. Mice may then be sacrificed before enucleation, paraffin-embedding of tissue, cryo-sectioning, and immunolabeling. Details on the xenograft have been already published ${ }^{88}$. Using this system, we investigated the expression of PDGFR $\beta$ in samples from human patient-derived orthotopic xenografts (PDX). As shown in Figure 3-1G,H the IHC displayed low expression of the non-phosphorylated PDGFR $\beta$ compared to the p-PDGFR $\beta$. Taken together, we observed activity of the PDGFR $\beta$ signaling network in vivo.

Gene expression of the PDGFR network was evaluated by qPCR analyses to quantify mRNA expression of the most common members of the PDGF family. Examination of mRNA levels would also allow us to confirm our IHC results above (Figure 3-1G,H). To differentiate between human Rb cells and the murine microenvironment (host) by the species-specificity of the gene expression assays. We analyzed a cohort of paraffin embedded tissue samples from PDX in and measured higher PDGFRB mRNA expression compared to PDGFRA ((Figure 3-2, left) $23.0 \pm 10.0$ compared to $8.1 \pm 0.01$, mean $\pm \mathrm{SEM}$ ). $P D G F B$ mRNA was also measured at higher levels that $P D G F A$ in the PDX samples (3.2 \pm 2.0 compared to $1.8 \pm 2.0)$.

The Y79 and Weri-1 Rb cells are the most characterized $\mathrm{Rb}$ tumor-derived cell lines. Y79 is considered a metastatic and aggressive cell line while Weri-1 is considered the non-metastatic Rb model ${ }^{37}$. Y79 measured higher expression of PDGFRB compared to the PDGFRA mRNA ((Figure 3-2, right) $17.5 \pm 0.05$ compared to $1.0 \pm 0.2$ ). In contrast, Weri-1 Rb cells measured similar PDGFRA and PDGFRB mRNA expression (Figure 32, right); $4.5 \pm 0.5$ compared to $3.0 \pm 0.05)$. Because vitreous seeds are considered an aggressive phenotype, we focus the rest of the in vitro work on Y79 Rb cells.

There are multiple ligands within the PDGF family; there are four known homodimers (PDGF-AA, -BB, -CC, and -DD) and one heterodimer (PDGF-AB). Classically, PDGF-AA and -CC were known to bind and activate PDGFR $\alpha$; PDGF-BB 


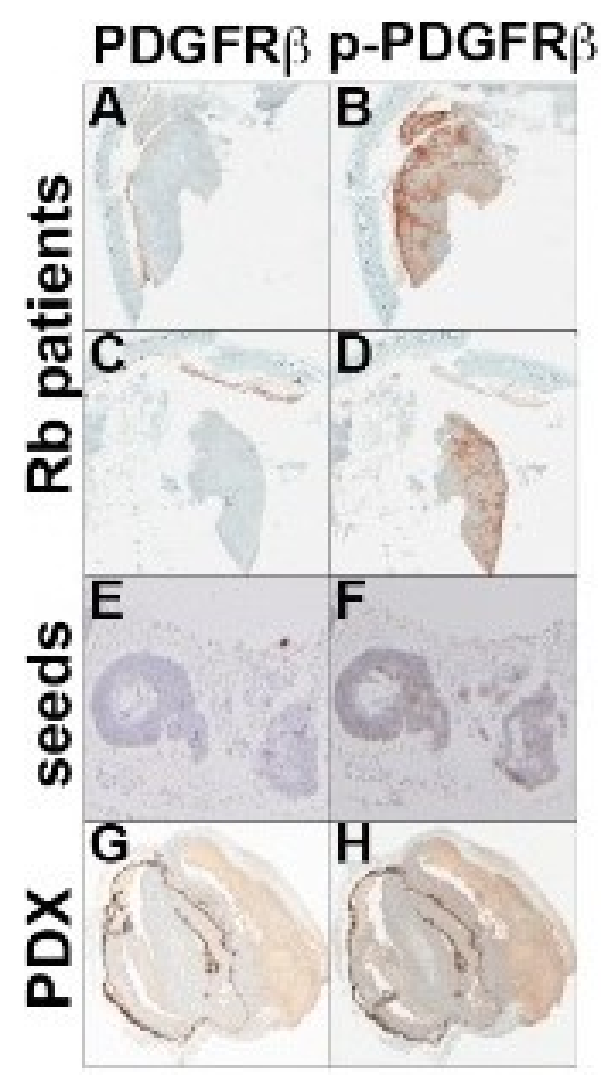

Figure 3-1. Immunohistochemistry of the active and non-active forms of PDGFR $\beta$ in $\mathbf{R b}$.

(A-D) Representative images of immunohistochemical staining for expression of PDGFR $\beta$ and phosphorylated PDGFR $\beta$ (p-PDGFR $\beta$ ) from enucleated eyes of $R b$ patients. (E-F) Representative image of immunohistochemical results of PDGFR $\beta$ in vitreous seeds. (G-H) Images from patient-derived orthotopic xenograft. Reprinted with permission. Goldsmith ZK, Coppess W, Irvine AS, et al. Targeting the Platelet-Derived Growth Factor-beta Stimulatory Circuitry to Control Retinoblastoma Seeds. Invest Ophthalmol Vis Sci. 2018;59(11):4486-4495. Copyright (2018), with permission through Creative Common CC-BY. 

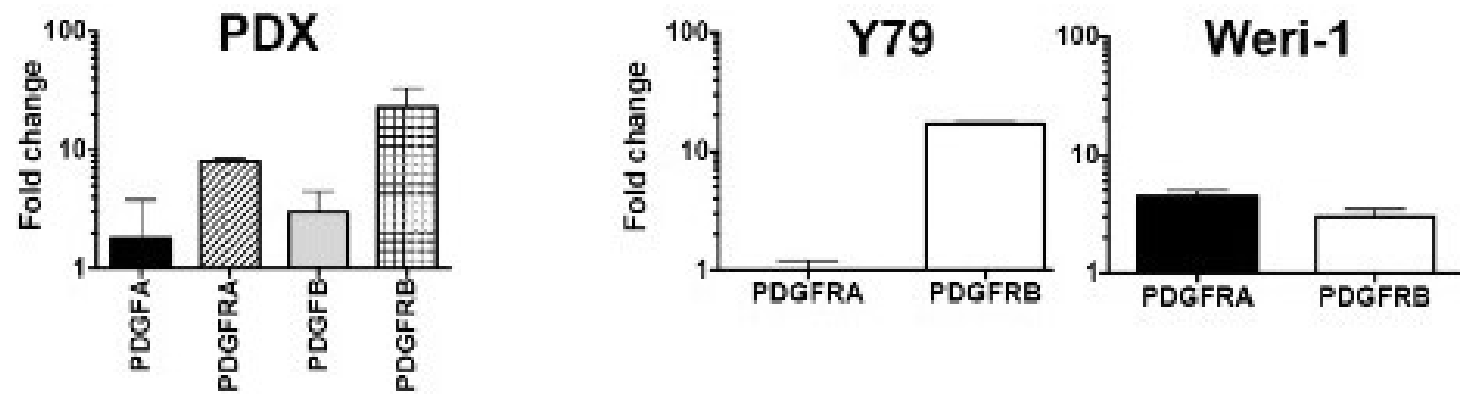

Figure 3-2. Expression of the key PDGF family members and the PDGFR $\beta$ in Rb.

qPCR analysis of mRNA isolated from both (left) PDX and (right) both Rb cell lines Y79 and Weri-Rb-1 for key members of PDGF signaling pathway. $\mathrm{N}=9$ in PDX mRNA analyses with 4 replicates per sample; $\mathrm{N}=4$ for $\mathrm{Y} 79$ and Weri-1 mRNA analyses with 4 replicates per sample; Reprinted with permission. Goldsmith ZK, Coppess W, Irvine AS, et al. Targeting the Platelet-Derived Growth Factor-beta Stimulatory Circuitry to Control Retinoblastoma Seeds. Invest Ophthalmol Vis Sci. 2018;59(11):4486-4495. Copyright (2018), with permission through Creative Common CC-BY. 
and -DD activated PDGFR $\beta$, and PDGF-AB was capable inducing activation of both homodimeric receptors (PDGFR $\alpha$ and PDGFR $\beta$ ) as well as the heterodimeric receptor PDGFR $\alpha \beta$ ). As we had previously demonstrated activation of PDGFR $\beta$, we hypothesized this activation would be attributable to an abundance of either PDGF-AB or PDGF-BB within the vitreous. To investigate the abundance of these ligands within the vitreous, we collected samples of vitreous from eyes of $\mathrm{Rb}$ patients that were primarily enucleated as a part of an ongoing clinical trial (NCT01783535) and compared the protein levels to those of healthy controls (purchased, BioreclamationIVT, Baltimore, MD) by Enzyme-linked Immunosorbent Assay (ELISA). We discovered PDGF-BB (Figure 3-3, left) to be more abundant in the Rb samples compared to those of healthy controls ( $\mathrm{Rb}$ samples: $360.22 \pm 80.261 \mathrm{pg} / \mathrm{mL}$, healthy controls: below the level of detection; $\left.{ }^{* *} p<0.005\right)$. Similarly, the levels of PDGF-BB were higher in the Rb samples compared to healthy controls $(1728.73 \pm 297.73$ compared to $332.70 \pm 76.220 \mathrm{pg} / \mathrm{mL}$; $* * * p<0.0005)$. The levels of PDGF-BB in the Rb samples were 5.19-fold higher than the healthy controls (Figure 3-3, right). Together, this work illustrates high levels of the pPDGFR $\beta$ that may be attributable to the abundance of its primary stimulatory ligand, PDGF-BB.

\section{Pharmacological disruption of PDGFR $\beta$ signaling reduced Rb proliferation, invasion, and increased cell death}

The PDGFR $\beta$ signaling pathway has been implicated in a number of cancers and ophthalmic diseases. As Rb is considered an orphan disease and has a relatively low incidence, studies aimed at identifying novel targets of therapeutic value are limited; therefore, the ideal candidate to disrupt PDGFR $\beta$ signaling would be a therapy that is currently utilized within the pediatric population and is already FDA-approved for treatment of tumors and, ideally, pediatric malignancies ${ }^{236,237}$. Therefore, to conduct our studies, we chose to utilize imatinib mesylate (IM) (marketed by Novartis as Gleevec ${ }^{\circledR}$ ) to disrupt the PDGFR $\beta$ pathway and investigate the role(s) it may play in Rb. IM was an ideal candidate as it is already clinically approved to treat both chronic myeloid leukemia (CML) and acute lymphocytic leukemia (ALL) within the pediatric population, as well as gastrointestinal stromal tumors (GISTs). We generated a dose-response curve and determined the most efficacious dose at controlling cellular proliferation was $10 \mu \mathrm{M}$. The disruption of the PDGFR $\beta$ signaling pathway was confirmed by Western blotting shown (Figure 3-4, left). We measured increased levels of PDGFR $\beta$ activity when recombinant human PDGF (rhPDGF) is added to Y79 cells $\left(0.415 \pm 0.05 ;{ }^{*} p<0.05\right)$. In contrast, there is a striking reduction of the receptor signaling activity when cells were treated with IM $(0.223 \pm 0.05 ; * * p<0.005)$ or rhPDGF + IM $(0.151 \pm 0.05 ; * * p<0.005)$, which mimics the physiology of the disease and treatment in the vitreous microenvironment, compared to rhPDGF. Next, we quantified the percentage of Y79 PDGFR $\beta^{+}$cells by flow cytometry analysis and found a significant reduction of Y79 cells expressing the PDGFR $\beta$ after IM treatment compared to untreated and rhPDGF-treated cells (rhPDGF: $86.10 \% \pm 4.0$, IM: $58.36 \% \pm 2.8$, rhPDGF + IM: $61.33 \% \pm 6.7 ;{ }^{*} p<0.005$ ), as shown in Figure 3-4, right. We measured cell viability by the MTS cellular proliferation assay. The results showed a 

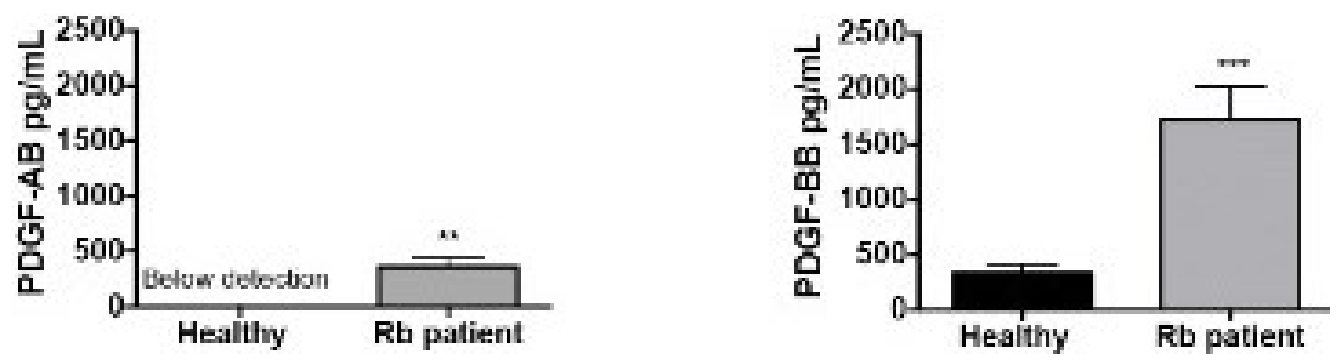

Figure 3-3. Abundance of PDGF ligands in the vitreous of Rb patients.

PDGF-AB and PDGF-BB ELISAs were used to quantify levels of protein within the vitreous microenvironment. Levels of PDGF-AB (left) and PDGF-BB (right) in vitreous samples from healthy controls vitreous compared to those of $\mathrm{Rb}$ patients. $\mathrm{N}=6$ in ELISA analyses done in triplicates. All results represent mean $\pm \mathrm{SEM} ; * * p<0.005 ; * * * p<0.0005$. Reprinted with permission. Goldsmith ZK, Coppess W, Irvine AS, et al. Targeting the Platelet-Derived Growth Factor-beta Stimulatory Circuitry to Control Retinoblastoma Seeds. Invest Ophthalmol Vis Sci. 2018;59(11):4486-4495. Copyright (2018), with permission through Creative Common CC-BY.
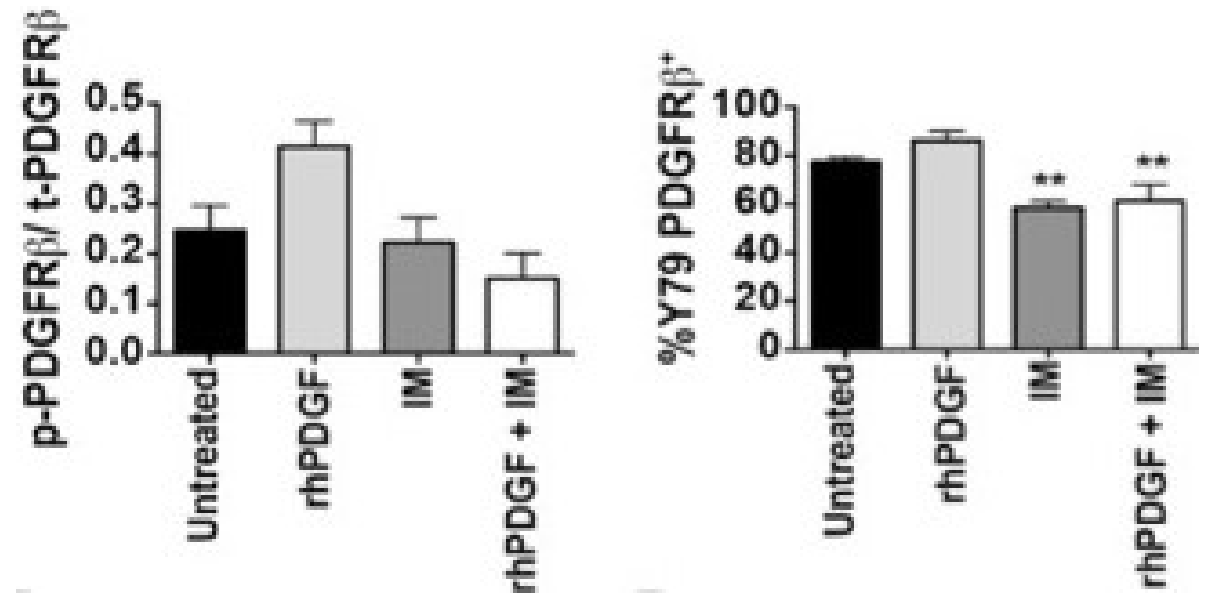

Figure 3-4. Imatinib mesylate disrupts PDGF-PDGFR $\beta$ signaling.

Y79 cells were cultured in the presence or absence of either PDGF-BB $(10 \mathrm{ng} / \mathrm{mL})$ or the PDGFR $\beta$ inhibitor imatinib mesylate $(\mathrm{IM}, 10 \mu \mathrm{M})$. Western blot analyses of the activity of the PDGFR $\beta$ by measurement of the ratio of phosphorylated-PDGFR $\beta$ to total PDGFR $\beta$ (left) Flow cytometry analyses showing the percentage of Y79 cells that express PDGFR $\beta$ (right). $\mathrm{N}=3$ and 3 replicates per sample. All results represent mean \pm SEM; ${ }^{*} p<0.005$. Reprinted with permission. Goldsmith ZK, Coppess W, Irvine AS, et al. Targeting the Platelet-Derived Growth Factor-beta Stimulatory Circuitry to Control Retinoblastoma Seeds. Invest Ophthalmol Vis Sci. 2018;59(11):4486-4495. Copyright (2018), with permission through Creative Common CC-BY. 
significant increase in proliferation over 120h in the presence of rhPDGF illustrating a potential mitogenic role. This mitogenic effect was inhibited by IM and rhPDGF + IM treatment (Figure 3-5, left $*^{*} p<0.05,{ }^{* *} p<0.005, * * * p<0.0005$ ). We then investigated if inhibition of PDGFR $\beta$ could increase cell death (Figure 3-5, right) by flow cytometry analysis of apoptotic (Annexin $\mathrm{V}^{+} \mathrm{PI}^{+}$) cells. At the time of setting up the cell cultures we measured cell death of Y79 cells to obtain the baseline levels. We measured cell death by using Annexin V and PI and found about 3\% cell death. To confirm these results, we used Zombie Aqua $^{\mathrm{TM}}$, which is a sensitive amine-reactive dye that is non-permeant to live cells and found $5.8 \%$ of cell death. There was a significant increase in cell death in IM $(19.51 \% \pm 5.3 ; * p<0.05)$ and $\mathrm{rhPDGF}+\mathrm{IM}(27.77 \% \pm 2.7 ; * * * p<0.0005)$ compared to untreated $(11.55 \% \pm 0.6)$ and rhPDGF $(13.16 \% \pm 0.8)$. These results illustrated the role of the PDGFR $\beta$ signaling in $\mathrm{Rb}$ cell growth and death.

As Y79 cells represent the aggressive and metastatic model of disease, we measured the invasive potential of these cells to test if the PDGF-PDGFR $\beta$ signaling pathway controls invasion by culturing them in well inserts coated with basement membrane ${ }^{170}$. We measured the ability of cells to invade basement membrane via quantitation of cells per field (Figure 3-6). This data demonstrated IM (139.66 \pm 10.3 ; $\left.{ }^{*} p<0.05\right)$ and rhPDGF + IM $\left(163.33 \pm 6.0 ;{ }^{*} p<0.05\right)$ treated cells showed less invasion

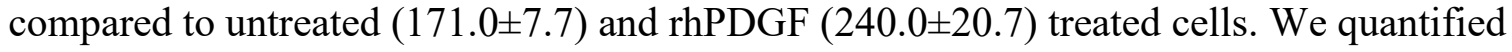
the cells that crossed the membrane by removal of the non-invasive cells followed by labeling of the invasive cells. Representative images of cells in each condition are shown (Figure 3-6, right).

\section{Disruption of PDGFR $\beta$ targets the MDM2 and NFkB signaling cascades}

The primary goal of this research is to understand more about how interactions with the TME may promote $\mathrm{Rb}$ cell survival. One such mechanism by which cells are capable of surviving is through inhibition of apoptosis by key cell signaling molecules, such as MDM2 and NFkB signaling. These two molecules are linked to anti-apoptotic mechanisms that have yet to be studied within the context of the PDGFR $\beta$ pathway in $\mathrm{Rb}$. We hypothesized that disruption of the PDGFR $\beta$ pathway by IM, could result in decreases in activity of both MDM2 and NFKB that could account for the increases in apoptotic cells we demonstrated earlier. Hence, we investigated the in-depth mechanisms by which PDGFR $\beta$ controls Rb cell survival and death. Quantitative PCR analysis demonstrated downregulation of MDM2 mRNA after disruption of the PDGFR $\beta$ pathway (IM: $1.565 \pm 0.41$, rhPDGF + IM: $1.905 \pm 0.48)$ compared to rhPDGF-treated (4.306 \pm 0.44$)$ cells (Figure 3-7A). A similar effect was measured when MDM4 mRNA, another regulator of tumor suppression, was tested (Figure 3-7B). We then examined MDM2 signaling activity by Western blot analysis and discovered MDM2 signaling is impaired in IM $(0.5 \pm 0.2 ; * p<0.05)$ and rhPDGF + IM-treated $(0.4 \pm 0.001 ; * *<<0.005)$ cells compared to the levels of untreated (1.0 \pm 0.0$)$ and rhPDGF $(1.1 \pm 0.1)$ (Figure 3-7C). We further hypothesized this key signaling event could be at least partially regulated by the 

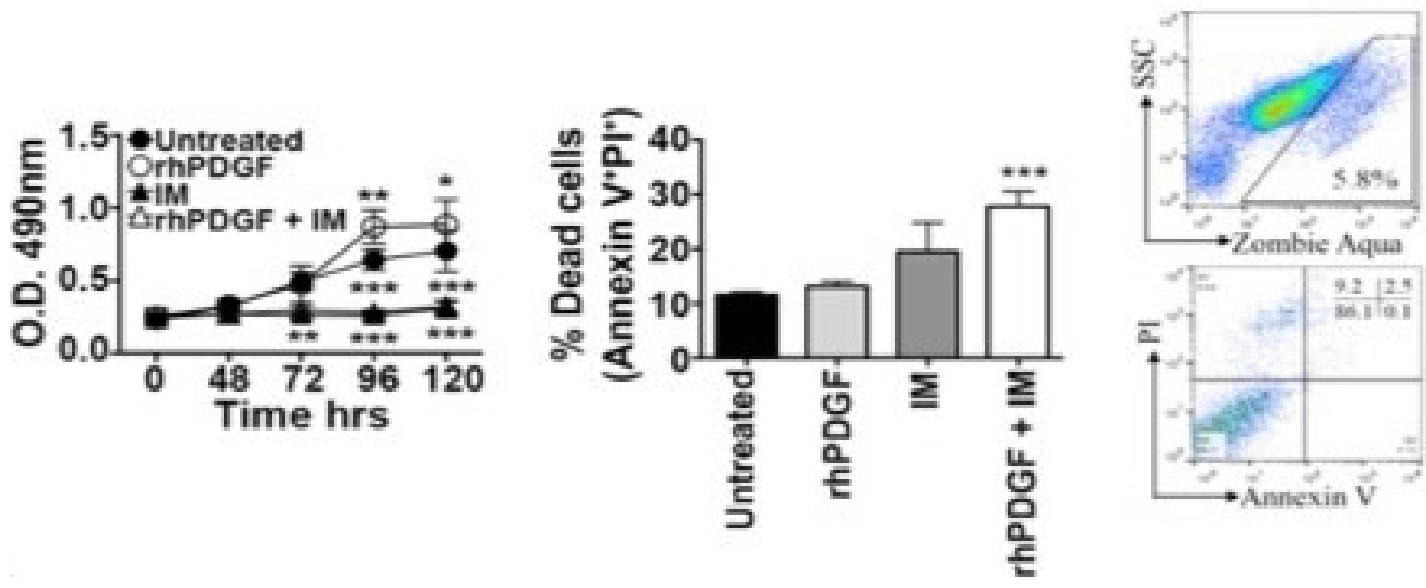

Figure 3-5. PDGF-PDGFR $\beta$ signaling induces Rb cell proliferation and regulates survival.

Y79 cells were cultured in the presence or absence of either PDGF-BB $(10 \mathrm{ng} / \mathrm{mL})$ or the PDGFR $\beta$ inhibitor imatinib mesylate (IM, $10 \mu \mathrm{M})$. Left, Assessment of Y79 cellular proliferation over time in the presence of either PDGF-BB or IM using the concentrations described above. Middle, The percentage cells undergoing apoptosis was measured by Annexin V and PI labeling using flow cytometry. Dead cells displayed an Annexin $\mathrm{V}^{+} \mathrm{PI}^{+}$ phenotype. Right, Evaluation of cell viability by Zombie Aqua labeling (top) or Annexin $\mathrm{V}^{+} \mathrm{PI}^{+}$(bottom) of $\mathrm{Y} 79 \mathrm{Rb}$ cells at $0 \mathrm{hr}$. $\mathrm{N}=3$ and 3 replicates per sample. All results represent mean $\pm \mathrm{SEM} ;{ }^{*} p<0.05,{ }^{* *} p<0.005,{ }^{* * *} p<0.0005$. Reprinted with permission. Goldsmith ZK, Coppess W, Irvine AS, et al. Targeting the Platelet-Derived Growth Factor-beta Stimulatory Circuitry to Control Retinoblastoma Seeds. Invest Ophthalmol Vis Sci. 2018;59(11):4486-4495. Copyright (2018), with permission through Creative Common CC-BY. 

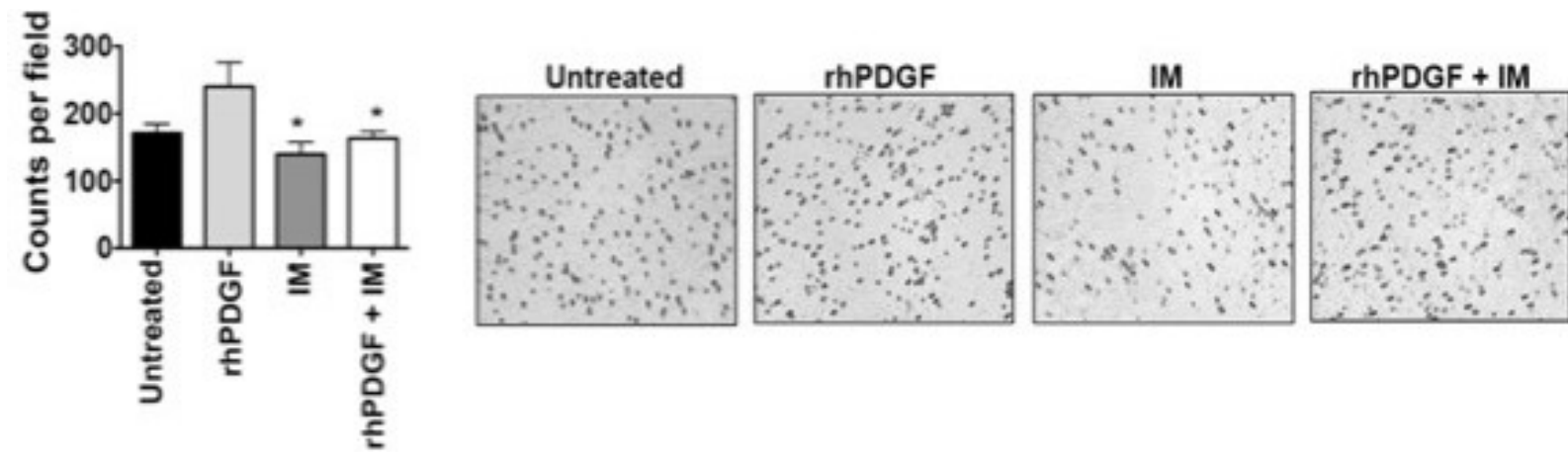

Figure 3-6. Reduction in invasive capacity of Y79 cells after IM treatment on basement membrane-coated inserts.

Y79 cells were cultured in the presence or absence of either PDGF-BB $(10 \mathrm{ng} / \mathrm{mL})$ or the PDGFR $\beta$ inhibitor imatinib mesylate (IM, $10 \mu \mathrm{M}$ ). (left) total counts per field, and (right) representative images of fields counted. $\mathrm{N}=3$ and 3 replicates per sample. All results represent mean $\pm \mathrm{SEM} ;{ }^{*} p<0.05$. Reprinted with permission. Goldsmith ZK, Coppess W, Irvine AS, et al. Targeting the Platelet-Derived Growth Factor-beta Stimulatory Circuitry to Control Retinoblastoma Seeds. Invest Ophthalmol Vis Sci. 2018;59(11):4486-4495. Copyright (2018), with permission through Creative Common CC-BY. 


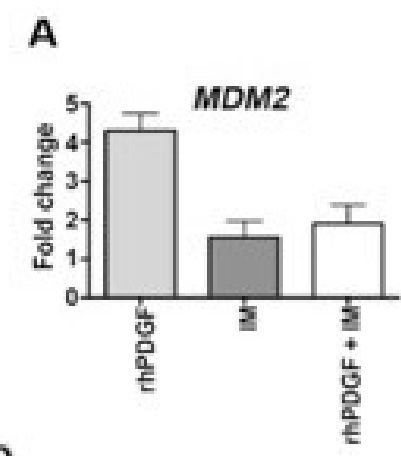

B

C
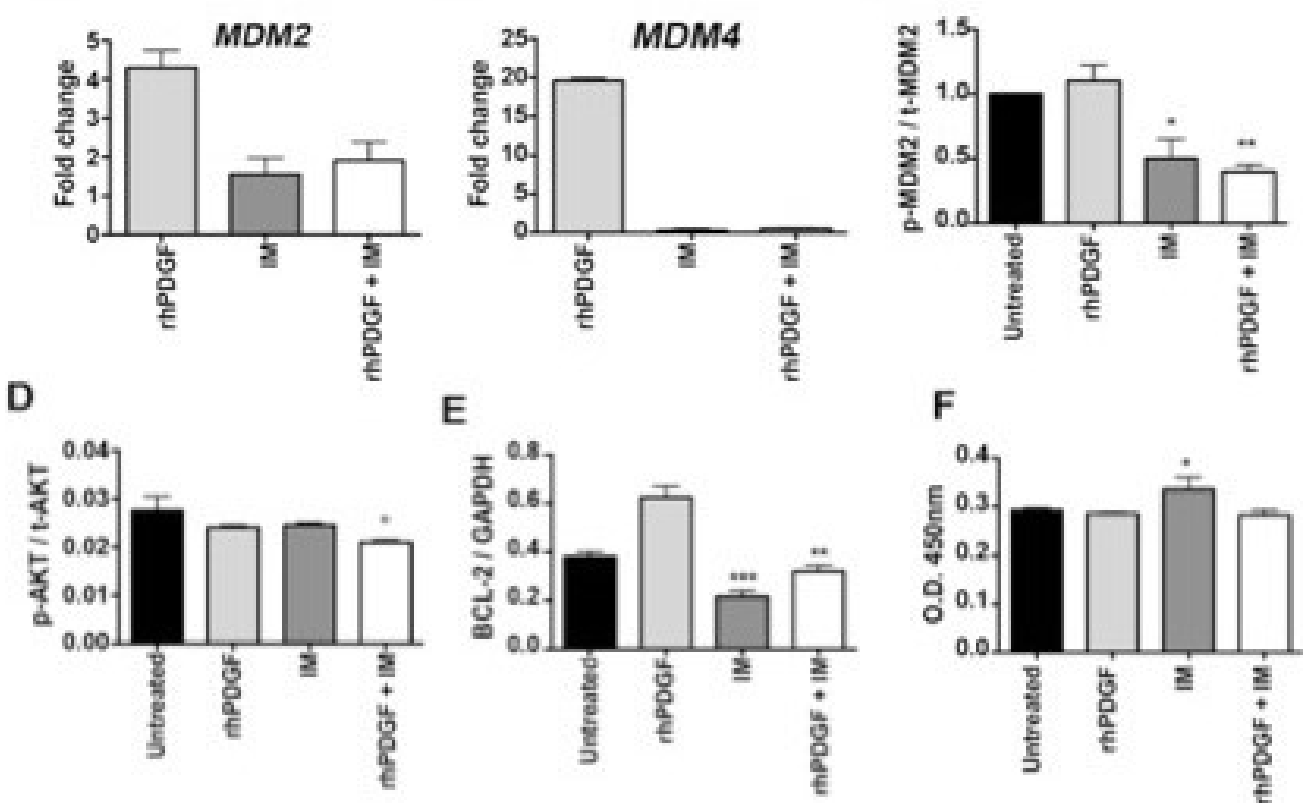

E

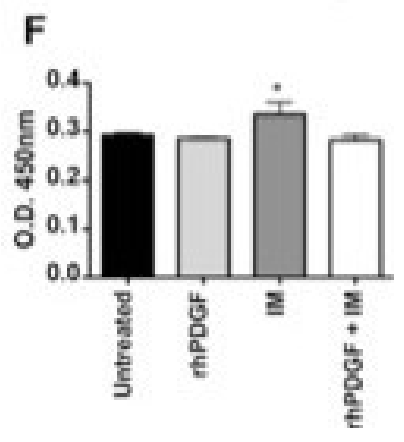

Figure 3-7. PDGF-PDGFR $\beta$ signaling regulates cell survival signaling.

Y79 cells were cultured in the presence or absence of either PDGF-BB $(10 \mathrm{ng} / \mathrm{mL})$ or the PDGFR $\beta$ inhibitor imatinib mesylate (IM, $10 \mu \mathrm{M}$ ). (A-B) qPCR analyses on mRNA levels of (A) MDM2 and (B) MDM4 relative to untreated Y79 cells under the cell culture conditions described above. (C-D) Western blot analyses (C) of MDM2 activity and (D) AKT activity after disruption of the PDGFR $\beta$ signaling cascade. (E-F) Evaluation of the anti-apoptotic mediator (E) BCL-2 by Western blot and the pro-apoptotic cleaved (active) capsase- 3 by ELISA (F). $\mathrm{N}=3$ and 3 replicates per sample. All results represent mean \pm SEM; ${ }^{*} p<0.05,{ }^{* *} p<0.005,{ }^{* * *} p<0.0005$. Reprinted with permission. Goldsmith ZK, Coppess W, Irvine AS, et al. Targeting the Platelet-Derived Growth Factor-beta Stimulatory Circuitry to Control Retinoblastoma Seeds. Invest Ophthalmol Vis Sci. 2018;59(11):4486-4495. Copyright (2018), with permission through Creative Common CC-BY. 
upstream activator of MDM2, AKT and, therefore, investigated if the reduction in cell proliferation is AKT-dependent. A small, albeit significant $\left({ }^{*} p<0.05\right)$, modulation of AKT signaling activity in rhPDGF + IM-treated cells (Figure 3-7D).

After examination of MDM2 and AKT, both involved in cell survival pathways, we investigated the mechanisms by which PDGFR $\beta$ controls cell death in $\mathrm{Rb}$. We postulated this disruption of AKT could also affect other downstream targets involved in apoptotic signaling, most notably the anti-apoptotic molecular BCL-2, and an executioner caspase, Caspase-3. We first assessed the levels of the anti-apoptotic BCL-2 protein after PDGFR $\beta$ disruption. Y79 cells treated with IM and rhPDGF + IM have a significant reduction in BCL-2 levels (Figure 3-7E). We also investigated the role of cleaved Caspase-3, the final effector in inducing programmed cell death. We measured the abundance of cleaved Caspase-3 by ELISA to investigate if the BCL-2 reduction was concomitant to an increase in cleaved Caspase-3 (Figure 3-7F). IM-treated cells showed a significant increase in cleaved Caspase-3 $\left(0.336 \pm 0.01 ;{ }^{*} p<0.05\right)$ compared to the rest of the cell culture conditions (untreated: $0.293 \pm 0.002$, rhPDGF: $0.286 \pm 0.007$ ).

Our next investigation aimed to evaluate if inhibition of PDGFR $\beta$ signaling could result in a potential compensatory effect in VEGF-VEGFR2 signaling, which is expressed in $\mathrm{Rb}^{170,238,239}$. Previous work from our lab (see Chapter 2 above; Figure 2-6A) demonstrated how targeting and inhibiting the MMPs, VEGF levels would increase or decrease in a cell-dependent manner and this could alter the TME to become more (or less) angiogenic, for example. Therefore, in a similar manner, we hypothesized inhibition of PDGFR $\beta$ would result in a similar decrease in VEGF levels that could decrease VEGF-VEGFR signaling. We confirmed the expression of VEGFA mRNA in Y79 cells and in the PDX model of Rb (Figure 3-8A-B). Protein analysis through VEGF ELISA revealed elevated levels of VEGF in the vitreous of $\mathrm{Rb}$ patients compared to those of health vitreous (Figure 3-8C). Additional qPCR analyses on Y79 cells revealed no difference in VEGFA and VEGFR2 mRNA expression after disruption of the PDGFR $\beta$ signaling (Figure 3-8D-E). Despite no changes at the transcriptional level there was a reduction in VEGFR2 signaling after treatment with IM (Figure 3-8F) (IM: 0.93 \pm 0.06 , rhPDGF + IM: $0.77 \pm 0.04 ; * p<0.05$ ) compared to the controls (untreated: $1.00 \pm 0.04$, rhPDGF: $1.00 \pm 0.007)$. These results demonstrate targeting PDGF-PDGFR $\beta$ signaling does not result in a compensatory mechanism through VEGF-VEGFR2.

The disruption of PDGFR $\beta$ signaling via IM showed this pathway plays a role in $\mathrm{Rb}$ tumor cell survival. We hypothesized the suppression of Y79 survival may occur via a crosstalk between MDM2 and NFKB. To address this, we utilized imaging flow cytometry to measure the nuclear translocation of the NFkB p65 subunit. Quantitative analysis showed a distinct reduction in the percentage of cells showing p65 nuclear translocation (Figure 3-9A) after IM treatment (IM: 17.8\% 0.7 , rhPDGF + IM: $22.8 \% \pm 0.8 ; * * * p<0.0005)$ compared to the untreated $(33.0 \% \pm 0.0)$ and rhPDGF $(45.0 \% \pm 0.0)$ controls. Along with a reduction on the percentage of cells showing p65 nuclear translocation, we measured an overall reduction in the p65 protein by measurement of the mean fluorescent intensity (Figure 3-9B) in the presence of IM (IM: 
A

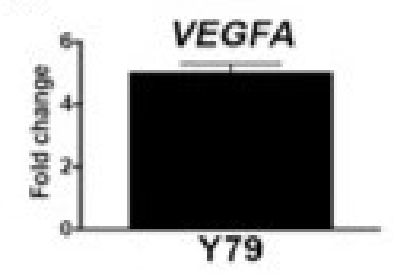

D

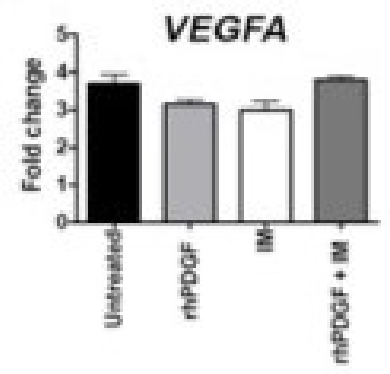

B

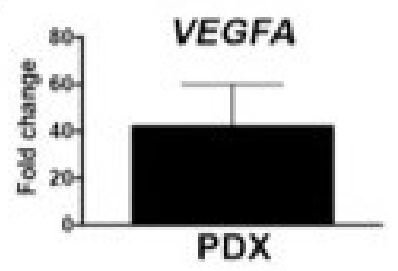

$\mathbf{E}$

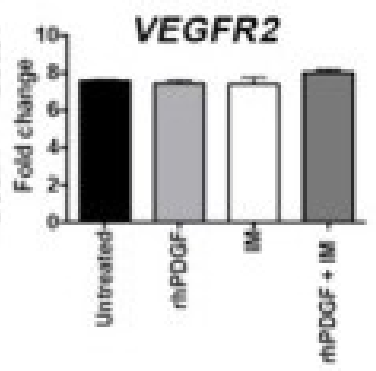

C

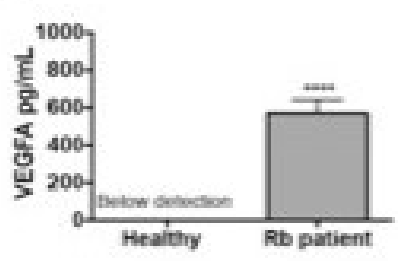

F

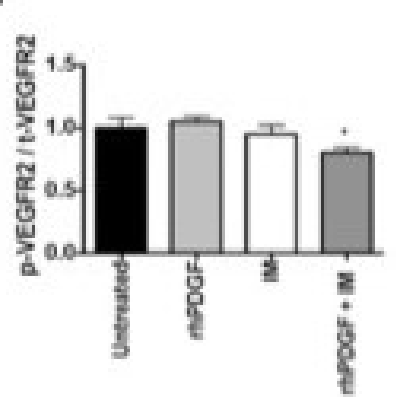

Figure 3-8. Lack of VEGF-VEGFR2 compensatory mechanism when PDGFPDGFR $\beta$ signaling is disrupted.

(A-B) VEGFA mRNA levels were measured by qPCR analysis in both Y79 (A) cells and PDX (B). (C) Measurement of VEGFA levels in a cohort of vitreous samples from healthy controls compared to vitreous of Rb patients. Y79 cells were cultured in the presence or absence of either PDGF-BB $(10 \mathrm{ng} / \mathrm{mL})$ or the PDGFR $\beta$ inhibitor imatinib mesylate $(\mathrm{IM}, 10 \mu \mathrm{M})$. (D-E) Both $V E G F A$ (D) and VEGFR2 (E) mRNA levels were measured across respective treatments as well as (F) Western blot analyses of VEGFR2. Experiments from (A,C-F), done with $\mathrm{N}=3$ and each sample done in triplicates. Experiment from (B) $\mathrm{N}=9$ and each sample done in replicates of $4 . \mathrm{N}=6$ in ELISA analyses done in triplicates. All results represent mean \pm SEM; ${ }^{*} p<0.05$. Reprinted with permission. Goldsmith ZK, Coppess W, Irvine AS, et al. Targeting the Platelet-Derived Growth Factor-beta Stimulatory Circuitry to Control Retinoblastoma Seeds. Invest Ophthalmol Vis Sci. 2018;59(11):4486-4495. Copyright (2018), with permission through Creative Common CC-BY. 

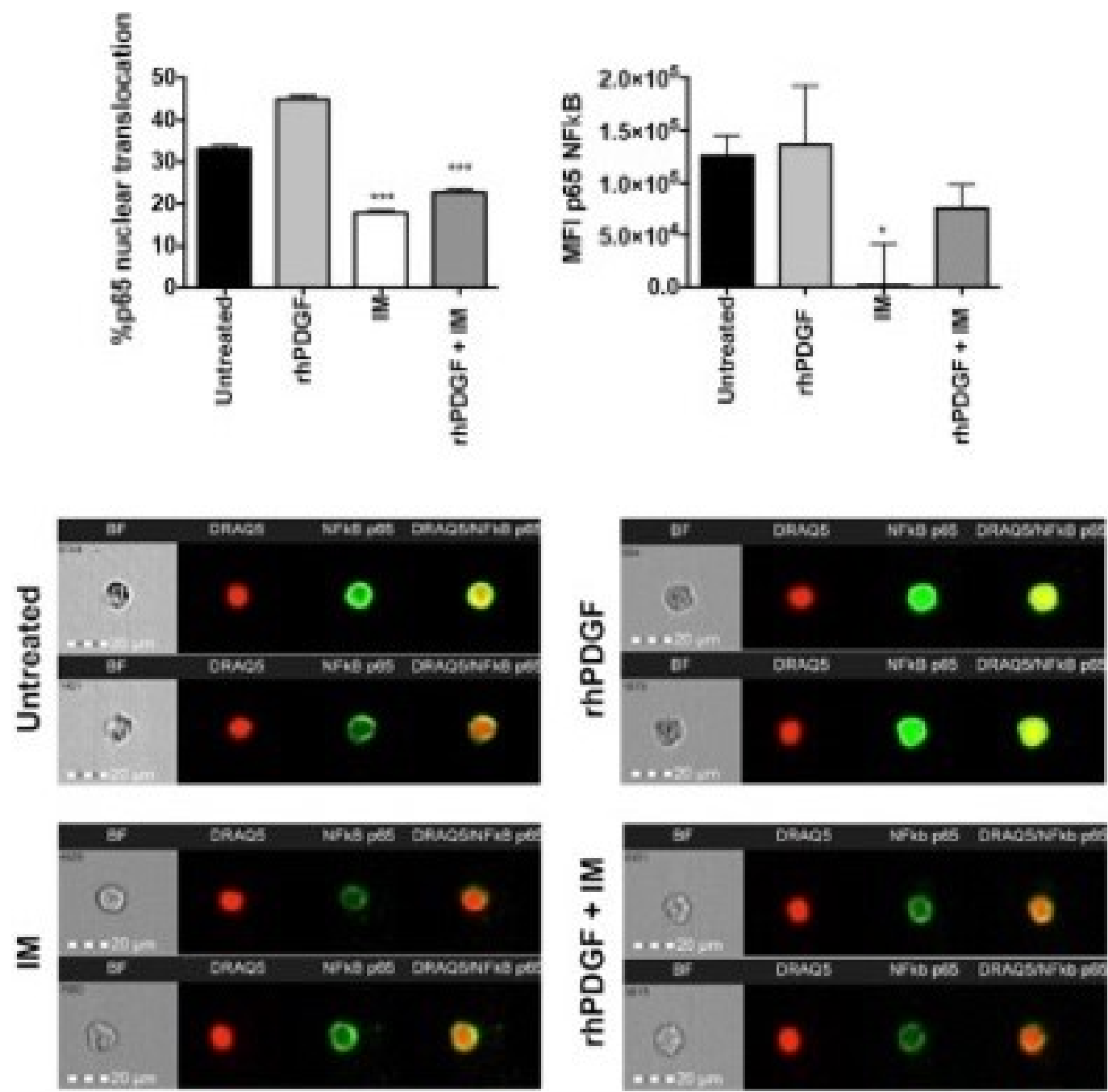

Figure 3-9. Disrupting PDGF-PDGFR $\beta$ signaling decreases NFkB nuclear translocation.

Y79 cells were cultured in the presence or absence of either PDGF-BB $(10 \mathrm{ng} / \mathrm{mL})$ or the PDGFR $\beta$ inhibitor imatinib mesylate (IM, $10 \mu \mathrm{M}$ ). (A-C) Assessment of the NFKB signaling, (top left) the percentage of treated Y79 cells with nuclear localization of the p65 subunit and (top right) the expression (or mean fluorescent intensity) of the p65 subunit using an Amnis FlowSight ${ }^{\circledR}$ Imaging Cytometer. Representative images of Y79 cells (bottom) labeled and analyzed for nuclear (labeled with DRAQ5 ${ }^{\mathrm{TM}}$ ) translocation of the $\mathrm{p} 65$ subunit of $\mathrm{NF \kappa B}$ (AlexaFluor 488 conjugated). Experiments from A-C tested 10,000 cells. All results represent mean $\pm \mathrm{SEM} ; *<0.05,{ }^{* * *} p<0.0005$. Reprinted with permission. Goldsmith ZK, Coppess W, Irvine AS, et al. Targeting the Platelet-Derived Growth Factor-beta Stimulatory Circuitry to Control Retinoblastoma Seeds. Invest Ophthalmol Vis Sci. 2018;59(11):4486-4495. Copyright (2018), with permission through Creative Common CC-BY. 
$1.627 \times 10^{3} \pm 3.0 \times 10^{4}$, rhPDGF + IM: $\left.7.5 \times 10^{4} \pm 2.3 \times 10^{4} ;{ }^{*} p<0.05\right)$ compared to the controls (untreated: $1.25 \times 10^{5} \pm 2.0 \times 10^{4}$, rhPDGF: $1.35 \times 10^{5} \pm 5.6 \times 10^{4}$ ). Representative images of the p65 nuclear localization analyses are shown in Figure 3-9C. Co-localization of p65 (green) to the nucleus (red) is depicted in yellow. Collectively, our work supports our hypothesis that the PDGFR $\beta$ promotes Rb cell survival and death via modulation of MDM2 and nuclear translocation of the pro-survival NFKB molecule.

\section{Reduction of Rb spheroids by IM in a 3D cell culture model of Retinoblastoma vitreous seeds}

There have been no in vitro models for $\mathrm{Rb}$ vitreous seeds. As a result of a lack of proper in vitro models, studies on vitreous seeds are quite limited. To investigate vitreous seeds, studies would need to be done either using ex vivo human tissue or in vivo using the rabbit xenograft model ${ }^{92}$. To understand more about their in vivo development and behavior, an in vitro model could prove quite valuable. Furthermore, an in vitro model of vitreous seeds would provide a novel tool to develop drug screens while preclinically testing efficacy of potential future therapeutics. We adapted our in vitro 3D cell culture system to recreate $\mathrm{Rb}$ seeds in a vitreous-like microenvironment for in-depth study. Schematics of the procedure are shown in Figure 3-10A. These "seeds" were generated by magnetic levitation and the resulting spheroids were visible to the naked eye (Figure 3-10B). Multiple aggregates or clusters of cells are found within the cell cultures. Using the 3D system, we are able to generate a spheroid that forms one large mass by addition of magnetic nanoshuttles to our cell culture that is further magnetized. The viability of Y79 cells in a standard cell culture system was compared to that of the 3D system. Quantitative analysis of live cells by trypan blue exclusion (Figure 3-10C) revealed no difference in the viability of cells after overnight culture. We compared images of our magnetically-generated $\mathrm{Rb}$ spheroids to ex vivo $\mathrm{Rb}$ seeds found similar morphology (Figure 3-10D).

We then measured the area of the spheroids using Image $(\mathrm{NIH})$ over time after treatment with IM. We discovered a reduction in the area of $\mathrm{Rb}$ spheroids $48 \mathrm{hrs}$ after rhPDGF + IM treatment $\left({ }^{*} p<0.05\right)$ compared to the control groups (Figure 3-11A). Y79 cells were labeled with carboxyfluorescein succinimidyl ester (CFSE) to trace and visualize the cells fluorescently. We observed the center of the spheroid to have fewer cells than the rest of the spheroid (Figure 3-11B, shown at 48h) when $\mathrm{CFSE}^{+} \mathrm{Rb}$ spheroids were cultured over time. The size of the translucent center is larger in those spheroids treated with IM (both IM and rhPDGF + IM) compared to the control groups, suggesting death. We confirmed our flow cytometry findings (Figure 3-4) of a qualitative reduction in the expression of PDGFR $\beta$ and VEGFR2 in IM treated-cells (Figure 3-11C) by using immunofluorescence microscopy in the 3D system. Through the use of a 3D cell culture model we generated $\mathrm{Rb}$ spheroids that showed a reduction in the spheroid area after disruption of the PDGFR $\beta$ signaling as well as a lack of VEGFR2 activity. 


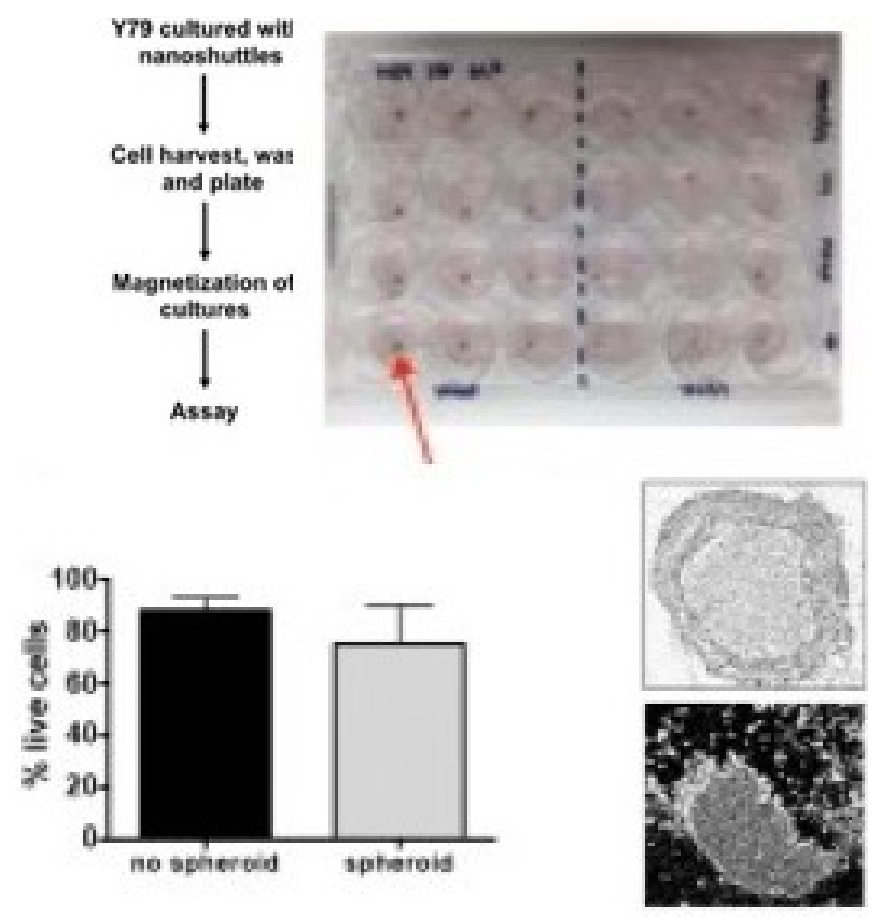

Figure 3-10. Three-dimensional magnetic tumor spheroids as an in vitro model for vitreous seeds.

Using commercially available magnetic nanoshuttles, (top) Y79 cells were magnetized to form three-dimensional tumor spheroids (see: red arrow). (bottom left) Trypan blue exclusion was used to measure cell viability in samples with spheroid formation versus non-spheroid. (Bottom right, top) Comparison of an ex vivo vitreous seed to (Bottom right, bottom) a representative three-dimensional tumor spheroid. All results in (C) represent mean \pm SEM. Reprinted with permission. Goldsmith ZK, Coppess W, Irvine AS, et al. Targeting the Platelet-Derived Growth Factor-beta Stimulatory Circuitry to Control Retinoblastoma Seeds. Invest Ophthalmol Vis Sci. 2018;59(11):4486-4495. Copyright (2018), with permission through Creative Common CC-BY. 

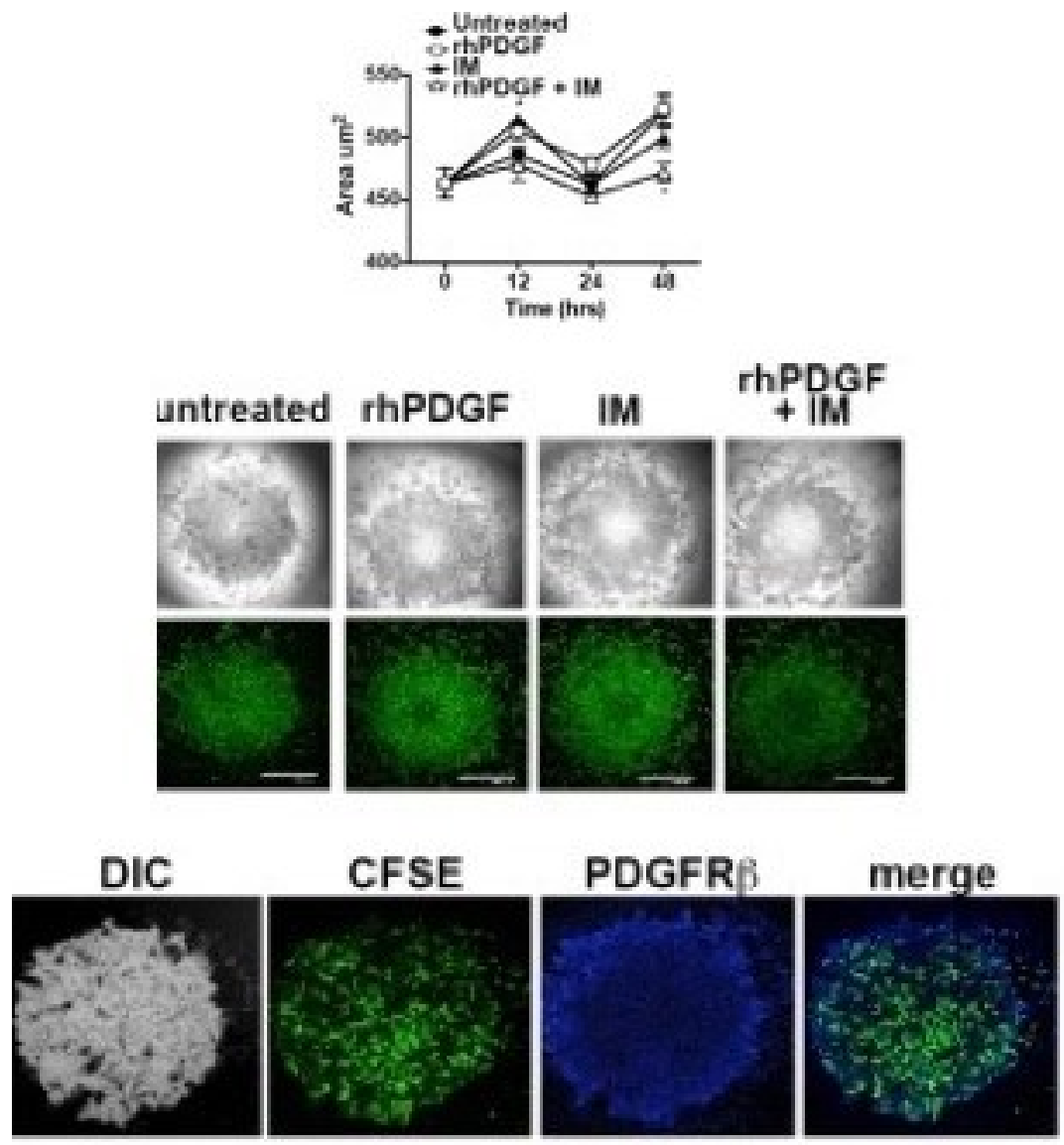

Figure 3-11. Quantitative and qualitative assessment of first in vitro model of vitreous seeds.

Using commercially available magnetic nanoshuttles, (top) Measurements of magnetized $\mathrm{Rb}$ spheroid area over time. CFSE-labeled Rb spheroids were assessed (middle) morphologically and for PDGFR $\beta$ (bottom) expression $48 \mathrm{hrs}$ after culture. All results in top represent mean $\pm \mathrm{SEM} ;{ }^{*} p<0.05$. In imaging experiments, a minimum of 3 wells of spheroids were generated per culture condition. Reprinted with permission. Goldsmith ZK, Coppess W, Irvine AS, et al. Targeting the Platelet-Derived Growth Factor-beta Stimulatory Circuitry to Control Retinoblastoma Seeds. Invest Ophthalmol Vis Sci. 2018;59(11):4486-4495. Copyright (2018), with permission through Creative Common CC-B 


\section{Reduction in cell viability, MDM2 signaling and $\mathrm{p} 65$ nuclear translocation are PDGFR $\beta$-specific}

To confirm if the reduction of cell viability was PDGFR $\beta$-specific or an off-target effect of the IM drug we knocked down PDGFRB by siRNA technology. We confirmed $P D G F R B$ mRNA knockdown after $P D G F R B$ siRNA transfection $(0.339 \pm 0.060)$ compared to samples transduced with random nucleotides (Scramble, $0.8 \pm 0.002$ ) and untreated (1.0 \pm 0.00$)$ controls (Figure 3-12A). We then evaluated the effect of PDGFRB gene knockdown on $\mathrm{Rb}$ cell survival and death. To address this, we labeled cells with Annexin V and PI labeling as before (Figure 3-5B) and analyzed by flow cytometry. The analysis showed a reduction in the percentage of live (Annexin $V^{\text {neg }} \mathrm{PI}^{\text {neg }}$ ) cells in $P D G F R B$ siRNA transfected cells compared to the Scramble and untreated controls (Figure 3-12B). The percentage of early apoptotic cells with a phenotype of Annexin $\mathrm{V}^{+} \mathrm{PI}^{\text {neg }}$ increased in PDGFRB siRNA transfected samples compared to untreated and Scramble-treated cells (Figure 3-12C). Similarly, the percentage of late apoptotic cells, defined as Annexin $\mathrm{V}^{+} \mathrm{PI}^{+}$, increased in PDGFRB siRNA transfected cells compared to untreated and Scramble-transfected cells (Figure 3-12D). The percentage of Annexin $\mathrm{V}^{+} \mathrm{PI}^{+}$cells was higher in Scramble-transfected cells compared to untreated cells. However, the magnitude of the increase in the percentage of late apoptotic cells in PDGFRB siRNA-transfected cells is larger in the Scramble-treated group. As part of our investigation on the role of PDGFR $\beta$ in Rb cell survival and death we measured MDM2 signaling in the siRNA transfections. Western blot demonstrated a reduction, not significant $(p=0.06)$, in MDM2 signaling in PDGFRB siRNA-transfected cells compared to the control group (Figure 3-12E).

Activation of NFKB may occur from multiple upstream cell-surface receptors. As some of these cell-service receptors are tyrosine kinases and IM is a TKI, we wished to confirm that disruption of p65 nuclear translocation was PDGFR $\beta$-specific and not the result of a non-specific effect of IM. We demonstrated a reduction in the p65 subunit of $\mathrm{NF \kappa B}$ and in the nuclear translocation after pharmacological disruption of PDGFR $\beta$ with IM (Figure 3-9). We hypothesized that, just as interference of PDGFRB by siRNA resulted in increases in percentage of apoptotic cells, we would also detect reductions in nuclear translocation of p65. To confirm this was a PDGFR $\beta$-dependent effect, we compared these parameters in PDGFRB siRNA-transfected cells to Scramble and untreated controls (Figure 3-12F). The results revealed a marked reduction of the percentage of cells showing p65 nuclear translocation in the PDGFRB siRNA-transfected $(9.10 \% \pm 0.45, * * * p<0.0005)$ cells (Figure 3-12E, untreated: $45.04 \% \pm 2.25$, Scramble: $38.33 \% \pm 2.9$ ) in addition to a reduction in the amount of p65 protein Figure 3-12G, $1.17 \times 10^{5} \pm 5.8 \times 10^{3}$ ) compared to the control groups (untreated: $1.82 \times 10^{5} \pm 8.1 \times 10^{3}$, Scramble: $2.03 \times 10^{5} \pm 1.1 \times 10^{3}$ ). Co-localization of p65 (green) to the nucleus (red) is shown in yellow in Figure 3-12H. Our PDGFRB siRNA transfection findings confirmed our results using the pharmacological inhibitor of PDGFR $\beta$ IM and support our hypothesis that the PDGFR $\beta$ plays an essential role in Rb cell survival and death, MDM2 signaling and $\mathrm{NF \kappa B}$. 
A

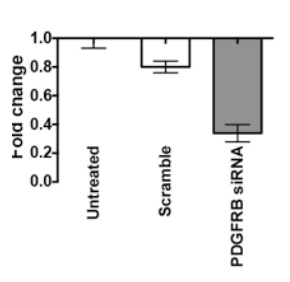

F

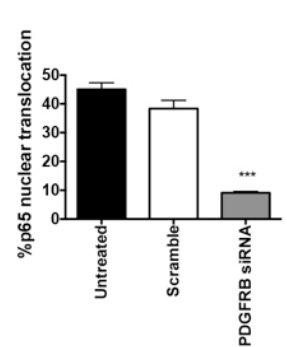

B

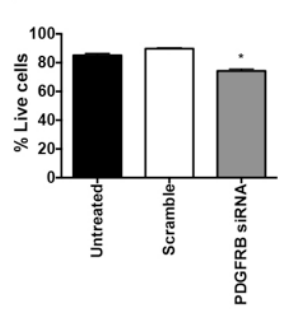

G $c$

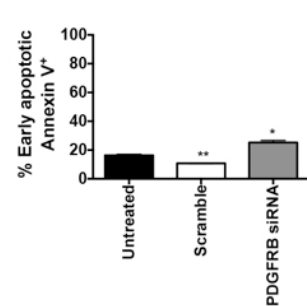

H
D

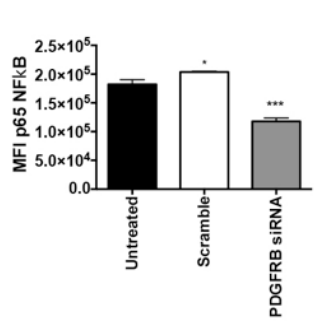

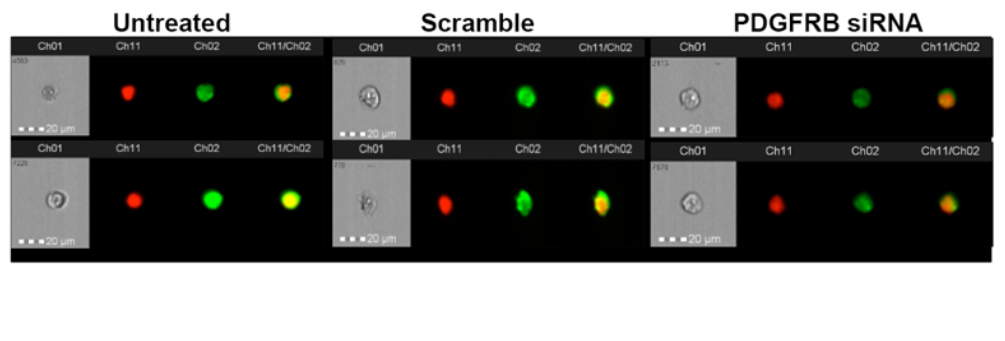

E

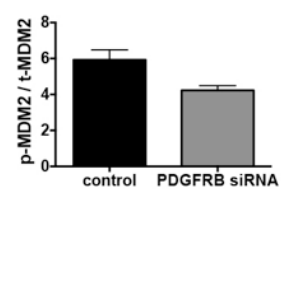

Figure 3-12. Confirmation of PDGFR $\beta$-specific effects in Rb cells by PDGFRB siRNA.

PDGFRB gene expression in Y79 was targeted using commercially available siRNA. (A) qPCR analyses confirmed effective knockdown of PDGFRB mRNA in siRNA transfected samples compared to non-specific oligonucleotides and untreated cells. The percentages of viable and apoptotic cells were determined by flow cytometry analysis using (B) the Annexin $\mathrm{V}^{\text {neg}} \mathrm{PI}{ }^{\text {neg }}$ phenotype for live cells, (C) the percentage of early apoptotic $\left(\right.$ Annexin $\mathrm{V}^{+}$) and (D) late apoptotic $\left(\right.$Annexin $\mathrm{V}^{+} \mathrm{PI}^{+}$) cells. (E). Western blot analyses were done to measure activity levels of MDM2 after transfection of PDGFRB siRNA. (F-H) Using the Amnis FlowSight ${ }^{\circledR}$ Imaging Cytometer, untreated, scramble, or PDGFRB siRNA- transfected Y79 cells were labeled and analyzed for $(\mathbf{F})$ the percentage of p65 nuclear translocation and (G) the expression (mean fluorescent intensity) of the p65 subunit. (H) Representative images in each condition. Experiments from F-H evaluated 10,000 cells. All results represent mean \pm SEM; ${ }^{*} p<0.05,{ }^{* *} p<0.005$, $* * * p<0.0005$. Reprinted with permission. Goldsmith ZK, Coppess W, Irvine AS, et al. Targeting the Platelet-Derived Growth Factor-beta Stimulatory Circuitry to Control Retinoblastoma Seeds. Invest Ophthalmol Vis Sci. 2018;59(11):4486-4495. Copyright (2018), with permission through Creative Common CC-BY. 


\section{Discussion}

Our findings demonstrate that PDGFR $\beta$ signaling promotes the growth of $\mathrm{Rb}$ seeds, reduces $\mathrm{Rb}$ tumor apoptotic activity, and controls their invasive potential. These results suggest the PDGFR $\beta$ signaling network may be a novel target to control $\mathrm{Rb}$, especially in settings with recurrent or recalcitrant vitreous disease. $\mathrm{Rb}$ is the most common primary intraocular malignancy in children. Although Rb is highly treatable in developed countries, treating eyes with vitreous seeds remains challenging. About a third of the patients who undergo eye-sparing therapies still require enucleation due to recalcitrant disease. This in part due to vitreous seeding, which is difficult to treat due to the anatomical location of the seeds and the refractory nature of them to the most commonly used chemotherapeutics. Therefore, understanding more about vitreous seeds and their microenvironment may prove advantageous in developing novel therapies against $\mathrm{Rb}$ seeds and lead to improved ocular salvage and visual preservation. Only recently has PDGFR $\beta$ signaling pathway in $\mathrm{Rb}$ come under scrutiny and been demonstrated in $\mathrm{Rb}$ samples by immunohistochemistry ${ }^{240}$ and showed in vitro the potential use of imatinib in reducing the growth of $\mathrm{Rb}$ prior to radiation using cell lines ${ }^{241}$. Our work confirmed the immunohistochemical studies by Burnier and demonstrated the PDGF-PDGFR $\beta$ signaling sustains $\mathrm{Rb}$ growth in both autocrine, growth factors stemming from the tumor cells, and paracrine, stemming from other, non-tumoral cellular infiltration, stimulatory loops. We demonstrated this stimulatory circuitry by the high expression of $P D G F B$ mRNA in Rb tumor cells, the levels of active PDGFR $\beta$ signaling, and the abundance of the PDGFR $\beta$ ligands, PDGF-AB and PDGF-BB, in the vitreous microenvironment of $\mathrm{Rb}$ patients.

Imatinib mesylate, aka as STI571, is marketed as Gleevec ${ }^{\circledR}$ by Novartis Pharmaceuticals Corporation. This compound belongs to the 2-phenylaminopyrimidine class drugs, which selectively inhibits the tyrosine kinase activity of the PDGFR, KIT, and BCR-ABL protein tyrosine kinases ${ }^{242,243}$. Considered one of the major breakthroughs in cancer treatments, IM lifted the death sentence associated with Chronic Myelogenous Leukemia (CML) after its first clinical study ${ }^{237}$, and is now indicated for multiple malignancies ${ }^{236,244-249}$. IM has shown antitumor activity as a neo-adjuvant in glioblastoma multiforme $(\mathrm{GBM})^{250}$. Similar to Rb, GBM shows high expression of PDGFRs. More importantly, these studies suggest a clinically safer application in the pediatric population and some penetration of IM to the Blood-Brain Barrier (BBB). The Blood-Retinal Barrier $(\mathrm{BRB})$ is considered the counterpart of the $\mathrm{BBB}$ in the retina. Emerging evidence shows the use of IM in reducing neuroinflammation in autoimmune disorders that target the central nervous system, such as multiple sclerosis (MS) ${ }^{251,252}$. In addition to the amelioration in neuroinflammation, IM restores the integrity of the BBB in a rat model of $\mathrm{MS}^{251}$. Together, these investigations became part of our rationale to investigate the PDGFR $\beta$ signaling pathway in Rb by using IM.

Vascular endothelial growth factor (VEGF) is abundant under pathological conditions. VEGF plays a role in tumor angiogenesis and the neutralization of this protein can reduce tumor vascularization in vivo. Prior work has demonstrated the use of bevacizumab, a monoclonal antibody against VEGF, in the suppression of angiogenesis 
and $\mathrm{Rb}$ growth in vitro using $\mathrm{Y} 79$ cells ${ }^{166}$. The use of bevacizumab in vivo also reduced tumor burden ${ }^{166}$. To achieve a reduction in tumor burden, the authors injected intraperitoneally $50-100 \mu \mathrm{g}$ of bevacizumab twice a week for 4 weeks to xenografted mice. This and other studies suggested anti-angiogenic therapy by inhibition of VEGF might be a suitable therapy against $\mathrm{Rb}^{166,253}$. However, recent work demonstrated that anti-VEGF therapy does not affect $\mathrm{Rb}$ cell viability and is not $\mathrm{Rb}$ specific ${ }^{254}$. We postulate PDGF-PDGFR signaling may contribute to VEGF production in the tumor microenvironment, as evidenced by the reduction in VEGFR2 signaling in Rb cell lines after disruption of the PDGFR $\beta$ signaling pathway.

That we know very little regarding vitreous seeds (and even less about the microenvironment) has precluded the advancement in the development of novel therapies against $\mathrm{Rb}$ vitreous seeds. We generated a novel $3 \mathrm{D}$ cell culture system based on the magnetic levitation studies to develop $\mathrm{Rb}$ tumor seeds in vitro ${ }^{255}$. When these spheroids were examined morphologically, they were similar to in vivo Rb vitreous seeds. The three main subtypes of vitreous seeding were recently outlined patterns: dust, spheres, and clouds ${ }^{100}$. Each of the subtypes results in different characteristics and potentially treatment responses. Furthermore, the histopathology associated with each subtype and concluded spheres to be the most aggressive subtype ${ }^{101}$. We compared our in vitro spheroids to the morphological characteristics previously described ${ }^{101}$ and discovered similarities to the spheres subtype. A translucent center was observed in the spheroids and some spheroids exhibited $\mathrm{Rb}$ cell detachment from the outer area. These studies validated our in vitro 3D system for the further study of $\mathrm{Rb}$ spheroids.

In this investigation we focused on the downstream signaling components of the PDGFR $\beta$ in Rb biology. We discovered the PDGFR $\beta$ signaling is active in ocular tissues, including the vitreous, from $\mathrm{Rb}$ patients compared to healthy controls. These results were confirmed using a PDX model, which have comparable molecular features to primary tumors. We also demonstrated the PDGFR $\beta$ signaling cascade is pivotal for Rb cell growth, survival and invasion. In an important step toward the successful evolution from translational research into future clinical applications, we generated a 3D cell culture system to study $\mathrm{Rb}$ spheroids. These spheroids have similar morphological features to the $\mathrm{Rb}$ sphere subtype of vitreous seeds. This cell culture system provides a unique opportunity to study the interactions between $\mathrm{Rb}$ and the microenvironment in vitro as it mimics the natural physiology by production of components of the extracellular matrix and growth factors. This study demonstrated pre-clinically that PDGF from both autocrine and exogenous sources signals through the PDGFR $\beta$ to sustain $\mathrm{Rb}$ growth in an avascular system, such as the vitreous. Targeting the PDGFR $\beta$ could increase the sensitivity of these tumor cells to current treatments as current therapies have failed to address the reduced proliferative capacity and metabolisms of these unique tumor seeds.

Following these studies, further work must be conducted in vivo to properly assess the therapeutic value of targeting the PDGF-PDGFR $\beta$ signaling pathway as a novel target to treat vitreous seeding. As this work is aimed at vitreous seeds, it only seems appropriate to conduct this future work using the rabbit xenograft model; this model has multiple features that make it an ideal modeling system: 1) the rabbit xenograft 
is the only model that readily forms vitreous seeds, 2) the size and volume of the eye are approximate to that of a small child, and 3) this model can withstand multiple injections, similarly to what is used within the clinic now. Preclinical assessment of anti-

PDGFR $\beta$ therapy should be done following injection and vitreous seed formation (approximately 6-8 weeks) primarily using fundus imaging to check for presence (or absence of vitreous seeds) as well as histopathology to examine vitreous seed phenotype and activation status of PDGFR $\beta$. Depending on the success of this study, future experiments can test other therapeutic avenues to disrupt PDGF-PDGFR $\beta$ signaling including neutralizing antibodies against PDGF-BB, aptamers, RNA interference, etc. As an alternative, additional studies should be aimed at examining the role of antiPDGFR $\beta$ therapy as an adjuvant therapy to sensitize vitreous seeds.

\section{Conclusion}

The work presented herein demonstrates the abundance of key PDGF ligands and the activation status of PDGFR $\beta$ as they relate specifically to vitreous seeds. We demonstrated PDGF-PDGFR $\beta$ signaling regulates key tumor cell behaviors including proliferation, invasion, and, most notably, survival. Moreover, we provide mechanistic insight that this survival is driven by the downstream targets of PDGFR $\beta$ signaling, most notably the anti-apoptotic molecules MDM2 and BCL-2 as well as the pro-survival molecule NFkB. We also developed the first in vitro model of vitreous seeds based on magnetic levitation. This model can further our understanding of vitreous seeds and supplies a much-needed preclinical model to test therapeutic efficacy. Nonetheless, further in vivo experiments are needed to evaluate the therapeutic value of PDGFR $\beta$ therapies. Taken together, the work presented here demonstrates a highly translational approach to successfully identify and inhibit a new potential target in PDGFR $\beta$ for vitreous seeding. These results point to the possibility of PDGFR $\beta$ inhibition as an adjuvant therapy for $R b$. 


\section{CHAPTER 4. RESULTS III: ANTI-PLATELET-DERIVED GROWTH THERAPY AS A POTENTIAL TARGET TO DECREASE RESISTANCE}

\section{Introduction}

Within the field of oncology, there has been a recent shift from the understanding of tumors as independent actors to that of a complex web of interactions featuring multiple cytokines and cell types, called the tumor microenvironment (TME). Interactions between the tumor and the TME are capable of altering fundamental processes of tumors including growth, survival, metastasis, and resistance. Interest in the TME has grown exponentially due to the possibility of new, more targeted therapies. As our lab is primarily interested in developing new therapies to target and clear vitreous seeds to increase ocular salvage rates, understanding more about the Rb TME is essential in meeting the treatment goals of $\mathrm{Rb}$. Unfortunately, vitreous seeding remains the most challenging aspect of treating and managing $\mathrm{Rb}$. Quite often these seeds are refractory to chemotherapy and thus lead to disease recurrence. How? and why? these seeds are refractory is not understood as very little is currently known about the Rb TME. Previous attempts have demonstrated a number of cytokines and growth factors within the vitreous that play a role in ocular pathologies ${ }^{225,226}$ however these studies are not directly related to $\mathrm{Rb}$ or vitreous seeding. Studies aimed at demonstrating other cell types within the TME of retinoblastoma $(\mathrm{Rb})$ have detected the presence of two non-tumor cell types: 1 ) macrophages ${ }^{105,256}$ and retinal vascular endothelial cells ${ }^{257}$. This gap in knowledge has resulted in a failure to raise ocular salvage rates over the past 20 years ${ }^{218-221}$.

Previous Rb studies aiming at vascular endothelial growth factor (VEGF) have failed to achieve clinical success ${ }^{166,258}$. Furthermore, the vitreous lacks vasculature so anti-angiogenic therapy should not aid in the clearance of vitreous seeds. To potentially target vitreous seeds, we recently identified the Platelet-Derived Growth Factor Receptorbeta (PDGFR $\beta$ ) as being highly active in Rb disease and vitreous ${ }^{216}$. We targeted the PDGF-PDGFR $\beta$ signaling pathway using imatinib mesylate (IM, aka Gleevec ${ }^{\circledR}$ from Novartis) and showed in vitro reduction of Rb growth, invasion, and survival in an MDM2- and NF-кB-dependent manner. While we demonstrated early, preclinical success of anti-PDGF-PDGFR $\beta$ therapy, we were also interested in how PDGF-PDGFR $\beta$ signaling may represent a potential mechanism by which vitreous seeds can evade cell death in response to chemotherapy.

Drug resistance is a major problem in cancer biology, whereby tumor cells no longer to respond to certain treatments (most commonly chemotherapy). There are multiple mechanisms that confer resistance to tumor cells including: 1) drug efflux, or the removal of intracellular drugs from the cell; 2) genetic responses promoting expression of resistance genes; 3) enhanced DNA repair to prevent programmed cell death; 4) alterations in tumor molecules that promote alternative activation of similar pathways; and 5) changes in metabolic pathways that result in decreased drug concentrations ${ }^{259}$. Cooperation between stromal cells and tumor cells have also been found to decrease drug efficacy through secretion of growth factors such as VEGF and PDGF ${ }^{260,261}$. Ultimately, 
cells that are drug resistant are not only more difficult to treat but are often more aggressive, more likely to metastasize, and give rise to disease recurrence. Perhaps the most well studied area in drug resistance is that of drug efflux where cells efflux, or pump, intracellular agents (i.e. chemotherapy) out of the cell resulting in less cytotoxic concentrations of chemotherapy. The ATP-binding cassette (ABC) transporter family is a group of efflux pumps that have been implicated in cancer drug resistance ${ }^{262-264}$.

The objectives of this study are two-fold: first, to investigate in vitro if other components of the TME would be affected by the PDGF-PDGFR $\beta$ pathway and IM toxicity, and second, to investigate if this signaling pathway would exert an effect in known mechanisms of chemoresistance.

\section{Materials and Methods}

\section{Ethics statement}

As stated in previous chapters, all experiments involving human subjects were approved by the Institutional Review Board (IRB) at St. Jude Children's Research Hospital (SJCRH) and The University of Tennessee Health Science Center (UTHSC); informed consent obtained when needed. In those experiments using tissue from murine xenografts, the Institutional Animal Care and Use Committee (IACUC) at SJCRH approved the animal experiments. All protocols followed the ARVO Statements for the use of animals in vision research, in addition to the guidelines for laboratory animal experiments.

\section{Cell lines and cell culture conditions}

Retinoblastoma cell lines. The human Retinoblastoma $(\mathrm{Rb})$ cell lines $\mathrm{Y} 79$ $\left(\text { ATCC }^{\circledR} \text { HTB- } 18^{\mathrm{TM}}\right)^{79}$ was purchased from the American Type Culture Collection (Manassas, VA, USA) and cultured in Rb media. This media contains RPMI-1640 supplemented with L-glutamine (Gibco by Invitrogen, Carlsbad, CA, USA), 20\% FBS (Gibco by Invitrogen), and 1\% penicillin/ streptomycin (Gibco by Invitrogen).

Breast cancer cell line. Metastatic breast cancer cells MDA-MB-231 $\left(\right.$ ATCC $^{\circledR}$ HTB-26) ${ }^{265}$ were a gift from Dr. T.J. Hollingsworth (UTHSC) and maintained in DMEM supplemented with 10\% FBS (Gibco by Invitrogen) and 1\% Amphotericin B / Gentamicin. Cells were kept in a humidified incubator at $37^{\circ} \mathrm{C} / 5 \% \mathrm{CO}_{2}$.

\section{Primary human retinal microvascular endothelial cells (hRECs, ACBRI}

181). These cells were purchased from Applied Cell Biology Research Institute. hRECs were cultured using Complete Classic Medium was used, consisting of Complete SerumFree Medium with 10\% HI-FBS (Gibco by Invitrogen), and 1\% penicillin/ streptomycin (Gibco by Invitrogen). ACBRI181 were used for experiments before passage 8 . 
Cell culture conditions. Cells were cultured using four different conditions: untreated, recombinant human Platelet-Derived Growth Factor (rhPDGF, 10ng/mL), Imatinib Mesylate $(\mathrm{IM}, 10 \mu \mathrm{M})$, and the combination of rhPDGF + IM.

\section{Vitreous samples}

The University of Tennessee Health Science Center and St. Jude Children's Research Hospital Institutional Review Board (IRB) approved the present study. Informed consent was obtained in all cases where vitreous was harvested at the time of patient enucleation, an optional research objective that is part of an on-going prospective clinical trial (NCT01783535) This is in full compliance with and adheres to the tenets of the Declaration of Helsinki and the ARVO statement on human subjects. Vitreous from eyes with $\mathrm{Rb}$ was collected at the time of enucleation. Eyes were trephined immediately upon removal without disruption of underlying structures important for diagnostic and staging purposes. Tumor was harvested for genetics and biology and vitreous was aspirated using an 18-gauge needle and $3 \mathrm{~mL}$ syringe. Vitreous from healthy controls were purchased from BioreclamationIVT (Baltimore, MD, USA). Samples were centrifuged, aliquoted and stored at $-20^{\circ} \mathrm{C}$.

\section{Reagents}

Phosphate-buffered saline (PBS) was purchased from Gibco by Invitrogen (Thermo Scientific, Bridgewater, NJ, USA). Gleevec ${ }^{\circledR}$ was purchased in its generic chemical form, imatinib mesylate (IM, PubChem ID: 123596), from Sigma-Aldrich (Saint Louis, MO, USA). Lyophilized reagent was dissolved in deionized, distilled water $\left(\mathrm{ddH}_{2} \mathrm{O}\right)$ to $10 \mathrm{mM}$, aliquoted, and stored at $-20^{\circ} \mathrm{C}$. Lyophilized recombinant human PDGF-BB $(10 \mu \mathrm{g})$ was purchased from Invitrogen (Thermo Scientific), dissolved in acetic acid, aliquoted, and stored at $-20^{\circ} \mathrm{C}$.

\section{MTS cell proliferation assay}

Cell proliferation studies were performed using the CellTiter $96^{\circledR} \mathrm{AQ}_{\text {ueous }}$ One Solution Cell Proliferation Assay (Promega, Madison, Wisconsin, USA) as before ${ }^{170}$. Briefly, $1.0 \times 10^{4} \mathrm{hREC}$ cells per well were cultured in the conditions described above. CellTiter reagent was added at a concentration of $20 \mu \mathrm{L}$ per $100 \mu \mathrm{L}$ volume per well at specific time points of 0-, 48-, 72-, and $96 \mathrm{~h}$ after culture. Cells were incubated at $37^{\circ} \mathrm{C}$ for $2 \mathrm{~h}$ before absorbance was read at $490 \mathrm{~nm}$ using a $96-$ well plate reader. Values expressed as mean \pm SEM, $n=3$ with 4 replicates. Statistical analysis done using Prism Graph Pad. 


\section{qPCR analysis}

RNA isolation. RNA from Y79 Rb cells, hRECs, and each vitreous sample were extracted following the Qiagen ${ }^{\circledR}$ miRNeasy Mini Kit (Qiagen, Valencia, CA) manufacturer's recommendations. $\mathrm{Rb}$ cells were harvested and centrifuged to remove all traces of cell culture medium. Cell pellets were lysed and homogenized prior to addition of chloroform. Samples were shaken vigorously for $15 \mathrm{~s}$ prior to centrifugation for $2 \mathrm{~min}$ at full speed. The upper colorless phase was transferred to a clean tube after centrifugation followed by a series of alcohol precipitations to provide ideal binding conditions. The lysate was loaded onto an RNeasy spin column with a silica membrane to bind the RNA and washed away contaminants. Residual DNA was removed by oncolumn DNase digestion. The concentrated RNA was eluted in $15 \mu \mathrm{L}$ of RNAse free water. RNA concentration was assessed by analysis on Nanodrop 2000 Spectophotometer (ThermoFisher Scientific) and purity was assessed by verifying absorbance ratios of $260 \mathrm{~nm}$ and $280 \mathrm{~nm}$ (A260/A280). Samples with absorbance ratios between 1.8 and 2.0 were considered free of contamination. For more information, please see Table A-1.

cDNA synthesis and pre-amplification. Synthesis of cDNA was performed using the SuperScript ${ }^{\mathbb{R}}$ VILO ${ }^{\text {TM }}$ cDNA Synthesis Kit (Life Technologies, Grand Island, NY). Following manufacturer's directions, we used 100ng of isolated RNA (see section above) and combined them with $4 \mu \mathrm{L} 5 \mathrm{X}$ VILO Reaction Buffer and $2 \mu \mathrm{L} 10 \mathrm{X}$ SuperScript Enzyme Mix. Final volume per sample was $20 \mu \mathrm{L}$ using DEPC-treated water when necessary. Per manufacturer's directions, cDNA synthesis reaction was done at $25^{\circ} \mathrm{C}$ for $10 \mathrm{~min}$ followed by $42^{\circ} \mathrm{C}$ incubation for $60 \mathrm{~min}$ with reaction termination at $85^{\circ} \mathrm{C}$ for $5 \mathrm{~min}$. Material was pre-amplified using $10 \mu \mathrm{L} 2 \mathrm{X}$ TaqMan ${ }^{\circledR}$ PreAmp Master Mix, $5 \mu \mathrm{L}$ pooled $0.2 \mathrm{X}$ TaqMan ${ }^{\circledR}$ primers, and $5 \mu \mathrm{L}$ cDNA sample for a final volume of $20 \mu \mathrm{L}^{192}$. Preamplification reactions were conducted according to manufacturer's guidelines with a $10 \mathrm{~min}$ enzymatic activation step at $95^{\circ} \mathrm{C}$ followed by 14 cycles of denaturing $\left(95^{\circ} \mathrm{C}\right.$ for $\left.15 \mathrm{~s}\right)$ and annealing $\left(60^{\circ} \mathrm{C}\right.$ for $\left.4 \mathrm{~min}\right)$. Following 14 cycles, enzyme was inactivated at $99^{\circ} \mathrm{C}$ for $10 \mathrm{~min}$ and reactions were held at $4^{\circ} \mathrm{C}$ until use.

Preamplification utilized the same primers analyzed to use minimal amounts of material while increasing sensitivity of detection. The reaction was kept at $-20^{\circ} \mathrm{C}$ until ready to use.

PCR. We used the following Human TaqMan ${ }^{\circledR}$ Gene Expression Assays: HPRT1 (Hs02800695 m1), PDGFRB (Hs01019589 m1), FLT1 ( $\mathrm{Hs} 01052961 \mathrm{m1}), A B C B 1$ (Hs00184500_m1), ABCC1 (Hs01561483_m1), $A B C C 2$ (Hs00960489_m1), ABCG2 (Hs01053790_m1), CD44 (Hs01075864_m1) all from Life Technologies (Grand Island, NY). A final volume of $10 \mu \mathrm{L}$ was loaded into each well after combination of $5 \mu \mathrm{LTaqMan}{ }^{\circledR}$ Universal Master Mix, $2.5 \mu \mathrm{L}$ cDNA, $0.5 \mu \mathrm{Lprimers}$ and $2.0 \mu \mathrm{L}$ Nuclease-Free water. White, 96 -well PCR plates were run using Roche ${ }^{\circledR}$ LightCycler 480 using TaqMan ${ }^{\circledR}$ protocol: reaction was initiated at $50^{\circ} \mathrm{C}$ for $2 \mathrm{~min}$ followed by $95^{\circ} \mathrm{C}$ for $10 \mathrm{~min}$ before $40 \mathrm{PCR}$ cycles were conducted at $95^{\circ} \mathrm{C}$ for $15 \mathrm{~s}$ and $60^{\circ} \mathrm{C}$ for $1 \mathrm{~min}$. Values greater than 35 were excluded due to low confidence. Data was analyzed using the Comparative $\mathrm{C}_{\mathrm{T}}\left(\Delta \Delta \mathrm{C}_{\mathrm{T}}\right)$ Method where $\Delta \mathrm{C}_{\mathrm{T}}$ is the difference between gene-of-interest (GOI) and housekeeping gene (HKG) and $\Delta \Delta \mathrm{C}_{\mathrm{T}}$ is the 
difference between experimental (i.e. PDGF, IM, or PDGF+IM) and untreated. Fold change is determined by 2 raised to the negative $\Delta \Delta \mathrm{CT}$ value $\left[2^{(-\Delta \Delta \mathrm{CT})}\right]^{192,193}$. For more information, please see Table A-1.

\section{Tube formation assay}

hRECs were cultured on Reduced Growth-Factor Matrigel (BD Biosciences, Bedford, MA) in complete media (10\% FBS, Cell BioSystems) or low serum $(0.2 \%$ FBS). Additional culture conditions included rhPDGF and IM. Images were taken at both 6- and 18h using a Nikon C1 confocal microscope using $4 \mathrm{X}$ objective. Results are representative of two independent experiments; three fields were taken per group at both 10X (data not shown) and $4 \mathrm{X}$.

\section{Flow cytometry analyses}

Rb cell:hREC co-cultures. Monocultures of Y79 Rb, hRECs and co-cultures of Y79 Rb and hRECs (Rb:hREC) were treated using recombinant human Platelet-Derived Growth Factor (rhPDGF, 10ng/mL), Imatinib Mesylate (IM, 10 $\mu \mathrm{M}$ ), and the combination of rhPDGF + IM. Cell cultures were harvested at 24h, fixed in PBS / 2\% paraformaldehyde, and labeled with anti-human PDGFR $\beta$ APC or with anti-VEGF-A AF700 (BioLegend). Data acquisition was performed using a Bio-Rad ZE5 Cell Analyzer (aka YETI, Propel Labs, Fort Collins, CO); analysis done using FlowJo vX.0.5 (Tree Star). For more information, please see Table A-2.

Assessment of efflux activity. The activity of multidrug resistance proteins was analyzed by eFLUXX-ID ${ }^{\circledR}$ Green multidrug resistance assay kit (ENZ-51029-K100, ENZO Lifesciences, New York, NY, USA) by manufacturer's suggested instructions. Briefly, $4.0 \times 10^{6}$ Y79 cells were plated and treated with either rhPDGF or IM for $24 \mathrm{~h}$. After 24h, cells were harvested, washed, and resuspended in full media containing respective MDRP inhibitors at $37^{\circ} \mathrm{C}$ for $10 \mathrm{~min}$. eFLUXX-ID ${ }^{\circledR}$ Green dye was then added and cells were incubated for an additional $30 \mathrm{~min}$ at $37^{\circ} \mathrm{C}$. Data acquisition was done in a ZE5 Cell Analyzer (aka YETI), from Propel Labs (Fort Collins, CO, USA). Analysis was done using FlowJo software v.X.0.0.8 (Tree Star).

Assessment of co-expression of the pPDGFR $\boldsymbol{\beta}$ and CD44 proteins. Y79 Rb cells cultured in the conditions described above were permeabilized with Intracellular Staining Perm and Wash Buffer (BioLegend, San Diego, CA, USA) and labeled with polyclonal rabbit anti-p-PDGFR $\beta$ (Cell Signaling Technology, \#4549; 1:50) as well as anti-human CD44 PerCP/Cy5.5 (BioLegend, San Diego, CA, USA) antibodies on ice for $30 \mathrm{~min}$ followed by incubation with an anti-rabbit Alexa Fluor ${ }^{\circledR} 488$ (Thermo Scientific, $1: 50)$ secondary antibody for $30 \mathrm{~m}$. Cells were then fixed with $2 \%$ paraformaldehyde (ThermoFisher) for 20min prior to analysis. Data acquisition was done in a ZE5 Cell Analyzer ( $a k a$ YETI), from Propel Labs (Fort Collins, CO, USA). Analysis was done 
using FlowJo software v.X.0.0.8 (Tree Star). For more information, please see Table A-2.

\section{Results}

\section{Proliferation of primary human retinal endothelial cells is independent of PDGF- PDGFR $\beta$ signaling}

Prior work from members of our collaborative team have demonstrated how chemotherapy can potentially induce ocular toxicities via inflammation and leukostasis resulting in hREC death. Therefore, we tested whether disruption of PDGF-PDGFR $\beta$ signaling or an off-target effect of IM can induce similar effects in vitro. As we previously demonstrated an abundance of active PDGFR $\beta$ (p- PDGFR $\beta$ ) in Rb (Figure 3-1), we hypothesized targeting PDGF-PDGFR $\beta$ would be tumor specific and, therefore, less toxic to hRECs. To explore this hypothesis, we first measured Y79 proliferation using the tyrosine kinase inhibitor (TKI) imatinib mesylate (IM), a known inhibitor of cKit, Bcr-abl, and PDGFRs. We had previously identified $10 \mu \mathrm{M}$ as the most efficacious concentration $^{216}$. Inhibiting the PDGFR $\beta$ signaling with IM significantly reducing proliferation of Y79 Rb cells at 72h (Figure 4-1A). As a physiological control, we stimulated Y79 Rb cells with recombinant human PDGF-BB (rhPDGF), which we discovered to be highly abundant in the tumor microenvironment of $\mathrm{Rb}$ by examination of $\mathrm{Rb}$ patient vitreous samples. To investigate what role PDGF-BB may have on hRECs and to test if IM may be toxic to hRECs, we cultured hRECs in the same conditions as Y79 Rb cells. We tested proliferation over 96h using MTS cellular proliferation assay and measured no changes in cellular proliferation (Figure 4-1B). These results illustrate how hREC proliferation occurs independent of PDGF-PDGFR $\beta$ signaling. Next, we investigated the expression levels of PDGFR in hRECs as a means to measure PDGFR $\beta$ activity. We harvested mRNA from hRECs and Y79 cells to measure the expression of FLT1 (VEGFR1) and PDGFRB relative to HPRT1, a housekeeping gene. The mRNA expression of FLT1 was higher in hRECs compared to Y79 Rb cells. However, PDGFRB mRNA expression was not detected (Figure 4-1C) in hRECs, while high expression was uncovered in Y79 Rb controls. Taken together, these results demonstrate how targeting of PDGF-PDGFR $\beta$ signaling should not have anti-proliferative effects in hRECs, as they do not express $P D G F R B$.

\section{Disruption of the PDGF-PDGFR signaling pathway does not affect angiogenic activity in hRECs}

As we did not determine any changes in proliferation and noted a lack of PDGFR $\beta$ mRNA expression in hRECs, we hypothesized stimulation with rhPDGF would not promote hREC organization or tube formation. Furthermore, we hypothesized inhibition of PDGF-PDGFR $\beta$ by IM would similarly fail to have an effect on tube 

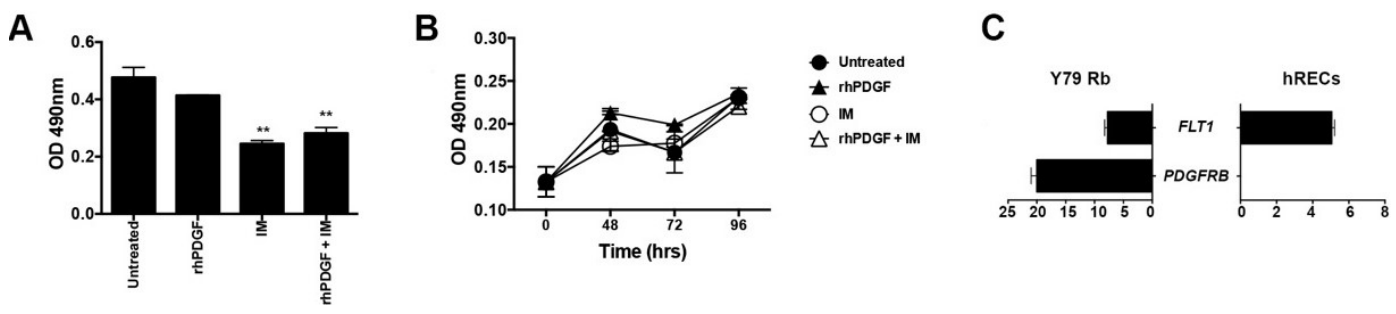

Figure 4-1. hREC proliferation occurs independent of PDGF-PDGFR $\beta$ signaling.

Y79 cells or hRECs were cultured in the presence or absence of either PDGF-BB (10ng/mL) or the PDGFR $\beta$ inhibitor imatinib mesylate (IM, 10 $\mu \mathrm{M})$. (A) Assessment of Y79 cellular proliferation at $72 \mathrm{~h}$ the presence of either PDGF-BB or IM using the concentrations described above. (B) Assessment of hREC proliferation over $96 \mathrm{~h}$ in the presence of either PDGF-BB or IM using the concentrations described above. (C) qPCR analysis was done on both cell types to measure mRNA levels of both FLT1 and PDGFRB. All experiments done with $\mathrm{N}=3$ and each sample done in triplicates. All results in represent mean $\pm \mathrm{SEM} ; * *<0.005$. 
formation. To test this hypothesis that anti-PDGFR $\beta$ treatment will not affect angiogenic activity of hRECs, we cultured hRECs on reduced-growth factor extracellular matrices under the following conditions: complete media (with 10\% FBS), low serum $(0.2 \% \mathrm{FBS})$, and low serum + rhPDGF $(10 \mathrm{ng} / \mathrm{mL})$. Non-pathogenic organization of hRECs display complex tubular structures similar to blood vessels (Figure 4-2, right). Under stressful conditions, simulated through serum starvation, hRECs do not readily organize into these branched structures, as shown in Figure 4-2, left. Next, we tested if the PDGF-PDGFR $\beta$ signaling could restore hRECs ability of tube formation through addition of exogenous rhPDGF. Results in Figure 4-2, middle demonstrate this signaling pathway cannot rescue hRECs angiogenic activity in vitro, defined by the tube formation assay.

As a next step, we tested if the PDGF-PDGFR $\beta$ signaling pathway is capable of promoting or stimulating tube formation. hRECs were all cultured in complete media and under the following conditions: untreated, stimulated with rhPDGF $(10 \mathrm{ng} / \mathrm{mL})$ or IM $(10 \mu \mathrm{M})$. We found no morphological changes in hREC vascular organization (Figure 4-3). Furthermore, to test if Rb cell secretions (i.e. PDGFs, VEGFs) could promote angiogenesis, hRECs were cultured in the presence of Y79 conditioned-media (CM). When cultured in the presence of CM, we found, similarly, tube formation of hRECs did not change (data not shown). Ultimately, these results indicate hRECs angiogenic function is independent of PDGF-PDGFR $\beta$ signaling.

\section{Reduction in hREC VEGF production is PDGFR $\beta$-independent}

To evaluate cellular interactions and potential role(s) PDGF-PDGFR $\beta$ signaling may play in the $\mathrm{Rb}$ tumor microenvironment, we set up a co-culture system composed of Y79 Rb cells and hRECs using the same conditions described before. Y79 Rb cells were labeled with the non-toxic dye carboxyfluorescein succinimidyl ester (CFSE) to trace the cells during single cell analyses, while leaving the hRECs unlabeled (Figure 4-4A). Cocultures of $\mathrm{Rb}: \mathrm{hREC}$ were done using two different ratios, 1:1 and 1:10, to examine potential cell number-dependent roles. Labeling for PDGFR $\beta$, we found that IM reduced the number of PDGFR $\beta^{+}$cells in Y79 monocultures (Figure 4-4B, top left) while we further confirmed our previous mRNA analysis as hRECs did not express PDGFR $\beta$ (Figure 4-4B). In our co-cultures, we measured reductions in the percentage of cells expressing PDGFR $\beta$ after IM treatment (Figure 4-4B, top left).

Next, we examined the percentage of vascular endothelial growth factor-A (VEGF-A)-producing cells in both mono- and co-cultures after disruption of the PDGFPDGFR $\beta$ signaling pathway. In contrast to PDGFR $\beta$ expression, we measured reductions in the percentage of VEGF-A-producing cells in Y79 Rb and hRECs after treatment with IM (Figure 4-4C, bottom left). The reduction in the percentage of VEGF-A-producing cells was also demonstrated in the co-cultures (Figure 4-4C, bottom left). Collectively, our results show a reduction in Y79 Rb cells expressing PDGFR $\beta^{+}$and a reduction in VEGF-A-producing cells in both Rb tumor cells and hRECs. 

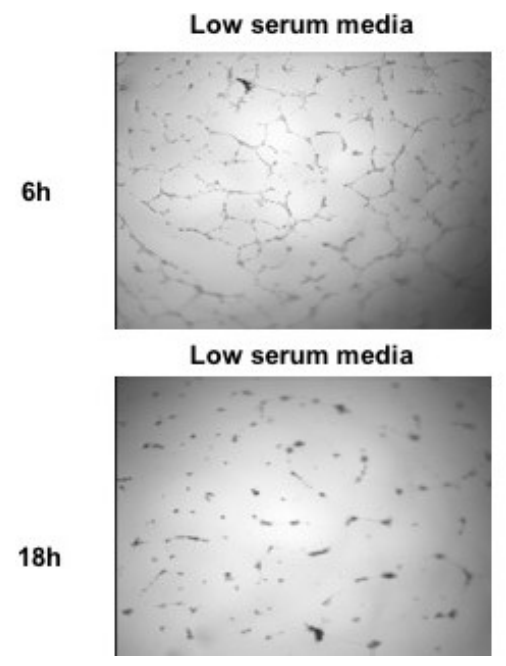

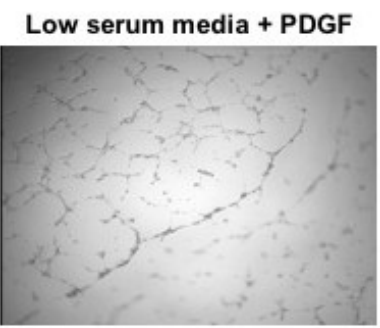

Low serum media + PDGF

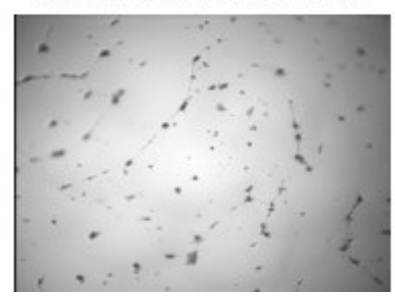

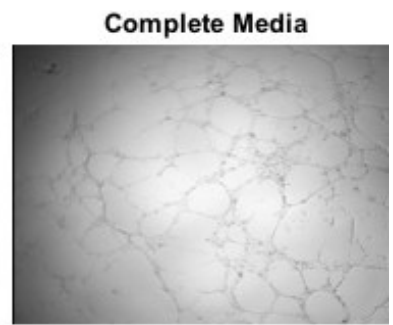

Complete Media

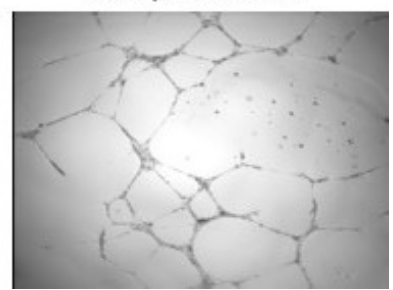

Figure 4-2. PDGF-PDGFR $\beta$ signaling is unable to initiate tube formation.

hRECs were serum starved overnight before plating on reduced growth factor Matrigel and treated with rhPDGF $(10 \mathrm{ng} / \mathrm{mL})$. Images were taken at $6 \mathrm{~h}$ and $18 \mathrm{~h}$ using a Nikon $\mathrm{C} 1$ microscope and 10X (above) and 4X (data not shown) objectives. All experiments done in quadruplicate. Images above are representative of field of view.

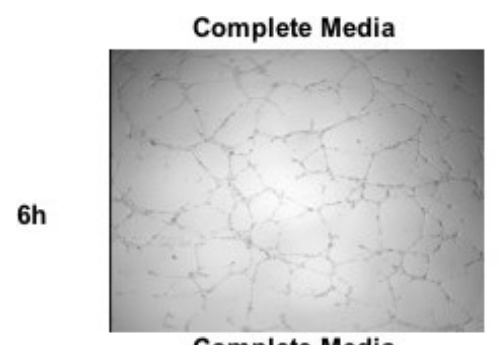

Complete Media

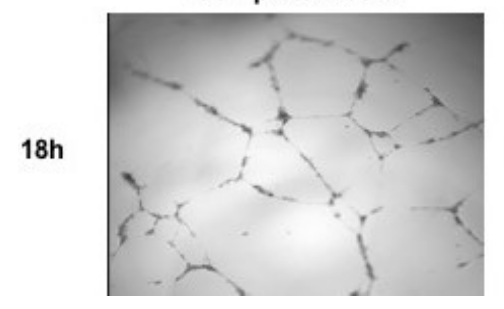

Complete Media + PDGF

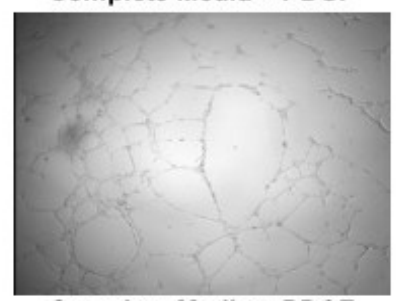

Complete Media + PDGF

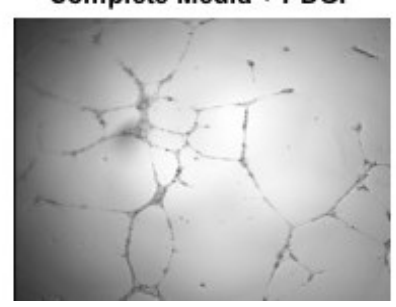

Complete Media + IM

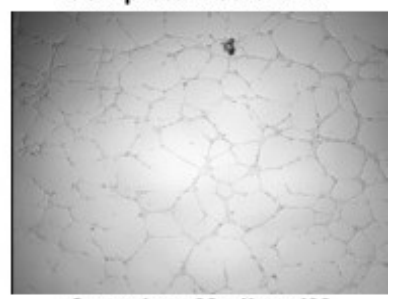

Complete Media + IM

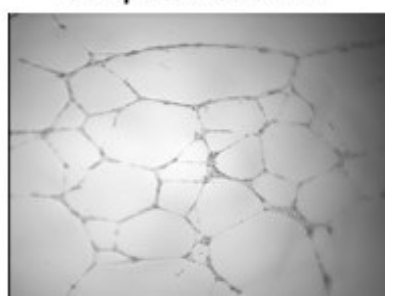

Figure 4-3. PDGF-PDGFR $\beta$ signaling is not required for hREC tube formation.

hRECs were serum starved overnight before plating on reduced growth factor Matrigel and treated with rhPDGF $(10 \mathrm{ng} / \mathrm{mL})$ or imatinib mesylate $(10 \mu \mathrm{M})$. Images were taken at $6 \mathrm{~h}$ and $18 \mathrm{~h}$ using a Nikon $\mathrm{C} 1$ microscope and 10X (above) and $4 \mathrm{X}$ (data not shown) objectives. All experiments done in quadruplicate. Images above are representative of field of view. 


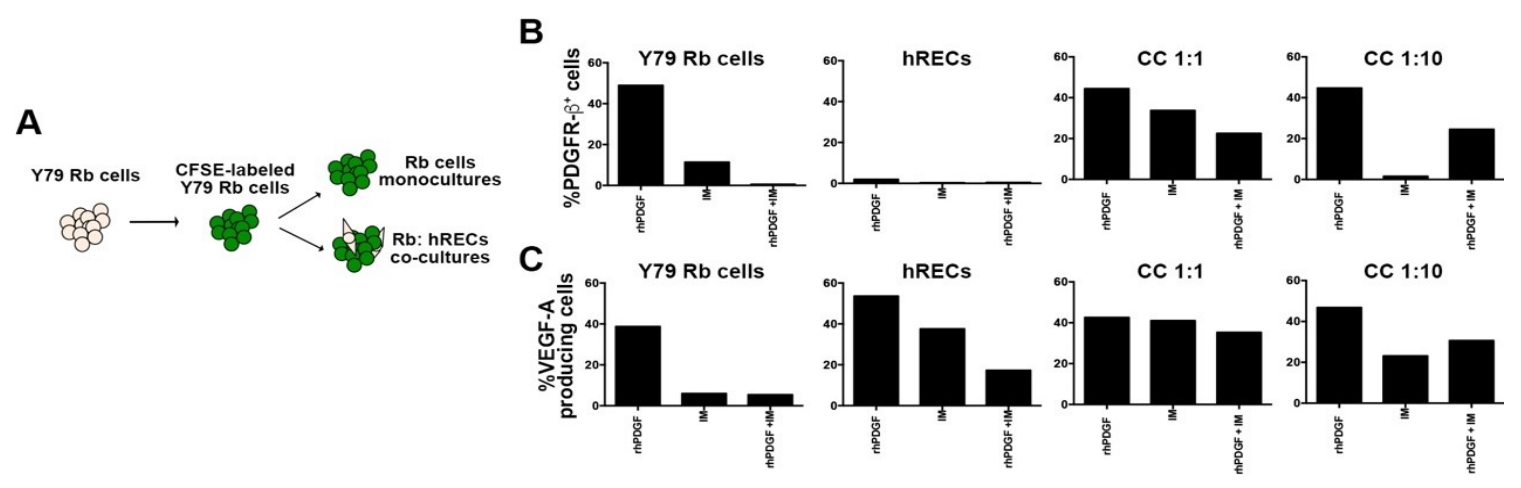

Figure 4-4. Treatment with IM reduces hREC VEGF production.

Y79 and hREC co-cultures were generated. (A) Schematic representation of experimental design. Y79 cells were labeled with CFSE to distinguish them from hRECs. Cells were plated alone or in specific ratios (Rb:hREC) to measure cellular interactions. All cultures were treated with rhPDGF $(10 \mathrm{ng} / \mathrm{mL})$ or imatinib mesylate $(10 \mu \mathrm{M})$. (B) Assessment of PDGFR $\beta^{+}$by flow cytometry. (C) Identifying the percentage of VEGF-A producing cells $\left(\mathrm{VEGF}-\mathrm{A}^{+}\right.$) using flow cytometry. $\mathrm{N}=2$ due to lack of hREC viability. 


\section{Expression of ATP-binding cassette (ABC) transporter family in the $\mathrm{Rb}$ vitreous and a tumor-derived cell line}

Members of the $\mathrm{ABC}$ transporters play essential roles in the development of chemoresistance. We followed up on previous studies ${ }^{266,267}$ examining ABC transporters in $\mathrm{Rb}$ by immunohistochemistry and hypothesized $\mathrm{ABC}$ transporters would be expressed in the vitreous of $\mathrm{Rb}$ patients. Moreover, if these previous studies were true, we would see heterogeneic expression of $\mathrm{ABC}$ transporters. To address this hypothesis, we first investigated the expression of four major ATP-binding cassette (ABC) transporters in the vitreous microenvironment of $\mathrm{Rb}$ samples from patients that were underwent primary enucleation (NCT01783535). The mRNA expression was compared to those of vitreous from healthy (purchased, BioreclamationIVT, Baltimore, $\mathrm{MD}$ ). Rb vitreous samples measured detectable expression of $A B C C 1$ (MRP1; 0.805 \pm 1.876 ), ABCC2 (MRP2; 2.407 \pm 1.021 ), and $A B C G 2$ (BCRP; 3.359 \pm 0.728 ) compared to the controls (Figure 4-5). The expression of $A B C B 1$ was quite variable $(0.805 \pm 1.876)$. These results help illustrate the heterogeneity of $\mathrm{Rb}$ tumors and, more specifically, the heterogeneity in expression of the $\mathrm{ABC}$ family in $\mathrm{Rb}$. To have an in vitro system we could use to study in more detail in lab, we assessed the mRNA expression of these genes in vitro using Y79 Rb cells. We detected expression of $A B C C 1$ and $A B C C 2$ while $A B C B 1$ and $A B C G 2$ were below detection threshold (Figure 4-6). Next, we evaluated the expression of these genes under stimulatory conditions and demonstrated that rhPDGF did not alter mRNA expression of these ABC transporters (Figure 4-6).

\section{Disruption of PDGF-PDGFR $\beta$ signaling reduces ABC transporter efflux}

The lack of retention of chemotherapy drugs inside tumor cells, or efflux, is one of the major obstacles to successful chemotherapy treatments. Prior preclinical studies demonstrated PDGF provided a tumor-specific efflux of chemotherapy and, when PDGFPDGFR $\beta$ signaling was inhibited, drug uptake was significantly improved ${ }^{268}$. Based on this study, we hypothesized PDGF-PDGFR $\beta$ signaling may promote drug efflux by MDRPs resulting in decreased intracellular chemotherapy concentrations that are below cytotoxicity threshold. To investigate this hypothesis, we quantified the ABC transporters efflux by chemical inhibition of the efflux pumps and measurement of an intracellular dye using the eFLUXX-ID ${ }^{\circledR}$ green multidrug resistance assay kit. This kit utilizes a cell permeant dye that, upon entrance intracellularly is cleaved by cellular esterases, and may only be effluxed by ABC transporters. Therefore, if drug efflux levels of Rb cells are high, this dye is pumped extracellularly, and fluorescence levels are low; on the other hand, if $\mathrm{Rb}$ cells have low efflux levels, this dye remains intracellularly, and cells maintain high amounts of fluorescence. Given the heterogeneic expression of MDRPs in $\mathrm{Rb}$ samples, we expect high levels of efflux (and therefore, low levels of fluorescence). Furthermore, we expect stimulation with rhPDGF to increase efflux activity (decreasing fluorescence further). We also expect treatment with IM to inhibit efflux, thereby increasing intracellular fluorescence. Surprisingly, we detected high amounts of intracellular fluorescence regardless of treatment condition (Figure 4-7). The results 


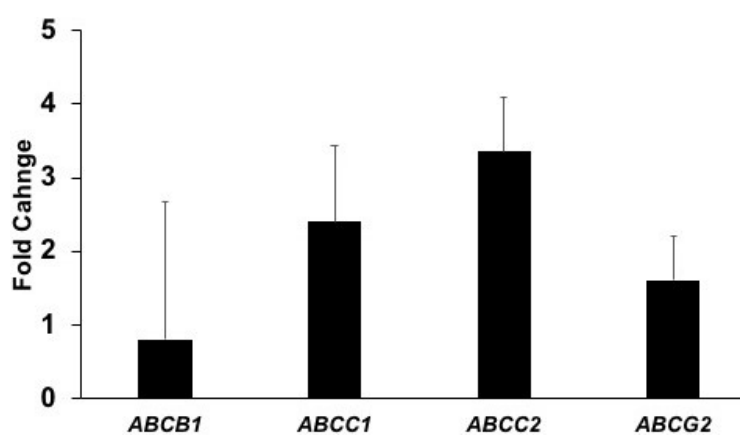

Figure 4-5. Expression of the ABC transporters in naïve human $\mathrm{Rb}$ vitreous.

qPCR analysis of mRNA isolated from ex vivo naïve Rb vitreous for key members of $\mathrm{ABC}$ transporter chemoresistance pathway. $\mathrm{N}=8$ with 4 replicates per sample. Bar graphs indicate results $\pm \mathrm{SEM}$ to control.

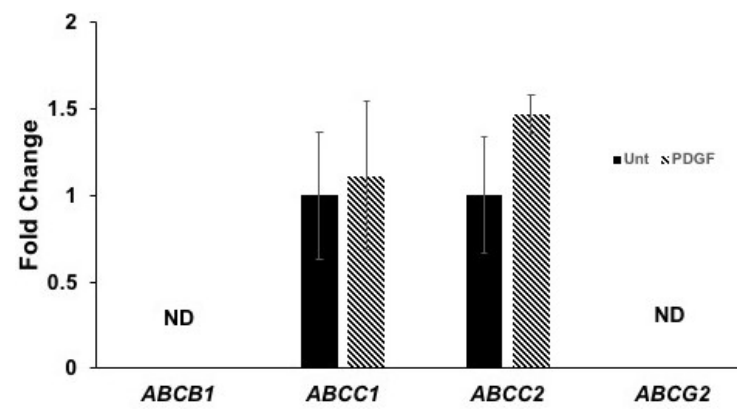

Figure 4-6. Expression of the $\mathrm{ABC}$ transporters in Y79 cell lines is not altered by PDGF-PDGFR $\beta$ signaling.

Y79 cells were stimulated for $24 \mathrm{~h}$ with $\mathrm{rhPDGF}(10 \mathrm{ng} / \mathrm{mL})$. After, RNA was isolated, cDNA was synthesized, and PCR was conducted. qPCR analysis of mRNA isolated from Y79 Rb cells for key members of $\mathrm{ABC}$ transporter chemoresistance pathway. $\mathrm{N}=4$ with 4 replicates per sample. Bar graphs indicate results \pm SEM to control. 


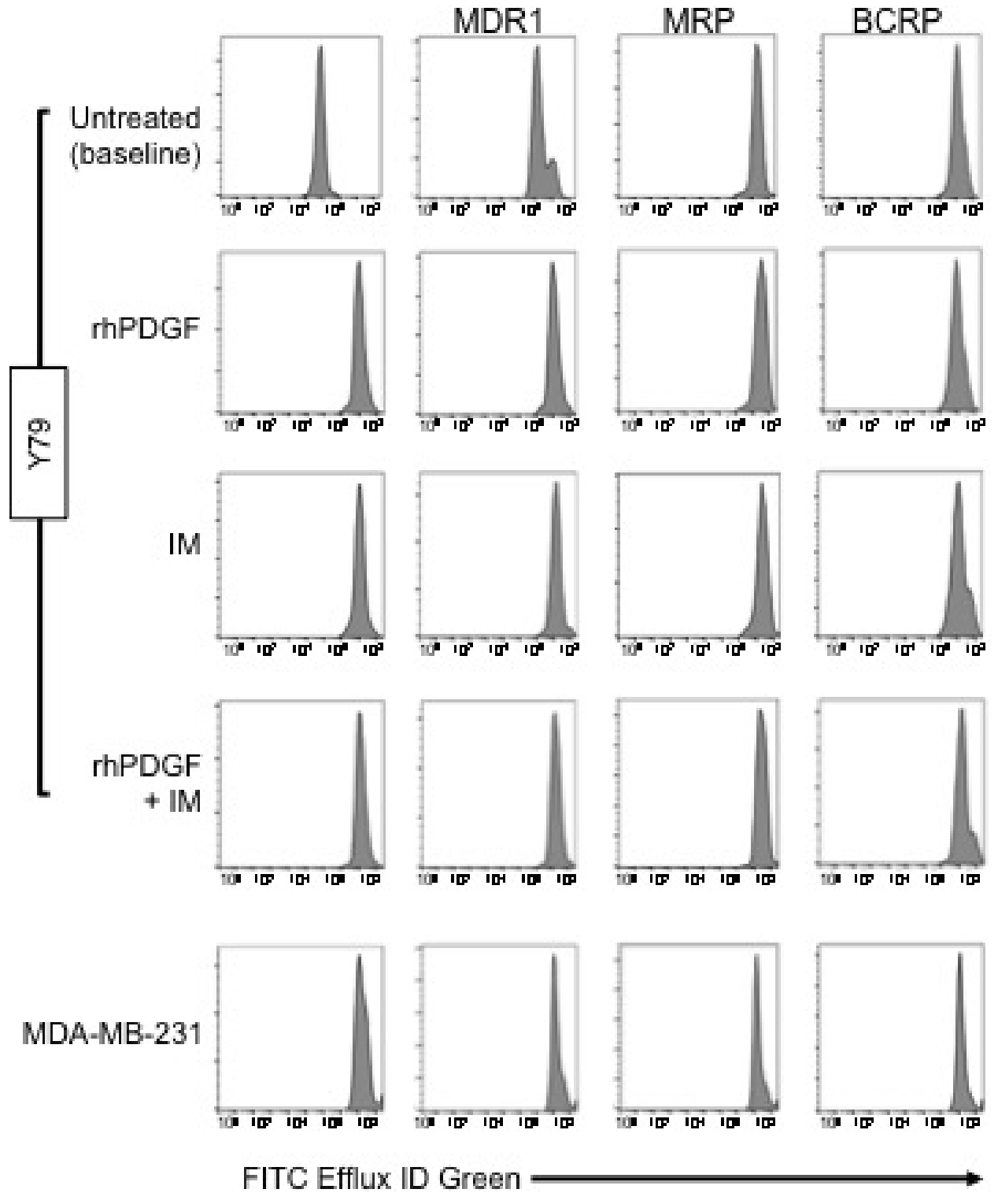

Figure 4-7, $\mathrm{ABC}$ transporter efflux activity is low in Y79 Rb cells.

Y79 cells were treated for $24 \mathrm{~h}$ with rhPDGF $(10 \mathrm{ng} / \mathrm{mL})$ or IM $(10 \mu \mathrm{M})$. After $24 \mathrm{~h}$, cells were collected and assayed using the eFFLUXX-ID ${ }^{\circledR}$ multidrug resistance assay kit. Flow cytometry histogram is shown. MDA-MB-231 cells were used a negative control.

Samples were immediately run on a BioRad ZE5. N=3 with 3 replicates per sample. 
changes in efflux activity in $\mathrm{Rb}$ cells. The results for $\mathrm{Y} 79$ were similarly to demonstrated the well-characterized and negative control metastatic breast cancer cell line, MDA-MB231 (Figure 4-7, right). This data illustrates that $\mathrm{Rb}$ does express $\mathrm{ABC}$ transporters at both mRNA and protein levels ${ }^{266,267}$; however, there may be a lack of functionality.

\section{CD44 is overexpressed in the naïve $R b$ vitreous and is by PDGF-PDGFR $\beta$ signaling}

The hyaluronan receptor, or CD44, has been heavily implicated in modulating of the resistance to chemotherapy by regulation of the interactions between tumor cells and the ECM. Therefore, $C D 44$ mRNA expression was investigated. We utilized ex vivo human $\mathrm{Rb}$ samples and measured a 40-fold increase in CD44 mRNA relative to HPRT1 in naïve $\mathrm{Rb}$ patients (Figure 4-8) that have not received any treatment. The results above further suggest drug resistance is an innate feature of vitreous seeds.

The overexpression of $C D 44$ may be dependent on the TME. Next, we tested if the PDGF-PDGFR $\beta$ signaling pathway may play a role in the overexpression of $C D 44$. $\mathrm{Y} 79 \mathrm{Rb}$ cells were treated with rhPDGF or IM and analyzed for the active form of the PDGFR $\beta$ (p-PDGFR $\beta$ ) and CD44. IM reduced the percentage of cells showing double positivity for $\mathrm{p}$-PDGFR $\beta^{+}$and $\mathrm{CD} 44^{+}$compared to rhPDGF stimulated cells (rhPDGF: $53.1 \%$ vs rhPDGF + IM: 35.4\%) (Figure 4-9). Also, the decrease in p-PDGFR $\beta^{+} \mathrm{CD} 44^{+}$ cells is enhanced by the combination of rhPDGF and IM conditions that mimic the TME (rhPDGF: $56.3 \%$ vs. rhPDGF + IM: 79.1\%). These results illustrate a potential role PDGF-PDGFR $\beta$ signaling may play in resistance by promoting a more resistant phenotype $\left(\mathrm{CD} 44^{+}\right)$.

\section{Discussion}

Retinoblastoma $(\mathrm{Rb})$ remains the most common intraocular malignancy afflicting children. While survival rates are above $90 \%$ in developed countries, ocular salvage rates have not readily exceeded $70 \%$ in over 2 decades primarily due to the presence of vitreous seeds. These seeds and their location within the vitreous make them exceedingly difficult to treat via systemic chemotherapy. To achieve higher, more tumoricidal doses, new mechanisms for drug delivery have been developed in recent decades, specifically those of super-selective intra-ophthalmic artery chemotherapy (IAC) and intravitreal chemotherapy (IVT) ${ }^{68,269}$. These new treatment mechanisms have garnered much attention due to their ability to increase ocular salvage by preliminary results. To achieve tumoricidal concentrations, current treatments such as melphalan and carboplatin are often administered in high doses that have severe side effects for the pediatric population including ischemia, neutropenia, a higher risk for development of a secondary malignancy, and blindness ${ }^{50,270,271}$. Previous work from our team identified how melphalan and carboplatin, two commonly administered chemotherapies to treat $\mathrm{Rb}$, increase retinal endothelial cell death and inflammation ${ }^{63,257}$. Even if high doses are 


\section{CD44}

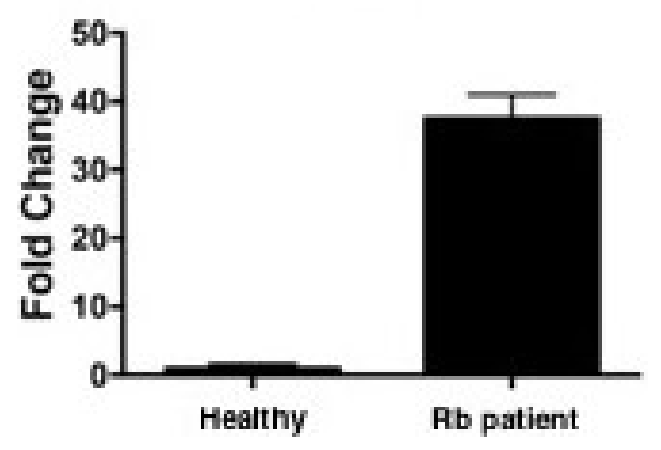

Figure 4-8. Expression of CD44 in naive human Rb vitreous.

qPCR analysis of mRNA isolated from ex vivo naïve $\mathrm{Rb}$ vitreous for $\mathrm{CD} 44$. $\mathrm{N}=5$ with 4 replicates per sample of human healthy vitreous. $\mathrm{N}=8$ with 4 replicates per sample of $\mathrm{Rb}$ vitreous. Bar graphs indicate results \pm SEM to control. 


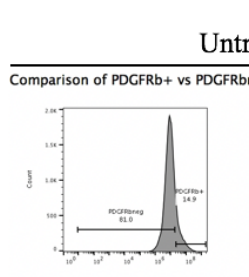

AFABB P.PDGFRB
Untreated

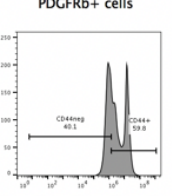

PercP-Cy5.5 Co 44

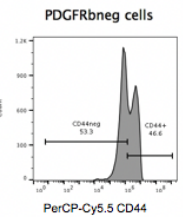

+ rhPDGF

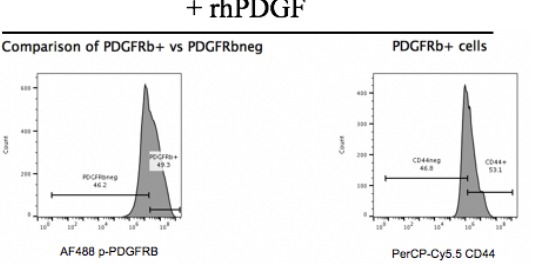

AF488 P.PDGFRB
PorCP.C5.5 CD24

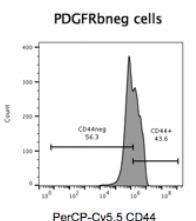

$+\mathrm{IM}$

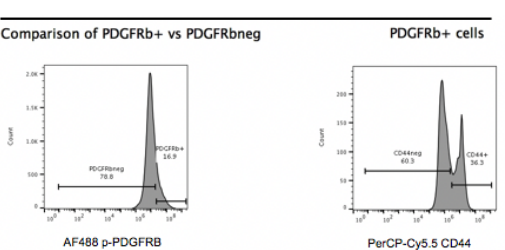

Perc-C.05.5 CDo44

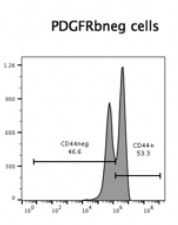

+ rhPDGF + IM

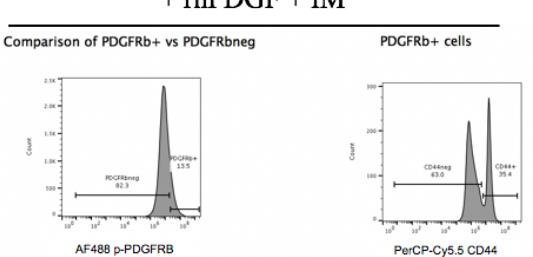

PDCFRbreg cells

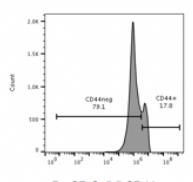

\section{Figure 4-9. CD44 is mediated by PDGFR $\beta$ activity.}

Assessment of CD44 positivity after disruption of PDGFR $\beta$ signaling. Y79 cells were treated for $24 \mathrm{~h}$ with $\mathrm{rhPDGF}$ (10ng/mL) or IM $(10 \mu \mathrm{M})$. After $24 \mathrm{~h}$, cells were collected and labelled with anti-human PerCP/Cy5.5 CD44 and p-PDGFR $\beta$ antibody with AlexaFluor ${ }^{\circledR}$ 488 secondary. Samples were run on a BioRad ZE5. Flow cytometry histogram is shown. N=3 with 3 replicates per sample. 
achieved, vitreous seeds are typically resistant to chemotherapy, further demonstrating the challenges in treating these seeds.

In this study, we first chose to focus on assessing potential cytotoxicity of antiPDGF-PDGFR $\beta$ therapy to endothelial cells. As endothelial cells are necessary to supply the healthy neural retina with nutrients via blood supply, significant loss of these endothelial cells can result in neurodegeneration and, ultimately, vision loss. These endothelial cells are also the primary component of the blood-retinal barrier (BRB) that prevents cellular infiltration into the healthy retina and use of an anti-PDGFR $\beta$ therapy could help maintain this essential structure ${ }^{272,273}$. Our study demonstrates the proapoptotic effects of IM are Rb cell specific, as hRECs proliferation and angiogenic activity are not affected. In contrast to human umbilical vein endothelial cells (hUVEC), which are widely used in in vitro studies of endothelial cells, hRECs do not signal through the PDGFR $\beta$. This was demonstrated in our studies by genomic and flow cytometry analyses. This work suggests anti-PDGFR $\beta$ therapy could be an Rb tumorspecific therapy.

This work suggests that disruption of this signaling pathway is a targeted and safe therapy. Next, we chose to also explore drug resistance. As mentioned above, vitreous seeds are challenging to treat not only due to their unique location but also because they are resistant to chemotherapy. Members of our collaborative team had previously identified members of the ATP-binding cassette (ABC) transporter family in $\mathrm{Rb}$ samples $^{266}$. Immunohistochemical analyses on naive ex vivo samples demonstrated the expression of MDR1, MRP1, and MRP2. BCRP was not detected in any of the cohort samples. In addition to this pioneering study, further work comparing naïve samples and samples obtained patients who had undergone systemic chemotherapy demonstrated this expression was not induced (or altered) by chemotherapy ${ }^{267}$. Instead, this resistance is an innate feature of $\mathrm{Rb}$ tumors. Using freshly isolated ex vivo vitreous samples from patients with vitreous seeds, we analyzed mRNA levels and measured heterogeneic expression of $A B C B 1$ (MDR1), $A B C C 1$ (MRP1), $A B C C 2$ (MRP2), and, surprisingly, $A B C G 2$ (BCRP), further demonstrating multiple levels of complexity of these tumors.

The defining-role of these MDRPs in chemoresistance is their ability to efflux intracellular chemotherapy drugs extracellularly. To test the ability of Rb cells to efflux, we utilized a novel efflux assay kit. A cell-permeant, fluorescent dye is used to measure this efflux. There is an inverse relationship between efflux and fluorescence. As a control, we used the MDA-MB-231 cell line of metastatic breast cancer cells that have been shown to lack efflux activity ${ }^{274}$. We postulated a small number of $\mathrm{Rb}$ cells may be promoting chemoresistance. This could explain the non-statistical significance in our results. Future studies could evaluate efflux using ex vivo samples and not cell lines. While we were performing these studies recent work from Nair et al. illustrated a low percentage $(<5 \%)$ of Y79 cells express MDRPs ${ }^{263}$. With such a small percentage of cells, quantitation of efflux activity will be drowned out by the large cohort of cells collected. Nonetheless, we do observe small differences among treatment groups and future studies must be conducted to elucidate how PDGF-PDGFR $\beta$ signaling may promote ABC transporter activity and be a potential target to decrease chemoresistance. Additional 
unidentified proteins might contribute to $\mathrm{Rb}$ chemoresistance. A previous study demonstrated an unidentified member of the $\mathrm{ABC}$ transporter family mediates $\mathrm{Y} 79 \mathrm{ABC}$ transporter efflux ${ }^{275}$.

To further evaluate mechanisms involved in chemoresistance, we investigated the expression of the hyaluronan receptor, or CD44, in ex vivo Rb naive vitreous samples. CD44 has been implicated in chemoresistance in a number of tumors and is known to assist in the regulation of MDRPs and ABC transporters ${ }^{276}$. When we compared them to healthy vitreous, we found a surprisingly high amount of CD44 mRNA in our Rb vitreous samples. These results support our hypothesis on the importance of the TME in $\mathrm{Rb}$ and how this TME may promote vitreous seed survival and resistance through CD44 while providing further evidence that chemoresistance is also an inherent tumor property of $\mathrm{Rb}$.

In contrast to the efflux studies of the $\mathrm{ABC}$ transporters, we saw major differences in the percentage of CD44-positivity based on treatment. First, we measured a higher percentage of $\mathrm{p}$-PDGFR $\beta^{+} \mathrm{CD} 44^{+}$cells upon rhPDGF stimulation compared to baseline levels. Therefore, we hypothesized disruption of the PDGF-PDGFR $\beta$ signaling pathway by IM would reduce this phenotype. In fact, IM reduced the percentage of $\mathrm{p}$ PDGFR $\beta^{+} \mathrm{CD} 44^{+}$cells while increasing the percentage of double-negative cells ( $\mathrm{p}$ PDGFR $\left.\beta^{\text {neg }} C D 44^{\text {neg }}\right)$. Ultimately, these results demonstrate that interactions between $R b$ cells and the microenvironment contribute to chemoresistance and warrants further investigations.

The present study investigated the role of the PDGF-PDGFR $\beta$ signaling pathway in hRECs as well as potential mechanisms for drug resistance. As a functional readout of disruption of the PDGF-PDGFR $\beta$ signaling pathway in hRECs, we used genomic (PDGFRB mRNA expression), flow cytometry (percentage of PDGFR $\beta^{+}$cells and VEGFA-producing cells), and angiogenic (tube formation assay) approaches to verify targeting PDGF-PDGFR $\beta$ signaling is not cytotoxic to hRECs. Follow up studies are needed to investigate endothelial cell leukostasis and toxicity in vivo. We hypothesize that anti-PDGF-PDGFR $\beta$ therapy will be an Rb-specific therapy without the toxicities associated with chemotherapy.

We also investigated the expression of $\mathrm{ABC}$ transporters in ex vivo naïve vitreous samples from $\mathrm{Rb}$ patients and in vitro by using a cell line to understand chemoresistance mechanisms. Moreover, we performed a comprehensive analysis on the percentage of PDGFR $\beta^{+}$cells showing positivity to CD44, a protein associated with drug resistance mechanisms. This work, for the first time, provides a potential mechanism by which vitreous seeds may utilize their microenvironment for their own survival and chemotherapy resistance.

Drug resistance in cancer is a complex and multifactorial process involving multiple pathways and mediators and merits more, in-depth studies. Resistant Rb cell lines should be developed and mRNA analysis should compare members of the ABC transporter family to chemo-sensitive cells. Similar to this study, functionality should 
also be tested using the same (or equivalent) dye-based assay. As the major limitations of this work are the in vitro approach and lack of in vivo studies as well as the results that are correlative, not specifically causative, future studies must use the in vivo approach using the same orthotopic xenograft rabbit model previously mentioned. These cells may also be engrafted in to the rabbit model to form vitreous seeds and will allow for a more comprehensive, in vivo study to elucidate mechanistically, how vitreous seeds are able to resist chemotherapy. 


\section{CHAPTER 5. DISCUSSION}

In their groundbreaking reviews, Doug Hanahan and Robert Weinberg established six primary mechanisms for tumor development and survival ${ }^{104}$. Yet, it is now understood that tumor cells do not act alone and that these hallmarks are not inherently tumor celldependent. Tumors are not one-dimensional, independent entities, instead they are composed of multiple cell types such as fibroblasts, endothelial cells, macrophages and monocytes, leukocytes, and pericytes that communicate within cells and their microenvironment through multiple proteins, receptors, and chemokines and cytokines. The crucial role of these interactions between tumors and stromal cells has broad implications for the hallmarks of cancer including metastasis, resistance, treatment options, and ultimately patient survival. These decisive communications have been vastly studied in malignancies such as breast ${ }^{277}$ and pancreactic ${ }^{278}$ cancers. However, there is a lack of such knowledge in $\mathrm{Rb}$ and, specifically, vitreous seeding. While very little is currently known regarding presence (or absence) of stromal cell types and composition of the $\mathrm{Rb} \mathrm{TME}$, it has been established that $\mathrm{Rb}$ cells exhibit unique properties allowing them to survive in the semi-solid, semi-liquid vitreous microenvironment ( $\mathrm{Rb}$ seeds). Furthermore, these seeds have reduced rates of proliferation and they are resistant to conventional chemotherapeutics ${ }^{100,266}$. Therefore, to target vitreous seeds, we need therapeutics aimed specifically at reducing vitreous seed survival signaling and reducing resistance. The focus of the work described herein is 1) to establish Rb-TME interactions that promote tumor survival and 2) demonstrate how investigating the TME can provide essential knowledge and emphasize the need to study not only the tumor, but the microenvironment that surrounds it.

The origins of vitreous seeding remain unknown. However, one key step in vitreous seed formation is the disruption (or invasion) of the vitreoretinal interface, namely the inner limiting membrane (ILM) which is primarily composed of type IV and VI collagens ${ }^{279}$. We identified members of the matrix metalloproteinase (MMP) family were expressed in both metastatic (Y79) and non-metastatic (Weri-1) Rb cell lines. These two MMPs are gelatinases that are responsible for degrading type IV collagen. We further identified pharmacological inhibition of these MMPs resulted in decreased Rb cell migration and invasion via transcriptional regulation of MMP-2 and -9. Surprisingly, we did see significant decreases in cell survival, as well. MMPs regulation primarily occurs through mRNA transcription, perhaps most notably by the E2F transcription factor family $^{280}$. We demonstrated targeting MMPs decreased E2F levels that resulted in reduced MMP mRNA. Most notably was the discovery of different intrinsic properties of these cells. Despite their clear involvement in angiogenesis, MMPs do not appear to play a major role in angiogenesis for Y79 as illustrated by the lack of changes in two key angiogenic molecules (VEGF and Ang-2) and may be more involved in metastasis (TGF$\beta$ ); meanwhile, we demonstrated Weri-1 angiogenic activity as well as metastasis may be partially regulated through MMP activity.

As previous clinical trials targeting MMPs failed due to lack of specificity for MMPs, we feel MMPIs are not strong candidates for clinical administration. One area 
that may be beneficial to investigate is the targeting of tissue inhibitors of metalloproteinases (TIMPs) that regulate the activities of MMPs. Prior studies have demonstrated the expression and function of TIMPs in metastatic Rb, but their therapeutic potential has yet to be tested ${ }^{190}$. By targeting these TIMPs, toxicities associated with the prior therapies could potentially be circumvented.

As the vitreous contains growth factors, cytokines, and chemokines, access to this supply of signaling molecules may assist in tumor survival. However, often times growth factors are sequestered in ECM and may only be considered bioavailable after ECM remodeling (via molecules such as the MMPs) ${ }^{112}$. One such family of molecules, the PDGF family, have already been implicated and targeted in vitreoretinal pathologies ${ }^{113,114}$ as well as oncogenesis ${ }^{157}$. We describe here the expression of active PDGFR $\beta$ and abundance of its primary ligand, PDGF-BB using ex vivo samples. Disruption of PDGFPDGFR $\beta$ signaling resulted in decreases in proliferation and invasion as well as increases in apoptotic activity. We provide mechanistic insight in how this signaling cascade drives survival through MDM2 and NFKB signaling.

Resistance is one of the major challenges in cancer treatments. The use of targeted therapies and immunotherapy were thought to undermine this problem. While initially responsible to targeted therapies, cancer cells are able to adapt and utilize similar effector pathways to confer survival ${ }^{281}$. As members of the same family of PDGFs, we postulate VEGF-VEGFR signaling may represent a potential compensatory mechanism for resistance in $\mathrm{Rb}$. We found inhibition of PDGF-PDGFR $\beta$ signaling did not result in increases in VEGF-A or VEGFR activity by demonstrating no change in VEGFA (or $V E G F R 2$ ) mRNA as well as a significant decrease in VEGFR2 protein activity. These results do not rule out development of another compensatory mechanism and future in vivo studies should incorporate these potential shortcomings Xenografts should be generated using tumor samples from patients with recurrent disease and inhibition of PDGF-PDGFR $\beta$ signaling should be tested therapeutically against these resistant tumors. Regular measurement of other major receptor tyrosine kinases may prove beneficial in identifying other potential therapeutic targets that underlie survival.

The PDGFR $\beta$ inhibitor chosen in these studies, imatinib, was developed and first used as an inhibitor for the Bcr-Abl fusion protein associated with chronic myelogenous leukemia (CML) as well as acute lymphoblastic leukemia (ALL) ${ }^{236}$. Other targets of imatinib include stem cell factor receptor (c-Kit) and PDGFRs (both PDGFR $\alpha$ and PDGFR $\beta$ ). To investigate if these cytotoxic effects were PDGFR $\beta$-specific as we hypothesized, we utilized siRNA technology and targeted the PDGFRB gene. The disruption of PDGFR $\beta$ dysregulated the downstream targets and pro-survival factors MDM2 and NFאB resulting in increased $\mathrm{Rb}$ cell apoptosis and decreasing cell survival. This confirmed the role of PDGFR $\beta$ in Rb cell survival. Our pre-clinical studies evaluated the safety of IM as a potential therapy on primary human retinal endothelial cells (hRECs). Results from our collaborators previously demonstrated both carboplatin and melphalan induced hREC toxicity as well inflammation ${ }^{63,257,282,283}$. We did not observe any IM-associated toxicities in hRECs indicating a potential Rb-specific effect. These results are quite promising preclinically and demonstrate that targeting the PDGF- 
PDGFR $\beta$ signaling pathway may prevent toxicities associated with therapies currently in use. In addition to finding inhibition of PDGF-PDGFR $\beta$ signaling may be $\mathrm{Rb}$-specific, we demonstrated the necessity of using site-specific cells is vital for preclinical testing. Prior studies have also examined endothelial cells within the context of $\mathrm{Rb}$ but have utilized hUVECs. We observed hRECs do not express PDGFRB mRNA whereas other studies have demonstrated hUVECs do express PDGFR $\beta$. Therefore, future preclinical studies examining endothelial-Rb cell interactions should utilize physiologicallyconsistent cells that would normally inhabit the Rb TME, such as hRECs.

The lack of in vitro and in vivo preclinical models has exacerbated the gap in knowledge of vitreous seeds. To date, the one in vivo animal model capable of developing vitreous seeds is the rabbit orthotopic xenograft ${ }^{92}$. This is an expensive model to generate and maintain. To circumvent this caveat, we utilized magnetic nanoparticles to generate three-dimensional spheroids that closely resemble vitreous seeds. This technology provides an in vitro platform that is quick and reproducible while providing a more physiologically relevant, three-dimensional model that better recapitulates in vivo and ex vivo pathology. This 3D system will allow researchers to test pre-clinically potential therapies in addition to provide a "small scale" high-throughput screening for drug discovery. This model can be extended further and utilized for co-culture systems that utilize tumor cells along other cell types in the TME to generate spheroids as we demonstrated by establishing co-cultures with hRECs. Furthermore, pre-labeling cell populations (such as with CFSE) allows for the examination of individual cell types by immunofluorescence and flow cytometry. This methodology will allow in-depth analysis of cellular interactions within the microenvironment. This technique will provide valuable insight into how current and future therapies alter tumor properties and how multiple cell types may respond to these drugs.

Vitreous seeds are understood to be resistant to chemotherapy. While this is in part due to the anatomical location of seeds and the reduced proliferation rates of seeds -as most chemotherapies target rapidly dividing cells, current research has failed to elucidate mechanistic insights into why or how these seeds are capable of surviving. The vitreous is a unique microenvironment and these seeds reside in an avascular system. As most chemotherapeutic routes of delivery depend on diffusion through the bloodstream, cells in an avascular system are exposed to much lower concentrations of these drugs and this concentration is less likely to be cytotoxic. Moreover, cells that are sensitive are killed while those resistant cells persist and give rise to recurrence. However, when concentrations are much higher (e.g. through IViC administration), higher rates of ocular salvage are still not achieved, demonstrating this effect may be related specifically to these cells (and thereby, their microenvironment). To attempt to explain chemoresistance, previous work from members of our team demonstrated heterogenous expression of proteins involved in drug resistance (MDRPs) ${ }^{266}$. Follow up work using naïve eyes as well as those that had undergone treatment found no differences in expression of MDRPs indicating that treatment does not induce drug resistance, resistant cells are selected ${ }^{267}$. We confirmed these results using ex vivo naïve $\mathrm{Rb}$ vitreous samples and found heterogenous mRNA expression of these MDRPs. We also characterized these MDRPs in vitro using the Y79 $\mathrm{Rb}$ cell line and could not detect either $A B C B 1$ and $A B C G 2 \mathrm{mRNA}$, 
emphasizing the heterogeneity of the tumors and the importance of the interactions between the tumors and their microenvironment. To further measure these MDRP efflux capacity, we deployed a commercially-available assay kit and demonstrated low Y79 efflux activity. It is worth noting small changes were seen between treatment groups, though the physiological relevance of these small changes is unknown. We hypothesized this may be attributable to a small percentage of cells with active ABC transporters, as had been previously demonstrated ${ }^{263}$. Instead, we focused our attention on another protein associated with chemotherapy resistance: CD44.

Recent reports demonstrated Y79 cells that were more chemoresistant to carboplatin expressed two biomarkers -- CD133 and CD44 ${ }^{263}$. These resistant cells' phenotype was CD44 ${ }^{\text {hi }}$ and, surprisingly, CD133 ${ }^{\text {lo } 263}$. In order to further examine how TME may alter properties of the tumor and elaborate on others' previous studies, we examined the expression of CD44 and how PDGF from the microenvironment may induce a more resistant phenotype. We measured CD44 mRNA at higher levels ( 40fold) in naïve vitreous from $\mathrm{Rb}$ patients versus the healthy vitreous controls.

Resistant Y79 cells measured increased expression of CD44 mRNA. We have previously demonstrated PDGF-PDGFR $\beta$ signaling regulates $\mathrm{NF} \kappa \mathrm{B}$ signaling. Therefore, we investigated how PDGF-PDGFR $\beta$ signaling may contribute to CD44 mRNA expression as $C D 44$ is a target gene of the transcription factor NFkB. Ultimately, we revealed a high percentage of $\mathrm{p}$-PDGFR $\beta$ positive $\left(\operatorname{PDGFR} \beta^{+}\right)$cells were simultaneously positive for CD44 $\left(\mathrm{CD}_{4} 4^{+}\right)$; in turn, when PDGF-PDGFR $\beta$ signaling is inhibited by IM, there is a decrease in percentage of double-positive $\left(\mathrm{PDGFR} \beta^{+} / \mathrm{CD} 44^{+}\right)$cells and an increase in double-negative (PDGFR $\beta^{\text {neg }} / \mathrm{CD} 44^{\text {neg }}$ ) cells. Taken together, these results implicate how PDGF-PDGFR $\beta$ signaling may promote a more resistant phenotype through CD44. More work needs to be conducted on the potential importance of CD44 within resistant and recurrent disease. Cell lines overexpressing CD44 should be generated to determine the role(s) CD44 plays in recurrence and resistant in addition to in vitro and in vivo testing of anti-CD44 targeted therapies (such as previously conducted in ovarian cancer ${ }^{284}$ ) to examine potential therapeutic value.

This work has provided compelling evidence of the importance of the microenvironment in $\mathrm{Rb}$ growth, survival, response to treatment (or lack thereof), and metastasis. However, there is a still a lack of understanding about the vitreous as a tumor microenvironment in $\mathrm{Rb}$. As future advancements in genomic and proteomics continue, the vitreous microenvironment needs to be characterized. Future pre-clinical animal model studies will be essential to determine the therapeutic and clinical value antiPDGFR $\beta$ as a targeted therapy or sensitizing agent for $\mathrm{Rb}$. We foresee these studies should be conducted in vivo using the rabbit orthotopic xenograft as it is the only model capable of forming vitreous seeds. This model is also capable of withstanding multiple injections and more closely resembles the volume of a pediatric eye. Using this model, the role the $\mathrm{ABC}$ transporter family and CD44 may play in chemoresistance can also be determined. Preclinical examination targeting the PDGF-PDGFR $\beta$ signaling cascade could further demonstrate a link between microenvironment and promoting chemoresistance. In depth studies could further examine tumor features through use of 
human-specific antibodies and primers while comparing results to the host by employing rabbit-specific reagents. Our studies are groundwork for future studies to identify novel targets for therapy to clear vitreous seeds and raise ocular salvage rates much higher than the $70 \%$ that has been the norm for so long.

In conclusion, this work reveals, for the first time, how the TME may alter properties of $\mathrm{Rb}$ cells. We demonstrated how interactions with the ECM by the MMPs promotes invasion and migration and that, when these interactions are inhibited, $\mathrm{Rb}$ cell secretions are altered and survival decreases. We identified highly active PDGFR $\beta$ within the vitreous of ex vivo human $\mathrm{Rb}$ tissue and discovered the roles the PDGF-PDGFR $\beta$ signaling pathway plays in $\mathrm{Rb}$ cell proliferation, invasion, and survival. Disruption of the PDGF-PDGFR $\beta$ signaling pathway resulted in reduced proliferation and invasion, as well as increase in percentage of apoptotic cells. We provided mechanistic insight that illustrated this survival signaling may be mediated by MDM2 and NFkB. Furthermore, as demonstrated targeting PDGFR $\beta$ does not induce toxicities in hRECs and this therapeutic target may be Rb-cell specific. We examined the role the PDGFR $\beta$ signaling cascade may play in promoting resistance to chemotherapy. We indicated this drug resistance is an inherent property of these $\mathrm{Rb}$ cells and denoted heterogeneity of the $\mathrm{ABC}$ transporters. We found CD44 highly expressed in the vitreous of naïve human $\mathrm{Rb}$ vitreous and demonstrated how cells with active PDGFR $\beta$ are also positive for CD44 and, when this signaling is disrupted, CD44 concomitantly decreases. Ultimately, targeting the PDGFPDGFR $\beta$ signaling pathway may be useful in targeting chemoresistant cells. Based on our findings, we developed a working model (Figure 5-1). 

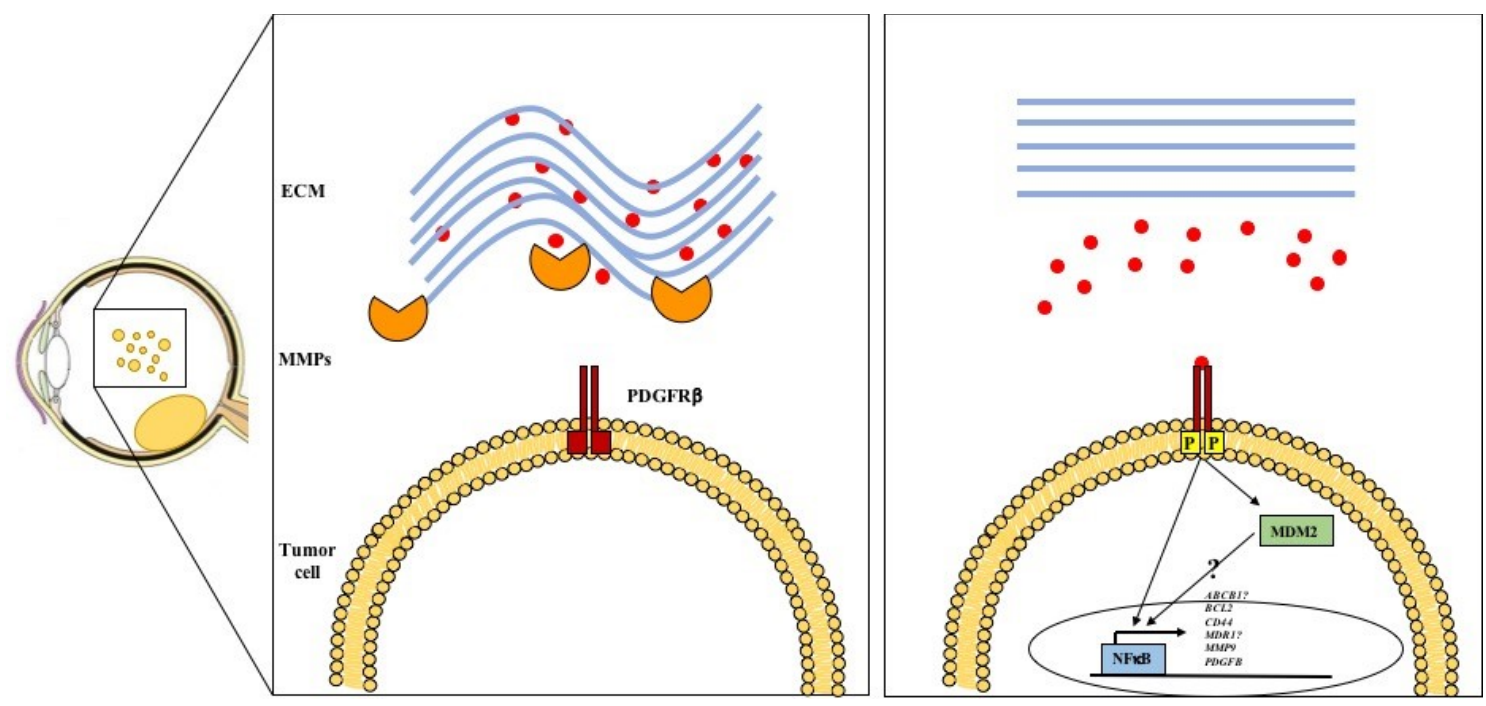

Figure 5-1. Working model of vitreous seed microenvironment.

$\mathrm{Rb}$ cells express MMPs. Upon entrance in to the vitreous, these cells secrete these gelatinases (orange) that induce ECM degradation and remodeling (blue). This remodeling frees sequestered growth factors (i.e. PDGF, red) that are, in turn, free to bind receptors. Ligand-receptor interactions induce a downstream signaling cascade that results in anti-apoptotic activity via MDM2 as well as pro-survival (and pro-resistance) signaling through $\mathrm{NF} \kappa \mathrm{B}$. Ultimately, access to the vitreous confers on these cells as growth and survival advantage that is difficult to overcome. . 


\section{LIST OF REFERENCES}

1. Chung EM, Specht CS, Schroeder JW. From the archives of the AFIP: Pediatric orbit tumors and tumorlike lesions: neuroepithelial lesions of the ocular globe and optic nerve. Radiographics. 2007;27(4):1159-1186.

2. Kivelä T, Polkunen M. Pieter Pauw's tumor oculorum: Reappraisal of the presumed first description of retinoblastoma in 1597. Archives of Ophthalmology. 2003;121(6):881-886.

3. Abramson DH. Retinoblastoma in the 20th Century: Past Success and Future Challenges The Weisenfeld Lecture. Investigative Ophthalmology \& Visual Science. 2005;46(8):2684-2691.

4. Pandey AN. Retinoblastoma: An overview. Saudi Journal of Ophthalmology. 2014;28(4):310-315.

5. Grossniklaus HE. Retinoblastoma. Fifty Years of Progress. The LXXI Edward Jackson Memorial Lecture. American journal of ophthalmology. 2014;158(5):875-891.e871.

6. Reese AB, Ellsworth RM. The evaluation and current concept of retinoblastoma therapy. Trans Am Acad Ophthalmol Otolaryngol. 1963;67:164-172.

7. Ellsworth RM. The practical management of retinoblastoma. Transactions of the American Ophthalmological Society. 1969;67:462-534.

8. Shields CL, Mashayekhi A, Au AK, et al. The International Classification of Retinoblastoma predicts chemoreduction success. Ophthalmology. 2006;113(12):2276-2280.

9. Linn Murphree A. Intraocular retinoblastoma: the case for a new group classification. Ophthalmol Clin North Am. 2005;18(1):41-53, viii.

10. Knudson AG. Mutation and Cancer: Statistical Study of Retinoblastoma. Proceedings of the National Academy of Sciences. 1971;68(4):820.

11. Lalande M, Dryja TP, Schreck RR, Shipley J, Flint A, Latt SA. Isolation of human chromosome 13-specific DNA sequences cloned from flow sorted chromosomes and potentially linked to the retinoblastoma locus. Cancer genetics and cytogenetics. 1984;13(4):283-295.

12. Friend SH, Bernards R, Rogelj S, et al. A human DNA segment with properties of the gene that predisposes to retinoblastoma and osteosarcoma. Nature. 1986;323(6089):643-646.

13. Goodrich DW, Wang NP, Qian YW, Lee EY, Lee WH. The retinoblastoma gene product regulates progression through the G1 phase of the cell cycle. Cell. 1991;67(2):293-302.

14. Rigberg DA, Kim FS, Sebastian JL, Kazanjian KK, McFadden DW. Hypophosphorylated retinoblastoma protein is associated with G2 arrest in esophageal squamous cell carcinoma. The Journal of surgical research. 1999;84(1):101-105.

15. Knudsen ES, Wang JY. Dual mechanisms for the inhibition of E2F binding to RB by cyclin-dependent kinase-mediated RB phosphorylation. Molecular and cellular biology. 1997;17(10):5771-5783. 
16. Narasimha AM, Kaulich M, Shapiro GS, Choi YJ, Sicinski P, Dowdy SF. Cyclin $\mathrm{D}$ activates the $\mathrm{Rb}$ tumor suppressor by mono-phosphorylation. eLife. 2014;3:e02872.

17. Blais A, van Oevelen CJ, Margueron R, Acosta-Alvear D, Dynlacht BD. Retinoblastoma tumor suppressor protein-dependent methylation of histone $\mathrm{H} 3$ lysine 27 is associated with irreversible cell cycle exit. The Journal of cell biology. 2007;179(7):1399-1412.

18. Wistuba, II, Gazdar AF, Minna JD. Molecular genetics of small cell lung carcinoma. Seminars in oncology. 2001;28(2 Suppl 4):3-13.

19. Bäcklund LM, Nilsson BR, Liu L, Ichimura K, Collins VP. Mutations in Rb1 pathway-related genes are associated with poor prognosis in Anaplastic Astrocytomas. British Journal Of Cancer. 2005;93:124.

20. Witkiewicz AK, Knudsen ES. Retinoblastoma tumor suppressor pathway in breast cancer: prognosis, precision medicine, and therapeutic interventions. Breast Cancer Research : BCR. 2014;16(3):207-207.

21. Rushlow D, Piovesan B, Zhang K, et al. Detection of mosaic RB1 mutations in families with retinoblastoma. Human mutation. 2009;30(5):842-851.

22. Rushlow DE, Mol BM, Kennett JY, et al. Characterisation of retinoblastomas without RB1 mutations: genomic, gene expression, and clinical studies. The Lancet Oncology. 2013;14(4):327-334.

23. Dimaras H, Khetan V, Halliday W, et al. Loss of RB1 induces non-proliferative retinoma: increasing genomic instability correlates with progression to retinoblastoma. Human Molecular Genetics. 2008;17(10):1363-1372.

24. Laurie NA, Donovan SL, Shih C-S, et al. Inactivation of the p53 pathway in retinoblastoma. Nature. 2006;444:61.

25. Guo Y, Pajovic S, Gallie BL. Expression of p14ARF, MDM2, and MDM4 in human retinoblastoma. Biochem Biophys Res Commun. 2008;375(1):1-5.

26. Kooi IE, Mol BM, Massink MPG, et al. Somatic genomic alterations in retinoblastoma beyond RB1 are rare and limited to copy number changes. Scientific Reports. 2016;6:25264.

27. Zhang J, Benavente CA, McEvoy J, et al. A Novel Retinoblastoma Therapy from Genomic and Epigenetic Analyses. Nature. 2012;481(7381):329-334.

28. Dimaras H, Corson TW, Cobrinik D, et al. Retinoblastoma. Nature reviews Disease primers. 2015;1:15021-15021.

29. Usmanov RH, Kivela T. Predicted Trends in the Incidence of Retinoblastoma in the Asia-Pacific Region. Asia Pac J Ophthalmol (Phila). 2014;3(3):151-157.

30. Butros LJ, Abramson DH, Dunkel IJ. Delayed Diagnosis of Retinoblastoma: Analysis of Degree, Cause, and Potential Consequences. Pediatrics. 2002;109(3):e45.

31. Aerts I, Lumbroso-Le Rouic L, Gauthier-Villars M, Brisse H, Doz F, Desjardins L. Retinoblastoma. Orphanet Journal of Rare Diseases. 2006;1:31-31.

32. Canturk S, Qaddoumi I, Khetan V, et al. Survival of retinoblastoma in lessdeveloped countries impact of socioeconomic and health-related indicators. $\mathrm{Br} J$ Ophthalmol. 2010;94(11):1432-1436.

33. Naseripour M. "Retinoblastoma survival disparity": The expanding horizon in developing countries. Saudi Journal of Ophthalmology. 2012;26(2):157-161. 
34. Ibrahim N, Maysa A-H, Aamer A, et al. The Impact of Growth Patterns of Retinoblastoma (Endophytic, Exophytic, and Mixed Patterns) / Retinoblastomda Büyüme Paternlerinin Etkisi (Endofitik, Ekzofitik ve Karışık Paternler). Turkish Journal of Pathology. 2015;31(1):45-50.

35. Chong E-M, Coffee RE, Chintagumpala M, Hurwitz RL, Hurwitz MY, ChévezBarrios P. Extensively Necrotic Retinoblastoma Is Associated With High-Risk Prognostic Factors. Archives of Pathology \& Laboratory Medicine. 2006;130(11):1669-1672.

36. Traine PG, Schedler KJ, Rodrigues EB. Clinical Presentation and Genetic Paradigm of Diffuse Infiltrating Retinoblastoma: A Review. Ocular Oncology and Pathology. 2016;2(3):128-132.

37. Chévez-Barrios P, Hurwitz MY, Louie K, et al. Metastatic and Nonmetastatic Models of Retinoblastoma. The American Journal of Pathology. 2000;157(4):1405-1412.

38. Nakao S, Hafezi-Moghadam A, Ishibashi T. Lymphatics and Lymphangiogenesis in the Eye. Journal of Ophthalmology. 2012;2012:783163.

39. Chen X, Wang J, Cao Z, et al. Invasiveness and metastasis of retinoblastoma in an orthotopic zebrafish tumor model. Scientific Reports. 2015;5:10351.

40. Gündüz K, Müftüoglu O, Günalp İ, Ünal E, Taçyıllız N. Metastatic Retinoblastoma: Clinical Features, Treatment, and Prognosis. Ophthalmology. 2006;113(9):1558-1566.

41. Shields JA, Parsons H, Shields CL, Giblin ME. The role of cryotherapy in the management of retinoblastoma. Am J Ophthalmol. 1989;108(3):260-264.

42. Chintagumpala M, Chevez-Barrios P, Paysse EA, Plon SE, Hurwitz R. Retinoblastoma: Review of Current Management. The Oncologist. 2007;12(10):1237-1246.

43. Shields CL, Santos MM, Diniz W, et al. Thermotherapy for retinoblastoma. Archives of Ophthalmology. 1999;117(7):885-893.

44. Hamel P, Heon E, Gallie BL, Budning AS. Focal therapy in the management of retinoblastoma: When to start and when to stop. Journal of American Association for Pediatric Ophthalmology and Strabismus. 2000;4(6):334-337.

45. Abramson DH, Jereb B, Ellsworth RM. External beam radiation for retinoblastoma. Bulletin of the New York Academy of Medicine. 1981;57(9):787803.

46. Kim J-Y, Park Y. Treatment of Retinoblastoma: The Role of External Beam Radiotherapy. Yonsei Medical Journal. 2015;56(6):1478-1491.

47. Abramson DH, Beaverson KL, Chang ST, Dunkel IJ, McCormick B. Outcome following initial external beam radiotherapy in patients withreese-ellsworth group vb retinoblastoma. Archives of Ophthalmology. 2004;122(9):1316-1323.

48. Marees T, Moll AC, Imhof SM, de Boer MR, Ringens PJ, van Leeuwen FE. Risk of Second Malignancies in Survivors of Retinoblastoma: More Than 40 Years of Follow-up. JNCI: Journal of the National Cancer Institute. 2008;100(24):17711779.

49. Wong F, Boice JD, Jr, Abramson DH, et al. Cancer incidence after retinoblastoma: Radiation dose and sarcoma risk. JAMA. 1997;278(15):12621267. 
50. Wong JR, Morton LM, Tucker MA, et al. Risk of Subsequent Malignant Neoplasms in Long-Term Hereditary Retinoblastoma Survivors After Chemotherapy and Radiotherapy. Journal of Clinical Oncology. 2014;32(29):3284-3290.

51. Mouw KW, Sethi RV, Yeap BY, et al. Proton Radiotherapy for the Treatment of Retinoblastoma. International journal of radiation oncology, biology, physics. 2014;90(4):863-869.

52. Merchant TE, Gould CJ, Wilson MW, Hilton NE, Rodriguez-Galindo C, Haik BG. Episcleral plaque brachytherapy for retinoblastoma. Pediatric Blood \& Cancer. 2004;43(2):134-139.

53. Gombos DS, Kelly A, Coen PG, Kingston JE, Hungerford JL. Retinoblastoma treated with primary chemotherapy alone: the significance of tumour size, location, and age. Br J Ophthalmol. 2002;86(1):80-83.

54. Varan A, Kiratli H, Aydin B, et al. The treatment of retinoblastoma with fourdrug regimen including cisplatin, etoposide, vincristine, and cyclophosphamide. Pediatric hematology and oncology. 2012;29(6):529-537.

55. Shahsavari M, Mashayekhi A. Pharmacotherapy for Retinoblastoma. Journal of Ophthalmic \& Vision Research. 2009;4(3):169-173.

56. Dasari S, Tchounwou PB. Cisplatin in cancer therapy: molecular mechanisms of action. European journal of pharmacology. 2014;0:364-378.

57. Gidding CEM, Kellie SJ, Kamps WA, de Graaf SSN. Vincristine revisited. Critical Reviews in Oncology / Hematology. 1999;29(3):267-287.

58. Montecucco A, Zanetta F, Biamonti G. Molecular mechanisms of etoposide. EXCLI Journal. 2015;14:95-108.

59. Lawley PD, Phillips DH. DNA adducts from chemotherapeutic agents. Mutation research. 1996;355(1-2):13-40.

60. Gallie BL, Budning A, DeBoer G, et al. Chemotherapy with focal therapy can cure intraocular retinoblastoma without radiotherapy. Arch Ophthalmol. 1996;114(11):1321-1328.

61. Turaka K, Shields CL, Meadows AT, Leahey A. Second malignant neoplasms following chemoreduction with carboplatin, etoposide, and vincristine in 245 patients with intraocular retinoblastoma. Pediatr Blood Cancer. 2012;59(1):121125.

62. Abramson DH, Dunkel IJ, Brodie SE, Kim JW, Gobin YP. A phase I/II study of direct intraarterial (ophthalmic artery) chemotherapy with melphalan for intraocular retinoblastoma initial results. Ophthalmology. 2008;115(8):1398-1404, 1404.e1391.

63. Tse BC, Steinle JJ, Johnson D, Haik BG, Wilson MW. Superselective intraophthalmic artery chemotherapy in a nonhuman primate model: histopathologic findings. JAMA Ophthalmol. 2013;131(7):903-911.

64. Abramson DH, Daniels AB, Marr BP, et al. Intra-Arterial Chemotherapy (Ophthalmic Artery Chemosurgery) for Group D Retinoblastoma. PLoS One. 2016;11(1):e0146582.

65. Daniels AB, Froehler MT, Pierce JM, et al. Pharmacokinetics, Tissue Localization, Toxicity, and Treatment Efficacy in the First Small Animal (Rabbit) 
Model of Intra-Arterial Chemotherapy for Retinoblastoma. Investigative Ophthalmology \& Visual Science. 2018;59(1):446-454.

66. Hayden BH, Murray TG, Scott IU, et al. Subconjunctival carboplatin in retinoblastoma: Impact of tumor burden and dose schedule. Archives of Ophthalmology. 2000;118(11):1549-1554.

67. Brennan RC, Qaddoumi I, Mao S, et al. Ocular Salvage and Vision Preservation Using a Topotecan-Based Regimen for Advanced Intraocular Retinoblastoma. Journal of Clinical Oncology. 2017;35(1):72-77.

68. Ghassemi F, Shields CL. Intravitreal melphalan for refractory or recurrent vitreous seeding from retinoblastoma. Archives of Ophthalmology. 2012;130(10):1268-1271.

69. Francis JH, Marr BP, Brodie SE, Abramson DH. Anterior Ocular Toxicity of Intravitreous Melphalan for Retinoblastoma. JAMA ophthalmology. 2015;133(12):1459-1463.

70. Bosaleh A, Sampor C, Solernou V, et al. Outcome of children with retinoblastoma and isolated choroidal invasion. Archives of Ophthalmology. 2012;130(6):724729.

71. Zhang L, Gao T, Shen Y. Quality of life in children with retinoblastoma after enucleation in China. Pediatric Blood \& Cancer. 2018;65(7):e27024.

72. Sultan I, Wilson MW, Nawaiseh I, et al. Enucleation for retinoblastoma: the experience of a single center in Jordan. Int Ophthalmol. 2010;30(4):407-414.

73. Jaradat I, Mubiden R, Salem A, Abdel-Rahman F, Al-Ahmad I, Almousa A. High-dose chemotherapy followed by stem cell transplantation in the management of retinoblastoma: a systematic review. Hematology/oncology and stem cell therapy. 2012;5(2):107-117.

74. Dunkel IJ, Krailo MD, Chantada GL, et al. Intensive multi-modality therapy for extra-ocular retinoblastoma (RB): A Children's Oncology Group (COG) trial (ARET0321). Journal of Clinical Oncology. 2017;35(15_suppl):10506-10506.

75. Kremens B, Wieland R, Reinhard H, et al. High-dose chemotherapy with autologous stem cell rescue in children with retinoblastoma. Bone Marrow Transplantation. 2003;31:281.

76. Rodriguez-Galindo C, Wilson MW, Haik BG, et al. Treatment of metastatic retinoblastoma. Ophthalmology. 2003;110(6):1237-1240.

77. Caselli D, Tamburini A, La Torre A, et al. High-dose chemotherapy with autologous stem cell rescue for treatment of retinoblastoma: Report of five cases. Pediatric Transplantation. 2014;18(6):631-636.

78. Dunkel IJ, Aledo A, Kernan NA, et al. Successful treatment of metastatic retinoblastoma. Cancer. 2000;89(10):2117-2121.

79. Reid TW, Albert DM, Rabson AS, et al. Characteristics of an Established Cell Line of Retinoblastoma2. JNCI: Journal of the National Cancer Institute. 1974;53(2):347-360.

80. McFall RC, Sery TW, Makadon M. Characterization of a new continuous cell line derived from a human retinoblastoma. Cancer Res. 1977;37(4):1003-1010.

81. Fournier GA, Sang DN, Albert DM, Craft JL. Electron microscopy and HLA expression of a new cell line of retinoblastoma. Investigative Ophthalmology \& Visual Science. 1987;28(4):690-699. 
82. Madreperla SA, Bookstein R, Jones OW, Lee WH. Retinoblastoma cell lines Y79, $\mathrm{RB} 355$ and WERI-Rb27 are genetically related. Ophthalmic paediatrics and genetics. 1991;12(1):49-56.

83. O'Brien JM, Marcus DM, Niffenegger AS, et al. Trilateral retinoblastoma in transgenic mice. Transactions of the American Ophthalmological Society. 1989;87:301-322; discussion 322-306.

84. Hamel PA, Cohen BL, Sorce LM, Gallie BL, Phillips RA. Hyperphosphorylation of the retinoblastoma gene product is determined by domains outside the simian virus 40 large-T-antigen-binding regions. Molecular and cellular biology. 1990;10(12):6586-6595.

85. Robanus-Maandag E, Dekker M, van der Valk M, et al. p107 is a suppressor of retinoblastoma development in $\mathrm{pRb}$-deficient mice. Genes \& development. 1998;12(11):1599-1609.

86. MacPherson D. Insights from mouse models into human retinoblastoma. Cell Division. 2008;3:9-9.

87. Nair RM, Kaliki S, Vemuganti GK. Animal models in retinoblastoma research. Saudi Journal of Ophthalmology. 2013;27(3):141-146.

88. McEvoy J, Flores-Otero J, Zhang J, et al. Coexpression of Normally Incompatible Developmental Pathways in Retinoblastoma Genesis. Cancer cell. 2011;20(2):260-275.

89. Howard MA, Wardwell S, Albert DM. Effect of butyrate and corticosteroids on retinoblastoma in vitro and in vivo. Investigative Ophthalmology \& Visual Science. 1991;32(6):1711-1713.

90. Laurie NA, Gray JK, Zhang J, et al. Topotecan combination chemotherapy in two new rodent models of retinoblastoma. Clinical cancer research : an official journal of the American Association for Cancer Research. 2005;11(20):75697578.

91. Cassoux N, Thuleau A, Assayag F, Aerts I, Decaudin D. Establishment of an Orthotopic Xenograft Mice Model of Retinoblastoma Suitable for Preclinical Testing. Ocular Oncology and Pathology. 2015;1(3):200-206.

92. Kang SJ, Grossniklaus HE. Rabbit Model of Retinoblastoma. Journal of Biomedicine and Biotechnology. 2011;2011:5.

93. Aerts I, Leuraud P, Blais J, et al. In vivo efficacy of photodynamic therapy in three new xenograft models of human retinoblastoma. Photodiagnosis and photodynamic therapy. 2010;7(4):275-283.

94. Shields CL, Honavar SG, Meadows AT, et al. Chemoreduction plus focal therapy for retinoblastoma: factors predictive of need for treatment with external beam radiotherapy or enucleation. Am J Ophthalmol. 2002;133(5):657-664.

95. Pascual-Pasto G, Olaciregui NG, Opezzo JAW, et al. Increased delivery of chemotherapy to the vitreous by inhibition of the blood-retinal barrier. Journal of controlled release : official journal of the Controlled Release Society. 2017;264:34-44.

96. Abramson DH, Marr BP, Dunkel IJ, et al. Intra-arterial chemotherapy for retinoblastoma in eyes with vitreous and/or subretinal seeding: 2-year results. $\mathrm{Br} J$ Ophthalmol. 2012;96(4):499-502. 
97. Suzuki S, Yamane T, Mohri M, Kaneko A. Selective ophthalmic arterial injection therapy for intraocular retinoblastoma: the long-term prognosis. Ophthalmology. 2011;118(10):2081-2087.

98. Munier FL, Gaillard MC, Balmer A, et al. Intravitreal chemotherapy for vitreous disease in retinoblastoma revisited: from prohibition to conditional indications. $\mathrm{Br}$ J Ophthalmol. 2012;96(8):1078-1083.

99. Manjandavida FP, Shields CL. The role of intravitreal chemotherapy for retinoblastoma. Indian Journal of Ophthalmology. 2015;63(2):141-145.

100. Munier FL. Classification and Management of Seeds in RetinoblastomaEllsworth Lecture Ghent August 24th 2013. Ophthalmic Genetics. 2014;35(4):193-207.

101. Amram AL, Rico G, Kim JW, et al. Vitreous Seeds in Retinoblastoma: Clinicopathologic Classification and Correlation. Ophthalmology. 2017;124(10):1540-1547.

102. Dalgard CL, Van Quill KR, O'Brien JM. Evaluation of the in vitro and in vivo antitumor activity of histone deacetylase inhibitors for the therapy of retinoblastoma. Clinical cancer research : an official journal of the American Association for Cancer Research. 2008;14(10):3113-3123.

103. Paget S. THE DISTRIBUTION OF SECONDARY GROWTHS IN CANCER OF THE BREAST. The Lancet. 1889;133(3421):571-573.

104. Hanahan D, Weinberg RA. Hallmarks of cancer: the next generation. Cell. 2011;144(5):646-674.

105. Piña Y, Boutrid H, Murray TG, et al. Impact of Tumor-Associated Macrophages in LH(BETA)T(AG) Mice on Retinal Tumor Progression: Relation to Macrophage Subtype. Investigative Ophthalmology \& Visual Science. 2010;51(5):2671-2677.

106. Boutrid H, Jockovich M-E, Murray TG, et al. Targeting Hypoxia, a Novel Treatment for Advanced Retinoblastoma. Investigative Ophthalmology \& Visual Science. 2008;49(7):2799-2805.

107. Cosse JP, Michiels C. Tumour hypoxia affects the responsiveness of cancer cells to chemotherapy and promotes cancer progression. Anti-cancer agents in medicinal chemistry. 2008;8(7):790-797.

108. Dawson DW, Volpert OV, Gillis P, et al. Pigment Epithelium-Derived Factor: A Potent Inhibitor of Angiogenesis. Science. 1999;285(5425):245.

109. Dai Y, Wu Z, Sheng H, Zhang Z, Yu M, Zhang Q. Identification of inflammatory mediators in patients with rhegmatogenous retinal detachment associated with choroidal detachment. Mol Vis. 2015;21:417-427.

110. Monteiro JP, Santos FM, Rocha AS, et al. Vitreous humor in the pathologic scope: insights from proteomic approaches. Proteomics Clinical applications. 2015;9(1-2):187-202.

111. Pollreisz A, Funk M, Breitwieser FP, et al. Quantitative proteomics of aqueous and vitreous fluid from patients with idiopathic epiretinal membranes. Exp Eye Res. 2013;108:48-58.

112. Schultz GS, Wysocki A. Interactions between extracellular matrix and growth factors in wound healing. Wound repair and regeneration: official publication of the Wound Healing Society [and] the European Tissue Repair Society. 2009;17(2):153-162. 
113. Morescalchi F, Duse S, Gambicorti E, Romano MR, Costagliola C, Semeraro F. Proliferative Vitreoretinopathy after Eye Injuries: An Overexpression of Growth Factors and Cytokines Leading to a Retinal Keloid. Mediators of Inflammation. 2013;2013:12.

114. Angi M, Kalirai H, Coupland SE, Damato BE, Semeraro F, Romano MR. Proteomic Analyses of the Vitreous Humour. Mediators of Inflammation. 2012;2012:7.

115. Frantz C, Stewart KM, Weaver VM. The extracellular matrix at a glance. Journal of Cell Science. 2010;123(24):4195-4200.

116. Giannandrea M, Parks WC. Diverse functions of matrix metalloproteinases during fibrosis. Disease Models \& Mechanisms. 2014;7(2):193-203.

117. Plantner JJ, Smine A, Quinn TA. Matrix metalloproteinases and metalloproteinase inhibitors in human interphotoreceptor matrix and vitreous. Curr Eye Res. 1998;17(2):132-140.

118. Lan J, Kumar RK, Di Girolamo N, McCluskey P, Wakefield D. Expression and distribution of matrix metalloproteinases and their inhibitors in the human iris and ciliary body. The British Journal of Ophthalmology. 2003;87(2):208-211.

119. Caley MP, Martins VLC, O'Toole EA. Metalloproteinases and Wound Healing. Advances in Wound Care. 2015;4(4):225-234.

120. Ljubimov AV, Saghizadeh M. Progress in corneal wound healing. Progress in retinal and eye research. 2015;49:17-45.

121. Kowluru RA, Zhong Q, Santos JM. Matrix metalloproteinases in diabetic retinopathy: potential role of MMP-9. Expert opinion on investigational drugs. 2012;21(6):797-805.

122. Rundhaug JE. Matrix metalloproteinases and angiogenesis. Journal of cellular and molecular medicine. 2005;9(2):267-285.

123. De Groef L, Van Hove I, Dekeyster E, Stalmans I, Moons L. MMPs in the trabecular meshwork: promising targets for future glaucoma therapies? Invest Ophthalmol Vis Sci. 2013;54(12):7756-7763.

124. De La Paz MA, Itoh Y, Toth CA, Nagase H. Matrix metalloproteinases and their inhibitors in human vitreous. Invest Ophthalmol Vis Sci. 1998;39(7):1256-1260.

125. Noel A, Jost M, Maquoi E. Matrix metalloproteinases at cancer tumor-host interface. Seminars in cell \& developmental biology. 2008;19(1):52-60.

126. Cathcart J, Pulkoski-Gross A, Cao J. Targeting matrix metalloproteinases in cancer: Bringing new life to old ideas. Genes \& Diseases. 2015;2(1):26-34.

127. Vihinen P, Kähäri V-M. Matrix metalloproteinases in cancer: Prognostic markers and therapeutic targets. International Journal of Cancer. 2002;99(2):157-166.

128. Hadler-Olsen E, Winberg JO, Uhlin-Hansen L. Matrix metalloproteinases in cancer: their value as diagnostic and prognostic markers and therapeutic targets. Tumour Biol. 2013;34(4):2041-2051.

129. Seyfried TN, Huysentruyt LC. On the Origin of Cancer Metastasis. Critical reviews in oncogenesis. 2013;18(1-2):43-73.

130. Lamouille S, Xu J, Derynck R. Molecular mechanisms of epithelial-mesenchymal transition. Nature reviews Molecular cell biology. 2014;15(3):178-196. 
131. Fischer KR, Durrans A, Lee S, et al. Epithelial-to-mesenchymal transition is not required for lung metastasis but contributes to chemoresistance. Nature. 2015;527:472.

132. Wojtowicz-Praga S, Low J, Marshall J, et al. Phase I trial of a novel matrix metalloproteinase inhibitor batimastat (BB-94) in patients with advanced cancer. Investigational New Drugs. 1996;14(2):193-202.

133. Overall CM, Kleifeld O. Towards third generation matrix metalloproteinase inhibitors for cancer therapy. British Journal of Cancer. 2006;94(7):941-946.

134. Watson SA, Morris TM, Collins HM, Bawden LJ, Hawkins K, Bone EA. Inhibition of tumour growth by marimastat in a human xenograft model of gastric cancer: relationship with levels of circulating CEA. Br J Cancer. 1999;81(1):1923.

135. Bramhall SR, Hallissey MT, Whiting J, et al. Marimastat as maintenance therapy for patients with advanced gastric cancer: a randomised trial. British Journal of Cancer. 2002;86(12):1864-1870.

136. Andrae J, Gallini R, Betsholtz C. Role of platelet-derived growth factors in physiology and medicine. Genes \& development. 2008;22(10):1276-1312.

137. Van Stry M, Kazlauskas A, Schreiber SL, Symes K. Distinct effectors of plateletderived growth factor receptor- $\alpha$ signaling are required for cell survival during embryogenesis. Proceedings of the National Academy of Sciences of the United States of America. 2005;102(23):8233-8238.

138. Boström H, Willetts K, Pekny M, et al. PDGF-A Signaling Is a Critical Event in Lung Alveolar Myofibroblast Development and Alveogenesis. Cell. 1996;85(6):863-873.

139. Leveen P, Pekny M, Gebre-Medhin S, Swolin B, Larsson E, Betsholtz C. Mice deficient for PDGF B show renal, cardiovascular, and hematological abnormalities. Genes \& development. 1994;8(16):1875-1887.

140. Ding H, Wu X, Bostrom H, et al. A specific requirement for PDGF-C in palate formation and PDGFR-alpha signaling. Nature genetics. 2004;36(10):1111-1116.

141. Fredriksson L, Nilsson I, Su EJ, et al. Platelet-Derived Growth Factor C Deficiency in C57BL/6 Mice Leads to Abnormal Cerebral Vascularization, Loss of Neuroependymal Integrity, and Ventricular Abnormalities. The American Journal of Pathology. 2012;180(3):1136-1144.

142. Gladh H, Folestad EB, Muhl L, et al. Mice Lacking Platelet-Derived Growth Factor D Display a Mild Vascular Phenotype. PLOS ONE. 2016;11(3):e0152276.

143. Hoch RV, Soriano P. Roles of PDGF in animal development. Development. 2003;130(20):4769.

144. Fruttiger M, Calver AR, Kruger WH, et al. PDGF mediates a neuron-astrocyte interaction in the developing retina. Neuron. 1996;17(6):1117-1131.

145. Mudhar HS, Pollock RA, Wang C, Stiles CD, Richardson WD. PDGF and its receptors in the developing rodent retina and optic nerve. Development. 1993;118(2):539-552.

146. Klinghoffer RA, Mueting-Nelsen PF, Faerman A, Shani M, Soriano P. The Two PDGF Receptors Maintain Conserved Signaling In Vivo despite Divergent Embryological Functions. Molecular Cell. 2001;7(2):343-354. 
147. Seo MS, Okamoto N, Vinores MA, et al. Photoreceptor-specific expression of platelet-derived growth factor-B results in traction retinal detachment. Am J Pathol. 2000;157(3):995-1005.

148. Mori K, Gehlbach P, Ando A, et al. Retina-Specific Expression of PDGF-B Versus PDGF-A: Vascular Versus Nonvascular Proliferative Retinopathy. Investigative Ophthalmology \& Visual Science. 2002;43(6):2001-2006.

149. Freyberger H, Brocker M, Yakut H, et al. Increased levels of platelet-derived growth factor in vitreous fluid of patients with proliferative diabetic retinopathy. Experimental and clinical endocrinology \& diabetes : official journal, German Society of Endocrinology [and] German Diabetes Association. 2000;108(2):106109.

150. Inaba T, Ishibashi S, Gotoda T, et al. Enhanced expression of platelet-derived growth factor-beta receptor by high glucose. Involvement of platelet-derived growth factor in diabetic angiopathy. Diabetes. 1996;45(4):507-512.

151. Praidou A, Klangas I, Papakonstantinou E, et al. Vitreous and serum levels of platelet-derived growth factor and their correlation in patients with proliferative diabetic retinopathy. Curr Eye Res. 2009;34(2):152-161.

152. Praidou A, Papakonstantinou E, Androudi S, Georgiadis N, Karakiulakis G, Dimitrakos S. Vitreous and serum levels of vascular endothelial growth factor and platelet-derived growth factor and their correlation in patients with nonproliferative diabetic retinopathy and clinically significant macula oedema. Acta Ophthalmologica. 2011;89(3):248-254.

153. Jo N, Mailhos C, Ju M, et al. Inhibition of Platelet-Derived Growth Factor B Signaling Enhances the Efficacy of Anti-Vascular Endothelial Growth Factor Therapy in Multiple Models of Ocular Neovascularization. The American Journal of Pathology. 2006;168(6):2036-2053.

154. Akiyama H, Kachi S, Silva RL, et al. Intraocular injection of an aptamer that binds PDGF-B: a potential treatment for proliferative retinopathies. Journal of cellular physiology. 2006;207(2):407-412.

155. Dunn EN, Hariprasad SM, Sheth VS. An Overview of the Fovista and Rinucumab Trials and the Fate of Anti-PDGF Medications. Ophthalmic Surg Lasers Imaging Retina. 2017;48(2):100-104.

156. Folkman J. Seminars in Medicine of the Beth Israel Hospital, Boston. Clinical applications of research on angiogenesis. The New England journal of medicine. 1995;333(26):1757-1763.

157. Heldin $\mathrm{CH}$, Westermark B. Mechanism of action and in vivo role of plateletderived growth factor. Physiological reviews. 1999;79(4):1283-1316.

158. Uhrbom L, Hesselager G, Nister M, Westermark B. Induction of brain tumors in mice using a recombinant platelet-derived growth factor B-chain retrovirus. Cancer Res. 1998;58(23):5275-5279.

159. Koschmann C, Zamler D, MacKay A, et al. Characterizing and targeting PDGFRA alterations in pediatric high-grade glioma. Oncotarget. 2016;7(40):65696-65706.

160. Lau D, Magill ST, Aghi MK. Molecularly targeted therapies for recurrent glioblastoma: current and future targets. Neurosurgical Focus. 2014;37(6):E15. 
161. Balachandran VP, DeMatteo RP. GIST tumors: Who should get imatinib and for how long? Advances in surgery. 2014;48(1):165-183.

162. Takahira T, Oda Y, Tamiya S, et al. Detection of COL1A1-PDGFB fusion transcripts and PDGFB/PDGFRB mRNA expression in dermatofibrosarcoma protuberans. Modern Pathology. 2007;20:668.

163. Noujaim J, Thway K, Fisher C, Jones RL. Dermatofibrosarcoma protuberans: from translocation to targeted therapy. Cancer Biology \& Medicine. 2015;12(4):375-384.

164. Bernatchez PN, Soker S, Sirois MG. Vascular Endothelial Growth Factor Effect on Endothelial Cell Proliferation, Migration, and Platelet-activating Factor Synthesis Is Flk-1-dependent. Journal of Biological Chemistry. 1999;274(43):31047-31054.

165. Falk AT, Barriere J, Francois E, Follana P. Bevacizumab: A dose review. Critical reviews in oncology/hematology. 2015;94(3):311-322.

166. Lee SY, Kim DK, Cho JH, Koh JY, Yoon YH. Inhibitory effect of bevacizumab on the angiogenesis and growth of retinoblastoma. Arch Ophthalmol. 2008;126(7):953-958.

167. Assayag F, Nicolas A, Vacher S, et al. Combination of Carboplatin and Bevacizumab Is an Efficient Therapeutic Approach in Retinoblastoma PatientDerived Xenografts. Investigative Ophthalmology \& Visual Science. 2016;57(11):4916-4926.

168. Kim LA, D'Amore PA. A Brief History of Anti-VEGF for the Treatment of Ocular Angiogenesis. The American Journal of Pathology. 2012;181(2):376-379.

169. Rofagha S, Bhisitkul RB, Boyer DS, Sadda SR, Zhang K. Seven-year outcomes in ranibizumab-treated patients in ANCHOR, MARINA, and HORIZON: a multicenter cohort study (SEVEN-UP). Ophthalmology. 2013;120(11):22922299.

170. Webb AH, Gao BT, Goldsmith ZK, et al. Inhibition of MMP-2 and MMP-9 decreases cellular migration, and angiogenesis in in vitro models of retinoblastoma. BMC Cancer. 2017;17(1):434.

171. Broaddus E, Topham A, Singh AD. Incidence of retinoblastoma in the USA: 1975-2004. Br J Ophthalmol. 2009;93(1):21-23.

172. Lohmann DR, Gallie BL. Retinoblastoma: revisiting the model prototype of inherited cancer. Am J Med Genet C Semin Med Genet. 2004;129C(1):23-28.

173. Abramson DH, Ellsworth RM, Grumbach N, Kitchin FD. Retinoblastoma: survival, age at detection and comparison 1914-1958, 1958-1983. J Pediatr Ophthalmol Strabismus. 1985;22(6):246-250.

174. Abramson DH, Ellsworth RM, Grumbach N, Sturgis-Buckhout L, Haik BG. Retinoblastoma: correlation between age at diagnosis and survival. J Pediatr Ophthalmol Strabismus. 1986;23(4):174-177.

175. Erwenne CM, Franco EL. Age and lateness of referral as determinants of extraocular retinoblastoma. Ophthalmic Paediatr Genet. 1989;10(3):179-184.

176. Rubenfeld M, Abramson DH, Ellsworth RM, Kitchin FD. Unilateral vs. bilateral retinoblastoma. Correlations between age at diagnosis and stage of ocular disease. Ophthalmology. 1986;93(8):1016-1019. 
177. Bader JL, Meadows AT, Zimmerman LE, et al. Bilateral retinoblastoma with ectopic intracranial retinoblastoma: trilateral retinoblastoma. Cancer Genet Cytogenet. 1982;5(3):203-213.

178. Messmer EP, Heinrich T, Hopping W, de Sutter E, Havers W, Sauerwein W. Risk factors for metastases in patients with retinoblastoma. Ophthalmology. 1991;98(2):136-141.

179. Shields CL, Shields JA, Baez KA, Cater J, De Potter PV. Choroidal invasion of retinoblastoma: metastatic potential and clinical risk factors. Br J Ophthalmol. 1993;77(9):544-548.

180. Sastre X, Chantada GL, Doz F, et al. Proceedings of the consensus meetings from the International Retinoblastoma Staging Working Group on the pathology guidelines for the examination of enucleated eyes and evaluation of prognostic risk factors in retinoblastoma. Arch Pathol Lab Med. 2009;133(8):1199-1202.

181. Klein T, Bischoff R. Physiology and pathophysiology of matrix metalloproteases. Amino Acids. 2011;41(2):271-290.

182. Overall CM. Molecular determinants of metalloproteinase substrate specificity: matrix metalloproteinase substrate binding domains, modules, and exosites. $\mathrm{Mol}$ Biotechnol. 2002;22(1):51-86.

183. Page-McCaw A, Ewald AJ, Werb Z. Matrix metalloproteinases and the regulation of tissue remodelling. Nat Rev Mol Cell Biol. 2007;8(3):221-233.

184. Foda HD, Zucker S. Matrix metalloproteinases in cancer invasion, metastasis and angiogenesis. Drug Discov Today. 2001;6(9):478-482.

185. Wieczorek E, Jablonska E, Wasowicz W, Reszka E. Matrix metalloproteinases and genetic mouse models in cancer research: a mini-review. Tumour Biol. 2015;36(1):163-175.

186. Devy L, Dransfield DT. New Strategies for the Next Generation of MatrixMetalloproteinase Inhibitors: Selectively Targeting Membrane-Anchored MMPs with Therapeutic Antibodies. Biochem Res Int. 2011;2011:191670.

187. Zucker S, Hymowitz M, Rollo EE, et al. Tumorigenic potential of extracellular matrix metalloproteinase inducer. Am J Pathol. 2001;158(6):1921-1928.

188. GileadSciences. Safety and Tolerability Study in Solid Tumors. https:/clinicaltrials.gov/ct2/show/NCT01803282?term=NCT01803282. Accessed 05.26.2016.

189. Marshall DC, Lyman SK, McCauley S, et al. Selective Allosteric Inhibition of MMP9 Is Efficacious in Preclinical Models of Ulcerative Colitis and Colorectal Cancer. PloS one. 2015;10(5):e0127063.

190. Adithi M, Nalini V, Kandalam M, Krishnakumar S. Expression of matrix metalloproteinases and their inhibitors in retinoblastoma. Journal of pediatric hematology/oncology. 2007;29(6):399-405.

191. Long H, Zhou B, Jiang FG. Expression of MMP-2 and MMP-9 in retinoblastoma and their significance. Int $J$ Ophthalmol. 2011;4(5):489-491.

192. Chintalapudi SR, Djenderedjian L, Stiemke AB, Steinle JJ, Jablonski MM, Morales-Tirado VM. Isolation and Molecular Profiling of Primary Mouse Retinal Ganglion Cells: Comparison of Phenotypes from Healthy and Glaucomatous Retinas. Front Aging Neurosci. 2016;8:93. 
193. Chintalapudi SR, Morales-Tirado VM, Williams RW, Jablonski MM.

Multipronged approach to identify and validate a novel upstream regulator of Sncg in mouse retinal ganglion cells. FEBS J. 2016;283(4):678-693.

194. Morales-Tirado V, Johannson S, Hanson E, et al. Cutting edge: selective requirement for the Wiskott-Aldrich syndrome protein in cytokine, but not chemokine, secretion by CD4+ T cells. J Immunol. 2004;173(2):726-730.

195. Gao BT, Lee RP, Jiang Y, Steinle JJ, Morales-Tirado VM. Pioglitazone alters monocyhttps://clinicaltrials.gov/ct2/show/NCT01803282?term=NCT01803282.te populations and stimulates recent thymic emigrants in the BBDZR/Wor type 2 diabetes rat model. Diabetol Metab Syndr. 2015;7:72.

196. Thakran S, Zhang Q, Morales-Tirado V, Steinle JJ. Pioglitazone restores IGFBP-3 levels through DNA PK in retinal endothelial cells cultured in hyperglycemic conditions. Invest Ophthalmol Vis Sci. 2015;56(1):177-184.

197. Zhang Q, Jiang Y, Toutounchian J, et al. Novel quinic acid derivative KZ-41 prevents retinal endothelial cell apoptosis without inhibiting retinoblastoma cell death through p38 signaling. Invest Ophthalmol Vis Sci. 2013;54(9):5937-5943.

198. Vandenbroucke RE, Libert C. Is there new hope for therapeutic matrix metalloproteinase inhibition? Nat Rev Drug Discov. 2014;13(12):904-927.

199. Gialeli C, Theocharis AD, Karamanos NK. Roles of matrix metalloproteinases in cancer progression and their pharmacological targeting. The FEBS Journal. 2011;278(1):16-27.

200. Yan C, Boyd DD. Regulation of matrix metalloproteinase gene expression. Journal of cellular physiology. 2007;211(1):19-26.

201. Ren B, Cam H, Takahashi Y, et al. E2F integrates cell cycle progression with DNA repair, replication, and $\mathrm{G}(2) / \mathrm{M}$ checkpoints. Genes \& development. 2002;16(2):245-256.

202. Albert DM, Tapper D, Robinson NL, Felman R. Retinoblastoma and angiogenesis activity. Retina. 1984;4(3):189-194.

203. Hollborn M, Stathopoulos C, Steffen A, Wiedemann P, Kohen L, Bringmann A. Positive feedback regulation between MMP-9 and VEGF in human RPE cells. Invest Ophthalmol Vis Sci. 2007;48(9):4360-4367.

204. Zhu Y, Lee C, Shen F, Du R, Young WL, Yang GY. Angiopoietin-2 facilitates vascular endothelial growth factor-induced angiogenesis in the mature mouse brain. Stroke. 2005;36(7):1533-1537.

205. Holash J, Maisonpierre PC, Compton D, et al. Vessel cooption, regression, and growth in tumors mediated by angiopoietins and VEGF. Science. 1999;284(5422):1994-1998.

206. Ferrari G, Cook BD, Terushkin V, Pintucci G, Mignatti P. Transforming growth factor-beta 1 (TGF-beta1) induces angiogenesis through vascular endothelial growth factor (VEGF)-mediated apoptosis. Journal of cellular physiology. 2009;219(2):449-458.

207. Krstic J, Santibanez JF. Transforming growth factor-beta and matrix metalloproteinases: functional interactions in tumor stroma-infiltrating myeloid cells. ScientificWorldJournal. 2014;2014:521754.

208. Guo P, Imanishi Y, Cackowski FC, et al. Up-regulation of angiopoietin-2, matrix metalloprotease-2, membrane type 1 metalloprotease, and laminin 5 gamma 2 
correlates with the invasiveness of human glioma. Am J Pathol. 2005;166(3):877890.

209. Carmeliet P. Angiogenesis in health and disease. Nat Med. 2003;9(6):653-660.

210. Derynck R, Zhang YE. Smad-dependent and Smad-independent pathways in TGF-beta family signalling. Nature. 2003;425(6958):577-584.

211. Miettinen PJ, Ebner R, Lopez AR, Derynck R. TGF-beta induced transdifferentiation of mammary epithelial cells to mesenchymal cells: involvement of type I receptors. J Cell Biol. 1994;127(6 Pt 2):2021-2036.

212. Piek E, Moustakas A, Kurisaki A, Heldin CH, ten Dijke P. TGF-(beta) type I receptor/ALK-5 and Smad proteins mediate epithelial to mesenchymal transdifferentiation in NMuMG breast epithelial cells. J Cell Sci. 1999;112 ( Pt 24):4557-4568.

213. Kim ES, Kim MS, Moon A. TGF-beta-induced upregulation of MMP-2 and MMP-9 depends on p38 MAPK, but not ERK signaling in MCF10A human breast epithelial cells. Int J Oncol. 2004;25(5):1375-1382.

214. Oshimori N, Oristian D, Fuchs E. TGF-beta promotes heterogeneity and drug resistance in squamous cell carcinoma. Cell. 2015;160(5):963-976.

215. Crosby MB, Hubbard GB, Gallie BL, Grossniklaus HE. Anterior diffuse retinoblastoma: mutational analysis and immunofluorescence staining. Arch Pathol Lab Med. 2009;133(8):1215-1218.

216. Goldsmith ZK, Coppess W, Irvine AS, et al. Targeting the Platelet-Derived Growth Factor-beta Stimulatory Circuitry to Control Retinoblastoma Seeds. Investigative Ophthalmology \& Visual Science. 2018;59(11):4486-4495.

217. Shields CL, Honavar SG, Shields JA, Demirci H, Meadows AT, Naduvilath TJ. Factors predictive of recurrence of retinal tumors, vitreous seeds, and subretinal seeds following chemoreduction for retinoblastoma. Arch Ophthalmol. 2002;120(4):460-464.

218. Gunduz K, Gunalp I, Yalcindag N, et al. Causes of chemoreduction failure in retinoblastoma and analysis of associated factors leading to eventual treatment with external beam radiotherapy and enucleation. Ophthalmology. 2004;111(10):1917-1924.

219. Kaneko A, Suzuki S. Eye-preservation treatment of retinoblastoma with vitreous seeding. Jpn J Clin Oncol. 2003;33(12):601-607.

220. Munier FL, Soliman S, Moulin AP, Gaillard MC, Balmer A, Beck-Popovic M. Profiling safety of intravitreal injections for retinoblastoma using an anti-reflux procedure and sterilisation of the needle track. Br J Ophthalmol. 2012;96(8):1084-1087.

221. Shields CL, Douglass AM, Beggache M, Say EA, Shields JA. INTRAVITREOUS CHEMOTHERAPY FOR ACTIVE VITREOUS SEEDING FROM RETINOBLASTOMA: Outcomes After 192 Consecutive Injections. The 2015 Howard Naquin Lecture. Retina. 2016;36(6):1184-1190.

222. Chantada GL, Sampor C, Bosaleh A, Solernou V, Fandino A, de Davila MT. Comparison of staging systems for extraocular retinoblastoma: analysis of 533 patients. JAMA Ophthalmol. 2013;131(9):1127-1134. 
223. Aman J, van Bezu J, Damanafshan A, et al. Effective treatment of edema and endothelial barrier dysfunction with imatinib. Circulation. 2012;126(23):27282738.

224. Manjandavida FP, Honavar SG, Reddy VA, Khanna R. Management and outcome of retinoblastoma with vitreous seeds. Ophthalmology. 2014;121(2):517-524.

225. Angi M, Kalirai H, Coupland SE, Damato BE, Semeraro F, Romano MR. Proteomic analyses of the vitreous humour. Mediators Inflamm. 2012;2012:148039.

226. Morescalchi F, Duse S, Gambicorti E, Romano MR, Costagliola C, Semeraro F. Proliferative vitreoretinopathy after eye injuries: an overexpression of growth factors and cytokines leading to a retinal keloid. Mediators Inflamm. 2013;2013:269787.

227. Ni Z, Hui P. Emerging pharmacologic therapies for wet age-related macular degeneration. Ophthalmologica. 2009;223(6):401-410.

228. McAuley AK, Sanfilippo PG, Hewitt AW, et al. Vitreous biomarkers in diabetic retinopathy: a systematic review and meta-analysis. J Diabetes Complications. 2014;28(3):419-425.

229. Dai C, Celestino JC, Okada Y, Louis DN, Fuller GN, Holland EC. PDGF autocrine stimulation dedifferentiates cultured astrocytes and induces oligodendrogliomas and oligoastrocytomas from neural progenitors and astrocytes in vivo. Genes Dev. 2001;15(15):1913-1925.

230. Dong J, Grunstein J, Tejada M, et al. VEGF-null cells require PDGFR alpha signaling-mediated stromal fibroblast recruitment for tumorigenesis. $E M B O J$. 2004;23(14):2800-2810.

231. Shih AH, Dai C, Hu X, Rosenblum MK, Koutcher JA, Holland EC. Dosedependent effects of platelet-derived growth factor-B on glial tumorigenesis. Cancer Res. 2004;64(14):4783-4789.

232. Guo $\mathrm{P}, \mathrm{Hu} \mathrm{B}, \mathrm{Gu} \mathrm{W}$, et al. Platelet-derived growth factor-B enhances glioma angiogenesis by stimulating vascular endothelial growth factor expression in tumor endothelia and by promoting pericyte recruitment. Am J Pathol. 2003;162(4):1083-1093.

233. Heldin CH. Targeting the PDGF signaling pathway in tumor treatment. Cell Commun Signal. 2013;11:97.

234. Xue Y, Lim S, Yang Y, et al. PDGF-BB modulates hematopoiesis and tumor angiogenesis by inducing erythropoietin production in stromal cells. Nat Med. 2011;18(1):100-110.

235. Yamane K, Minamoto A, Yamashita H, et al. Proteome analysis of human vitreous proteins. Molecular \& cellular proteomics : MCP. 2003;2(11):11771187.

236. Druker BJ, Sawyers CL, Kantariian H, et al. Activity of a specific inhibitor of the BCR-ABL tyrosine kinase in the blast crisis of chronic myeloid leukemia and acute lymphoblastic leukemia with the Philadelphia chromosome. $N$ Engl J Med. 2001;344(14):1038-1042.

237. Druker BJ, Talpaz M, Resta DJ, et al. Efficacy and safety of a specific inhibitor of the BCR-ABL tyrosine kinase in chronic myeloid leukemia. $N$ Engl J Med. 2001;344(14):1031-1037. 
238. Kvanta A, Steen B, Seregard S. Expression of vascular endothelial growth factor (VEGF) in retinoblastoma but not in posterior uveal melanoma. Exp Eye Res. 1996;63(5):511-518.

239. Arean C, Orellana ME, Abourbih D, Abreu C, Pifano I, Burnier MN, Jr. Expression of vascular endothelial growth factor in retinoblastoma. Arch Ophthalmol. 2010;128(2):223-229.

240. Sanft DM, Worme MD, Rielo de Moura L, et al. Immunohistochemical Analysis of PDGFR-alpha, PDGFR-beta and c-Abl in Retinoblastoma: Potential Therapeutic Targets. Ophthalmic Res. 2016;55(3):159-162.

241. de Moura LR, Marshall JC, Di Cesare S, Fernandes BF, Antecka E, Burnier MN. The effect of imatinib mesylate on the proliferation, invasive ability, and radiosensitivity of retinoblastoma cell lines. Eye (Lond). 2013;27(1):92-99.

242. Buchdunger E, Zimmermann J, Mett H, et al. Inhibition of the Abl proteintyrosine kinase in vitro and in vivo by a 2-phenylaminopyrimidine derivative. Cancer Res. 1996;56(1):100-104.

243. Carroll M, Ohno-Jones S, Tamura S, et al. CGP 57148, a tyrosine kinase inhibitor, inhibits the growth of cells expressing BCR-ABL, TEL-ABL, and TELPDGFR fusion proteins. Blood. 1997;90(12):4947-4952.

244. Sawyers CL, Hochhaus A, Feldman E, et al. Imatinib induces hematologic and cytogenetic responses in patients with chronic myelogenous leukemia in myeloid blast crisis: results of a phase II study. Blood. 2002;99(10):3530-3539.

245. Talpaz M, Silver RT, Druker BJ, et al. Imatinib induces durable hematologic and cytogenetic responses in patients with accelerated phase chronic myeloid leukemia: results of a phase 2 study. Blood. 2002;99(6):1928-1937.

246. Kantarjian H, Sawyers C, Hochhaus A, et al. Hematologic and cytogenetic responses to imatinib mesylate in chronic myelogenous leukemia. $N$ Engl J Med. 2002;346(9):645-652.

247. Merchant MS, Woo CW, Mackall CL, Thiele CJ. Potential use of imatinib in Ewing's Sarcoma: evidence for in vitro and in vivo activity. J Natl Cancer Inst. 2002;94(22):1673-1679.

248. Cortes J, Giles F, O'Brien S, et al. Results of imatinib mesylate therapy in patients with refractory or recurrent acute myeloid leukemia, high-risk myelodysplastic syndrome, and myeloproliferative disorders. Cancer. 2003;97(11):2760-2766.

249. Demetri GD, von Mehren M, Blanke CD, et al. Efficacy and safety of imatinib mesylate in advanced gastrointestinal stromal tumors. $N$ Engl $\mathrm{J} \mathrm{Med.}$ 2002;347(7):472-480.

250. Hassler MR, Vedadinejad M, Flechl B, et al. Response to imatinib as a function of target kinase expression in recurrent glioblastoma. Springerplus. 2014;3:111.

251. Adzemovic MV, Zeitelhofer M, Eriksson U, Olsson T, Nilsson I. Imatinib ameliorates neuroinflammation in a rat model of multiple sclerosis by enhancing blood-brain barrier integrity and by modulating the peripheral immune response. PloS one. 2013;8(2):e56586.

252. Crespo O, Kang SC, Daneman R, et al. Tyrosine kinase inhibitors ameliorate autoimmune encephalomyelitis in a mouse model of multiple sclerosis. $J$ Clin Immunol. 2011;31(6):1010-1020. 
253. Xin GH, Zhao XH, Liu D, et al. Effect of VEGF-targeted antisense gene therapy on retinoblastoma cell line SO-RB50 in vitro and in vivo. Int J Ophthalmol. 2012;5(4):440-447.

254. Heo JW, Kim JH, Cho CS, et al. Inhibitory activity of bevacizumab to differentiation of retinoblastoma cells. PloS one. 2012;7(3):e33456.

255. Haisler WL, Timm DM, Gage JA, Tseng H, Killian TC, Souza GR. Threedimensional cell culturing by magnetic levitation. Nat Protoc. 2013;8(10):19401949.

256. Amram AL, Rico G, Kim JW, et al. Vitreous Seeds in Retinoblastoma: Clinicopathologic Classification and Correlation. Ophthalmology. 2017.

257. Steinle JJ, Zhang Q, Thompson KE, et al. Intra-ophthalmic artery chemotherapy triggers vascular toxicity through endothelial cell inflammation and leukostasis. Invest Ophthalmol Vis Sci. 2012;53(4):2439-2445.

258. Zhang Q, Cheng Y, Huang L, Bai Y, Liang J, Li X. Inhibitory effect of carboplatin in combination with bevacizumab on human retinoblastoma in an in vitro and in vivo model. Oncology letters. 2017;14(5):5326-5332.

259. Luqmani YA. Mechanisms of drug resistance in cancer chemotherapy. Medical principles and practice : international journal of the Kuwait University, Health Science Centre. 2005;14 Suppl 1:35-48.

260. Senthebane DA, Rowe A, Thomford NE, et al. The Role of Tumor Microenvironment in Chemoresistance: To Survive, Keep Your Enemies Closer. International Journal of Molecular Sciences. 2017;18(7):1586.

261. Binder MJ, McCoombe S, Williams ED, McCulloch DR, Ward AC. The extracellular matrix in cancer progression: Role of hyalectan proteoglycans and ADAMTS enzymes. Cancer letters. 2017;385:55-64.

262. Shukla SJ, Duan S, Wu X, Badner JA, Kasza K, Dolan ME. Whole-genome approach implicates CD44 in cellular resistance to carboplatin. Human Genomics. 2009;3(2):128-142.

263. Nair RM, Balla MMS, Khan I, Kalathur RKR, Kondaiah P, Vemuganti GK. In vitro characterization of CD133(lo) cancer stem cells in Retinoblastoma Y79 cell line. BMC Cancer. 2017;17:779.

264. Bourguignon LYW, Peyrollier K, Xia W, Gilad E. Hyaluronan-CD44 interaction activates stem cell marker Nanog, Stat-3-mediated MDR1 gene expression, and ankyrin-regulated multidrug efflux in breast and ovarian tumor cells. The Journal of biological chemistry. 2008;283(25):17635-17651.

265. Cailleau R, Young R, Olivé M, Reeves JWJ. Breast Tumor Cell Lines From Pleural Effusions2. JNCI: Journal of the National Cancer Institute. 1974;53(3):661-674.

266. Wilson MW, Fraga CH, Fuller CE, et al. Immunohistochemical detection of multidrug-resistant protein expression in retinoblastoma treated by primary enucleation. Invest Ophthalmol Vis Sci. 2006;47(4):1269-1273.

267. Wilson MW, Fraga CH, Rodriguez-Galindo C, Hagedorn N, Leggas ML, Stewart C. Expression of the Multi-Drug Resistance Proteins and the Pregnane X Receptor in Treated and Untreated Retinoblastoma. Current Eye Research. 2009;34(5):386-394. 
268. Pietras K, Stumm M, Hubert M, et al. STI571 enhances the therapeutic index of epothilone B by a tumor-selective increase of drug uptake. Clinical cancer research : an official journal of the American Association for Cancer Research. 2003;9(10 Pt 1):3779-3787.

269. Abramson DH, Dunkel IJ, Brodie SE, Marr B, Gobin YP. Superselective ophthalmic artery chemotherapy as primary treatment for retinoblastoma (chemosurgery). Ophthalmology. 2010;117(8):1623-1629.

270. Schmack I, Hubbard GB, Kang SJ, Aaberg TM, Jr., Grossniklaus HE. Ischemic Necrosis and Atrophy of the Optic Nerve After Periocular Carboplatin Injection for Intraocular Retinoblastoma. American Journal of Ophthalmology. 2006;142(2):310-315.e312.

271. Gobin YP, Dunkel IJ, Marr BP, Brodie SE, Abramson DH. Intra-arterial chemotherapy for the management of retinoblastoma: four-year experience. Arch Ophthalmol. 2011;129(6):732-737.

272. Crane IJ, Liversidge J. Mechanisms of leukocyte migration across the bloodretina barrier. Seminars in Immunopathology. 2008;30(2):165-177.

273. Xu H, Manivannan A, Goatman KA, et al. Reduction in shear stress, activation of the endothelium, and leukocyte priming are all required for leukocyte passage across the blood--retina barrier. Journal of leukocyte biology. 2004;75(2):224232.

274. Bao L, Hazari S, Mehra S, Kaushal D, Moroz K, Dash S. Increased Expression of P-Glycoprotein and Doxorubicin Chemoresistance of Metastatic Breast Cancer Is Regulated by miR-298. The American Journal of Pathology. 2012;180(6):24902503.

275. Ishikawa Y, Nagai J, Okada Y, Sato K, Yumoto R, Takano M. Function and expression of ATP-binding cassette transporters in cultured human Y79 retinoblastoma cells. Biological \& pharmaceutical bulletin. 2010;33(3):504-511.

276. Toole BP, Slomiany MG. Hyaluronan, CD44 and Emmprin: partners in cancer cell chemoresistance. Drug resistance updates : reviews and commentaries in antimicrobial and anticancer chemotherapy. 2008;11(3):110-121.

277. Place AE, Jin Huh S, Polyak K. The microenvironment in breast cancer progression: biology and implications for treatment. Breast Cancer Research. 2011;13(6):227.

278. Whatcott CJ, Hanl H, Von Hoff DD. Orchestrating the Tumor Microenvironment to Improve Survival for Patients With Pancreatic Cancer Normalization, Not Destruction. Cancer journal (Sudbury, Mass). 2015;21(4):299-306.

279. Ponsioen TL, van Luyn MJA, van der Worp RJ, van Meurs JC, Hooymans JMM, Los LI. Collagen Distribution in the Human Vitreoretinal Interface. Investigative Ophthalmology \& Visual Science. 2008;49(9):4089-4095.

280. Johnson JL, Pillai S, Pernazza D, Sebti SM, Lawrence NJ, Chellappan SP. Regulation of matrix metalloproteinase genes by E2F transcription factors: RbRaf-1 interaction as a novel target for metastatic disease. Cancer Res. 2012;72(2):516-526.

281. Logue JS, Morrison DK. Complexity in the signaling network: insights from the use of targeted inhibitors in cancer therapy. Genes \& development. 2012;26(7):641-650. 
282. Ditta LC, Choudhri AF, Tse BC, et al. Validating a nonhuman primate model of super-selective intraophthalmic artery chemotherapy: comparing ophthalmic artery diameters. Invest Ophthalmol Vis Sci. 2012;53(12):7791-7794.

283. Wilson MW, Jackson JS, Phillips BX, et al. Real-time ophthalmoscopic findings of superselective intraophthalmic artery chemotherapy in a nonhuman primate model. Arch Ophthalmol. 2011;129(11):1458-1465.

284. Yang X, Iyer AK, Singh A, et al. Cluster of Differentiation 44 Targeted Hyaluronic Acid Based Nanoparticles for MDR1 siRNA Delivery to Overcome Drug Resistance in Ovarian Cancer. Pharmaceutical research. 2015;32(6):20972109. 


\section{APPENDIX. TABLES OF PRIMERS AND ANTIBODIES USED}

Table A-1. List of all MRNA primers used.

\begin{tabular}{|c|c|c|}
\hline Gene & Manufacturer & Identifier \\
\hline$M M P 2$ & Applied Biosystems, Thermo Scientific & Hs01548727_m1 \\
\hline$M M P 7$ & Applied Biosystems, Thermo Scientific & Hs01042796_m1 \\
\hline$M M P 9$ & Applied Biosystems, Thermo Scientific & Hs00234579_m1 \\
\hline MMP14 & Applied Biosystems, Thermo Scientific & Hs01037003_g1 \\
\hline PDGFRA & Applied Biosystems, Thermo Scientific & Hs_00998018_m1 \\
\hline PDGFRB & Applied Biosystems, Thermo Scientific & Hs01019589_m1 \\
\hline$P D G F A$ & Applied Biosystems, Thermo Scientific & Hs00234994_m1 \\
\hline$P D G F B$ & Applied Biosystems, Thermo Scientific & Hs_00966522_m1 \\
\hline$M D M 2$ & Applied Biosystems, Thermo Scientific & Hs00540450_m1 \\
\hline MDM4 & Applied Biosystems, Thermo Scientific & Hs00910358_m1 \\
\hline$V E G F A$ & Applied Biosystems, Thermo Scientific & Hs00900055_m1 \\
\hline FLT1 & Applied Biosystems, Thermo Scientific & Hs01052961_m1 \\
\hline HPRT1 & Applied Biosystems, Thermo Scientific & Hs02800695_m1 \\
\hline$A B C B 1$ & Applied Biosystems, Thermo Scientific & Hs00184500_m1 \\
\hline$A B C C 1$ & Applied Biosystems, Thermo Scientific & Hs01561483_m1 \\
\hline$A B C C 2$ & Applied Biosystems, Thermo Scientific & Hs00960489_m1 \\
\hline$A B C G 2$ & Applied Biosystems, Thermo Scientific & Hs01053790_m1 \\
\hline$C D 44$ & Applied Biosystems, Thermo Scientific & Hs01075864_m1 \\
\hline TaqMan $^{\circledR}$ PreAmp Master Mix & Applied Biosystems, Thermo Scientific & Cat\# 4391128 \\
\hline $\operatorname{TaqMan}^{\circledR}$ Universal Master Mix & Applied Biosystems, Thermo Scientific & Cat\# 4440040 \\
\hline SuperScript ${ }^{\circledR}$ VILO cDNA & Invitrogen, Thermo Scientific & Cat\# 11754250 \\
\hline Synthesis Kit & & \\
\hline
\end{tabular}


Table A-1. List of all antibodies used.

\begin{tabular}{|c|c|c|}
\hline Antibody & Manufacturer & Identifier \\
\hline Anti-human PDGFR $\beta$ & Abcam & Cat\# 16868 \\
\hline CFSE Cell Division Tracker Kit & BioLegend & Cat\# 423801 \\
\hline DRAQ $^{\mathrm{TM}}$ & BioLegend & Cat\# 424101 \\
\hline Monoclonal mouse anti-VEGF-A & BioLegend & Cat\# 16701 \\
\hline Monoclonal mouse anti-CD140b APC & BioLegend & Cat\# 323608 \\
\hline anti-human CD44 PerCP/Cy5.5 & BioLegend & Cat\# 103032 \\
\hline eFFLUX-ID ${ }^{\circledR}$ Green multidrug resistance dye & ENZO Lifesciences & ENZ-51029-K100 \\
\hline Monoclonal rabbit anti-MMP2 & Cell Signaling Technology & Cat\# 13132 \\
\hline Polyclonal rabbit anti-MMP9 & Cell Signaling Technology & Cat\# 3852 \\
\hline Polyclonal rabbit anti-E2F & Cell Signaling Technology & Cat\# 3742 \\
\hline Monoclonal $\beta$-actin HRP-conjugated & Cell Signaling Technology & Cat\# 12620 \\
\hline Polyclonal rabbit anti-AKT & Cell Signaling Technology & Cat\# 9272 \\
\hline Monoclonal rabbit anti-pAKT & Cell Signaling Technology & Cat\# 4058 \\
\hline Monoclonal rabbit anti-BCL2 & Cell Signaling Technology & Cat\# 2870 \\
\hline Monoclonal rabbit anti-GAPDH & Cell Signaling Technology & Cat\# 8884 \\
\hline Anti-mouse IgG HRP-linked Antibody & Cell Signaling Technology & Cat\# 7076 \\
\hline Anti-rabbit IgG HRP-linked Antibody & Cell Signaling Technology & Cat\# 7074 \\
\hline Monoclonal rabbit anti-p65 & Cell Signaling Technology & Cat\# 8242 \\
\hline Anti-human phosphorylated PDGFR $\beta$ & Santa Cruz & Cat\# sc-339 \\
\hline Polyclonal rabbit anti-PDGFR $\beta$ & Santa Cruz & Cat\# sc-432 \\
\hline Monoclonal mouse anti-pPDGFR $\beta$ & Santa Cruz & Cat\# sc-373805 \\
\hline Monoclonal mouse anti-VEGF & Santa Cruz & Cat\# sc-53462 \\
\hline Monoclonal mouse anti-Flk-1 & Santa Cruz & Cat\# sc-6251 \\
\hline Monoclonal mouse anti-p-Flk-1 & Santa Cruz & Cat\# sc-101821 \\
\hline Monoclonal mouse anti-MDM2 & Santa Cruz & Cat\# sc-965 \\
\hline Monoclonal mouse anti-pMDM2 & Santa Cruz & Cat\# sc-53368 \\
\hline Donkey anti-rabbit Alexa Fluor 647 & Thermo Scientific & Cat\# A31573 \\
\hline Goat anti-mouse Alexa Fluor 700 & Thermo Scientific & Cat\# A21036 \\
\hline Donkey anti-rabbit Alexa Fluor 488 & Thermo Scientific & Cat\# A21206 \\
\hline
\end{tabular}




\section{VITA}

Zachary Keith Goldsmith was born 1992 and was adopted by his parents, Noel Keith and Pamela Jean Goldsmith. He lived in Germantown, Tennessee and attended Christian Brothers High School, graduating in 2010. He attended Kansas State University in Manhattan, Kansas and earned his Bachelor of Science degree in Microbiology in 2014. He was then accepted into the Biomedical Sciences PhD program within the College of Graduate Health Sciences at the University of Tennessee Health Science Center in Memphis, Tennessee in 2014. He anticipates completion of the requirements for his Doctor of Philosophy in 2018. 